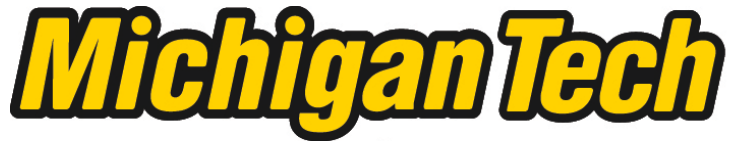 \\ Michigan Technological University Create the Future Digital Commons @ Michigan Tech
}

Dissertations, Master's Theses and Master's Reports - Open

Dissertations, Master's Theses and Master's

Reports

2011

Nanomechanics of cellulose crystals and cellulose-based polymer composites

Anahita Pakzad

Michigan Technological University

Follow this and additional works at: https://digitalcommons.mtu.edu/etds

Part of the Mechanical Engineering Commons

Copyright 2011 Anahita Pakzad

\section{Recommended Citation}

Pakzad, Anahita, "Nanomechanics of cellulose crystals and cellulose-based polymer composites", Dissertation, Michigan Technological University, 2011.

https://doi.org/10.37099/mtu.dc.etds/393

Follow this and additional works at: https://digitalcommons.mtu.edu/etds

Part of the Mechanical Engineering Commons 


\title{
NANOMECHANICS OF CELLULOSE CRYSTALS AND CELLULOSE-BASED POLYMER COMPOSITES
}

\author{
by \\ Anahita Pakzad

\begin{abstract}
A DISSERTATION
Submitted in partial fulfillment of the requirements for the degree of DOCTOR OF PHILOSOPHY

(Mechanical Engineering-Engineering Mechanics)
\end{abstract} \\ MICHIGAN TECHNOLOGICAL UNIVERSITY
}

2011

(C) 2011 Anahita Pakzad 
This dissertation, "Nanomechanics of Cellulose Crystals and Cellulose-Based Polymer Composites," is hereby approved in partial fulfillment of the requirements for the Degree of DOCTOR OF PHILOSOPHY IN MECHANICAL ENGINEERING-ENGINEERING MECHANICS.

Department of Mechanical Engineering-Engineering Mechanics

Signatures:

Dissertation Advisor

Reza S. Yassar

Committee Member

Patricia A. Heiden

Committee Member

Gregory M. Odegard

Committee Member

Dennis DeSheng Meng

Department Chair

William W. Predebon

Date 
To my parents 


\section{Table of Contents}

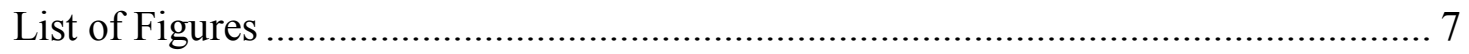

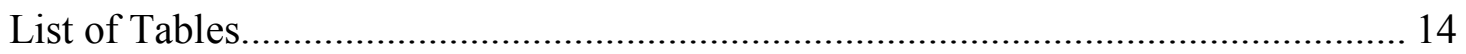

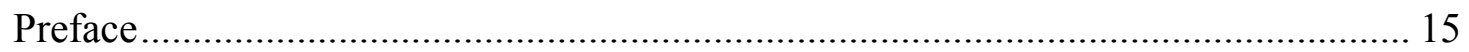

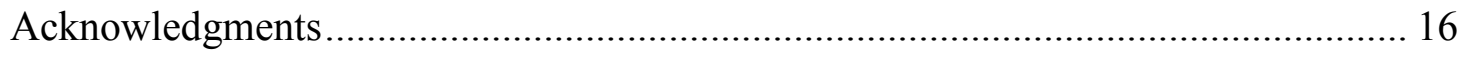

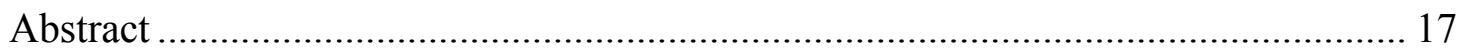

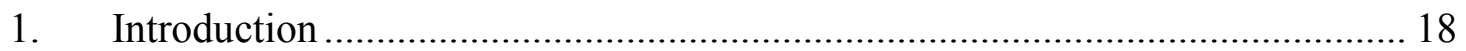

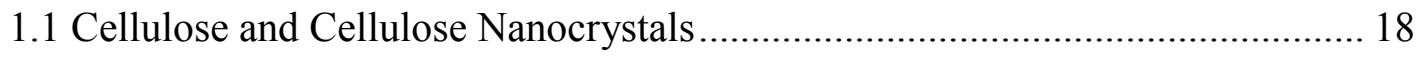

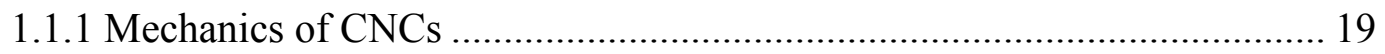

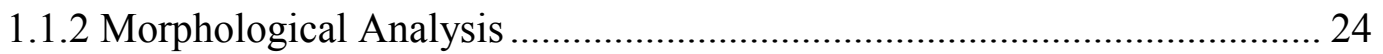

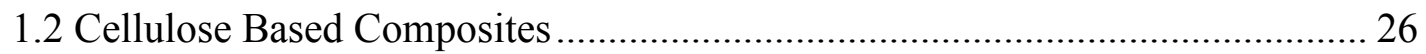

1.2.1 Mechanics of CNC Composites ............................................................ 27

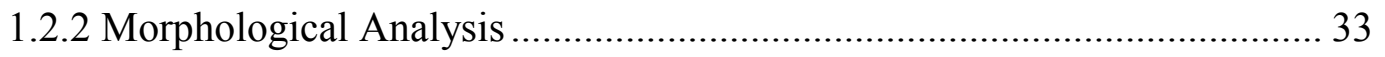

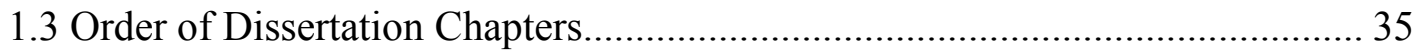

2. Preparation of Cellulose Nanocrystals ............................................................ 37

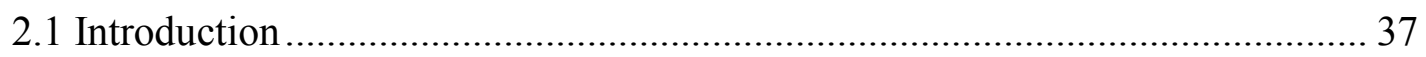

2.2 CNC Preparation and Characterization ......................................................... 37

2.2.1 Effect of CNC Source on Diameter and Aspect Ratio ................................. 38

2.2.2 Effect of Preparation Steps on CNC Size ................................................... 40

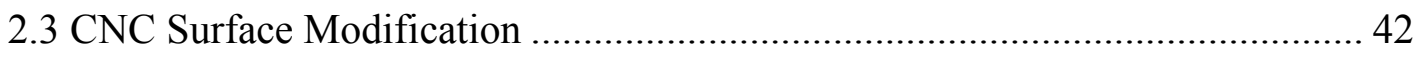

2.3.1 Preparation of Carboxylated Cotton CNCs................................................ 43

3. Revealing the 3D Internal Structure of Natural Polymer Microcomposites Using X-ray Ultra Microtomography ..................................................................................... 44

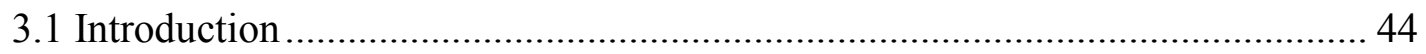

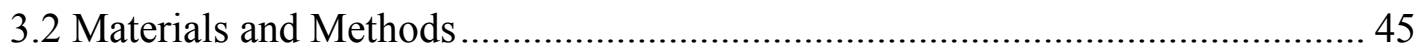

3.3 Selection of Target Material and 3D Reconstruction.......................................... 49

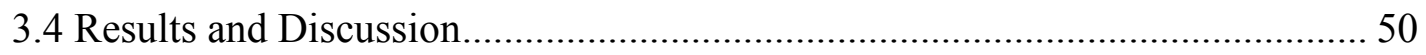

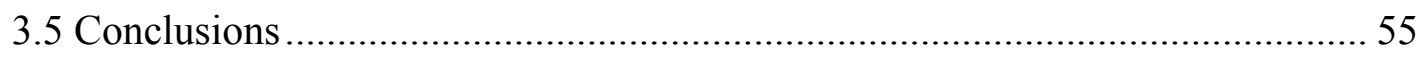

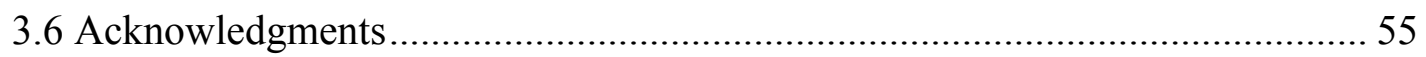

4. Elastic Properties of Thin Poly(vinyl alcohol)-Cellulose Nanocrystal Membranes. 56 


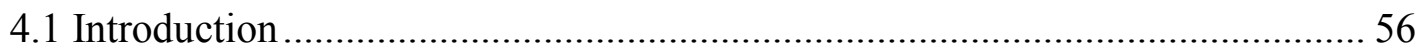

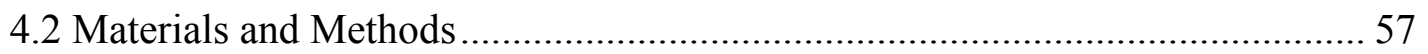

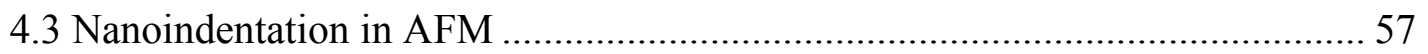

4.4 Calculation of Nanomechanical Properties ......................................................... 58

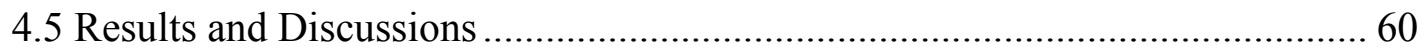

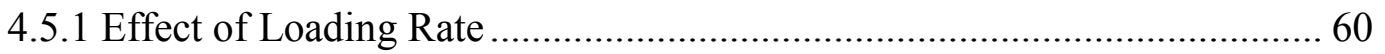

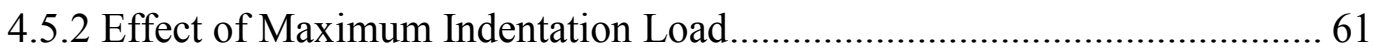

4.6 Nanomechanical Properties of PVA-PAA-CNC Membranes ............................. 62

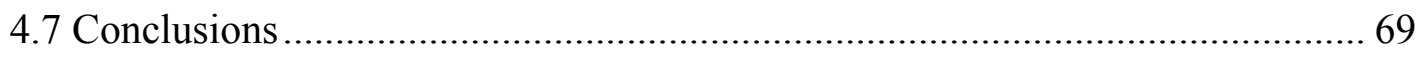

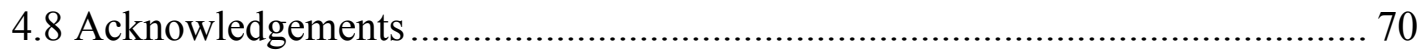

5. Gradient of Nanomechanical Properties in the Interphase of Cellulose

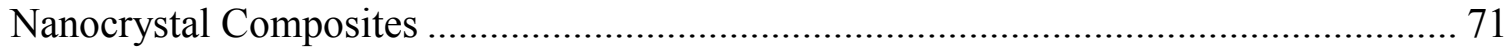

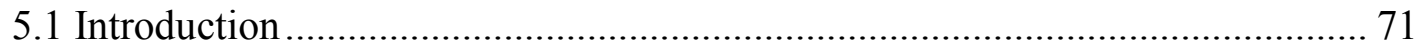

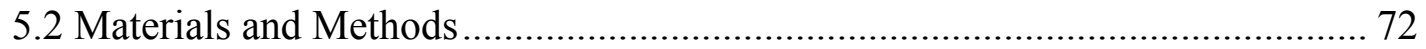

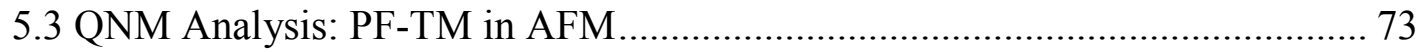

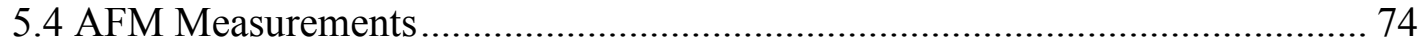

5.5 Elastic Modulus and Adhesion at the Interphase ……………………............. 75

5.6 Interphase Thickness and CNC Diameter...................................................... 79

6. Size Effects on the Nanomechanical Properties of Cellulose I Nanocrystals .... 82

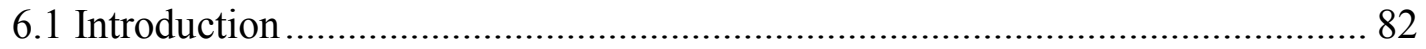

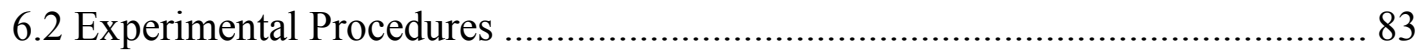

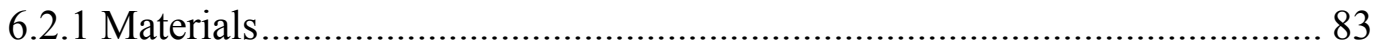

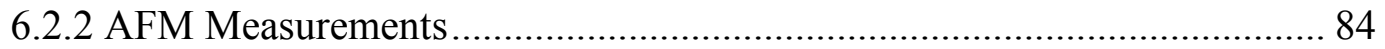

6.2.3 Calculation of CNC Transverse Elastic Modulus ....................................... 85

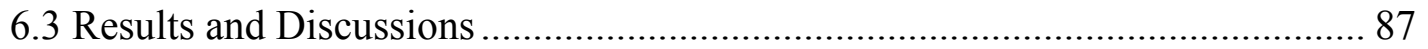

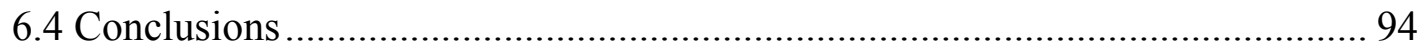

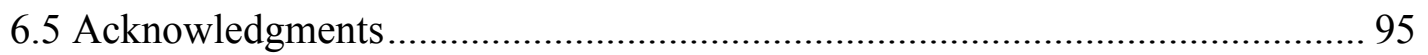

7. Effect of Surface chemistry on Mechanics of Cellulose Nanocrystals .............. 96

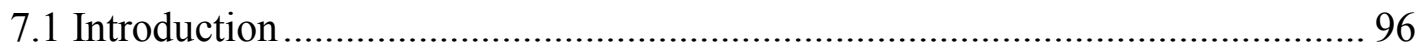

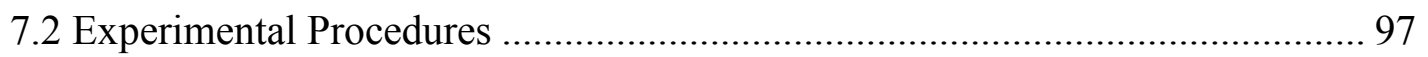

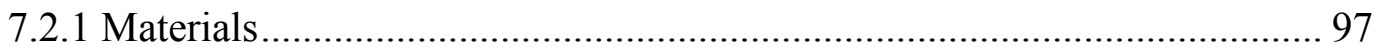

7.2.2 Atomic Force Microscopy..................................................................... 98

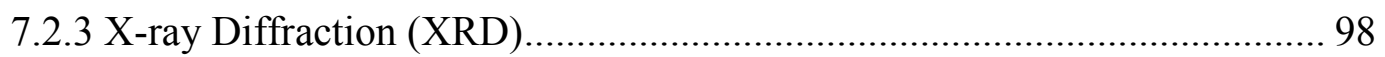

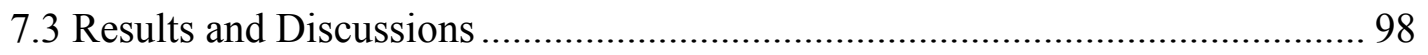




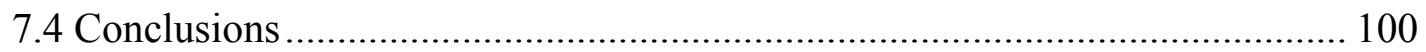

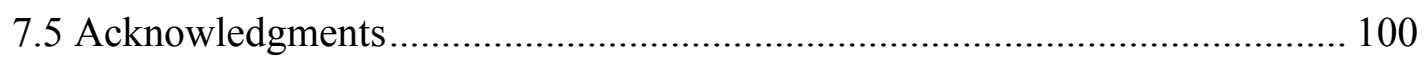

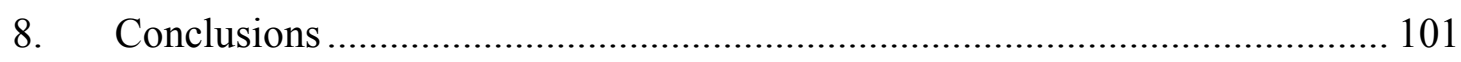

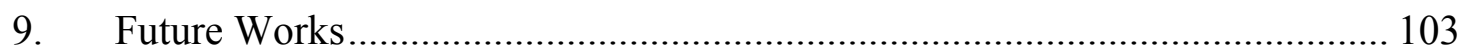

9.1 3D Distribution of CNCs in Polymer Composites........................................... 103

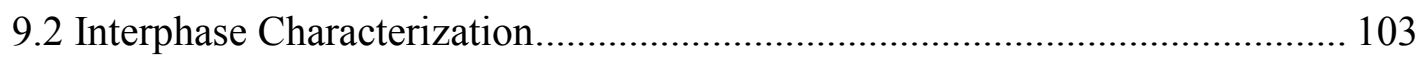

9.3 CNC and CNC Composites for Biomedical Applications ............................... 104

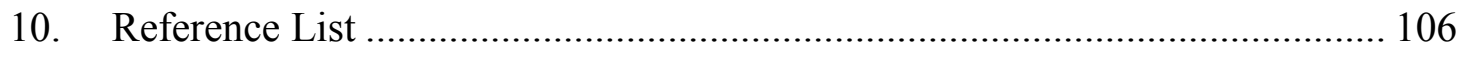

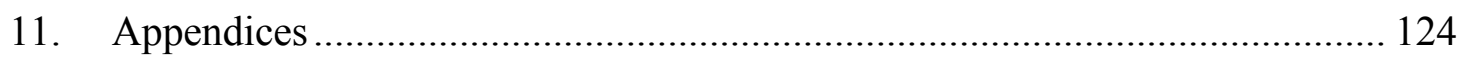

Appendix A: Atomic Force Microscopy ........................................................ 124

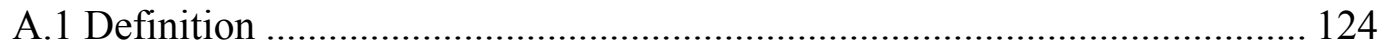

A.2 Scientific Fundementals ....................................................................... 125

A.3 Mechanical Characterization of Surfaces in AFM ..................................... 125

A.4 Key Applications in Surface Mechanics .................................................... 128

A.5 Electrical Characteriztion in AFM .......................................................... 129

A.6 Key Applications of Electrical AFM ....................................................... 134

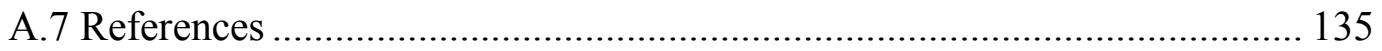

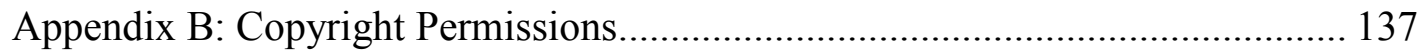

B.1 Permission for Figures Used in Chapter 1 ................................................. 137

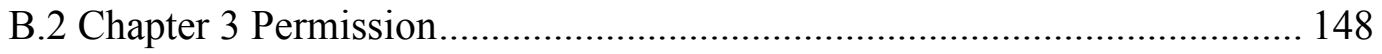

B.3 Chapter 4 Permission............................................................................ 153

B.4 Chapter 5 Permission............................................................................. 155

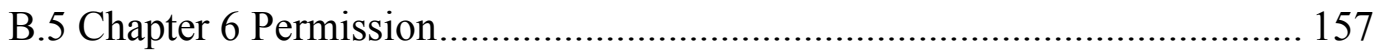

B.6 Appendix A and Chapter 1 Permissions...................................................... 160

B.7 Permission for Figure 11.1 ...................................................................... 162 


\section{List of Figures}

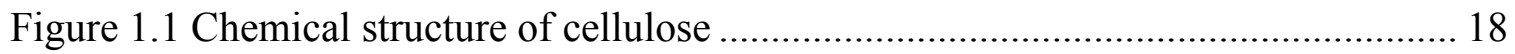

Figure 1.2 Schematic presentation of crystalline structure of cellulose I, crystalline parts are connected by amorphous areas...................................................................... 19

Figure 1.3 Clamped fiber with length equal to (L). Force is applied to the fiber with distance (a) from the edge using the AFM cantilever tip............................................ 22

Figure 1.4 Intermolecular (blue) and intramolecular (red) hydrogen bonds in cellulose I (a) and cellulose II (b)

Figure 1.5 A SEM image of bacterial CNC pellicles (With kind permission from Springer Science+Business Media: Applied Physics, Bacterial Cellulose: The ultimate nanoscalar cellulose morphology for the production of high strength composites, 80 , 2005, 93-97, Nakagaito). 25

Figure 1.6 A TEM image of tunicin CNC (Reprinted with permission from (ElazzouziHafraoui et al. 2008). Copyright 2008 American Chemical Society)......................... 25

Figure 1.7 AFM image of cotton CNCs (Reprinted with permission from (ElazzouziHafraoui et al. 2008). Copyright 2008 American Chemical Society)......................... 26

Figure 1.8 Effect of $\mathrm{CNC}$ origin on the Young's modulus of $\mathrm{CNC} / \mathrm{starch}$ composites (Orts et al. $2005 \mathrm{Lu}$ et al. 2006; Kvien et al. 2007).................................................. 28

Figure 1.9 Change in elastic modulus and tensile strength (a) and elongation at break (b) of soy protein isolate (SPI) and polyvinyl alcohol (PVA) filled with different amounts of CNC derived from cotton linter (Wang et al. 2006; Roohani et al. 2008)............... 30

Figure 1.10 Change in elastic modulus of soy protein isolate (SPI) and Starch filled with $\mathrm{CNC}$ at various relative humidity (RH) (Wang et al. 2006; Dufresne et al. 2000)...... 31

Figure 1.11 Typical load-displacement curve............................................................... 32

Figure 1.12 Optical Microscopy images of CNC-PVA composites (a) reinforcement without coating; (b) reinforcement coated with ethylene-acrylic oligomer. The dispersion is better in $b$, where less aggregates and filler with smaller diameters are observed (Reprinted from Composites Science and Technology, 67, Wang and Sain, Isolation of naofibers from soybean source and their reinforcing capability on synthetic polymers, 2521-2527, Copyright 2007, with permission from Elsivier)...... 34

Figure 1.13 SEM images of the polyurethane filled with different wt. \% of CNC: a) $0 \mathrm{~b}$ ) 20 c) 30. In contrast with the fracture surface in (a) which is featureless, the fracture surfaces are rough in (b) and (c) and the white dots on them are the CNCs (Reprinted with permission from (Cao et al. 2007). Copyright 2007 American Chemical Society) 
Figure 1.14 TEM images of CNC-PLA composite (Reprinted with permission from (Kvien et al. 2005). Copyright 2005 American Chemical Society)

Figure 2.1 Schematic procedure for acid hydrolysis of commercially available microcrystalline cellulose. After the acid $\left(\mathrm{ex} . \mathrm{H}_{2} \mathrm{SO}_{4}\right)$ is added slowly to the water solution, it should be heated to certain temperatures and kept for certain times. Afterwards, using centrifuge, dialysis against distilled water, sonication and maybe ultra filtering $\mathrm{CNCs}$ are separated. 38

Figure 2.2 Importance of three variables were studied on the resultant size of cottonCNCs. This included shelf life (at two different temperatures), sonication (various times), and fractionation using ultracentrifugation. 38

Figure 2.3 Section analysis on the height image to estimate the diameter of CNC. (a) a 2 $\times 2 \mu \mathrm{m}^{2}$ topography image of filter paper-CNCs. (b) Higher magnification image of the region shown in red box in (a). A line is drawn perpendicular to the axis of the crystal. The topography profile along this line is shown in (c). The vertical distance between the substrate and the highest point on $\mathrm{CNC}$ is equal to the diameter. 39

Figure 2.4 The $5 \times 5 \mu^{2}$ topography images of (a) Filter paper, (b) Cotton batting, and (c) MCC CNCs. 40

Figure 2.5 Centrifuge vials (a) before and (b) after the ultracentrifugation process, where a small amount of solid is seen at the bottom of the vial. 42

Figure 2.6 The $5 \times 5 \mu \mathrm{m}^{2}$ topography images of cotton CNCs (a) before (b) and (c) after ultracentrifugation. (b) shows the solid and (c) shows the turbid portions, after ultracentrifugation. 42

Figure 3.1 (a) Main components of the XuM inside SEM, in order to generate X-rays, a metal target is positioned under the electron beam using the target module. X-rays pass through the sample and create the 2D image on the CCD camera. (b) Point projection geometry in XuM; the magnification $\left(M=R_{2} / R_{l}\right)$ can be changed by moving the sample towards or away from the X-ray source. 48

Figure 3.2 Relative intensity and resolution of XuM metal targets. The diameter of the circles on the graph represents the imaging resolution in micrometers. Intensities were reported relative to intensity of $\mathrm{W}$ at $30 \mathrm{KeV}$.

Figure 3.3 2D XuM images of PCL samples obtained under (a) Ag, (b) V, (c) W, (d) Au metal targets. The accelerating voltage was fixed at $30 \mathrm{KeV}$. The choice of $\mathrm{V}$ target was preferred because of its optimal contrast and flux. 50

Figure 3.4 Engineering stress-strain curves for PCL composites: black: PCL 0, red: PCL 10, green: PCL 30, and purple: PCL 50. 51 
Figure 3.5 SEM images from the fracture surface of CMC-PCL composites: a) PCL 0, b) PCL 10, c) PCL 30, d) PCL 50; Arrows in (c) show voids that were created as a result of CMC being pulled out during the tensile test. (Scale bars are $100 \mu \mathrm{m}$ ) 51

Figure 3.6 2D XuM images of PCL composites: (a) PCL 0, (b) PCL 10, (c) PCL 30, and (d) PCL 50. Some of the CMCs are shown by black arrows on the images. The yellow arrow in (a) shows a crack in the sample, which was created during sample preparation (phase contrast). (Scale bars are $200 \mu \mathrm{m}$ ). 52

Figure 3.7 3D reconstruction of a PCL sample filled with $10 w t \% \mathrm{CMC}$. CMC can be recognized as bright regions. (a) Horizontal slice of 340 by $543 \mu^{2}$ through the sample. (b) Vertical plane through the specimen. (c) 3D volume is shown along with two slices through the sample. PCL and CMC are shown as gray and green zones.... 53

Figure 3.8 3D visualization of PCL samples filled with a) 0 b) 10 c) 30 and d) $50 w t . \%$ CMCs. PCL and CMCs are shown in gray and green respectively. 53

Figure 3.9 Histograms show the distribution of the CMC-cluster diameters in PCL composites: a) PCL 10 b) PCL 30 c) PCL 50. As opposed to PCL 10 and PCL 30, the number of small sized $(<5$ micrometers) clusters is higher in PCL 50. 54

Figure 4.1 (a) Effect of loading rate on the elastic recovery parameter ( $\xi$ ). (b) Variation of modulus as a function of loading rate. The error bars show the standard deviations. Loading rate $>0.4 \mathrm{~Hz}$ results in higher $\xi$ and consistency in elastic property measurements. The red dashed line shows the optimal value after which loading rate is high enough to eliminate most of plastic and viscoelastic deformations. 61

Figure 4.2 (a) Effect of maximum applied load on the resultant modulus values. (b) Corresponding indentation imprints shown by numbers in (a). At indentation loads lower than $11 \mu N$, elastic modulus varied with the applied load. On the other hand, when applied loads are greater than $11 \mu N$, consistent results were obtained. 62

Figure 4.3 AFM topography images of $10 \times 10 \mu \mathrm{m}^{2}$ area: (Top) before and (bottom) after nanoindentation. (a ,f) PVA 100-PAA 0-CNC 0; (b, g) PVA 90-PAA10-CNC 0; (c, h) PVA 85-PAA 0-CNC 15; (d, i) PVA 80-PAA 10-CNC 10; and (e, j) PVA 70-PAA 10CNC 20. CNCs are pointed with yellow arrows in (c), (d) and (e). Average roughness values $(\mathrm{Ra})$ are shown on height images before nanoindentation. Areas in yellow squares in (f) and (i) are shown in 3D in Figure 4.4. 63

Figure 4.4 High magnification three dimensional AFM topography images of nanoindentation imprints at $1 \times 1 \mu \mathrm{m}^{2}$ areas shown with yellow squares in Figure 4.3. (a) PVA 100-PAA 0-CNC 0 with no significant pile-up and (b) PVA 80-PAA 10-CNC 10 with pile-up around the indentation imprint.

Figure 4.5 Representative indentation curves on pure PVA and four nanocomposite films with different $\mathrm{CNC}$ and PAA content. The difference in the slope of the curves shows the difference in the elastic moduli. Pop-in and kick-back points on the curves occur as a result of sudden energy releases during loading and unloading, respectively. 64 
Figure 4.6 Representative indentation curves on PVA 80-PAA 10-CNC 10 at two different locations. The blue curve represents the indentation on a $\mathrm{CNC}$, while the indentation of the polymer matrix is shown by the black curve. The indentation locations are shown on the topography image by the arrows. There is a small difference between these two curves in terms of maximum penetration under the applied load, slope and adhesion, but these differences are not substantial. 65

Figure 4.7 Comparison of the elastic modulus of nanocomposite films (PVA 100-PAA 0CNC 0, PVA 90-PAA 10-CNC 0, PVA 85-PAA 0-CNC 15, PVA 80-PAA 10-CNC 10, PVA 70-PAA 10-CNC 20) obtained from tensile test (red) and nanoindentation by fitting the Hertz model to loading (light blue) and unloading (dark blue) curves. Nanoindentation results from fitting the Hertz model on the loading curve resulted in values closer to bulk mechanical properties.

Figure 4.8 Indentation/tensile elastic modulus ratio in different PVA nanocomposites. Nanoindentation/tensile modulus ratio was larger than 1 for all samples. In the nanoindentation method, the elastic modulus was calculated by fitting the Hertz model to the loading curves. 67

Figure 4.9 Topography images from two different location $(a, b)$ on the PVA 85-PAA 0CNC 15 sample, show high degree of inhomogeneity in the sample. Scan area is $5 \times 5 \mu \mathrm{m}^{2}$. Yellow arrows show CNCs embedded in PVOH.

Figure 5.1 Left: Schematic of force-separation curve collected at each pixel. The blue curve (trace) represents the loading portion and the red curve (retrace) indicates the unloading part. Part of the unloading curve (bold dashed line) is used to calculate the Derjaguin-Muller-Toropov (DMT) elastic modulus (Derjaguin et al. 1975). The minimum force in the retrace curve is used for mapping the adhesion properties. Also, the peak force value is used for peak force tapping imaging in AFM. Right: tip-sample interactions during each tap. At (A) there is little or no force on the tip. As the piezo expands (separation decreases), the tip is pulled down (B) by attractive forces such as van de Waals, capillary or electrostatic, until it touches the surface (separation $=0$ ). Then the tip is pushed into the sample until the set point for the peak force is reached (C). The piezo then starts to pull up the tip and the force keeps decreasing (D) to a minimum value, where the tip comes off the surface. After this point forces affecting the tip keep decreasing as separation increases (E) and piezo returns to its initial position. 73

Figure 5.2 (a) Height and (b) adhesion maps of a $50 \times 50 \mu \mathrm{m}^{2}$ region on a PVA 80 - CNC 10- PAA 10 sample. The arrows point to CNCs embedded in the polymer matrix. The presence of $\mathrm{CNCs}$ is easily distinguishable from the adhesion image. 75

Figure 5.3 3.5 $\times 3.5 \mu^{2}$ (a) 3D height, (b) adhesion, (c) elastic modulus maps. Inset in (b) shows the scanning area (PVA 80- CNC 10- PAA 10). (d) The average adhesion and elastic modulus profiles of the area in the red boxes in (b) and (c). Polymer matrix and $\mathrm{CNC}$ regions are marked on the profile. Interphase region is distance that the overlapping in adhesion and modulus profiles is observed. 76 
Figure 5.4 Structure models of PVA membranes containing (a) no PAA, (b) PAA and before cross-linking, and (c) PAA and after cross linking. In PVA-CNC membrane, only hydrogen bonds between CNC and PVA exist at the interphase. In the PVACNC-PAA case (before cross-linking), PVA molecules form hydrogen bonds with hydroxyl groups on the CNC surface. Some PAA molecules also exist in the vicinity of CNC. After cross-linking, ester bonds form and the population of these bonds decreases as the distance from CNC surface increases. (Figure is not to scale) .......... 78

Figure 5.5 Average adhesion profiles (arbitrary unit, a.u.) in PVA 80- CNC 10- PAA 0 (square) and PVA 85- CNC 15- PAA 0 (triangle) composites. Dashed lines were used to measure the rate of change in adhesion at the interphase (nN.nm ${ }^{-1}$ ). 78

Figure $5.61 \times 1 \mu \mathrm{m}^{2}$ height (a) and adhesion (b) maps on a single CNC. (c) Average adhesion profile of the area shown by red rectangle in adhesion map in (b). Using this profile, CNC diameter $(9 \mathrm{~nm})$ and average thickness of the interphase were measured. 79

Figure 5.7 Change in interphase thickness with CNC diameter. Red circles show average interphase thickness of the CNCs. The trend line (dashed gray line) shows an increasing trend in the interphase thickness with the $\mathrm{CNC}$ diameter..... 80

Figure 6.1 3D quarter symmetry model in FEA; (a) shows the elements and finer mesh at the contact regions at CNC-AFM tip and CNC-Mica interfaces, and (b) shows the deformation along the vertical axis at maximum indentation. The color contour in (b) represents the amount of deformation in the model, which varied from $-2 \mathrm{~nm}$ (red) to $1.1 \mathrm{~nm}$ (dark blue). 86

Figure $6.2 \mathrm{~F}$ - $h$ curves on a wood-CNC with diameter equal to $3.1 \mathrm{~nm}$ (black diamonds) and cotton-CNC with diameter equal to $6.4 \mathrm{~nm}$ (red squares) along with fit curves with varying elastic moduli obtained from FEA. 87

Figure $6.31 \times 1 \mu m^{2}(a, c)$ topography and (b, d) adhesion maps of (a, b) wood and (c, d) cotton CNCs. The topography (e) and adhesion (f) profiles were obtained along the lines shown on AFM maps. Dotted red lines and solid green lines correspond to cotton- and wood-CNCs, respectively. Yellow crosses show the direction of the profile lines. 88

Figure 6.4 Change in $E_{t}$ (Calculated using FEA) with the CNC diameter (a) cotton-CNCs (red circles and black crosses show the experimental data and the fitted curve, respectively) and (b) wood-CNCs (black diamonds and black crosses show the experimental data and the fitted curve, respectively). The fitted curves clearly show that as the $\mathrm{CNC}$ diameter decreases, $E_{t}$ increases. $m$ and $\delta$ resulted from fitting are shown on the graphs. 91

Figure 6.5 (a) Schematic of crystal structure of cellulose along the fiber. Light and dark green correspond to crystalline and amorphous areas in cellulose and orange represents the polycrystalline surface layer. Structural defects are shown by small yellow rectangles. During acid hydrolysis, amorphous regions are removed and 
cellulose nanocrystals are extracted. (b) Change in cross section of a CNC as the hydrolysis exposure increases. At longer reaction time, acid protons attack the polycrystalline surface and crystalline core of $\mathrm{CNC}$ and resultant crystals will have smaller diameter and fewer defects in their structure. 94

Figure 7.1 Two common syntheses of CNCs that result in different surface properties: suldonation brings sulfate esters (top) and carboxylation results in carboxylic groups (bottom) on the CNC surface. 97

Figure 7.2 Change in $E_{t}$ (Calculated using FEA) with the CNC diameter. Diamonds and squares are the experimental data, and the crosses show the fitted curves, for carboxylated- and sulfated- CNCs. The fitted curves clearly show that as the CNC diameter decreases, $E_{t}$ increases. $m$ resulted from fitting is shown next to the curves.98

Figure 7.3 Powder diffraction patterns for sulfated (dashed red line) and carboxylated (dotted black line) CNCs. Compared to S-CNC, C-CNC had larger crystalline peaks. 99

Figure 11.1 Schematic representation of an AFM. The cantilever-tip system is deflected by the surface topography of the sample. Cantilever deflections are detected with a laser-optical set-up. The four-segment photodiode detects normal forces (normal force microscopy (NFM)) and frictional forces (FFM) affecting the tip. In most AFM systems the sample rests on a piezotube scanner (not shown in this figure) which allows a scanning motion in $x$ - and $y$-directions as well as movement in $z$-direction (Reprinted from Surface Science, 491 (3), KD Jandt, Atomic force microscopy of biomaterilas surfaces and interfaces, 303-332, 2001 with permission from Elsivier) . 124

Figure 11.2 Interatomic force variation versus the distance between AFM tip and sample. 125

Figure 11.3 Schematic of a common Left: cantilever deflection versus piezo height and Right: corresponding cantilever deflection versus distance. 126

Figure 11.4 Schematics of Hertz, JKR and DMT models for the surface forces at the contact regime (rigid tip and compliant substrate). 127

Figure 11.5 The height image and adhesion maps of a cellulose nanocomposite are shown. The nanocrystals are embeded in the polymer matrix and are not detectable in the height image. However, the adhesion map obtained by peak force AFM mode detects the presence of nanocrystals marked by arrows. The nanocrystals have lower adhesion force to the AFM canteliver in compasirson to the polymer matrix. The adhesion map is quantified for values up to $25 n N$...... 129

Figure 11.6 A schematic of electric force microscopy (EFM) is shown. A variable and a constant voltage source are connected in series between the tip and the sample. Deflection of the cantilever will be measured by laser beam and a position sensitive 
detector (PSD). Electric field due to the presence of charges, $q$, can be detected by change in the amplitude or oscillation phase of the cantilever. 130

Figure 11.7 A schematic of scanning capacitance microscope (SCM) is shown. Type of the dopant can be determined by the sign of $(d C / d V)$, which is positive for n-typed dopant and negative for $\mathrm{p}$-typed dopant. Dopant concentration is inversely related to $d C / d V$, i.e. higher concentration results in smaller $d C / d V$, and vice versa. .............. 131

Figure 11.8 (Top) Schematic diagram of the PFM is shown. There is an alternative voltage source (Vac cos $\omega \mathrm{t}$ ) between the conductive AFM tip and conductive substrate. Deflection of the cantilever will be monitored by position sensitive photo detector (PSPD). The feedback control on z-height gives the topography image while the lock-in amplifier provides piezo-response image. (Down) Phase image of piezorsponse of a $\mathrm{ZnO}$ nanobelt shows domains along the axis of nanobelt............ 133 


\section{List of Tables}

Table 1.1 Elastic modulus of crystalline regions of cellulose polymorphs obtained by Xray diffraction (Nishino et al. 1995) ......................................................................... 20

Table 1.2 Elastic modulus of $\mathrm{CNCs}$ with different origins, gained using Raman

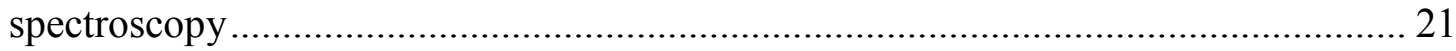

Table 1.3 Elastic modulus of CNCs with different origins, gained using atomic force

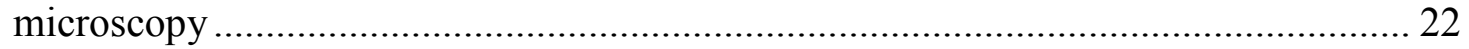

Table 2.1 Comparison of CNCs from different origins ................................................ 40

Table 2.2 Effect of sonication time on CNC length measured by DLS............................ 41

Table 2.3 Effect of ultracentrifugation on CNC length measured by DLS. .................... 42

Table 3.1 The UTS values in PCL microcomposites ......................................................... 46

Table 3.2 Statistical parameters of CMC-polymer composites ........................................ 54

Table 6.1 Summary of the AFM measurements on individual CNCs ............................ 89

Table 11.1 Hertz, JKR and DMT models for the contact regime. (contact radius $=a$, sample deformation $=\delta$, adhesion force $=F_{a d}$, tip radius $=R$, Adhesion work per unit area $=W$, applied force $=F$, reduced Young's modulus $=\mathrm{E}_{\text {red }}$ ) 128 


\section{Preface}

This dissertation is based upon studies conducted between January 2008 and December 2011 at the Mechanical Engineering-Engineering Mechanics Department, Michigan Technological University, Houghton, Michigan, United States. All the literature review, experimental desings, tests and analysis of the results we performed by myself except for:

- The work presented in Chapter 2 was performed in collaboration with Mr. W. Grant in Dr. Heiden's research group (Chemistry Department, Michigan Tech).

- The samples studies in Chapter 3 were prepared by a student in Dr. Heiden's lab (Chemistry Department, Michigan Tech) and the statistical analyses on XuM data were performed by Mr. N. Parikh.

- Composite samples studied in Chapter 4 and 5 were prepared in Dr. Simonsen's lab (Oregon State University). The tensile test results presented in Chapter 3 were also performed at Oregon State University.

- Cotton CNC samples studied in Chapter 6 and 7 were prepared by students in Dr. Simonsen's research group.

- XRD tests in chapter 7 were performed by Mr. E. Laitila at Materials Science Department at Michigan Tech.

- Sections A.1 to A.4 in Appendix A were written by Dr. R. S. Yassar and Dr. K. Momeni. 


\section{Acknowledgments}

Of the many people I have to thank, it is my advisor, Dr. Reza S. Yassar, whose knowledge, guidance, patience, and support made this research possible and successful.

I would also like to acknowledge my advisory committee members, Dr. P. Heiden, Dr. G. Odegard and Dr. D. Meng, for encouraging me to think outside the box and for their guidance and constructive discussions throughout my research.

Working with our external collaborator, Dr. J. Simonsen at Oregon State University has been a delight. His experience and knowledge helped me find my way at different steps in my research and without the samples prepared in his lab this research would have not been completed.

Additionally, I would like to thank Mr. O. Mills, Mr. M. LaCourt, Mr. E. Laitila, and my friends in Dr. Yassar's research group and Dr. Heiden's research group, who helped me with some of my experiments.

I am and always will be grateful for the love and support of my family. My parents and my brother have always believed in me. They gave me the courage to overcome the obstacles that I faced, from the first day at preschool until I finished my PhD. 


\section{Abstract}

Cellulose-polymer composites have potential applications in aerospace and transportation areas where lightweight materials with high mechanical properties are needed. In addition, these economical and biodegradable composites have been shown to be useful as polymer electrolytes, packaging structures, optoelectronic devices, and medical implants such as wound dressing and bone scaffolds.

In spite of the above mentioned advantages and potential applications, due to the difficulties associated with synthesis and processing techniques, application of cellulose crystals (micro and nano sized) for preparation of new composite systems is limited. Cellulose is hydrophilic and polar as opposed to most of common thermoplastics, which are non-polar. This results in complications in addition of cellulose crystals to polymer matrices, and as a result in achieving sufficient dispersion levels, which directly affects the mechanical properties of the composites.

As in other composite materials, the properties of cellulose-polymer composites depend on the volume fraction and the properties of individual phases (the reinforcement and the polymer matrix), the dispersion quality of the reinforcement through the matrix and the interaction between CNCs themselves and CNC and the matrix (interphase). In order to develop economical cellulose-polymer composites with superior qualities, the properties of individual cellulose crystals, as well as the effect of dispersion of reinforcements and the interphase on the properties of the final composites should be understood.

In this research, the mechanical properties of CNC polymer composites were characterized at the macro and nano scales. A direct correlation was made between:

- Dispersion quality and macro-mechanical properties

- Nanomechanical properties at the surface and tensile properties

- CNC diameter and interphase thickness

Lastly, individual CNCs from different sources were characterized and for the first time size-scale effect on their nanomechanical properties were reported. Then the effect of CNC surface modification on the mechanical properties was studied and correlated to the crystalline structure of these materials. 


\section{Introduction ${ }^{1}$}

\subsection{Cellulose and Cellulose Nanocrystals}

Cellulose is considered as one of the most abundant materials in the nature. It exists in the structure of plants, sea animals and it is also made by some bacteria. In most of these structures cellulose functions as a reinforcing element. Besides being abundant, cellulose has many other qualities that make it attractive for usage in fabrication of composite materials (Moon et al. 2011). A number of these exceptional qualities are: renewable nature, biodegradability, biocompatibility, lower cost in comparison to synthetic fillers, low density, impressive strength to weight capability, easy processability, because of its nonabrasive nature and relatively reactive surface (Azizi Samir et al. 2004-a; Glasser 2004; Azizi Samir et al. 2005; Hamad 2006; Marcovich et al. 2006; Muller et al. 2006; Cao et al. 2007; Noorani et al. 2007).

The chemical structure of cellulose is shown in Figure 1.1. It is a linear homopolymer of $\beta$-D-glucopyranose units which are connected by $(1 \rightarrow 4)$-glycosidic bonds. Cellulose is chiral and because of high density of hydroxyl groups is hydrophilic and can be soluble in water depending on $n$ ( $n$ differs from 500 to 15000).

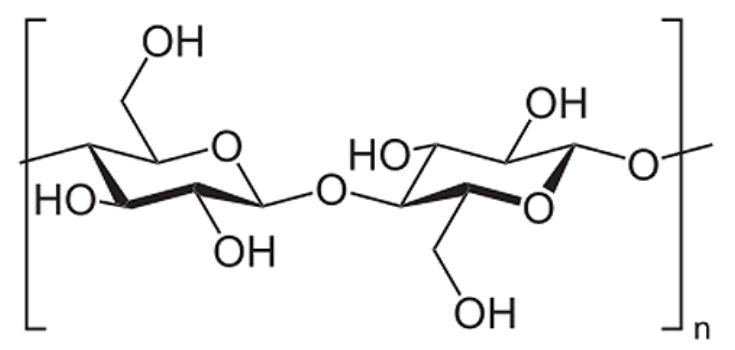

Figure 1.1 Chemical structure of cellulose

The straight nature of cellulose molecule and the existence of hydrogen bonds result in high likeliness of crystallization. Pure cellulose exists in different allomorphs (Zugenmaier 2001; Hamad 2006; Szymanska-Chargot et al. 2011). Cellulose I (natural or native cellulose), consist of two phases: crystalline and amorphous. Amorphous areas connect the crystalline parts as shown in Figure 1.2. The proportion of these two phases depends on the origin of the cellulose and defines the crystallinity degree of the structure.

\footnotetext{
1 The material contained in this chapter was previously published in New Frontiers of Nanocomposite Materials: Novel Principles and Techniques, ed. A. Oechsner and A. Shokuhfar, Springer LLC. See Appendix C for proper documentations.
} 


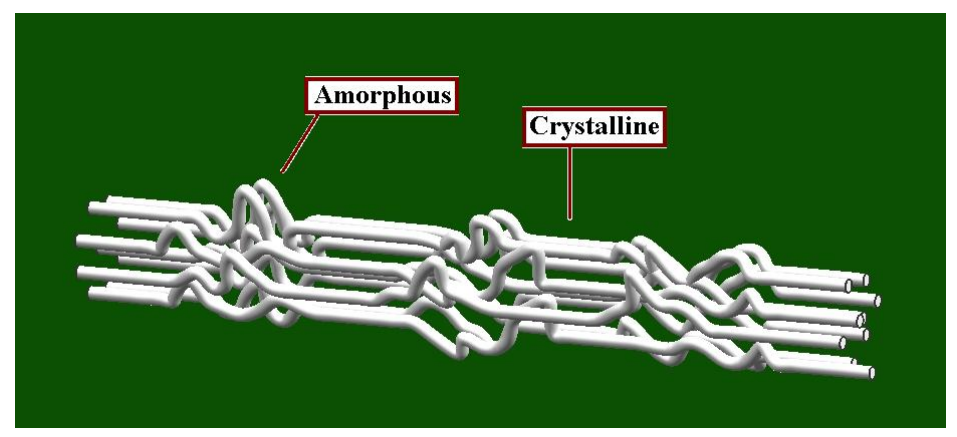

Figure 1.2 Schematic presentation of crystalline structure of cellulose I, crystalline parts are connected by amorphous areas

Cellulose II (regenerated or man-made) is a recrystallized form of cellulose I, and in contrast to cellulose I, has anti parallel strands and inter-sheet cellulose bonding, and it is thermodynamically more stable. Cellulose III can be formed by treating cellulose with liquid ammonia. Depending on what the starting material, cellulose I or II, the ammonia treatment conversion will be denoted as $\mathrm{III}_{1}$ or $\mathrm{III}_{2}$ respectively. Cellulose $\mathrm{III}$ is amorphous. Heat treatment of cellulose III results in cellulose IV, which is also amorphous.

In order for cellulose to be used as nano-reinforcement, the crystalline regions must be separated from the amorphous areas. The most common method for separation of cellulose crystals from amorphous region is acid hydrolysis (See Chapter 2). This includes a chemical treatment for separation of nanocrystals and use of mechanical energy to disperse them in an aqueous suspension. Under suitable conditions (for instance see Bondeson et al. 2006; Tang et al. 2011), acid hydrolysis breaks down the structure of cellulose into individual needle like crystalline rods by disrupting the amorphous regions. This separation happens due to the faster hydrolysis kinematics of amorphous regions than the crystalline parts. The resultant highly crystalline cellulose nanostructures with different aspect ratios ( $\mathrm{L} / \mathrm{D}, \mathrm{L}=$ length, $\mathrm{D}=$ diameter), that depend on the hydrolysis conditions and their crystallinity and origin and can be greater than 200 (BeckCandanedo et al. 2005; Roman and Winter 2006; Elazzouzi-Hafraoui et al. 2008), are referred to as cellulose nanocrystals (CNCs). One should note that CNCs have been entitled, nanorods, nanowires and whiskers as well (Hubbe et al. 2008). All these terms refer to the individual crystalline rods of cellulose, which have at least one dimension less than $100 \mathrm{~nm}$ and an overall length comparable to their diameter.

\subsubsection{Mechanics of CNCs}

The mechanical properties of cellulose-based structures have been investigated from both modeling and experimental prospectives. Early modeling works started in 1930 but were limited to cellulose in general and not nanocrystals in particular. Meyer and Lotmar (1936) were the first who theoretically modeled the mechanical properties of cellulose. They showed that cellulose elastic modulus is related to its chain direction and could be calculated using the chemical bonds force constants of the obtained from vibrational frequencies of the molecules. Their modeling was later modified and extended to other 
synthetic polymer crystals, by Lyons (1959). Treloar (1960) considered the valence angle deformation and further modified these models.

Theoretical work on mechanics of cellulose nanocrystals has received attention only very recently. Tanaka and Iwata (2006) used molecular mechanics simulation and derived values between 124 and $155 \mathrm{GPa}$ for elastic modulus of natural nanocellulose fibers. For this characterization the super cell models with crystal sizes of $1 \times 1 \times 10$ and $4 \times 4 \times 10$ were used, which in comparison to the unit cell method did not require strict symmetries and thus were more suitable for polymers. In order to calculate the elastic modulus, linear relation between the changes in energy density and the half of the square of the compressive or tensile strain were used:

$$
\frac{P-P_{0}}{S * l}=\frac{1}{2} E\left(\frac{l-l_{0}}{l_{0}}\right)^{2}
$$

where $P$ and $P_{0}$ are the potential energy of the cellulose crystal under stress and nonstress energy conditions respectively; $S$ is the cross section, $l_{0}$ is the length of the crystal under non-stressed condition, and $l$ is the length of the crystal. The authors reported that the elastic modulus calculated in $4 \times 4 \times 10$ unit-cell size had smaller deviations (124-172 $G P a$ ) and were closer to the observed values (138 GPa (Nishino et al. 1995)) in comparison to the values calculated in $1 \times 1 \times 10$ unit-cell size $(89-173 \mathrm{GPa})$.

Experimental measurement of mechanical properties of cellulose began in 1962 but the work was not focused on nanocrystalline cellulose at that time. Sakurada et al. (1962) studied the crystal deformation of highly oriented fibers of cellulose I (native cellulose) by X-ray diffraction. For this purpose a Geiger counter X-ray diffractometer was used, a constant stress $(\sigma)$ was applied to the fiber bundle, and the fractional change in the length of two glucose units $\Delta I / I_{0}$, or of a net plane distance, $\Delta d / d_{0}$, was calculated from the displacement of the interference maximum:

$$
\varepsilon=\Delta \mathrm{I} / \mathrm{I}_{0}=\Delta \mathrm{d} / \mathrm{d}_{0}
$$

Table 1.1

Elastic modulus of crystalline regions of cellulose polymorphs obtained by X-ray diffraction (Nishino et al. 1995)

\begin{tabular}{cc}
\hline $\mathrm{CNC}$ & Elastic modulus $(\mathrm{GPa})$ \\
\hline $\mathrm{I}$ & 138 \\
$\mathrm{III}_{\mathrm{I}}$ & 87 \\
$\mathrm{IV}_{\mathrm{I}}$ & 75 \\
$\mathrm{II}$ & 88 \\
$\mathrm{III}_{\mathrm{II}}$ & 58 \\
\hline
\end{tabular}

Finally the elastic modulus, $E$, was calculated by $E=\sigma / \varepsilon=137 \mathrm{GPa}$. Mann and Roldan-Gonzalez (1962) also used X-ray diffraction and measured elastic moduli between 70-90 GPa for crystals of cellulose I and cellulose II, respectively. The same 
procedure was recently used by Nishino et al. (1995) to measure the elastic modulus of different polymorphs of cellulose. The results are summarized in Table 1.1. The value obtained for cellulose I is in accordance with what Sakurada et al. obtained many years before (Sakurada et al. 1962).

Raman spectroscopy is another well established technique, which has been used for measurement of the longitudinal elastic modulus of CNCs. These measurements are based on the shift in the characteristic Raman band (located at $1095 \mathrm{~cm}^{-1}$ ) that corresponds to the vibration of $\mathrm{C}-\mathrm{O}-\mathrm{C}$ bonds on the back bone of the CNCs. To conduct these measurements, CNCs are usually embedded in epoxy, and a macro-scale bending test is performed. Using this method, in 2005, Sturcova et al. (Sturcova et al. 2005) measured the elastic modulus of tunicate CNCs to be around $143 \mathrm{GPa}$. They showed that the position of the Raman band peak was linearly dependent on strain up to the value of about $0.8 \%$ and formations. After this point a plateau was formed and this was related to the weakening of the cellulose-matrix interface and as a result decrease in the stress transfer efficiency:

$$
\begin{gathered}
E=\frac{d \sigma}{d(\Delta v)} \times \frac{d(\Delta v)}{d \epsilon} \\
E_{c}=\eta_{0} \mathrm{E}_{\mathrm{f}}
\end{gathered}
$$

By substituting $d(\Delta v) / d \epsilon=2.4 \mathrm{~cm}^{-1} \%$ (from Raman band shift versus strain curves) and value of $d \sigma / d(\Delta v)=4.7 \mathrm{~cm}^{-1} / G P a$ (Eichhorn et al. 2001a and b), in Equation 1.10 , the elastic modulus of two dimensional network tunicate cellulose was calculated to be $51.1 \mathrm{GPa}$. Equation 1.8 was then used to obtain the elastic modulus of a single fibers of $\mathrm{CNC}\left(E_{f}\right)$. In this equation $E_{c}$ is the elastic modulus of a two dimensional random network of fibers and $\eta_{0}$ is the efficiency factor equal to $9 / 8 \pi$.

Table 1.2

Elastic modulus of CNCs with different origins, gained using Raman spectroscopy CNC origin Elastic modulus (GPa) Reference

\begin{tabular}{ccc}
\hline Tunicate & 143 & Sturcova et al. 2005 \\
Cotton & 105 & Rusli and Eichhorn 2008 \\
Bacterial & 114 & Hsieh et al. 2008 \\
\hline
\end{tabular}

Recently, the same technique was used to measure the elastic modulus of cotton CNCs and bacterial CNCs (Table 1.2). Hsieh et al. (2008) obtained the value of $114 \mathrm{GPa}$ for the elastic modulus of bacterial CNCs and Rusli and Eichhorn (2008) found an upper value of $105 \mathrm{GPa}$ and a lower value of $57 \mathrm{GPa}$ for the elastic modulus of cotton CNCs. Both of the values for cotton CNCs are lower than the value for tunicate cellulose measured by Sturcova et al. (2005) and the value for bacterial cellulose reported by Hsieh et al. (2008). This may be due to the smaller aspect ratio in cotton-based cellulose than the aspect ratio of other CNCs, which might have resulted in less effective stress transfer to the epoxy. 
Atomic force microscopy (AFM) is another tool that has been used for measuring the axial elastic modulus of cellulose fibers (Guhados et al. 2005; Cheng and Wang 2008; Cheng et al. 2009; Iwamoto et al. 2009). In this method the AFM tip is used to deflect a nano sized cellulose fiber, which is suspending on a groove. In the work performed by Guhados et al. (2005) sonicated suspension of bacterial cellulose fibers was placed on a silicon grating with step height of $1000 \mathrm{~nm}$ and a pitch of $3 \mu \mathrm{m}$. Fibers with diameters less than $100 \mathrm{~nm}$ were identified by AFM imaging and then using force-volume mode, force spectra were gained for an array of positions along the fibers. It was assumed that the fibers had elliptical cross sections and that they were clamped on both ends. The slope of the force spectra, $d y / d z$, that was measured along the fibers was then calculated to be:

$$
\frac{d y}{d z}=\left[1+\frac{k}{3 E I}\left(\frac{a(L-a)}{L}\right)^{3}\right]^{-1}
$$

where $k$ is the spring constant of the AFM cantilever, $I$ is the area moment of inertia, $L$ is the length of the fiber, $a$ is the position of loading with respect to one end (Figure 1.3), and $E$ is the elastic modulus of the fiber which is unknown. The elastic modulus was calculated 78 $\pm 17 G P a$, which was consistent with Voigt model (Harris 1999).

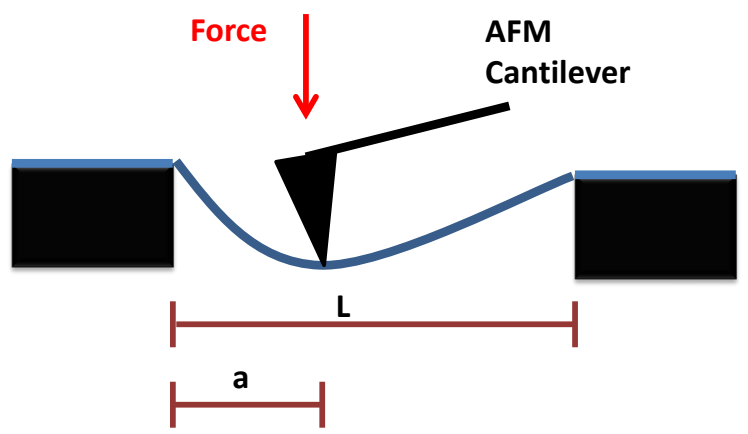

Figure 1.3 Clamped fiber with length equal to (L). Force is applied to the fiber with distance (a) from the edge using the AFM cantilever tip.

Table 1.3

Elastic modulus of CNCs with different origins, gained using atomic force microscopy Elastic modulus

$\mathrm{CNC}$ origin $(G P a)$

Reference

\begin{tabular}{ccc}
\hline Bacterial & 78 & Guhados et al. 2005 \\
Lyocell fibers & 93 & Cheng and Wang 2008 \\
Tunicate & 150.7 & Iwamoto et al. 2009 \\
\hline
\end{tabular}

Cheng and Wang used AFM and measured the elastic modulus of cellulose fibers isolated from Lyocell fibers in a three-point bending test to be $93 \mathrm{GPa}$ (Cheng and Wang 2008). Recently, Iwamoto et al. (2009) used AFM to perform three point bending tests on CNCs obtained by sulfuric acid hydrolysis of tunicate and obtained a value of $150.7 \mathrm{GPa}$. AFM seems to be a useful tool for characterization of the mechanical properties of single CNCs (Table 1.3). These bending tests require CNCs with large aspect ratio, so that they 
can be suspended over a groove and rely on good knowledge of the geometry of the sample, which is obtained from AFM imaging, and can be affected by tip broadening parameter.

Although the axial (along the axis) elastic modulus of CNCs from different sources has been under investigation for many years, there are not many reports on the transverse elastic modulus (perpendicular to major axis, $E_{t}$ ) of these materials (Jaswon et al. 1968; Tashiro and Kobayashi 1991; Lahiji et al. 2010).

In 1968, using theoretical modeling of cellulose I, Jaswon et al.(1968) estimated values of 76 and 51 and $57 \mathrm{GPa}$ for axial and two perpendicular transverse directions, respectively. Similarly, in 1991, Tashiro and Kobayashi (1991) predicated elastic moduli of 167 and 11 and $50 \mathrm{GPa}$ for axial and two transverse elastic moduli of cellulose crystals, respectively. Just recently, the first experimental data on characterization of the transverse elastic moduli of CNCs using AFM was published (Lahiji et al. 2010). Lahiji et al. used AFM measurements and a physics-based model and calculated $E_{t}=18-50 \mathrm{GPa}$ for wood-derived CNCs.

One could ask what causes such high elastic modulus in a low density polymer such as cellulose. To answer this question, we should go back to the molecular structure of cellulose. There are multiple hydroxyl groups on the cellulose chain. These can make inter-molecular and intra-molecular hydrogen bonds, which have a key role in the mechanics of this material (Figure 1.4).

When a cellulose chain goes under tension, hydrogen bonds bear the load and deform. This continues until the load reaches the strength of these bonds, and these break (yield point). After this point, $\mathrm{C}-\mathrm{O}-\mathrm{C}$ bridges come into the picture and take the majority of the load (Kroon et al. 1986; Kong and Eichhorn 2005). As a result, number and nature of the hydrogen bonds greatly affect the mechanical properties of cellulose nanocrystals. For example, Tashiro and Kobayashi (1991) showed that the intra-molecular hydrogen bonds in cellulose II fibers are weaker than those in cellulose I, but their intermolecular bonds are the same. This results in higher elastic modulus for cellulose I than II. 

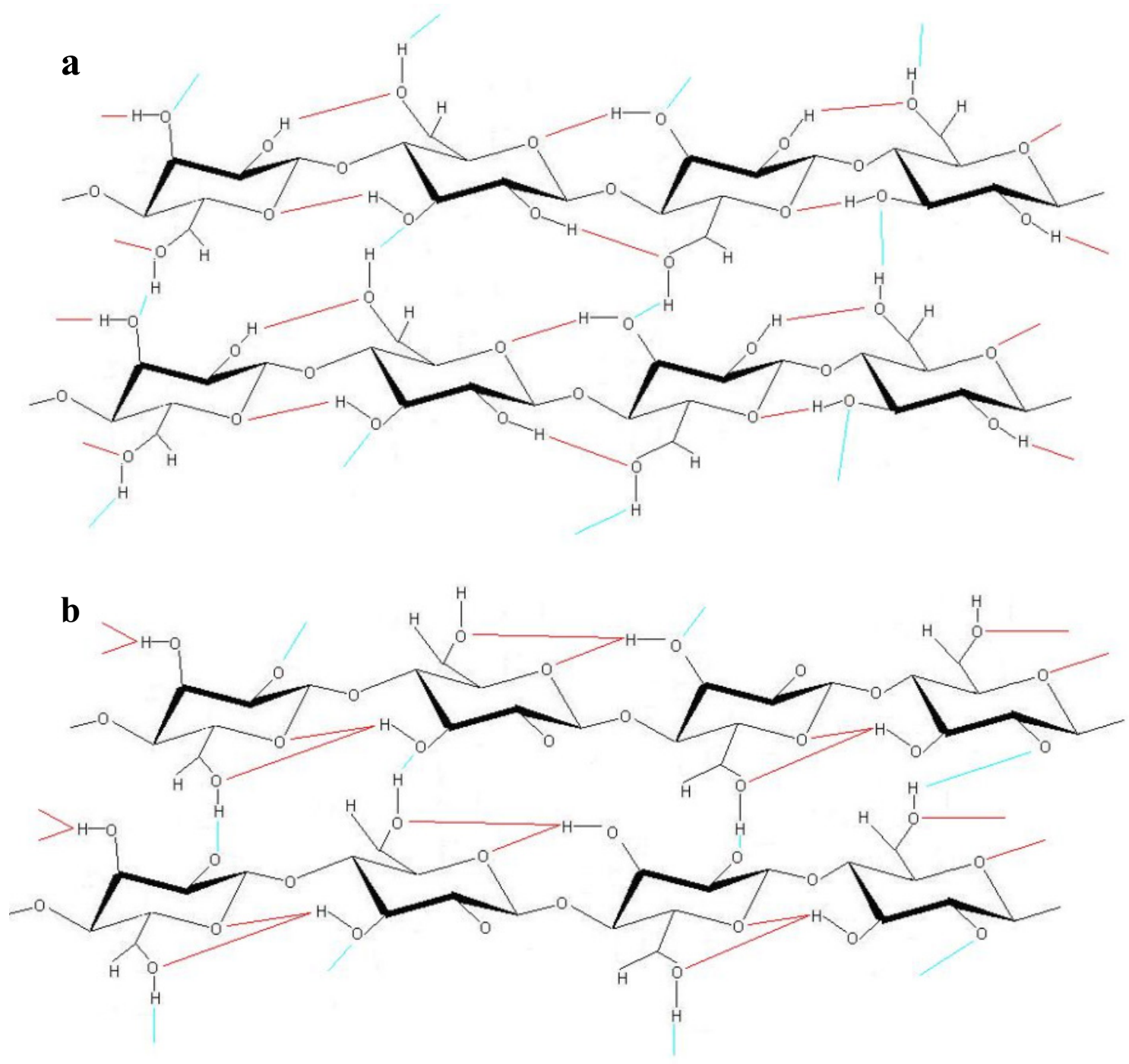

Figure 1.4 Intermolecular (blue) and intramolecular (red) hydrogen bonds in cellulose I (a) and cellulose II (b)

\subsubsection{Morphological Analysis}

Preparation conditions like hydrolysis time and temperature have great affect on the geometrical properties of resulting CNCs. Meanwhile, properties such as their diameter, aspect ratio and tendency to aggregate, have critical affects on the mechanical properties of CNC-polymer composites. Hence, it is important to examine CNC crystals before they are dispersed in polymer matrices. Various microscopy techniques can be used for this purpose. Most common techniques are:

1.1.2.1 Scanning Electron Microscopy (SEM): SEM with a field emission gun (FESEM) can be used to image CNCs (Figure 1.5). Usually a dilute solution of the sample is made, a droplet is put on a substrate and is left to air dry. In order to prevent charging and burning either low accelerating voltage (1-3 V) can be used, which will 
degrade the resolution, or the sample can be coated with conducting materials. Because of resolution limitations and instability of the sample in SEMs, it is usually difficult to obtain precise details of CNCs in these images.

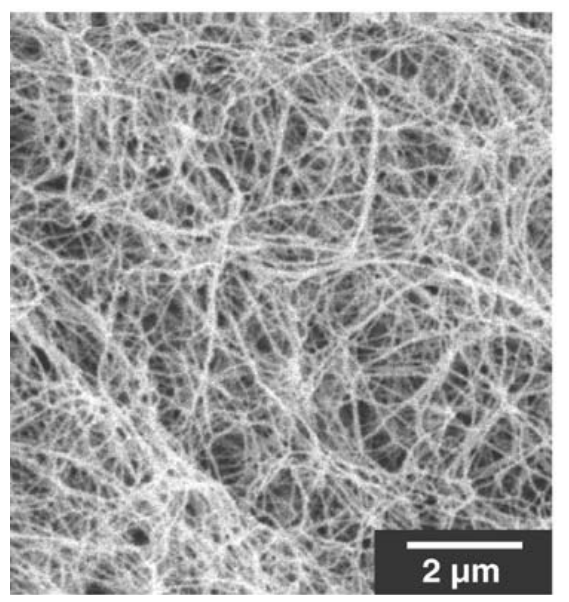

Figure 1.5 A SEM image of bacterial CNC pellicles (With kind permission from Springer Science+Business Media: Applied Physics, Bacterial Cellulose: The ultimate nanoscalar cellulose morphology for the production of high strength composites, 80 , 2005, 93-97, Nakagaito)

1.1.2.2 Transmission Electron Microscopy (TEM): In order to examine CNCs in TEM, a drop of dilute solution is put on a carbon coated grid, and let dry in air. Usually metal shadowing or negative staining is needed to improve the contrast (Figure 1.6). For instance, sample can be negatively stained by floating the grid in staining materials, such as uranyl acetate, for a few minutes. Some difficulties in TEM imaging are sample preparation techniques and beam sensitivity of the sample.

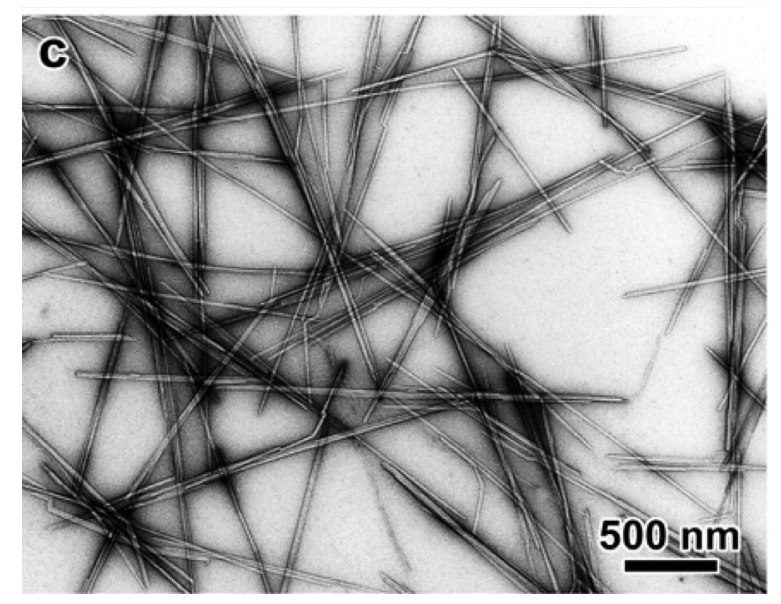

Figure 1.6 A TEM image of tunicin CNC (Reprinted with permission from (ElazzouziHafraoui et al. 2008). Copyright 2008 American Chemical Society) 
1.1.2.3 Atomic Force Microscopy (AFM): AFM in tapping mode can be used for this purpose. Again, a droplet of CNC solution in water is dried on a substrate (usually freshly cleaved mica) and studied. AFM seems to be a good alternative to electron microscopy because it does not have the limitations of low contrast and resolution, and sample preparation is much easier. The only disadvantages are the tip broadening and that scan rate that can affect the quality of the images.

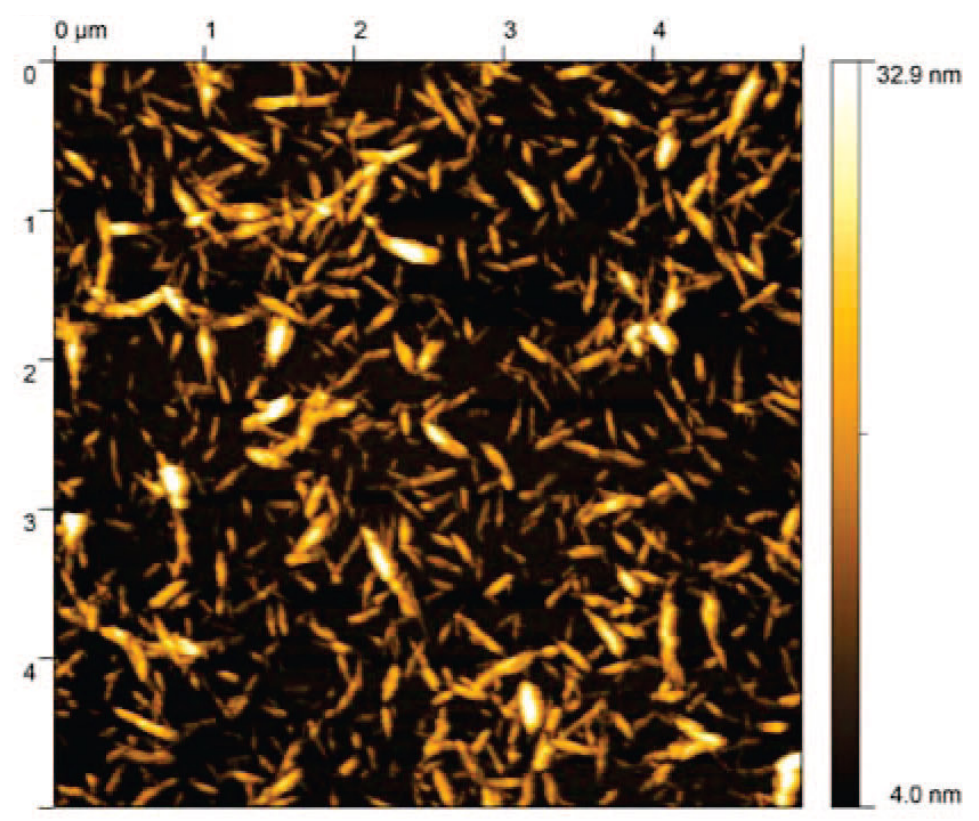

Figure 1.7 AFM image of cotton CNCs (Reprinted with permission from (ElazzouziHafraoui et al. 2008). Copyright 2008 American Chemical Society)

\subsection{Cellulose Based Composites}

Nanocomposites have attracted great attention in the scientific community because of the significant enhancement in the base materials by the addition of nano-reinforcements. Primary difference of nanoreinforcements with microreinforcements is their size. Because of their smaller diameter, nanomaterials have much higher surface to volume ratio. This is of major importance when these materials are used as reinforcements in polymers. The significant increase in number and surface area of the crystals at nanoscale contributes to the enhanced physical properties in the polymer matrix and extensive publications exist on the mechanical properties (Wung et al. 1991; Kojima et al. 1993; Vaia et al. 1993; Giannelis et al. 1996), thermal stability (Kojimia 1997), superconductivity (Nagaoka et al. 1984), and electrical magnetic behavior (Schmidt et al. 1991; Carotenuto et al. 1996) of various nanocomposites.

Although a wide variety of synthetic nano reinforcements have been used to reinforce polymer matrices (Koo et al. 2003; Peng et al. 2005; Hongmei et al. 2007; Mi et al. 2007), due to the current environmental issues, investigations are being shifted toward the use of natural reinforcements (Mohanty et al. 2001; Shibata et al. 2004). Amongst various resources available, cellulose in the form of cellulose nanocrystals (CNCs) has attracted 
different research groups around the world since 1995 (CNCs were used to reinforce a polymer matrix for the first time by Favier et al. in 1995).

\subsubsection{Mechanics of CNC Composites}

In a composite system reinforcements are used to carry the mechanical loads, improve the properties and lower the cost of the final product. On the other hand, matrix, which in our case is a polymer, is there to increase the toughness, to disperse reinforcements and to transfer the load to the reinforcements. Nano reinforcements are used widely these years because they have less defects and higher surface area compared to larger sized materials. Thus, they bring additional improvements and unique characteristics to the final product, at lower reinforcement content levels as compared to micro- and macro-sized materials.

Like all composite systems, the properties of cellulose nanocomposites depend on the properties, the volume fraction, and the spatial arrangement of the matrix and the reinforcement. Dispersion of CNCs in the polymer matrix is not an easy procedure, and evenly distributed reinforcement is crucial for enhancement of the mechanical properties of composites. This can be specially challenging in the cases that polymer matrix is hydrophobic (as opposed to hydrophilic CNCs). Without changing the surface activity and dispersion qualities of $\mathrm{CNCs}$ the ultimate mechanical properties of the nanocomposite may not be much different or they may even be worse compared to the pure polymer.

On the other hand, the main factors that dictate the mechanical properties of CNCpolymer composites are:

The aspect ratio of the CNCs $(L / D)$ : This ratio depends on the origin of the crystals and the higher it is the better the mechanical properties will be (Orts et al. 2005; Lu et al. 2006; Kvien et al. 2007).

CNCs from different origins will have diverse size distributions, surface properties and more importantly different aspect ratios. All of these have fundamental influence on the mechanical properties of the final composition. Figure 1.8 shows differences in the elastic modulus of composites made of starch (with elastic modulus less than $2 \mathrm{GPa}$ ) and 5 wt.\% CNC from various origins. Similarly, Rusli et al. (2011) compared the stress transfer qualities in polymer composites reinforced with CNCs from tunicate and cotton. They observed the greatest ammount of stress transfer in composites reinforced with tunicate $\mathrm{CNCs}$, which have much higher aspect ratios. 


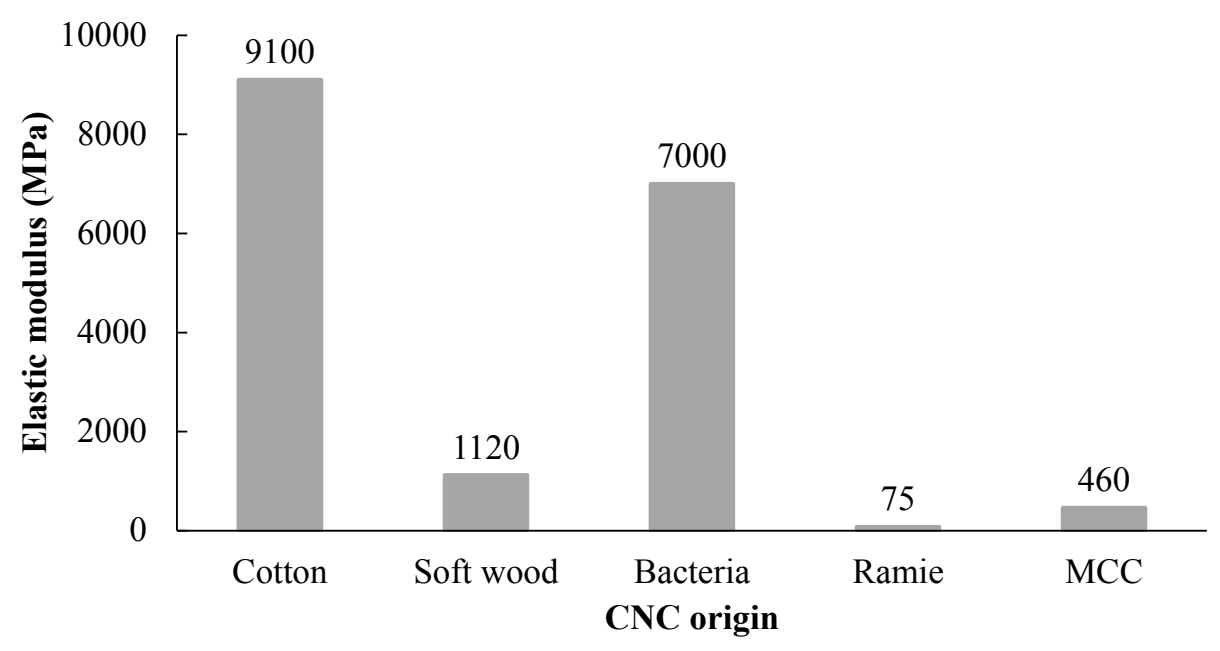

Figure 1.8 Effect of CNC origin on the Young's modulus of CNC/starch composites (Orts et al. 2005 Lu et al. 2006; Kvien et al. 2007)

The processing method: Extrusion, hot pressing and evaporation are some techniques that are used. These techniques affect the orientation of the reinforcement, the $\mathrm{CNC} / \mathrm{CNC}$ interactions and they may cause breakage of the crystals (changing the aspect ratio). Processing methods affect the final mechanical properties of the nanocomposite (Gong et al. 2011). For instance, in the case of CNC/latex composites, it has been shown that the efficiency of the processing methods is: extrusion $<$ hot-pressing $<$ evaporation (Hajji et al. 1996).

The resulting competition of matrix/CNC and $C N C / C N C$ interactions: As opposed to the case in cellulose nanocomposites, the preferred condition in most of composite materials is that there is maximum interaction between matrix and the reinforcement. On the other hand in $\mathrm{CNC}$ composite systems the $\mathrm{CNC} / \mathrm{CNC}$ interactions should be predominant, so that a 3D network of CNCs is made to keep the weak polymer in place and yield in higher stiffness and thermal stability in the resulting material (Azizi Samir et al. 2005). Percolating threshold, above which this 3D network is formed, is the critical volume fraction $\left(v_{R c}\right)$, which separates the local and infinite communication of the crystals. It depends on the particle interactions and orientation and aspect ratio of CNCs and it can be calculated using Eq. 1.1 (Azizi Samir et al. 2005). Based on this equation, percolating threshold for composites with high aspect ratio fillers can be as low as $1 \%$.

$$
v_{R c}=\frac{0.7}{L / d}
$$

Favier et al. (1997) predicted the reinforcing effect of CNCs based on the percolating theory, and calculated the elastic modulus of composite $\left(E_{c}\right)$ as bellow: 


$$
E_{C}=\frac{\left(1-2 \psi+\psi v_{F}\right) E_{M} E_{F}+\left(1-v_{F}\right) \psi E_{F}^{2}}{\left(1-v_{F}\right) E_{F}+\left(v_{F}-\psi\right) E_{M}}
$$

where the subscripts $M$ and $F$ refer to the matrix and filler, respectively. $\Psi$ and $E_{F}$ correspond to the volume fraction and modulus of the stiff percolating network (CNCs), respectively. $\Psi$ can be written as:

$$
\begin{array}{ll}
\psi=0 & \text { for } \mathrm{v}_{\mathrm{F}}<\mathrm{v}_{\mathrm{Rc}} \\
\psi=v_{F}\left(\frac{v_{F}-v_{R c}}{1-v_{R c}}\right)^{b} & \text { for } v_{F} \geq v_{R c}
\end{array}
$$

$v_{F}$ and $v_{R c}$ correspond to the volume fraction of the $\mathrm{CNC}$ and the critical volume fraction at the percolation threshold respectively and $b$ is the corresponding critical exponent and is equal to 0.4 in a three-dimensional network.

Although $\mathrm{CNC} / \mathrm{CNC}$ interactions need to be predominant, in order for the stress to be effectively transferred to the reinforcement, good adhesion between CNC and matrix is vital (Heux et al. 2000; Grunert and Winter 2002; Gopalan Nair et al. 2003; Rusli et al. 2011) and not all polymers will have good adhesion to cellulose. This is where interphase comes into the picture. Interphase is a gradient region that extends from the matrix to the reinforcement surface and transfers the loads to the reinforcements. The properties of the interphase (mechanical properties, thickness, etc.) is one of the parameters that define the ultimate properties of the composite system (Hashin 1990; Moon et al. 2011).

In order to optimize the interfacial bonding of $\mathrm{CNC}$ and polymer matrices, some modifications are possible. These include physical methods (for example changes in the structure and surface of CNCs by coating them with surfactant (Heux et al. 2000)) and chemical modifications (ex. grafting them with hydrophobes (George et al. 2001; Grunert and Winter 2002; Gong et al. 2011). One drawback to these procedures is that modified CNCs have less reinforcing effects than not modified ones. This is due to the destruction of 3D network of CNCs, as a result of changes in hydrogen bondings during these modifications. Another possibility can be the less efficient stress transfer from the polymer to CNCs (Grunert and Winter 2002; Gopalan Nair et al. 2003).

Following is a short review on the studies performed to characterize the tensile, nanoindentation, creep and thermo mechanical properties of CNC-based nanocomposites.

\subsubsection{Tensile Properties}

Tensile tests are usually performed in universal test machines on pure polymer matrix and nanocomposite samples, which are molded or cut in dog-bone or rectangular shapes. Important macro-scale mechanical properties such as elastic modulus, tensile strength, yield strength and ductility, can be derived from the load-displacement or stress-strain curves. Furthermore, by studying the shape of these curves one can gain useful information about the morphology of the ultimate nanocomposite and the dispersion quality if the reinforcements. For instance no necking can be a representative of well dispersion of $\mathrm{CNC}$ in the polymer. 
Several reviews exist on the mechanical properties of CNC-polymer composites (Azizi Samir et al. 2005; Eichhorn et al. 2010; Hubbe et al. 2008; Oksman and Sain 2006). Compared to non-reinforced polymers, CNC-based composites show improvements in tensile modulus and yield strength (Azizi Samir et al. 2005; Yongshang et al. 2005; Choi and Simonsen 2006; Marcovich et al. 2006; Cao et al. 2007; Kvien et al. 2007; Lapa et al. 2007; Noorani et al. 2007; Petersson et al. 2007; Auad et al. 2008; Roohani et al 2008; Cherian et al. 2011; Qua and Hornsby 2011; Singha et al. 2011). As an example, Figure 1.9 depicts the changes in mechanical properties of two different polymers (soy protein isolate (SPI) and polyvinyl alcohol (PVA)) before and after addition of CNCs derived from cotton linters. Although the total trend is that the tensile strength and elastic modulus increase by adding CNCs to pure polymers, the amount and quality of these changes strongly depend on the polymer and its interaction with the crystals.

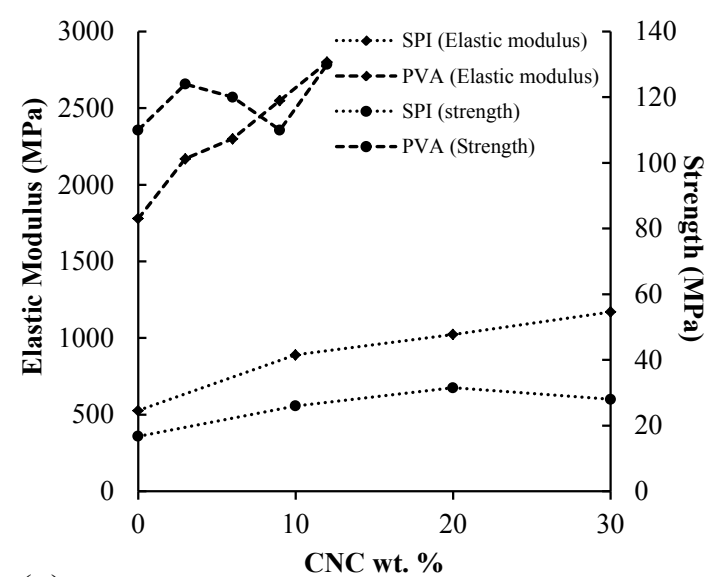

(a)

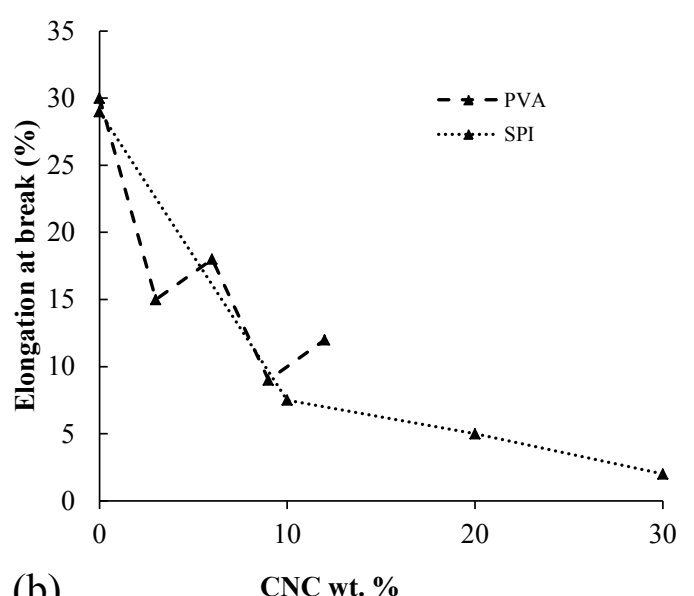

(b)

Figure 1.9 Change in elastic modulus and tensile strength (a) and elongation at break (b) of soy protein isolate (SPI) and polyvinyl alcohol (PVA) filled with different amounts of CNC derived from cotton linter (Wang et al. 2006; Roohani et al. 2008)

In general, an optimum $\mathrm{CNC}$ content is desired in order for the percolation to occur and to achieve the desired mechanical properties (Choi and Simonsen 2006; Wang et al. 2006; Roohani et al. 2008). After this optimum value, the addition of CNC may result in the reduction of tensile strength and/or elastic modulus due to phase separation which occurs because of agglomeration of the nanocrystals (see the strength data on Figure 1.9). This optimum CNC content depends on the characteristics of the polymer matrix and its interaction with nanocrystals. The tensile strength and/or elastic modulus decrease with addition of more cellulose and this is due to phase separation which occurs because of agglomeration of the nanofillers.

Elongation at break mostly decreases with addition of $\mathrm{CNCs}$, meaning that the material is modified from being ductile (with long deformation after yield until fracture) to very brittle (with almost no plastic deformation after yielding point) (Figure 1.9b). Addition of a hard filler to a soft matrix results in higher strength at the cost of ductility. 
The reduction in elongation at break is a sign of good $\mathrm{CNC} / \mathrm{CNC}$ and formation of a rigid $3 \mathrm{D}$ network, which restricts the motion of the polymer matrix.

Experimental conditions such as temperature and relative humidity have been shown to have great effects on tensile test results. For example as it seen in Figure 1.10, increase in the relative humidity $(\mathrm{RH})$, tends to degrade the mechanical properties of cellulose nanocomposites with hydrophilic matrices (Dufresne et al. 2000; Wang et al. 2006). This is because at high humidity levels, hydrophilic polymer and CNCs absorb large amounts of water. As a result the $\mathrm{CNC} /$ matrix and $\mathrm{CNC} / \mathrm{CNC}$ interactions decline, $\mathrm{CNCs}$ become surrounded by a week and soft phase, and the reinforcing effect of CNCs diminishes.

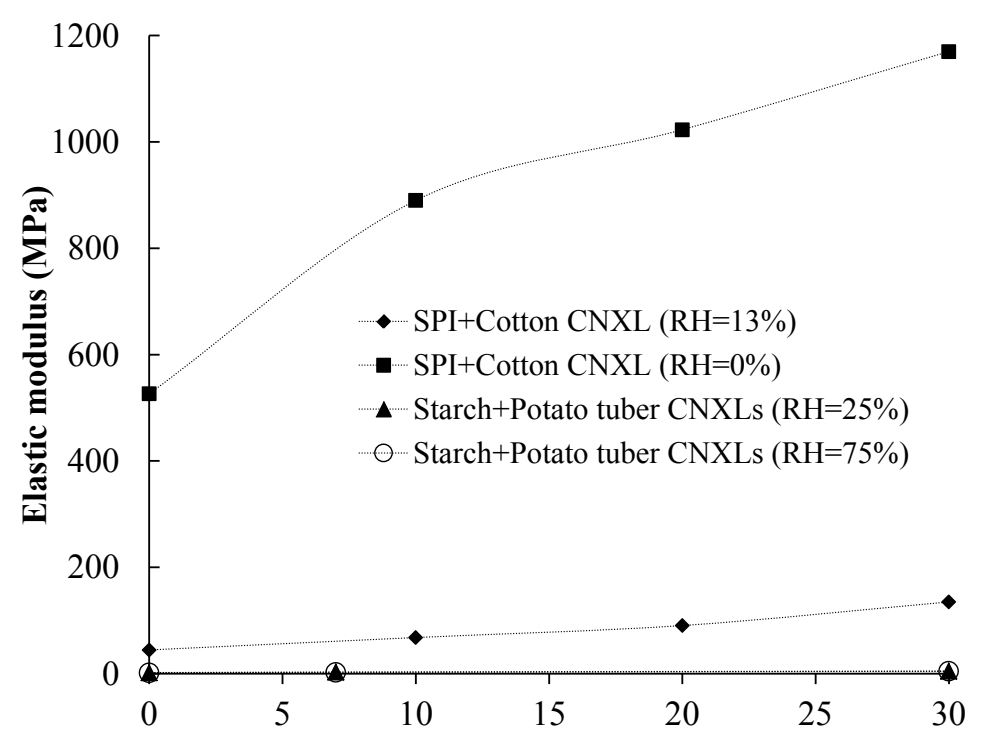

CNC wt.\%

Figure 1.10 Change in elastic modulus of soy protein isolate (SPI) and Starch filled with CNC at various relative humidity (RH) (Wang et al. 2006; Dufresne et al. 2000)

\subsubsection{Nanoindentation Studies}

Another technique for mechanical characterization of polymer composites is nanoindentation. Nanoindentation is similar to macro-hardness tests but is done at the nano-scale. Typically a diamond indentation tip with known elastic modulus and hardness is pressed into the surface of the sample, and the applied normal load $(P)$ and the indentation height into the surface $(h)$ are measured continuously during the loading and unloading. A schematic load-displacement curve is shown in Figure 1.11. The elastic modulus $(E)$ and hardness $(H)$ are then calculated using the slope of the unloading part of the curve $(S)$ and the equations bellow:

$$
\begin{gathered}
S=\frac{d P}{d h}=\frac{2}{\sqrt{\pi}} E_{r} \sqrt{A} \\
\frac{1}{E_{r}}=\frac{\left(1-v^{2}\right)}{E}+\frac{\left(1-v_{i}^{2}\right)}{E_{i}}
\end{gathered}
$$




$$
H=\frac{P_{\max }}{A}
$$

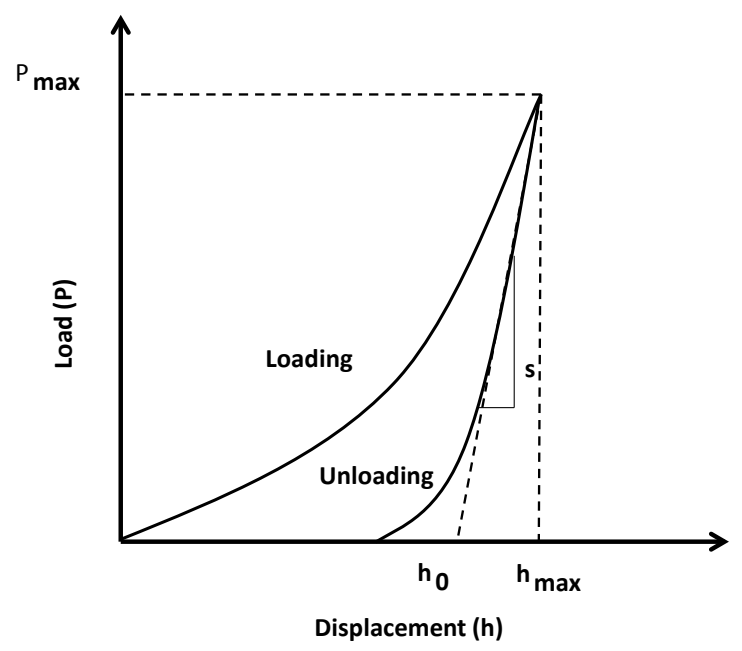

Figure 1.11 Typical load-displacement curve

$S$ is the slope of the load-displacement curve, which is obtained by fitting a second order polynomial function to the curve and differentiation $\left(\frac{d P}{d h}\right) . A$ is the projected contact area. As opposed to conventional indentation techniques, here the contact area is measured indirectly using the indentation height and the known geometry of the indenter. $E_{r}$ is the reduced elastic modulus (the measured elastic modulus includes effects from both the specimen and the indenter), $v_{\mathrm{i}}, v$ and $E_{i}, E$ are the Poisson's ratios and the elastic moduli of the indenter and the sample respectively (Oliver and Pharr 1992).

Although several nanoindentation experiments have been reported for cellulose nanofiber reinforced polymer composites (Zimmermann et al. 2005; Lee et al. 2007), not many reports exist on nanoindentation studies of CNC composites. Two interesting applications for nanoindentation in the field of polymer composite materials are: characterization of the nanomechanical properties at the surface and comparison to the bulk mechanical properties and characterization of the mechanical properties at the interphase.

Care should be taken during analysis of nanoindentation results for polymer composites. There are some debates that the famous Oliver-Pharr method (Oliver and Pharr 1992), should not be used for polymer materials (Tranchida et al. 2007). One should be careful when comparing results from nanoindentation tests on different materials, using different tips, techniques and in different laboratories. There are some phenomenon which can affect the test results. For example: Pile up is bulging out of the free surface of the material, which changes the contact area, and ultimately the $\mathrm{E}$ and $\mathrm{H}$ calculations. Viscoelasticity characteristics of polymers affects the unloading curves, and results in what is called a "nose" on the curves, where the indentation height increases while the load is being decreased. This results in negative values for contact stiffness $(S)$. Finally the morphology of the indentation surface is vastly affected by sample 
preparation techniques that can result in different microstructures with different mechanical properties and/or various microscopic roughnesses. These ultimately give various $E$ and $H$ for various positions on one specific sample and increase the standard deviations from the average values.

\subsubsection{Thermo-Mechanical Characterization}

Thermo-mechanical characteristics of CNC composites are mostly investigated using dynamic mechanical analysis (DMA), where the storage modulus (tensile: $E^{\prime}$ or shear: $G^{\prime}$ )

and the position of $\tan \delta$ peak (the loss factor, $\tan \delta=\frac{E^{\prime \prime}}{E^{\prime}}$ where $E^{\prime \prime}$ is the loss modulus) are compared in the pure polymer and in composites with different filler contents. DMA is mostly performed in tensile $\left(E^{\prime}\right)$ or sheer $\left(G^{\prime}\right)$ mode, in a wide range of temperatures around the glass transition temperature of the polymer. These tests are usually done with a constant frequency (e.x. $1 \mathrm{~Hz}$ ) and they provide information about the visco-elastic properties of materials.

Generally cellulose nanocomposites have been found to be more thermally stable than the matrix alone. Again, enough $\mathrm{CNC}$ content causes a continuous 3D network (percolation), which reduces the mobility of polymer matrix and as a result the storage modulus $\left(E^{\prime}\right.$ or $\left.G^{\prime}\right)$ increases and tan $\delta$ peak broadens. Above melting temperature $\left(T_{m}\right)$ is where CNCs have the most effect on thermo mechanical properties of the composites. As opposed to pure polymer, here storage modulus does not drop by increase in temperature, thus performance at high temperatures is improved and thermal stability is brought to the ultimate material up to degradation temperature of cellulose (around $500 \mathrm{~K}$ ).

Another thermo mechanical observation in nanocomposite polymers filled with CNC is increase in glass transition temperature $\left(T_{g}\right)$. This phenomenon is related to change in the kinetics of the glass transition due to the presence of the nanocrystals and also increase in cross linking and as a result decrease in the mobility of the polymer chains. Broad amount of work has been concentrated on the thermo mechanical properties of CNC polymer composites, for instance see: Favier et al. 1995; Helbert et al. 1996; Grunert and Winter 2002; Azizi Samir et al. 2004-a, b; Choi and Simonsen 2006; Ljungberg et al. 2006; Lu et al. 2006; Marcovich et al. 2006; Wang et al. 2006; Alemdar and Sain 2007; Bodenson and Oksman 2007; Brown and Laborie 2007; Cao et al. 2007; Kvien et al. 2007; Peterson et al. 2007; Wang and Sain 2007; Auad et al. 2008; Habibi and Dufresne 2008; Roohani et al. 2008; Abdul Khalil et al. 2011; Ben Mubarak et al. 2011; Goffin et al . 2011.

\subsubsection{Morphological Analysis}

The dispersion quality of $\mathrm{CNC}$ through the polymer matrix has great effects on the nanocomposite ultimate properties. As mentioned before, hydrogen bonding is the main reason for formation of the rigid 3D network of cellulose crystals above percolating threshold, which holds the polymer matrix and improves its mechanical and thermal properties. Meanwhile, these bonds can bring difficulties in $\mathrm{CNC}$ dispersion, cause aggregation of CNCs, and degrade the ultimate properties. 
Since the size of CNCs is smaller than the light wavelength, if the pure polymer is transparent and the final composite comes out opaque, one can conclude that there are agglomerations of CNCs, simply by eye observations. Some of the techniques used for characterization of the filler dispersion in nanocomposite materials are: optical microscopy (OM), SEM, TEM, AFM, small angle X-ray scattering (SAXS) and wide angle $\mathrm{x}$-ray diffraction (WAXD). Here we discuss three most common techniques (OM, SEM and TEM). OM uses light, SEM uses electrons to scan the surface of the specimen and TEM passes electrons through a thin slice of the specimen.

1.2.2.1Optical Microscopy (OM): Observation of the solid surface of polymer nanocomposites in an $\mathrm{OM}$ can results in some qualitative information about the $\mathrm{CNC}$ dispersion. If $\mathrm{CNCs}$ are not individualized and dispersed well, their aggregate will appear in the image (Figure 1.12).
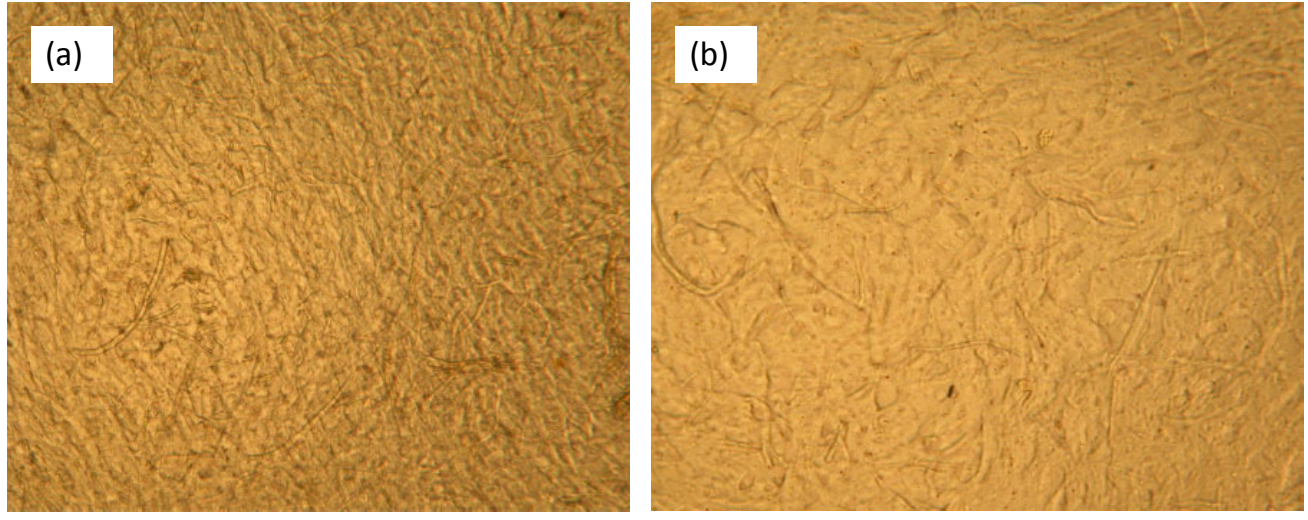

Figure 1.12 Optical Microscopy images of CNC-PVA composites (a) reinforcement without coating; (b) reinforcement coated with ethylene-acrylic oligomer. The dispersion is better in $b$, where less aggregates and filler with smaller diameters are observed (Reprinted from Composites Science and Technology, 67, Wang and Sain, Isolation of naofibers from soybean source and their reinforcing capability on synthetic polymers, 2521-2527, Copyright 2007, with permission from Elsivier)

1.2.2.2 Scanning Electron Microscopy (SEM): The fracture surface of polymer composites (obtained either by cryo fracturing in liquid nitrogen temperature or from tension tests), are observed in SEM and compared to the surface of pure polymer. Addition of CNCs to polymers alters their fracture mechanism. The fracture surface of thermoplastics is usually featureless, flat and smooth. After CNCs are added, up till the optimum point, rigid CNCs act as obstacles for movement of dislocations and cracks and make them change path. As a result the fracture surface comes out to be rough and irregular with coarse slip planes. After this point, addition of more $\mathrm{CNC}$, results in their agglomeration and inferior mechanical properties and this coincides with voids, wrinkles and crystals being pulled out of the polymer matrix.

Usually CNCs appear as white dots during the SEM imaging due to the highly nonconductive nature of the nanocrystals (Figure 1.13). The concentration of these dots increases as the reinforcement content increases in the nanocomposite. When CNCs are 
well dispersed and there is good adhesion between them and the polymer matrix fractography of the fracture surface reveals no sign of agglomeration of nanocrystals.
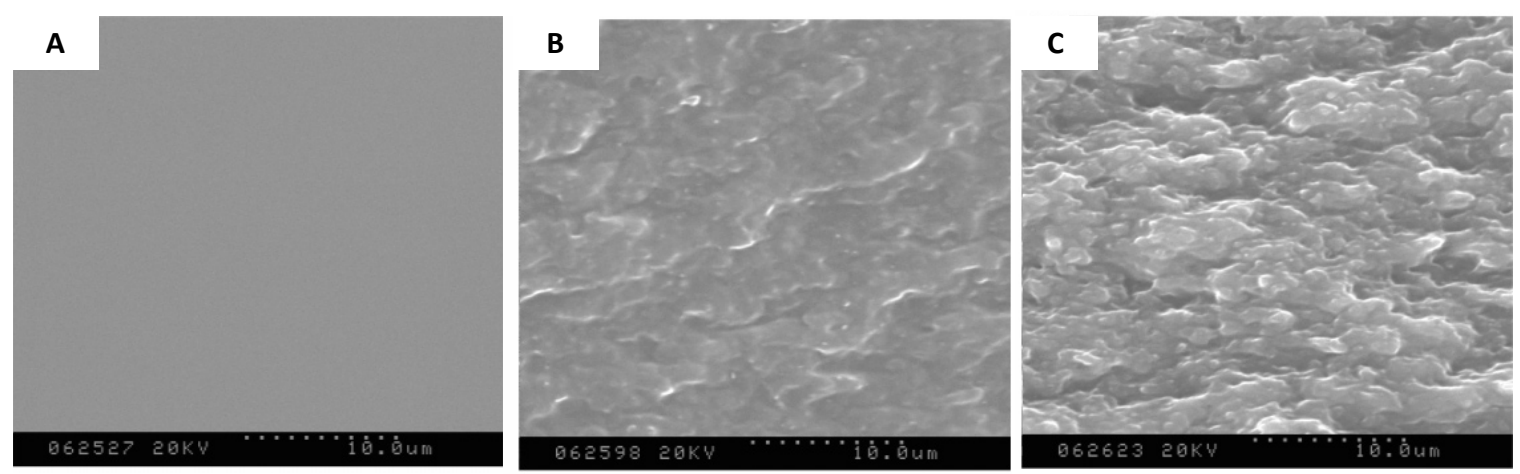

Figure 1.13 SEM images of the polyurethane filled with different wt. \% of CNC: a) $0 \mathrm{~b}$ ) 20 c) 30. In contrast with the fracture surface in (a) which is featureless, the fracture surfaces are rough in (b) and (c) and the white dots on them are the CNCs (Reprinted with permission from (Cao et al. 2007). Copyright 2007 American Chemical Society)

1.2.2.3 Transmission Electron Microscopy (TEM): In this case, very thin $(<100 \mathrm{~nm})$ slices of the specimen are prepared using ultra microtome with a diamond knife. These slices are then mounted on carbon coated grids and studied in TEM. Because CNC and the matrix are both polymers, CNCs need to be stained (usually negatively, using materials such as uranyl acetate) to improve the contrast. Because of difficulties with respect to samples preparation and also cost, TEM is not used as widely as SEM is.

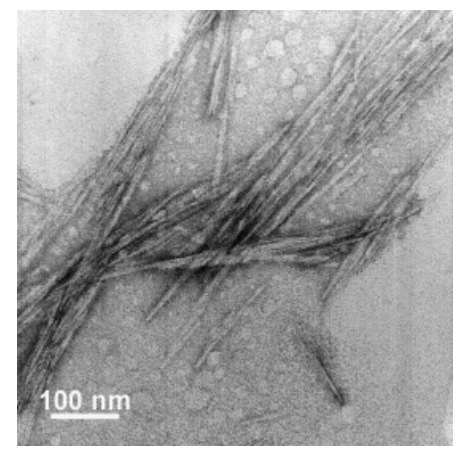

Figure 1.14 TEM images of CNC-PLA composite (Reprinted with permission from (Kvien et al. 2005). Copyright 2005 American Chemical Society)

\subsection{Order of Dissertation Chapters}

Cellulose has been under investigation for many years as a potential reinforcement in polymer composites systems. However, there are several unresolved issues that can adversely affect the properties of cellulose-based composites. Some of these are the interphase properties and the effect of $\mathrm{CNC}$ size on the mechanical properties of nanocomposites. The main purpose of this research was to investigate the 
nanomechanical properties of cellulose-based nanocomposites. To meet this pbjective, the dissertation was arranged in the below order:

First, the effect of CNC source and preparation procedures on their size are discussed (Chapter 2). Because cellulose is hydrophylic, one of the main challenges in preparation of useful cellulose composites is to improve the cellulose dispersion in a non polar polymer matrix. SEM and TEM are most commonly used techniques for characterization of dispersion quality of cellulose in polymer matrices, but these techniques only provide information from the fracture surface or a very small portion of the material. In Chapter 3 , a novel technique was used to make a correlation between cellulose dispersion and mechanical properties of a non polar polymer composite. We used X-ray Ultra Microtomography (XuM) and showed that dispersion of cellulose in the polymer matrix greatly affects the mechanical properties in macro scale. The stress in composite systems is transferred to the reinforcement through the interface. This is another indication of the fact that sufficient interfacial adhesion between cellulose and the polymer in necessary.

In order to improve the interfacial properties, in next step a hydrophilic polymer, poly(vinyl alcohol) (PVA), was selected as the matrix and various amounts of CNCs were used as reinforcement (Chapter 4). Mechanical properties of CNC-PVA composites were characterized using macroscale tensile tests. Additionally, nanomechanical properties of these composites were characterized using nanoindentation in AFM. This was performed to make a correlation between nanoscale mechanical testing and those tests at macroscale.

There is usually an optimum amount of $\mathrm{CNC}$ in a polymer composite that results in the desired macro-mechanical properties. In order for the mechanical percolation to happen $\mathrm{CNC} / \mathrm{CNC}$ interactions have to be predominant. On the other hand sufficient $\mathrm{CNC} /$ matrix interaction at the interphase is needed in order for the load to be transferred to CNCs. Thus the CNC-PVA composites with the best tensile test results were selected for interphase studies in Chapter 5. We used AFM and characterized the quantitative nanomechanical properties of the interphase. Interestingly, a direct correlation was observed between the CNC diameter and the interphase thickness. It was concluded that the interphase thickness was higher for CNCs with larger diameters (size effect).

The size effect observed in Chapter 5 and the fact that the macroscopic mechanical behavior of cellulose nanocomposites is strongly affected by the mechanical properties of individual CNCs, led us to the studies in Chapter 6. Thus, the size-dependant transverse elastic moduli of individual CNCs prepared from two different sources were measured using nanoindentation in AFM. Size scale effect on the nanomechanical properties of cellulose nanocrystals was reported and several reasons such as change in crystal structure $\left(\mathrm{I}_{\alpha} / \mathrm{I}_{\beta}\right.$ ratio) and crystallinity were proposed. In order to investigate these hypotheses, transverse elastic moduli of CNCs with different surface properties (carboxylated and sulfated) were characterized (Chapter 7). Additionally X-ray diffraction was used to study the changes in cellulose crystal structure before and after these surface modifications. 


\section{Preparation of Cellulose Nanocrystals}

\subsection{Introduction}

It has been known for more than fifty years that acid hydrolysis of natural cellulose yields in nano-sized crystals known as cellulose nanocrystals (CNCs) (Ranby 1951). Due to their good mechanical properties and renewable origins, CNCs have gained increasing interest, especially in the field of polymer nanocomposites (Iwamoto et al. 2009).

Four main groups of cellulose sources have been used to make CNCs: wood (BeckCandanedo et al. 2005), agricultural byproducts (Beck-Candanedo et al. 2005)), animal cellulose including sea tunicates (Angles and Dufrense 2001; Sturcova et al. 2005), and bacteria (Grunert and Winter 2002). The properties of the resulting CNCs depend on the cellulose origin (i.e. their degree of crystallinity), as well as the hydrolysis conditions (Bondenson et al. 2006). For example, longer reaction time results in shorter CNCs with higher surface charges (Beck-Candanedo et al. 2005).

The main factors that control the ultimate properties of CNC-polymer composites are the aspect ratio and surface chemistry of the CNCs. CNCs from tunicin and bacteria tend to have the highest aspect ratios. Tunicin whiskers are typically reported to have 10-20 $\mathrm{nm}$ cross section and $100 \mathrm{~nm}$ to few $\mu \mathrm{m}$ length (Favier et al. 1995; Terech et al. 1999). Bacterial cellulose is reported to have $5 \mathrm{~nm}$ to $50 \mathrm{~nm}$ cross section and $100 \mathrm{~nm}$ to few $\mu \mathrm{m}$ length (Toko et al. 1998; Grunert and Winter 2002).

The surface chemistry depends on the medium used to hydrolyze the sources. For example, $\mathrm{HCl}$ gives rise to an uncharged surface (Azizi Samir et al. 2005) while $\mathrm{H}_{2} \mathrm{SO}_{4}$ creates a charged sulfonate surface (Gardner et al. 2008) and tends to be more stable in water (Araki et al. 1998). Numerous modifications of the surfaces have been made to make the surfaces more compatible with a desired medium. Most of these methods make use of the primary hydroxyl group on cellulose, either directly or by first converting it to a carboxyl group. Some examples include silylation (Abdelmouleh et al. 2002), amide coupling (Araki et al. 2001), graft copolymerization (Mohanty et al. 2000), and esterification (Tserki et al. 2005; Huang et al. 2006).

\subsection{CNC Preparation and Characterization}

Figure 2.1 shows the steps taken for preparation of CNCs from different sources. Cellulose $(2.5 \mathrm{~g}$ of finely chopped cotton batting, $125 \mathrm{~mm}$ Whatman \#1 filter paper, or Sigma Aldrich MCC Batch 07422AJ) was slowly added to ice-cold concentrated sulfuric acid ( $1.0 \mathrm{~g}$ cellulose to each $8.0 \mathrm{~g}$ of concentrated sulfuric acid) being stirred with a magnetic stirrer. The cellulose addition was sufficiently slow so that the exotherm did not exceed $\sim 5{ }^{\circ} \mathrm{C}$. Once the addition was completed the suspension was heated with stirring for 50 minutes at $60{ }^{\circ} \mathrm{C}$. After 50 minutes of acid hydrolysis, the reaction was allowed to cool to room temperature. De-ionized water was then slowly added to reach the $\mathrm{pH}$ of $\sim 6$. This suspension was then subjected to centrifugation (IEC DPR-6000 centrifuge) at 4200 rpm, 4-5 repetitions, for 4 to $7 \mathrm{~min}$, followed by decanting the supernatant. This process 
was repeated until the wash water possessed a neutral $\mathrm{pH}$. This suspension was then placed in dialysis bags ( $1 A$ cutoff), and washed against de-ionized water for several days.

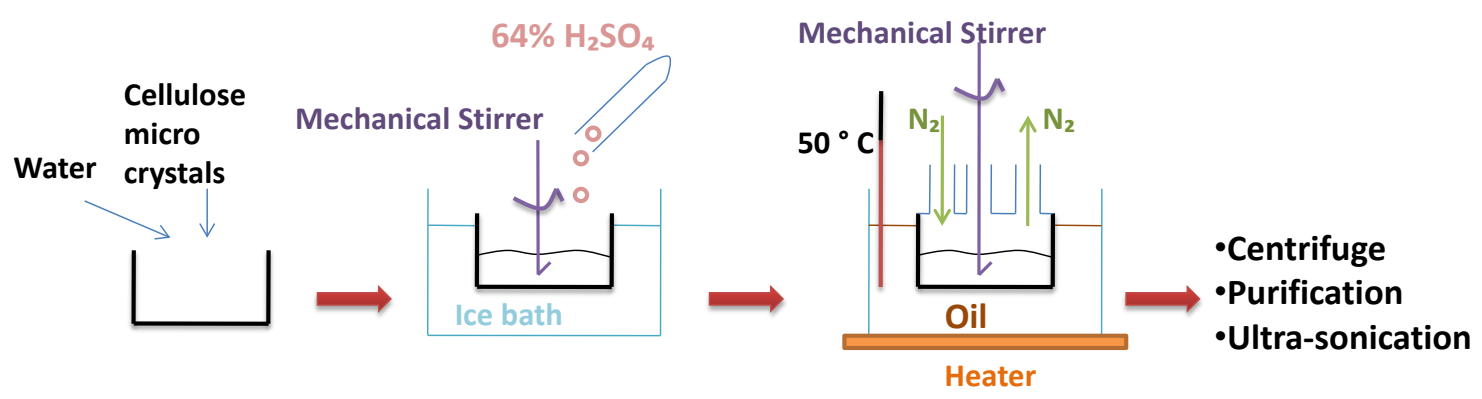

Figure 2.1 Schematic procedure for acid hydrolysis of commercially available microcrystalline cellulose. After the acid (ex. $\mathrm{H}_{2} \mathrm{SO}_{4}$ ) is added slowly to the water solution, it should be heated to certain temperatures and kept for certain times. Afterwards, using centrifuge, dialysis against distilled water, sonication and maybe ultra filtering CNCs are separated.

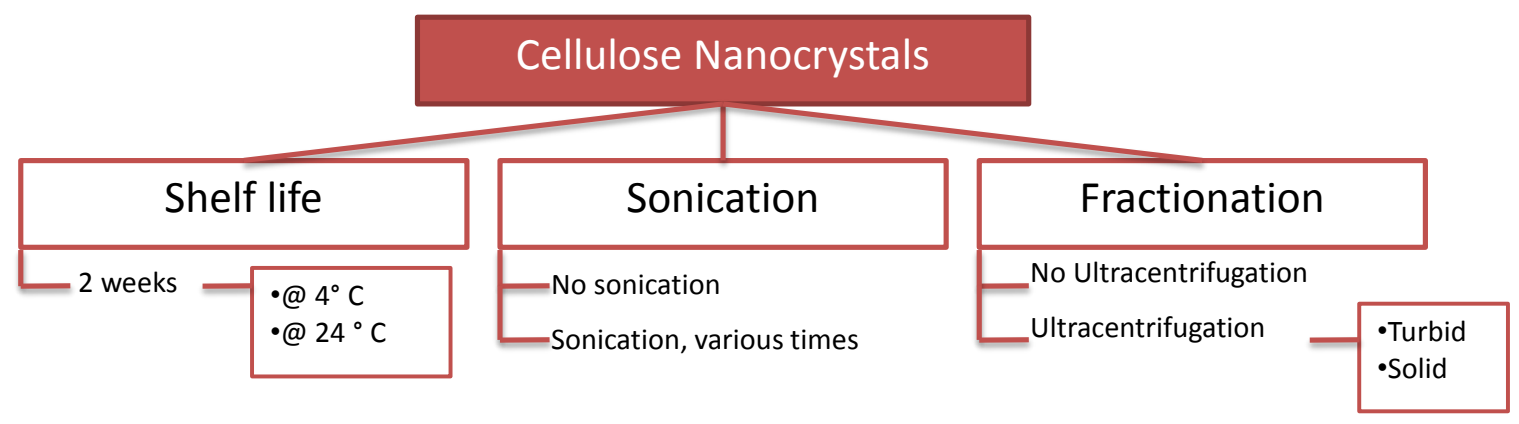

Figure 2.2 Importance of three variables were studied on the resultant size of cottonCNCs. This included shelf life (at two different temperatures), sonication (various times), and fractionation using ultracentrifugation.

After the dialysis was complete, the final CNCs from different sources were characterized by atomic force microscopy (AFM) and dynamic light scattering (DLS) to compare their diameter and aspect ratio. Cotton-CNC suspension was separated for analysis into fractions (as shown in Figure 2.1) to test the importance of three variables: sonication, fractionation (ultracentrifugation), and storage time. Lastly, in order to study the effect of CNC surface chemistry on the mechanical and structural properties, cottonCNCs were modified by sulfation and carboxylation. Please see Chapter 7 for further analysis on these samples.

\subsubsection{Effect of CNC Source on Diameter and Aspect Ratio}

CNCs were visualized using AFM. To prepare samples for AFM, a droplet of the dilute aqueous suspension was placed on a freshly cleaved mica. The sample was air dried and then imaged in a Veeco Dimension Icon using a silicon cantilever, under peak 
force tapping mode. The diameter of the whiskers was measured using section analysis option in Nanoscope 8.10 software.

The diameter of single CNCs was measured using section analysis. As shown in Figure 2.3, in order to reduce the effect of AFM tip broadening, instead of side to side length (horizontal distance), the vertical distance between the substrate and the highest point on the whisker was taken to be the diameter. The diameter reported here is the average of at least 25 measurements.

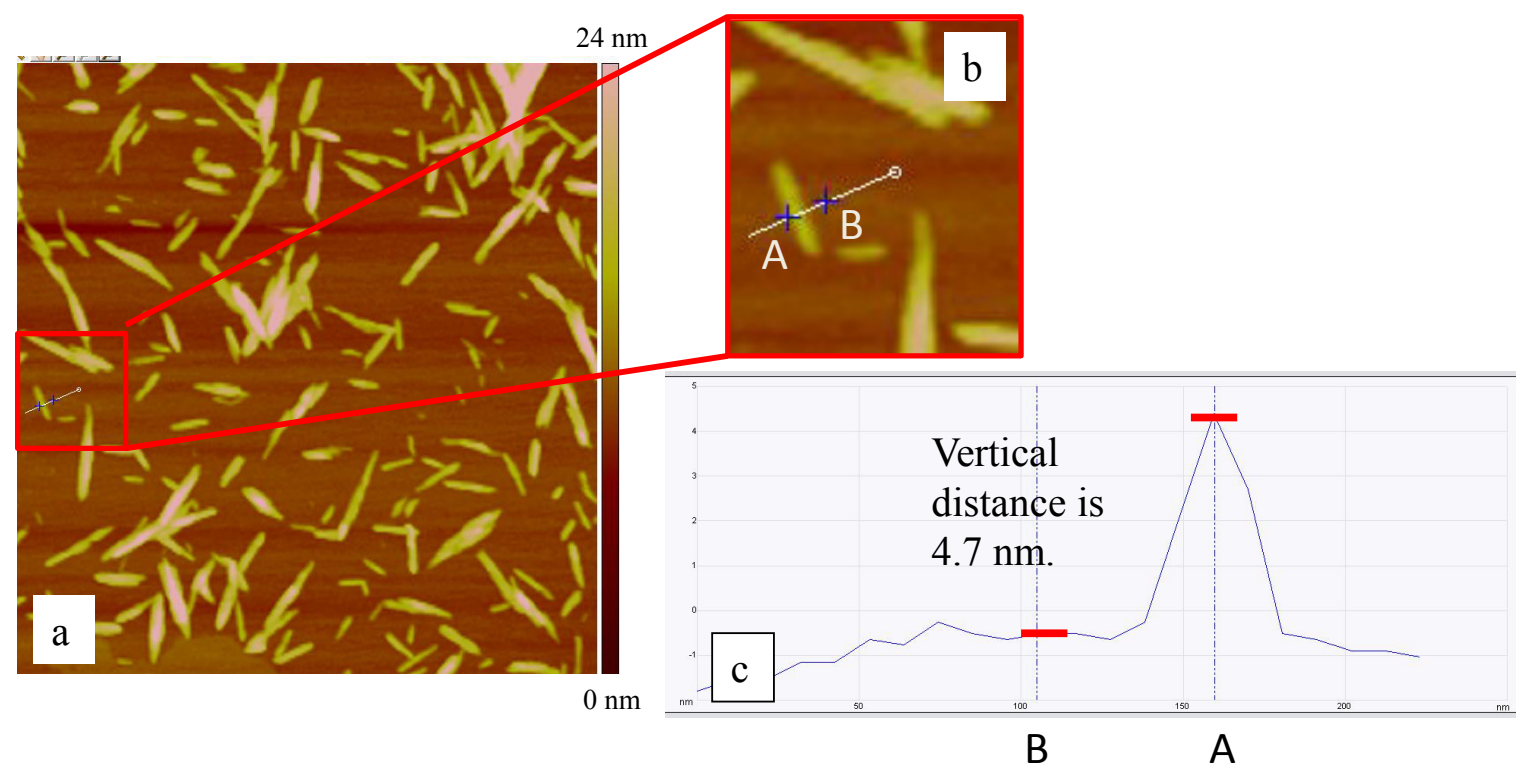

Figure 2.3 Section analysis on the height image to estimate the diameter of CNC. (a) a 2 $\times 2 \mu \mathrm{m}^{2}$ topography image of filter paper-CNCs. (b) Higher magnification image of the region shown in red box in (a). A line is drawn perpendicular to the axis of the crystal. The topography profile along this line is shown in (c). The vertical distance between the substrate and the highest point on $\mathrm{CNC}$ is equal to the diameter.

DLS was used to determine the size and size distribution of CNCs in aqueous suspensions. In DLS, a beam of light passes through a suspension of submicron particles in a liquid. The temporal fluctuations of the scattered light, caused by diffusion motion of particles (Brownian motion) is then measured to obtain the diffusion coefficient $(D)$ and from that the hydrodynamic diameter $\left(d_{h}\right)$ is obtained using Equation 2.1 (Stokes-Einstein equation) (Pecora 1985; Dahneke 1983).

$$
D=\frac{K T}{3 \pi \eta d_{h}}
$$

where $K$ is the Boltzmann constant, $T$ is the temperature, and $\eta$ is the solvent viscosity. DLS was performed in a Beckman Coulter N4 Plus equipped with a $10 \mathrm{~mW} \mathrm{He}-\mathrm{Ne}$ laser, operating for 200 seconds, at $90^{\circ}$ angle and $20^{\circ} \mathrm{C}$. The samples were diluted and sample volumes of $5 \mathrm{ml}$ were used. Two different measurements were performed on each sample, and in each measurement the diameter reported was the average of five tests. 
Figure 2.4 shows AFM images of different CNCs and Table 2.1 presents the results. As will be further discussed in Chapter 6, the diameter and aspect ratio of CNCs after acid hydrolysis greatly depends on the hydrolysis conditions and more importantly their origin. We can see here that CNCs from filter paper and cotton batting have close aspect ratios, as expected because both of these materials are made from cotton. On the other hand MCC- or wood-CNCs have smaller diameter and much higher aspect ratios. As discussed earlier the aspect ratio has great effect on the reinforcing efficiency in nanocomposites. Thus, it can be concluded that among the commonly available resources, MCC results in cellulose nanocrystals which are better candidates for reinforcing applications.
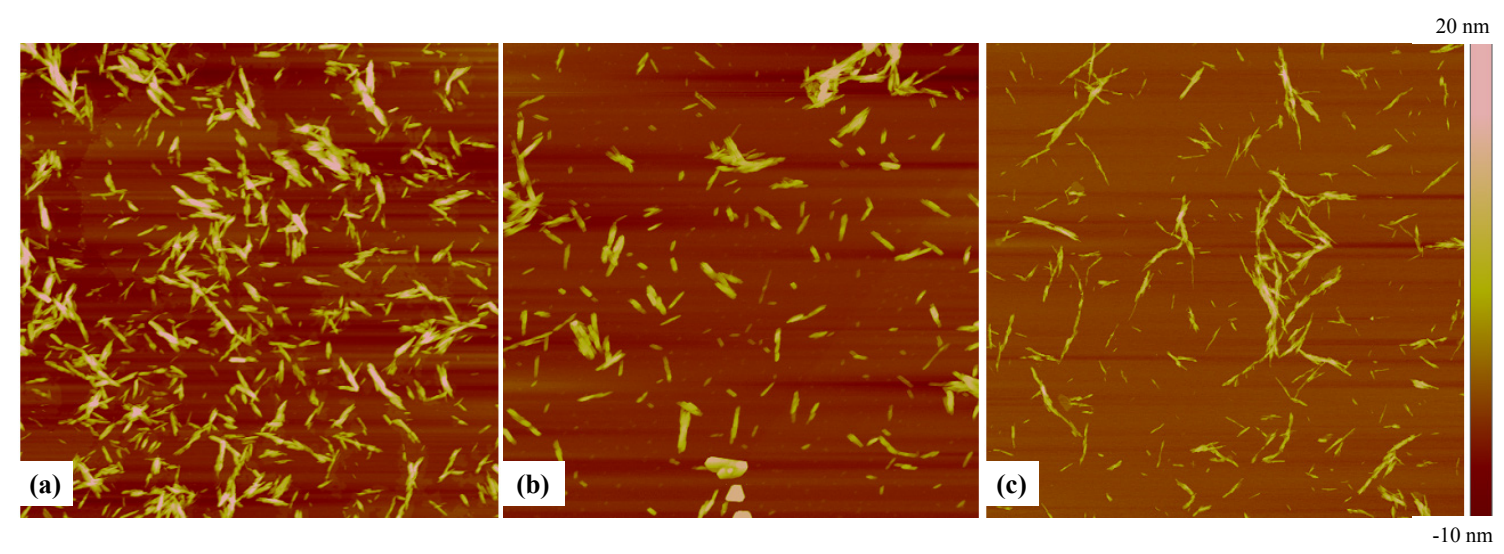

Figure 2.4 The $5 \times 5 \mu m^{2}$ topography images of (a) Filter paper, (b) Cotton batting, and (c) MCC CNCs.

Table 2.1

Comparison of CNCs from different origins

\begin{tabular}{cccc}
\hline \multirow{2}{*}{ CNC origin } & $\begin{array}{c}\text { Length (DLS) } \\
\mathrm{nm}\end{array}$ & $\begin{array}{c}\text { Diameter (AFM) } \\
\mathrm{nm}\end{array}$ & Aspect ratio \\
\hline Filter paper & $140 \pm 63$ & $5.4 \pm 1.0$ & 25.9 \\
Cotton batting & $170 \pm 76$ & $7.6 \pm 1.5$ & 22.3 \\
MCC (wood) & $210 \pm 56$ & $4.4 \pm 1.3$ & 47.7 \\
\hline
\end{tabular}

\subsubsection{Effect of Preparation Steps on CNC Size}

Shelf life: After dialysis, cotton CNCs were stored in a refrigerator at $4{ }^{\circ} \mathrm{C}$ and on a bench top at $24{ }^{\circ} \mathrm{C}$. Based on the hydrodynamic volume $\left(d_{h}\right)$ measurements, the size of the cotton CNCs did not change at either $4{ }^{\circ} \mathrm{C}$ or at $24{ }^{\circ} \mathrm{C}$ after 2 weeks.

Sonication: After CNCs are prepared using acid hydrolysis, they are often sonicated to disperse $\mathrm{CNC}$ aggregates and produce a uniform dispersion of CNCs in water. Here, by keeping the power constant and changing the exposure time, the effect of sonication was studied on cotton CNCs. Sonics Vibra-cell VCX 750 was used to study the effect of sonication on cotton CNCs. Sonication was continued, at constant $60 \%$ power, for 0,2 , 
6, 10 and 14 minutes. During sonication great amount of energy is being transferred to the sample, which can increase the temperature of the solution to an extent that can result in degradation of the crystals. Thus, the water medium was kept in an ice bath, and each experiment was done with $2 \mathrm{~min}$ intervals to avoid heating of the medium.

Samples were sonicated in both glass and plastic vials, as it was suspected that the ions would disperse into the solution from the glass and result in aggregation of the whiskers. Little difference in the results was observed between these two sets of samples. As shown in Table 2.2, sonication time up to 2 minutes decreased the size of the crystals. As the sample is sonicated, the number of aggregates decreases. Consequently, $d_{h}$ and the diameter measured in AFM decrease. After this time, not many aggregates exist. Thus, continuing the process does not make a drastic change in the size distribution. Interestingly, both AFM images and DLS data showed that as sonication time increased from 6 to 10 minutes, the average size also increased. The length decreased again when sonication was continued for 14 minutes.

Table 2.2

Effect of sonication time on CNC length measured by DLS.

\begin{tabular}{cc}
\hline $\begin{array}{c}\text { Sonication Time } \\
\min \end{array}$ & $\begin{array}{c}\text { Length } \\
\mathrm{nm}\end{array}$ \\
\hline 0 & $204 \pm 91$ \\
2 & $130 \pm 59$ \\
6 & $129 \pm 57$ \\
10 & $138 \pm 63$ \\
14 & $131 \pm 60$ \\
\hline
\end{tabular}

Fractionation as a function of CNC size: In order to obtain a mono-dispersed suspension of CNCs and to eliminate any micro-sized particle, ultracentrifugation was used. Suspensions were fractionated using an Eppendorf Centrifuge 5417C at $12000 \mathrm{rpm}$ for 4 minutes. This method has been previously used for fractionation of tunicate cellulose whiskers to decrease the polydispersity of the size of the whiskers (de Souza Lima and Borsali 2002). Figure 2.5 shows the centrifuge vials before and after ultracentrifugation. After the centrifugation was completed, the top (turbid) and bottom (solid) fractions were collected for size analysis.

Table 2.3 summarizes the average particle sizes from DLS before and after the fractionation. Compared to the sample that has not been centrifugated, the samples in solid and turbid portions of ultracentrigugated sample have larger and smaller lengths, respectively Figure 2.6 shows the AFM images of the same cotton samples. The turbid part seems to be more evenly dispersed with respect to size. As opposed to the solid portion, no sign of dirt, amorphous material or micro sized particles was observed in the turbid. Thus, ultracentrifugation is a useful technique for separation of micro sized particles and remaining amorphous regions in water suspension of $\mathrm{CNCs}$ after the acid hydrolysis step. 


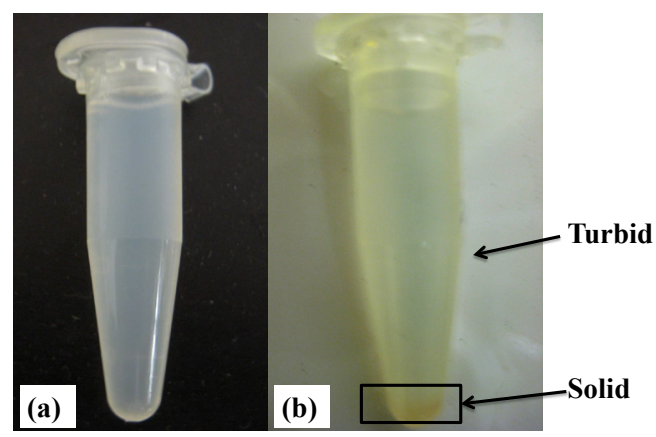

Figure 2.5 Centrifuge vials (a) before and (b) after the ultracentrifugation process, where a small amount of solid is seen at the bottom of the vial.

Table 2.3

Effect of ultracentrifugation on CNC length measured by DLS.

\begin{tabular}{cc}
\hline Sample & $\begin{array}{c}\text { Length } \\
\mathrm{nm}\end{array}$ \\
\hline Before Ultracentrifugation & $204 \pm 91$ \\
Solid & $325 \pm 150$ \\
Turbid & $194 \pm 87$ \\
\hline
\end{tabular}
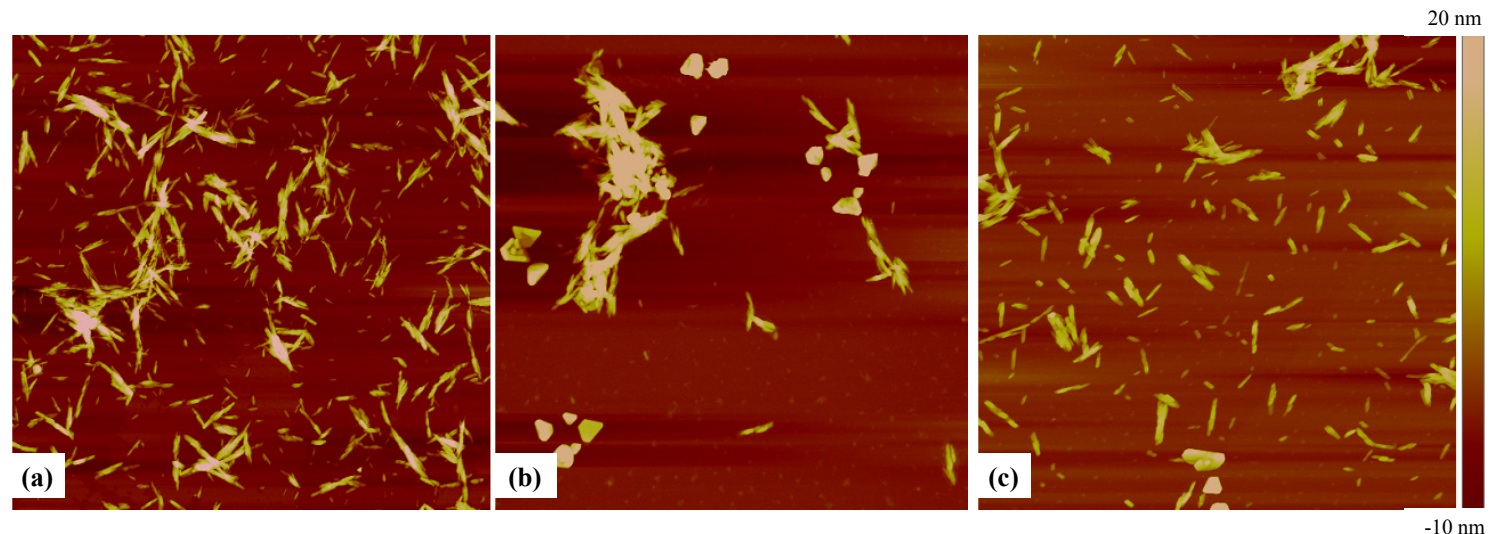

Figure 2.6 The $5 \times 5 \mu \mathrm{m}^{2}$ topography images of cotton CNCs (a) before (b) and (c) after ultracentrifugation. (b) shows the solid and (c) shows the turbid portions, after ultracentrifugation.

\subsection{CNC Surface Modification}

As mentioned earlier, surface properties of CNCs have great effects on their reinforcing efficiency because they define how well the crystals will interact with polymer matrix and their dispersion. The most common method for preparation of CNCs is sulfuric acid hydrolysis which was explained in Section 2.2. During this procedure, small amounts of sulfate ester groups are introduced on the crystal surface (Iwamoto et al. 2009). These crystals will be called sulfated CNCs. 
On the other hand, several reports exist on surface modification of CNCs with a procedure called TEMPO-oxidiation (Saito et al. 2005; Iwamoto et al. 2009). During this procedure, $\mathrm{C} 6$ hydroxyl group on $\mathrm{CNC}$ changes to carboxylate groups. Thus, existence of repulsive forces between these carboxylate groups makes dispersion of these crystals in water achievable by simple mechanical stirring or sonication. It has been shown that during this procedure only the surface properties of the crystals change and the internal structure remain intact (Saito et al. 2005). Here, the procedure used for surface modification of sulfuric acid hydrolyzed CNCs will be explained (Carboxylated CNCs). The structural and mechanical properties of these materials will be compared in Chapter 7.

\subsubsection{Preparation of Carboxylated Cotton CNCs}

After cotton CNCs were prepared by sulfuric acid hydrolysis, a dispersion of $200 \mathrm{~mL}$ of $1 \%$ CNCs was mixed with $2 g$ of $\mathrm{NaBr}$ and $0.2 \mathrm{~g}$ of TEMPO. Then the oxidation reaction was started with the addition of $10 \mathrm{~mL}$ of $\mathrm{NaClO}$ solution and $10 \mathrm{~mL} \mathrm{NaClO}$ solution was repeatedly added after 2 and $4 h$. Overall the reaction took $15 h$. By the addition of $1 \mathrm{~N} \mathrm{NaOH}$ as necessary $\mathrm{pH}$ was maintained at 10 . Next $30 \mathrm{~mL}$ of ethanol was added to terminate the oxidation reaction. In order to remove unreacted reagents, the suspension was ultrafiltered thrice (Ultrasette tangential flow UF device, pore size $=50$ nm, Pall Corp., Ann Arbor, MI). Finally, a calculated amount of $1 \mathrm{M} \mathrm{HCl}$ was added to convert the carboxylates to free acid and he solution was again ultrafiltered until a permeate conductivity of $<5 \mathrm{~S} / \mathrm{cm}$ was obtained. 


\section{Revealing the 3D Internal Structure of Natural Polymer Microcomposites Using X-ray Ultra Microtomography ${ }^{2}$}

\subsection{Introduction}

Amongst various natural reinforcements, much interest has been devoted to the usage of polysaccharides such as chitin, starch and cellulose. Cellulose is the most abundant naturally occurring polymer on earth, and is produced and recycled at a rate of $10^{10}$ tons/year (Hon 1994). It is most widely found in plants (Fleming et al. 2000; Habibi et al. 2008; Siqueira et al. 2009), but some animals (Angles and Dufresne 2000) and bacteria (Grunert and Winter 2002) are also sources of cellulose.

Cellulose consists of both crystalline and amorphous domains. While the amorphous part enables deformation, the crystalline part provides the strength, density, and rigidity (Hamad 2006). The reinforcing efficiency of cellulose depends on the fraction of crystalline domains and their interaction with the domain that they are reinforcing (Lapa et al. 2007). Native cellulose subjected to strong acid hydrolysis readily breaks down into micro- or nano-crystals (Battista 1975).

Despite the above mentioned attractive properties, the lack of control over the dispersion of cellulose crystals in polymer matrices has limited their use in the composite industry (Oksman et al. 2006). Cellulose is a hygroscopic linear polysaccharide of $\beta$-Dglucopyranose units, which are connected by $(1 \rightarrow 4)$-glycosidic bonds. The strong intermolecular hydrogen bonding forces, arising from the hydroxyl groups on the cellulose chains, cause a major fabrication difficulty. The hydrophilic nature of cellulose and the non-polar characteristics of most thermoplastics make it difficult to achieve a sufficient dispersion of this reinforcement in polymer matrices.

Conventional methods for characterization of microstructures in composite materials are generally limited to study of the surface or a localized portion of the composite volume. In addition, they often require tedious sample preparation steps, or they are complex in the sense of data interpretation. These methods include: optical microscopy (OM) (Wang and Sain 2007), scanning electron microscopy (SEM) (Choi and Simonsen 2006), transmission electron microscopy (TEM) (Bodenson and Oksman 2007), atomic force microscopy (AFM) (Matsumura and Glasser 2000), small angle X-ray scattering (Mele et al. 2002) and wide angle X-ray diffraction (Vaia and Liu 2002).

Recently a technique of X-ray ultra microscopy and microtomography (XuM) has been developed, which offers the capability of observing the internal structure of opaque

\footnotetext{
${ }^{2}$ The material contained in this chapter has been previously published in Journal of Microscopy (2011, 243:77-85). See Appendix C for proper documentations.
} 
materials at sub-micron scales. The idea of X-ray projection microscopy originated in the 1950's (Cosslett and Nixon 1951; Nixon 1955), but such systems were developed and made usable only in the last decade (Mayo et al. 2002; Mayo et al. 2003). Tomography has been performed in various media, for example, acoustic waves (Maurer et al. 2006) or neutrons (Dierick et al. 2005) and X-rays. Some other examples of current techniques established for X-ray micro computed tomography are: synchrotron X-ray tomography (with less than one $\mu \mathrm{m}^{3}$ resolution) (Bonse and Bush 1996; Laiarinandrasana et al. 2010), and laboratory desktop microtomography, which can reach down to $5 \mu \mathrm{m}$ spatial resolution (Weiss et al. 2005). The differences between these methods are the X-ray generation source, X-ray source-sample-detector configuration, imaging resolution and acquisition time. The resolution of XuM will be discussed in following sections of this chapter.

X-ray computed tomography has many interesting applications in different research areas. In the field of polymers, for instance, it has been used to study the void volume fraction in necking region of tensile test specimens (Laiarinandrasana et al. 2010). XuM is well suited to examine the quality of dispersion of reinforcements in polymer composites. Other attractive advantages over conventional microscopy techniques include easier sample preparation, the nondestructive nature of analysis, and the ability to obtain large scale statistical information of the internal structure.

Given the difficulties associated with quantification of cellulose dispersion in polymer matrices, in this study, XuM was used to study the internal structure of partially hydrolyzed cellulose microcrystals (CMC) dispersed in polycaprolactone (PCL). CMC and PCL have low chemical compatibility and since the surface of PCL or CMC were not chemically modified, thus a homogeneous dispersion and mechanical reinforcement were not expected. The main focus of this work is to demonstrate the applicability of XuM to reveal the 3D views of CMC clustering and tangling within PCL matrix. A direct correlation between the bulk mechanical properties and the XuM data was also made. The results demonstrate the feasibility of this technique for dispersion quality analysis of other natural polymer composites.

\subsection{Materials and Methods}

Cellulose composite film preparation: PCL was selected as the matrix to prepare composite films. PCL is a linear hydrophobic polyester, which is synthetically produced and is biodegradable. Commercially available CMC, produced by sulfuric acid treatment of bleached Kraft wood fibers followed by washing and drying, was partially hydrolyzed and used at $0,10,30$ and $50 \mathrm{wt} . \%$. Hereafter, these composites are named PCL 0 , PCL 10, PCL 30 and PCL 50 respectively. Sulfuric acid hydrolysis of the CMCs was done under moderate conditions. The hydrolysis was insufficient to give nanocrystals but did begin the defibrillation process. In summary, $5.6 \mathrm{~g}$ commercially available CMC was placed in $64 \% \mathrm{H}_{2} \mathrm{SO}_{4}$ solution for $1 h$ at $45^{\circ} \mathrm{C}$ with medium stirring. The cellulose to acid ratio was 1:10. After 45 minutes, de-ionized water was added to the solution to stop the reaction. Afterwards, centrifugation at $4000 \mathrm{rpm}$ was used to wash the solids until a 
neutral $\mathrm{pH}$ was obtained (IEC DPR-6000 centrifuge). Next, the solids were placed in dialysis bags and washed against de-ionized water for several days.

Evenly dispersion of cellulose in PCL matrix is critical for improvement of mechanical properties. Because cellulose is polar and PCL is non-polar, this is only possible if the surface polarity of CMC is changed (Siqueira et al. 2009) or if PCL is directly grafted to CMC (Habibi and Dufresne 2008). The goal here was not to improve the mechanical properties, but to characterize the distribution of CMC in PCL. Thus, composite films were prepared by simple mixing and without any chemical modification of CMC or PCL.

Partially hydrolyzed $\mathrm{CMCs}$ were dispersed in $\mathrm{CHCl}_{3}$ and sonicated for $0.5 \mathrm{~h}$. Separately, PCL was dissolved in $\mathrm{CHCl}_{3}$ and then mixed with the sonicated crystals, as they were vigorously stirred with a mechanical stirrer. The stirring was continued for two hours after the addition was completed. Afterwards, the solvent was removed under reduced pressure at $60{ }^{\circ} \mathrm{C}$, and the combination was transferred into molds and heated at $140{ }^{\circ} \mathrm{C}$ under $20 \mathrm{MPa}$ pressure, for 10 minutes.

Mechanical testing: Mechanical characterization of the polymer composites was carried out using a MTS universal testing machine with a $4.5 \mathrm{kN}$ load cell. All samples were cut in equal rectangular shapes. The gage length dimensions of the samples were 0.7 $\times 4 \times 6 \mathrm{~mm}^{3}$, and at least three tests were performed with each composition to work around any experimental scatter. Load was applied by moving the cross head with 0.127 $\mathrm{cm} / \mathrm{min}$ rate. Load-displacement curves were obtained and converted to engineering stress-strain curves using the initial length and area of each sample. Reported ultimate tensile strength (UTS) values are average values of at least three tests (Table 3.1).

Table 3.1

The UTS values in PCL microcomposites

CMC content (wt. \%)

UTS (MPa)

\begin{tabular}{cc} 
(wt. $\%)$ & $17.48 \pm 0.33$ \\
0 & $14.55 \pm 0.16$ \\
10 & $10.3 \pm 0.21$ \\
30 & $7.55 \pm 2.76$ \\
50 & \\
\hline
\end{tabular}

Morphological analysis: XuM work was carried out at Gatan Inc, Pleasanton, CA. The XuM setup was installed in a Hitachi S-3400 N SEM. The main components, shown in Figure 3.1a, are a rotation stage, charge-coupled detector (CCD) X-ray camera and a target module, which positions up to 5 metal targets and allows operating X-ray microscopy inside a SEM. In this method, the X-ray images are obtained by focusing the electron beam on a metal target, and passing the generated X-rays through the sample. Adopting the point projection geometry principles, 2D X-ray images can be recorded. In order to obtain a $3 \mathrm{D}$ visualization, $2 \mathrm{D}$ images are taken as the sample rotates in small 
angle increments, and then the projections are computationally combined (see next section).

In order to acquire 2D images, a very small X-ray source is created on a thin metal target by focusing the electron beam (Figure 3.1b). A sample is then placed between the source and the CCD. This results in a simple point projection geometry (Cosslett and Nixon 1960) where X-rays pass through the specimen and create a magnified image on the detector. Usually the source to sample distance $\left(R_{1}\right)$ is much smaller than the source to detector distance $\left(R_{2}\right)$ and this enables magnifications $\left(M=R_{2} / R_{1}\right)$ up to $2000 \mathrm{x}$. Depending on the electron beam energy and the target material, X-ray energy may vary between 4 and $12 \mathrm{KeV}$. Imaging resolution greatly depends on the X-ray source size (Goldstein et al. 2003), and can be as small as $200 \mathrm{~nm}$ in 2D and $1 \mu \mathrm{m}$ in 3D.

Because of the small source size and $R_{l}$, in-line phase contrast is obtained on the image plane, in addition to absorption contrast, as a result of near-field Fresnel diffraction (Mayo et al. 2003; Myers et al. 2008). Phase contrast enhances the visibility of boundaries, voids and edges and is critical for weakly- or non-absorbing samples, such as polymers. 


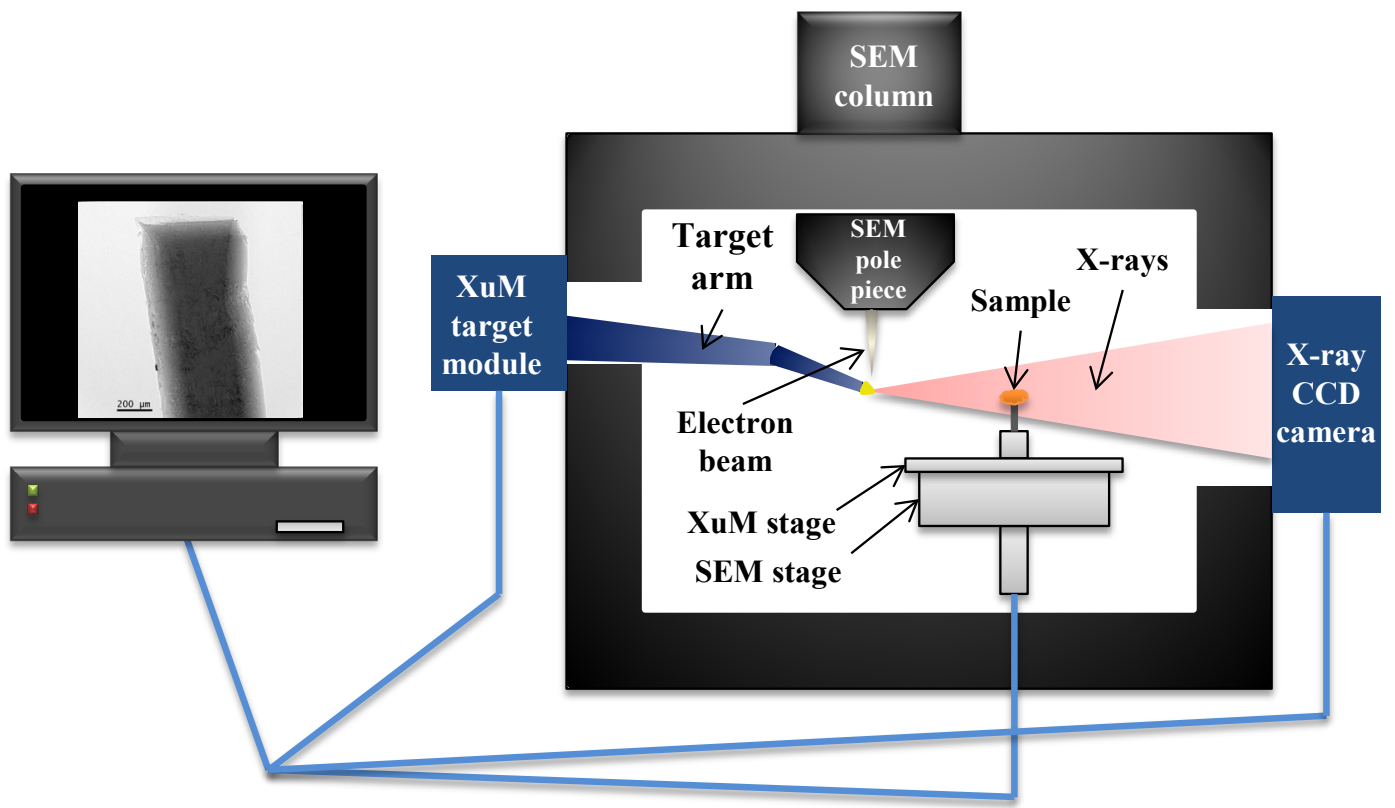

(a)

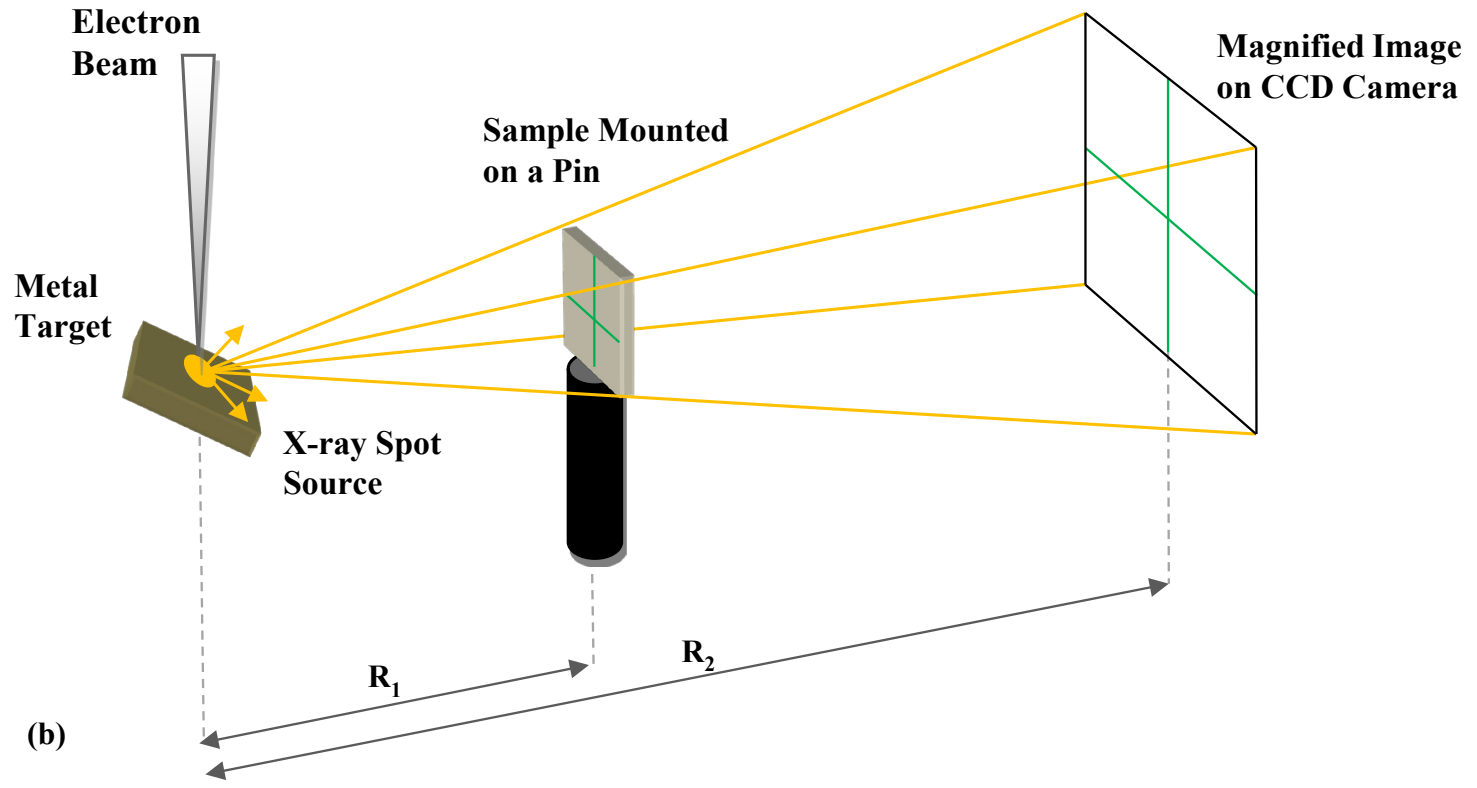

Figure 3.1 (a) Main components of the XuM inside SEM, in order to generate X-rays, a metal target is positioned under the electron beam using the target module. X-rays pass through the sample and create the 2D image on the CCD camera. (b) Point projection geometry in XuM; the magnification $\left(M=R_{2} / R_{1}\right)$ can be changed by moving the sample towards or away from the X-ray source. 


\subsection{Selection of Target Material and 3D Reconstruction}

The resolution and energy of X-rays are directly related to the choice of target material, and thus a systematic study was performed to select an appropriate target. For this purpose, a graph of accelerating voltage versus intensity of eleven metal targets, along with the imaging resolution at each point was created (Figure 3.2). To generate this graph, NIST/NIH Desktop Spectrum Analyzer (DTSA-II) was used to create X-ray spectra (intensity versus accelerating voltage) generated by the targets under various accelerating voltages. The highest peaks on these spectra were then used as intensities. These intensities were normalized relative to the intensity of X-rays generated by $\mathrm{W}$ at 30 $\mathrm{KeV}$, and are shown on the vertical axis.

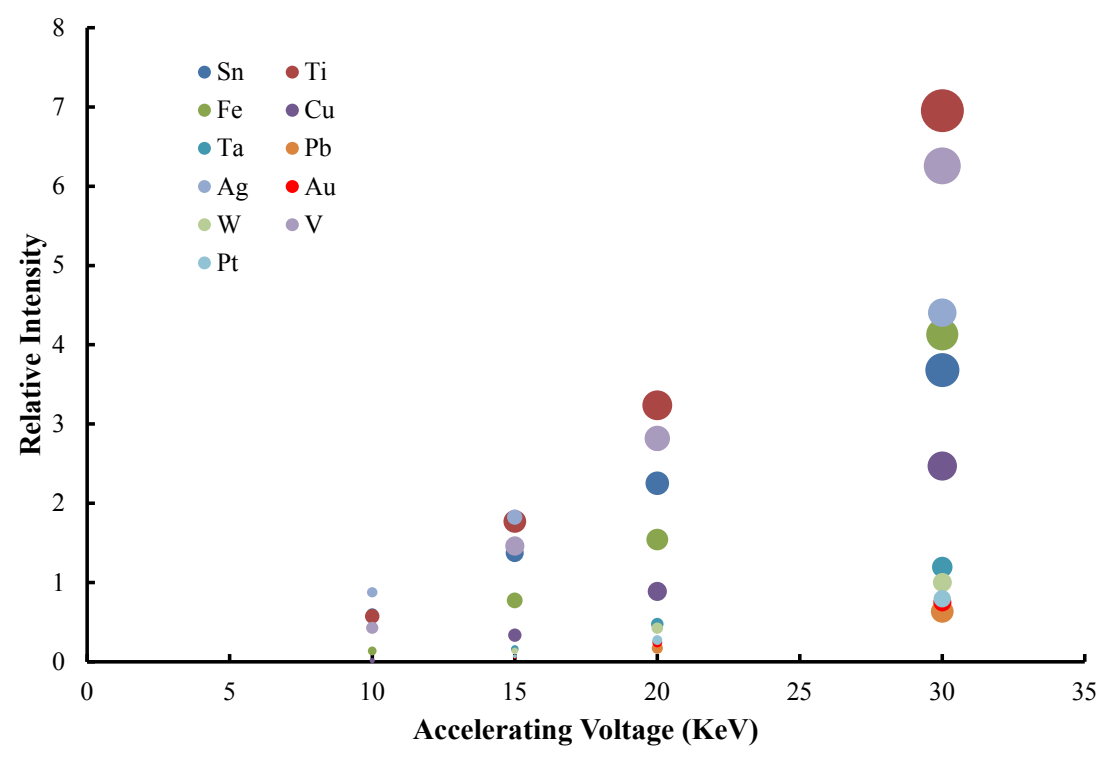

Figure 3.2 Relative intensity and resolution of XuM metal targets. The diameter of the circles on the graph represents the imaging resolution in micrometers. Intensities were reported relative to intensity of $\mathrm{W}$ at $30 \mathrm{KeV}$.

Anderson-Hasler X-ray range $(R)$ was used as the imaging resolution (X-ray source size) for each metal (Goldstein et al. 2003). Resolution is shown by the diameter of the circles on the graph and depends on the incident energy $\left(E_{c}\right)$ and the material properties of the target $\left(\rho=\right.$ density, $E_{0}=$ critical excitation energy):

$$
R=\frac{0.064}{\rho}\left(E_{0}^{1.68}-E_{c}^{1.68}\right)
$$

The choice of target material and the accelerating voltage of the SEM are two major parameters that affect the resolution (shown as the size of circles with diameters of less than $2 \mu \mathrm{m}$ ), and the intensity of X-rays, and in-turn the quality of XuM data. It can be seen from Figure 3.2 that higher accelerating voltage results in higher intensities but reduction in maximum resolution at the same time. For instance, in the case of V, when 
the accelerating voltage is increased from 10 to $30 \mathrm{KeV}$, the relative intensity increases almost 14 times but the theoretical resolution changes from 0.16 to 1.50 microns.

Next, four metal targets namely $\mathrm{Ag}, \mathrm{V}, \mathrm{W}$ and $\mathrm{Au}$ were selected to experimentally examine their ability to enhance the contrast of specific features in a sample. Samples were mounted on Al pins and coated with $20 \mathrm{~nm}$ Pt in a Gatan precision etching coating system (PECS). Then 2D X-ray images were acquired using these metal targets and various accelerating voltages $(10$ to $30 \mathrm{keV})$. The best quality images with the highest contrast and flux (signal to noise ratio, $S / N$ ), were obtained using V foil target with an accelerating voltage of $30 \mathrm{keV}$, and beam current of $>200 \mathrm{nA}$ (Figure 3.3).

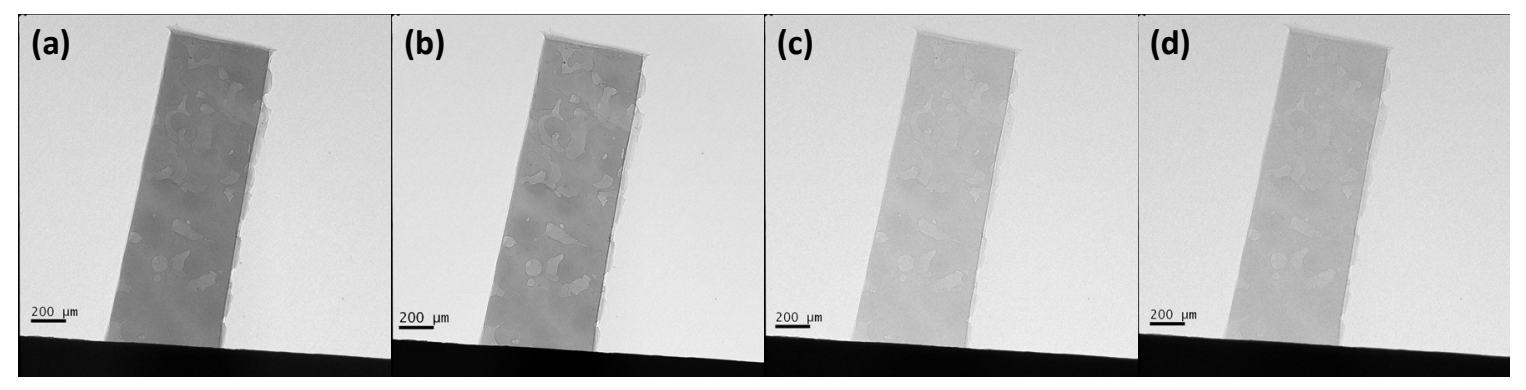

Figure 3.3 2D XuM images of PCL samples obtained under (a) Ag, (b) V, (c) W, (d) Au metal targets. The accelerating voltage was fixed at $30 \mathrm{KeV}$. The choice of $\mathrm{V}$ target was preferred because of its optimal contrast and flux.

In order to produce a 3D reconstruction for each sample, a tomographic dataset containing one hundred and ninety $2 \mathrm{D}$ projections with one-degree increments between each projection was obtained. In order to acquire the adequate signal to noise ratio $(S / N$ $>2$ ), each projection consisted of at least three frames, with minimum of 10 seconds exposure time. These images are then back projected using a cone-beam back projection algorithm (Feldkamp et al. 1984) to create a 3D image of the volume.

\subsection{Results and Discussion}

Figure 3.4 shows the typical engineering stress-strain curves obtained for the microcomposite samples, while Table 3.1 summarizes the tensile test results as ultimate tensile stress (UTS). Addition of CMC to PCL degraded the UTS. As the amount of CMC increased from 0 to $50 \mathrm{wt} \%$ the UTS decreased from 17.48 to $5.5 \mathrm{MPa}$ (=68\% decrease). No major changes were observed in the elastic modulus, but as the amount of CMC was increased, the yield stress appeared to decrease in PCL composites.

An important observation is that the plateau of stress-strain curves almost disappeared in PCL 30 and PCL 50 yet this range was the highest in PCL 10. Due to lack of good adhesion between polar CMC and non-polar PCL, at high concentration of CMCs (30 and $50 w t . \%$ ), debonding at the CMC and PCL interface is the governing mechanism, while at $10 w t . \%$, the CMCs underwent reorientation along with sliding of polymer chains, which accounted for the large deformation plateau in the PCL10 samples. 


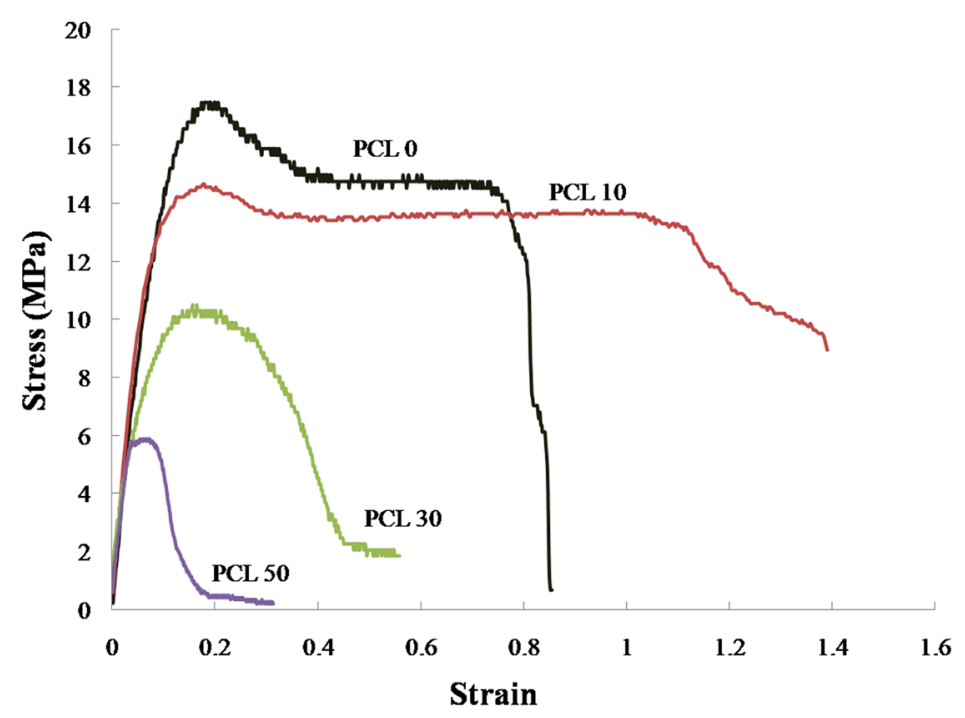

Figure 3.4 Engineering stress-strain curves for PCL composites: black: PCL 0, red: PCL 10, green: PCL 30, and purple: PCL 50

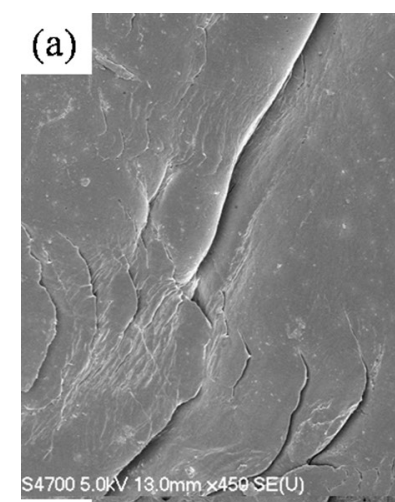

(c)
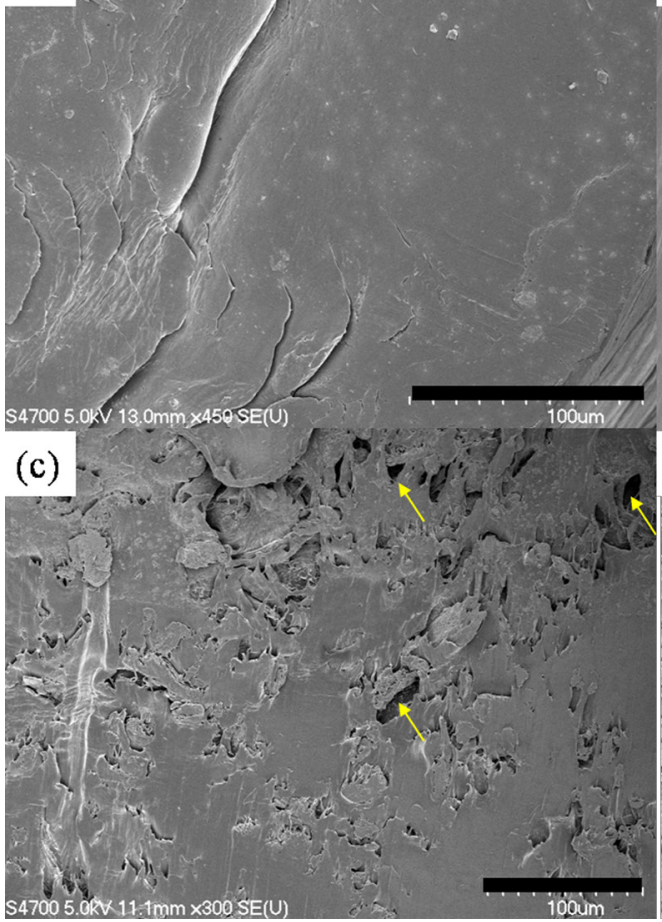

(b)

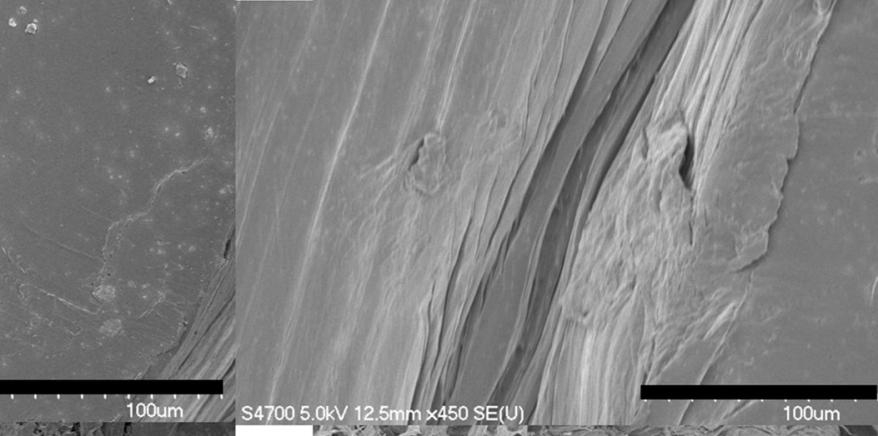

(d)

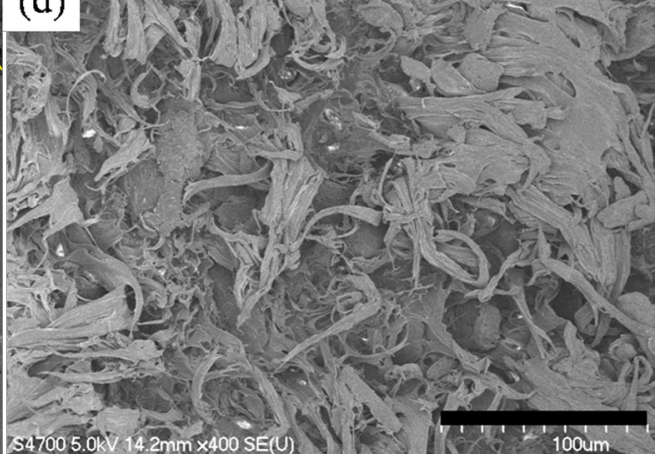

Figure 3.5 SEM images from the fracture surface of CMC-PCL composites: a) PCL 0, b) PCL 10, c) PCL 30, d) PCL 50; Arrows in (c) show voids that were created as a result of $\mathrm{CMC}$ being pulled out during the tensile test. (Scale bars are $100 \mu \mathrm{m}$ )

Fractography of the PCL composites was carried out using a Hitachi S-4700 field emission SEM operating under a voltage of $3 \mathrm{KeV}$ (Figure 3.5). Although only one SEM 
image is shown here, for each composition, at least four fracture surfaces were studied. Relatively smooth surfaces were observed in PCL 0 and PCL 10 samples (Figure 3.5 a and $b$ ). As the amount of $\mathrm{CMC}$ increased, the fracture surface became rougher and more voids and ridges could be detected (Figure $3.5 \mathrm{c}$ and d). PCL with $30 \mathrm{wt} \% \mathrm{CMC}$ appeared to have many voids, which may be areas where CMCs debonded during tensile experiments. The fracture surface of PCL 50 showed the roughest surface.

The correlation of fractography data and stress-strain curves revealed two competing mechanisms during the deformation of CMC-polymer composites. The reduction of UTS in all the $\mathrm{CMC}$-samples indicates that the bonding between $\mathrm{CMCs}$ and polymer matrix was weak. However, in PCL 10, CMCs have successfully rendered the void coalescence and therefore allowed the movement of polymer molecular chains up to higher strains. At $30 \%$ and $50 \%$ CMC content, the void population increases due to the higher number of debonding incidents, and therefore void-assisted fracture dominated the deformation of composites.

The 2D X-ray images of the samples are shown in Figure 3.6 (similar to fractography, for each composition, multiple samples were prepared and studied in the XuM). X-ray images show the dispersion of CMC through the specimens. Good contrast on CMC distribution was obtained for specimens, because the difference between X-ray absorption of PCL and CMC is high enough and as a result there is difference in the intensity of regions corresponding to PCL or CMC in the images. In addition to absorption contrast, phase contrast accentuated both the interphase regions and the cracks.

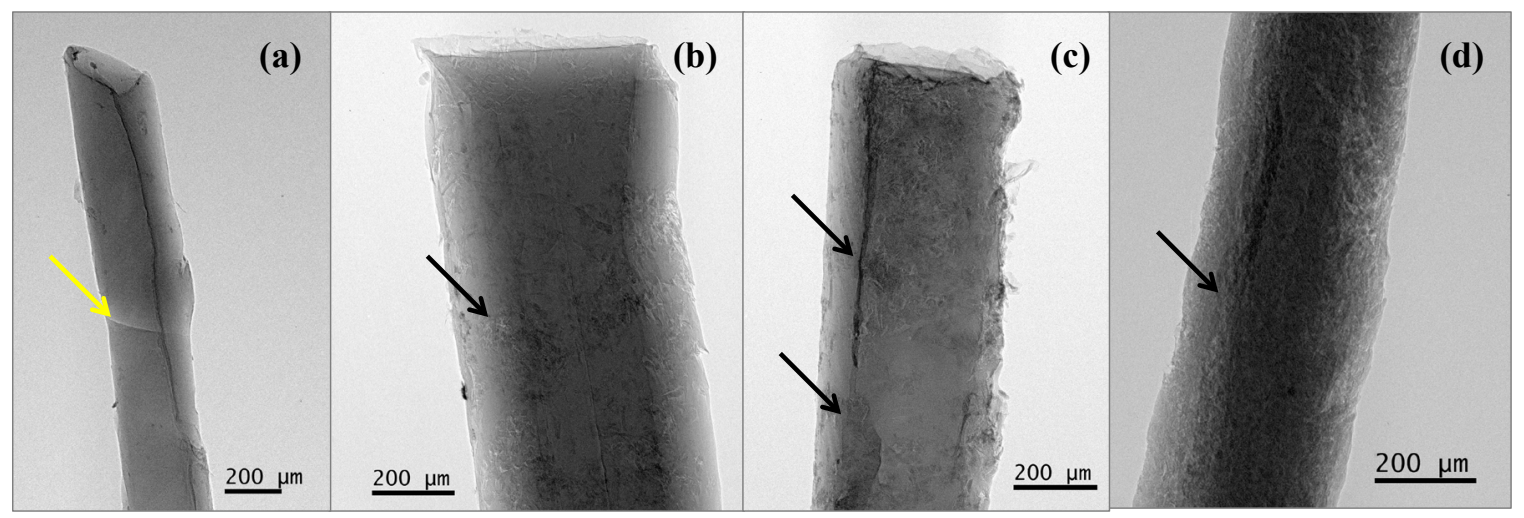

Figure 3.6 2D XuM images of PCL composites: (a) PCL 0, (b) PCL 10, (c) PCL 30, and (d) PCL 50. Some of the CMCs are shown by black arrows on the images. The yellow arrow in (a) shows a crack in the sample, which was created during sample preparation (phase contrast). (Scale bars are $200 \mu \mathrm{m}$ )

Figure 3.7 shows the $3 \mathrm{D}$ reconstruction of a composite sample. CMC appeared as higher density regions in the back projections. After reconstruction, any horizontal or vertical slice through the sample can be studied (Figure $3.7 \mathrm{a}$ and b). These planes can be simultaneously visualized with the 3D volume as shown in Figure $3.7 \mathrm{c}$. 

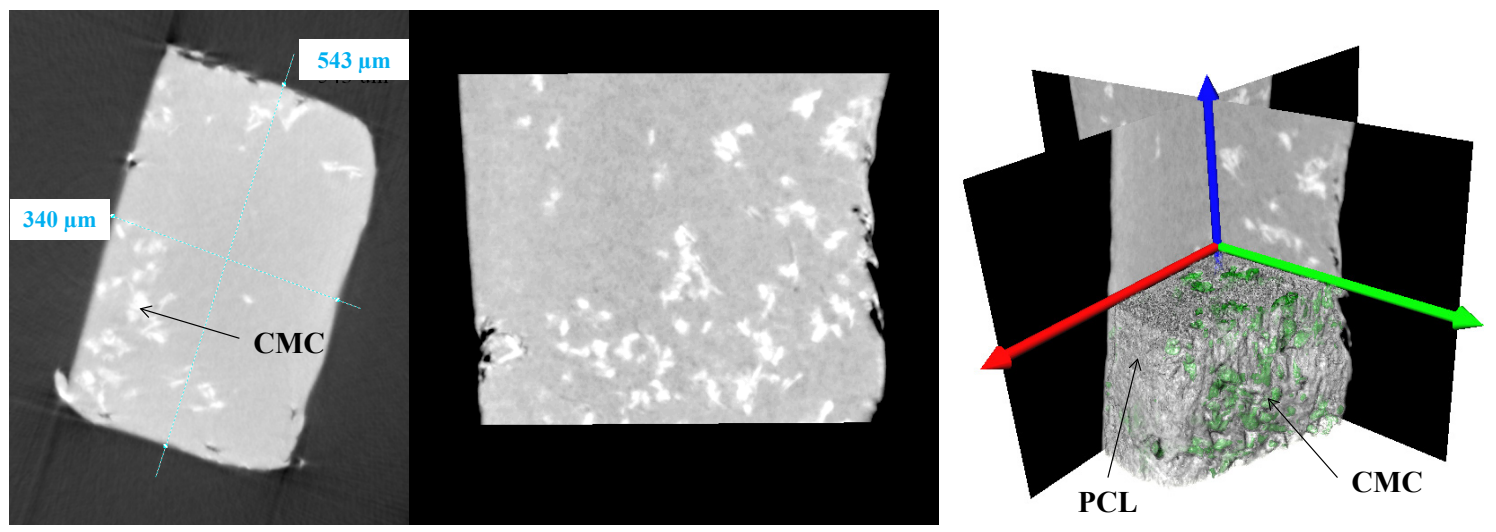

Figure 3.7 3D reconstruction of a PCL sample filled with $10 w t . \% \mathrm{CMC}$. CMC can be recognized as bright regions. (a) Horizontal slice of 340 by $543 \mu \mathrm{m}^{2}$ through the sample. (b) Vertical plane through the specimen. (c) 3D volume is shown along with two slices through the sample. PCL and CMC are shown as gray and green zones.

Figure 3.8 depicts the PCL specimens in 3D. PCL and CMC are shown in gray and green colors respectively. As can be seen, some regions of the reinforced samples have clusters of CMCs while other regions are purely PCL. This correlates directly to degradation of UTS as the CMC content is increased. Due to the weak interface bonding, these clusters act as stress concentration points, where debonding and fracture start. Microfractures and voids propagate as the stress increases and they ultimately result in the failure of the material.

(a)

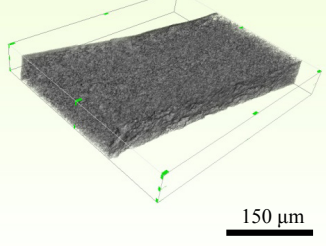

(b)

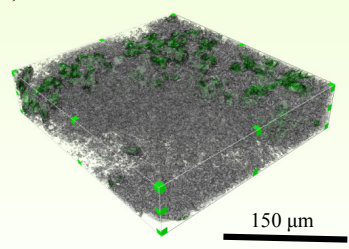

(c)

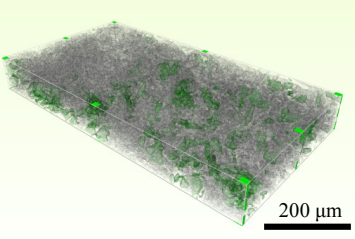

(d)

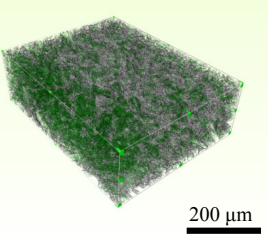

Figure 3.8 3D visualization of PCL samples filled with a) 0 b) 10 c) 30 and d) $50 w t . \%$ CMCs. PCL and CMCs are shown in gray and green respectively.

To study the size and distribution of CMCs, statistical analyses on the cluster diameter, aspect ratio and orientation distributions were obtained on a single vertical plane through the sample (Figure 3.9). In this work, the cluster diameter was assumed to be equal to an equivalent cluster diameter $\left(C D=2 \sqrt{\frac{A}{\pi}}\right)$ calculated from the area $(A)$ of particles (Jennings and Parslow 1988). 

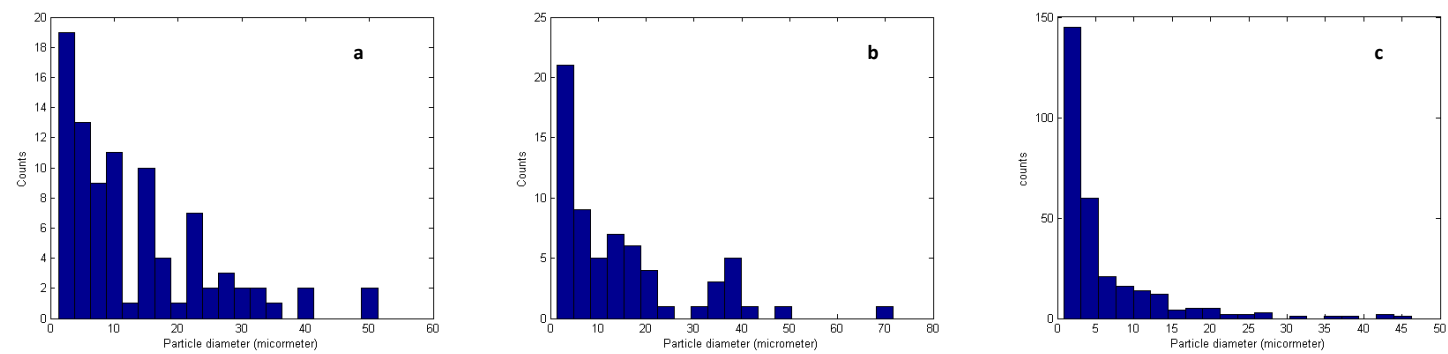

Figure 3.9 Histograms show the distribution of the CMC-cluster diameters in PCL composites: a) PCL 10 b) PCL 30 c) PCL 50. As opposed to PCL 10 and PCL 30, the number of small sized ( $<5$ micrometers) clusters is higher in PCL 50.

In literature, there are numerous studies on the effect of reinforcement geometry and concentration on the mechanical properties of polymer composites (Choi and Simonsen 2006; Bodenson and Oksman 2007). The 3D characterization by XuM enables one to access such statistical information on the geometrical properties of reinforcements. Here, the average diameter of $\mathrm{CMC}$ clusters, their population and their aspect ratios (the ratio of the size of the major axis to the minor axis in each particle) are summarized in Table 3.2 .

Table 3.2

Statistical parameters of CMC-polymer composites

\begin{tabular}{cccc}
\hline $\begin{array}{c}\text { CMC } \\
\text { content } \\
(\boldsymbol{w} \boldsymbol{t} \%)\end{array}$ & $\begin{array}{c}\text { Average } \\
\text { cluster } \\
\text { diameter } \\
(\boldsymbol{\mu m})\end{array}$ & $\begin{array}{c}\text { Average } \\
\text { aspect } \\
\text { ratio }\end{array}$ & $\begin{array}{c}\text { Population } \\
\text { of clusters }\end{array}$ \\
\hline 10 & 13.1 & 1.8 & 89 \\
30 & 14.7 & 5.8 & 65 \\
50 & 5.8 & 1.8 & 295 \\
\hline
\end{tabular}

The high population of small clusters and lack of good adhesion between CMC and PCL resulted in the lowest value of UTS in PCL 50 (Table 3.1) because the void formation rapidly increased and became the dominant failure mechanism. This observation is in good agreement with fractography analysis, where the fracture surface was much rougher in PCL 50 than pure other samples (Figure 3.5d).

Another observation is that the size and population of clusters are not considerably different in PCL 30 and PCL 10. However, the clusters in PCL 30 had a much higher average aspect ratio as compared to the ones in PCL 10. Usually higher aspect ratio reinforcements result in better stress transfer and improvement of mechanical properties (Azizi Samir et al. 2005; Hamad 2006). In the current study, since CMCs did not make good adhesion with the polymer matrix, these high aspect-ratio particles acted as high stress-concentration areas, and resulted in failure at much lower loads. Lastly, it should be mentioned that this statistical analysis was performed only on one vertical section 
from each sample. For more accurate analyses, it is necessary to employ stereology techniques (Russ and Dehoff 2000) to extract 3D information from 2D images. As such, multiple cross sectional images need to be analyzed to represent a statistically valid conclusion.

\subsection{Conclusions}

SEM imaging and XuM were used to study the dispersion quality of reinforcements in polymer matrices, and their relationship with the overall mechanical properties of the composites. XuM images provided complementary information about the spatial distribution of CMC in PCL composites. This information can be used for better understanding of the behavior of variety of composite materials and further improvement of their qualities.

The spatial resolution of the reconstructed 3D volumes was sufficient to eliminate all suspicions about the quality of dispersion of the CMCs within the whole volume. Vertical slices through these volumes were used for statistical analysis on the size, shape and orientation of the CMCs.

The correlation of fractography and XuM data with the stress-strain curves revealed two mechanisms during the deformation of CMC-polymer composites. The size, shape and distribution of $\mathrm{CMC}$ clusters, had high effects on the void formation and crack propagation rates in the materials and weak bonding between CMC and PCL resulted in reduction of UTS in all composite samples. On the other hand, $10 w t . \% \mathrm{CMC}$ improved the flow of polymer molecules under stress and improved the ductility and toughness of PCL.

\subsection{Acknowledgments}

We would like to thank Gatan Inc. (Pleasanton, CA) for providing the opportunity to use the X-ray Ultra Microscope (XuM). Reza S. Yassar acknowledges Michigan Technological University to provide seed funds to conduct this work. 


\section{Elastic Properties of Thin Poly(vinyl alcohol)-Cellulose Nanocrystal Membranes $^{3}$}

\subsection{Introduction}

The mechanical properties of polymer films have been thoroughly investigated using tensile tests (Azizi Samir et al. 2004; Cao et al. 2007; Wang et al. 2006) and nanoindentation in the atomic force microscope (AFM) (VanLandingham et al. 1997; Winter and Houston 1998; Gao and Mader 2002; Tranchida et al. 2006). The high depth and force resolution capabilities of AFM (Capella and Dietler 1999), which are not achievable by any other tool make it a strong apparatus for nanoindentation characterization of thin films (Chizhik et al. 1998, Tranchida et al. 2006). One of the most important advantages of using AFM (vertical resolution $0.1 \mathrm{~nm}$, load resolution 5 $n N$ ) instead of conventional nanoindenters is the versatility of applied load ranges, by using cantilevers with different spring constants (Capella and Dietler 1999). Specifically in the case of thin films, in order to avoid any effects of the substrate, it is vital that small loads and shallow indentations are applied to the surface (Downing et al. 2000; Gao and Mader 2002). Also, in the combined AFM-Nanoindentation system, it possible to image the indentation area just after the load is removed. This reduces uncertainties in calculations of the contact area (Karapanagiotis et al. 2002).

Poly(vinyl alcohol) (PVA) is a versatile polymer with applications as a membrane material for chemical separation, manufacturing materials for artificial human tissue, and controlled drug release hydrogels (Finch 1992; Bourke et al. 2003; Schmedlen et al. 2002; Wan et al. 2002). Because of its hydrophilicity, PVA is not stable in water unless it is cross linked, and this can limit its potential applications in places where water insolubility or resistance to swelling is needed (Finch 1992). Cross linking of PVA can be accomplished by using either heat treatment (Kumeta et al. 2003) or a cross linking agent such as poly(acrylic acid) (PAA) (Arndt et al. 1999). In a previous report, we prepared PVA-PAA-cellulose nanocrystal (CNC) membranes and characterized their bulk mechanical, thermal, and barrier properties (Paralikar et al. 2008). It was shown that an ester linkage was formed between the carboxyl group in PAA and hydroxyl group in CNC and PVA, which resulted in a highly networked structure with improved moisture properties (Paralikar et al. 2008).

CNCs are high elastic modulus nanocrystals (Sturcova et al. 2005), which are prepared by acid hydrolysis of cellulose fibers from different sources (Bondeson et al. 2006; Hubbe et al. 2008). CNCs recently have attracted great attention as nano-reinforcements in polymers because of their renewable nature, biodegradability, biocompatibility, and

3 The material contained in this chapter has been accepted for publication in Nanotechnology. See Appendix C for proper documentation. 
low cost (Azizi Samir et al. 2004; Choi and Simonsen 2006; Wang et al. 2006; Bodenson and Oksman 2007; Cao et al. 2007).

To the authors' knowledge not many reports exist on the systematic comparison of the surface nanomechanics with bulk mechanical properties in CNC-reinforced polymeric thin films (Miyake et al. 2006). The purpose of this study was to compare the surface nanomechanical properties with those at the macro-scale in PVA-PAA-CNC composites. It was shown that the elastic moduli obtained in AFM were higher than macro-scale tensile elastic moduli. We found that $\mathrm{CNC}$ and PAA improved the elastic modulus of PVA. Interestingly, the proper selections of experimental parameters such as loading rate and maximum applied load have a great impact on the nanomechanical measurements.

\subsection{Materials and Methods}

Cotton (Whatman \#1 filter paper, Clifton, NJ) was partially hydrolyzed to prepare CNCs. For this purpose, filter paper was ground and stirred with $65 \% \mathrm{H}_{2} \mathrm{SO}_{4}(v / v)$ for 50 $\mathrm{min}$, at $45^{\circ} \mathrm{C}$ (cotton to acid ratio, $1: 10 \mathrm{~g} / \mathrm{mL}$ ). Then the mixture was centrifuged 5 times, and it was ultrasonicated (Branson Sonifier, Danbury, CT) for $15 \mathrm{~min}$, in order to evenly disperse the CNCs in water. The next step was ultra filtration until the conductivity was $<10 \mu \mathrm{S} / \mathrm{cm}$ (Ultrasette tangential flow UF device, pore size $=50 \mathrm{~nm}$, Pall Corp., Ann Harbor, MI), to remove remaining salts. Finally an aqueous suspension of $1 \% \mathrm{CNC}$ was obtained by concentrating the dispersion of CNCs in a Rotavaporizer R110 (Buchi, Flawil, Switzerland). For further details regarding the materials and sample preparation please see our method in Paralikar et al. (2008).

Poly (vinyl alcohol), (99+\% hydrolyzed, $\left.\mathrm{M}_{\mathrm{w}}=89000-98000\right)$ was obtained from Sigma-Aldrich Inc. (St Louis, MO). Poly (acrylic acid), $\left(\mathrm{M}_{\mathrm{w}}=2000\right)$ was obtained from Aldrich Chemical company Inc. (Milwaukee, WI). PVA and PAA were separately dissolved in DI water and stirred in an oil bath for $30 \mathrm{~min}$, at $85^{\circ} \mathrm{C}$. Calculated volumes of component solutions and $\mathrm{CNC}$ dispersions were then blended and sonicated for $25 \mathrm{~min}$ to disperse any remaining aggregates. Finally, mixtures were cast in a flat-bottomed plastic dish and air dried for $48 \mathrm{~h}$. CNC content was varied between 0 to $20 \mathrm{wt} \% \%$ and the PAA content was varied from 0 to $10 w t \%$.

A universal testing machine (Sintech 1/G, MTS, Cary NC) was used for tensile testing. Using a cutter, the samples were cut into dog-bone shapes. Each experiment was repeated three times (cross head speed of $1 \mathrm{~mm} / \mathrm{min}$ with $20 \mathrm{~mm}$ initial spam). Tensile modulus was calculated as the slope of the linear part of the curves.

\subsection{Nanoindentation in AFM}

A diamond nanoindenter tip, mounted on a stainless steel cantilever was used for imaging and nanoindentation experiments in a Dimension $\mathbb{C}$ Icon $\mathbb{C}$ AFM (Bruker Corporation, CA, USA). This makes it possible to find the surface of interest by imaging, to perform the nanoindentation, and lastly to image the indentation imprint after the test is complete. AFM images were acquired using peak-force tapping mode (PF-TM), which is a new mode developed by Bruker Corporation (Pittenger et al. 2009). In this technique, 
the cantilever is oscillated below its resonant frequency, and, for each individual tap, a small indentation $(2 \mathrm{~nm})$ is made on the surface. Here, the peak force is used as the feedback signal and recorded for each load-unload curve. This makes it possible to generate material property maps simultaneously and with the same resolution as the height image. The AFM tip radius was measured using a standard rough sample (Ti) and Nanoscope Analysis software (tip radius was equal to $25 \mathrm{~nm}$ ). The Poisson's ratio value of 0.3 was used for all composite films. Images consisted of $512 \times 512$ pixels. Scanning rate was $1 \mathrm{~Hz}$ at all times and the peak force was set such that the resultant average deformation in each scan line was not more than $3 \mathrm{~nm}$. These settings were kept constant for all AFM images. Other scanning parameters such as integral and proportional gains were automatically set by the Nanoscope software. All the offline analyses on AFM images were conducted using Nanoscope Analysis software on height images.

The raw AFM force curve is a plot of the position of the piezo $(z$ in $n m$ ) that runs the AFM versus the output voltage of the photo-detector $(\Delta$, in $V)$. For this to be converted to a more common force-indentation height $(F-h)$ curve, the deflection sensitivity and spring constant of the cantilever must be known before nanoindentation. The spring constant of the cantilever used here was the one measured by the manufacturer $(k=311 \mathrm{~N} / \mathrm{m})$. The use of a high spring constant cantilever prevents lateral deformation and makes it possible to record all force data only in the vertical direction. The deflection sensitivity (the distance that the cantilever is really deflected for a measured change in the photodetector voltage, in $\mathrm{nm} / \mathrm{V}$ ), which is used to convert the units of $\Delta$ to $\mathrm{nm}$, was measured by indenting a sapphire substrate $(163.96 \mathrm{~nm} / \mathrm{V})$. This was repeated at least at five positions to make sure the value was consistent. Force $(F)$ was then calculated in $n N$, by multiplying the deflection of the cantilever by its spring constant, $k(F=k \Delta)$. Indentation height ( $h$, value of deformation) was calculated by subtracting the cantilever deflection from the vertical piezo position $(z)$. It is worth noting that determination of zero contact point is an important and controversial subject. Here we considered the initial contact as the point where interactions became attractive, and no sign of adhesion-induced indentation was observed (Song et al. 2008).

For each sample total of 81 indentation points with $1 \mu \mathrm{m}$ vertical and horizontal distance (large enough to avoid the effect of neighboring plastic deformation regions) were made on a $10 \times 10 \mu \mathrm{m}^{2}$ area and the average modulus was reported. The typical roughness of the surface was less than $3 \mathrm{~nm}$ for a $10 \times 10 \mu \mathrm{m}^{2}$. The sample thickness was more than 10 times larger than the indentation depth to make sure the substrate was not affecting the results. Nanoindetation frequency (which is related to the loading rate) and maximum applied load were varied between 0.1-1 $\mathrm{Hz}$ and 5-30 $\mu \mathrm{N}$, respectively in order to identify the optimum experimental parameters for samples and contact mechanics models applied.

\subsection{Calculation of Nanomechanical Properties}

Various contact mechanics models have been suggested for calculation of the elastic modulus from AFM nanoindentation data. These include Hertz (Hertz 1981), JohnsonKendall-Roberts (Johnson et al. 1971), Derjaguin-Muller-Toporov (Derjaguin et al. 1975) 
and Dimitridas (Dimitridas et al. 2002). Hertz model assumes perfectly elastic deformation in the indentation area, small contact radius in comparison to tip radius, non or small adhesion between the tip and the sample (in comparison to the applied load), spherical tip end and planar film with infinite thickness. Based on the Hertz model, contact radius $(a)$ and sample deformation or indentation depth $(h)$ are:

$$
\begin{aligned}
& a=\sqrt[3]{\frac{R F}{E_{\text {red }}}} \\
& h=\frac{a^{2}}{R}
\end{aligned}
$$

where, $R, F$ and $E_{\text {red }}$ are tip radius, applied force and reduced elastic modulus, respectively. Combining Equations 4.1 and 4.2 give Equation 4.3, which relates the indentation depth to the applied force:

$$
F=E_{\text {red }} \sqrt[3]{h} \sqrt{R}
$$

where $v_{s}$ and $v_{t i p}$ are the Poisson's ratio of the sample and the tip respectively:

$$
E_{\text {red }}=\left(\frac{1-v_{s}^{2}}{E}+\frac{1-v_{t i p}^{2}}{E_{t i p}}\right)^{-1}
$$

By assuming infinite elastic modulus for the tip $\left(E_{t i p}\right), \mathrm{E}_{\text {red }}$ is equal to the Young's modulus of the sample $(E)$ with a good approximation. Although in most cases Hertz model is applied to the loading part of indentation data to extract the elastic properties of the sample (Chizhik et al. 1998; Jee and Lee 2010), some reports exist in the literature, where the average curve from approach and retract was considered (Domke and Radmacher 1998). In this work, the Hertz model (Equation 4.3) was applied to both loading and unloading curves and the resultant elastic moduli were compared to the elastic moduli obtained from tensile tests. It is worth mentioning that application of the Hertz model is valid only if there is no adhesion between the tip and the samples. To satisfy this condition, high spring constant cantilever was selected, which could overcome the adhesion between the AFM and the sample surface more easily. Another important assumption in the Hertz model is that the contact radius $(a)$ should be small in comparison to the tip radius $(R)$. It has been previously shown that this assumption can be ignored in AFM nanoindentation experiments with indentation depths up to $200 \mathrm{~nm}$ (Chizhik et al. 1998; Tsukruk et al. 1998).

One should consider that the model used here assumes a circular shape nanoindentation on the sample surface. It has been shown by several reports that such model leads to satisfactory predictions of the elastic modulus in experiments where the indentations are not shallow (few tens on nanometers) (Domke and Radmacher 1998; Tsukruk et al. 1998; Bischel et al. 2000; Du et al. 2001; Beak and Leggett 2002; Cappella et al. 2005; Tranchida and Piccarolo 2005). In order to accurately model the indentation geometry (conical in this case), the use of Sneddon's modified Hertz model (Sneddon 1965) has been suggested. 


\subsection{Results and Discussions}

\subsubsection{Effect of Loading Rate}

One of the main assumptions of the Hertz model is that purely elastic deformation occurs at the contact region. For this reason, before applying this model to any nanoindentation data, one should verify that the contribution of plastic and viscoelastic deformations are negligible and that indentation is dominated by elastic contact. To find the optimal loading rate, elastic recovery parameter $(\xi)$ was calculated (Tranchida et al. 2006) at maximum load of $30 \mu N$ and different loading rates or in other words different AFM indentation frequencies $(0.1$ to $1 \mathrm{~Hz})$ :

$$
\xi=1-\frac{i}{h_{\max }}
$$

Here, $i$ is the residual indentation depth imaged immediately after nanoindentation and $h_{\max }$ is the penetration depth under maximum load. In the case of fully plastic deformation, $\xi=0$; and in the case of fully elastic deformation, where no imprint is left behind after the load is removed, $\xi$ will be equal to 1 . Figure 4.1 , shows the variation in the elastic recovery parameter and change in elastic moduli (calculated from the loading curve) of pure PVA film with respect to the loading rate. At loading rate $<0.4 \mathrm{~Hz}, \xi$ is less than 0.85 and there is no consistency in mechanical property measurements. This can be explained by the fact that at low loading rates, there is more time for material relaxation to occur. However, at higher loading rates, the material has less time to respond to the load through chain sliding. Therefore, loading rates above $0.4 \mathrm{~Hz}$ result in $\xi$ values above 0.9 and consistency in the elastic modulus measurements. Thus, a loading rate equal to $1 \mathrm{~Hz}$ was selected for further indentation tests to maximize $\xi$. Higher loading rate than $1 \mathrm{~Hz}$ was not selected to avoid the over-shot effect (Yang and Zhang 2004). Also, it is worth noting that due to the viscoelastic properties of polymers, low loading rates can result in a phenomenon called nosing, where the slope of initial parts of the unloading curve will be negative (Oyne and Cook 2003; Zhang et al. 2005). Nosing can be avoided by conducting the indentation tests at relatively fast loading-unloading rates or by repeating indentation at the same spot for a few times (Klaperich et al. 2001). No nosing was observed in the current study. 


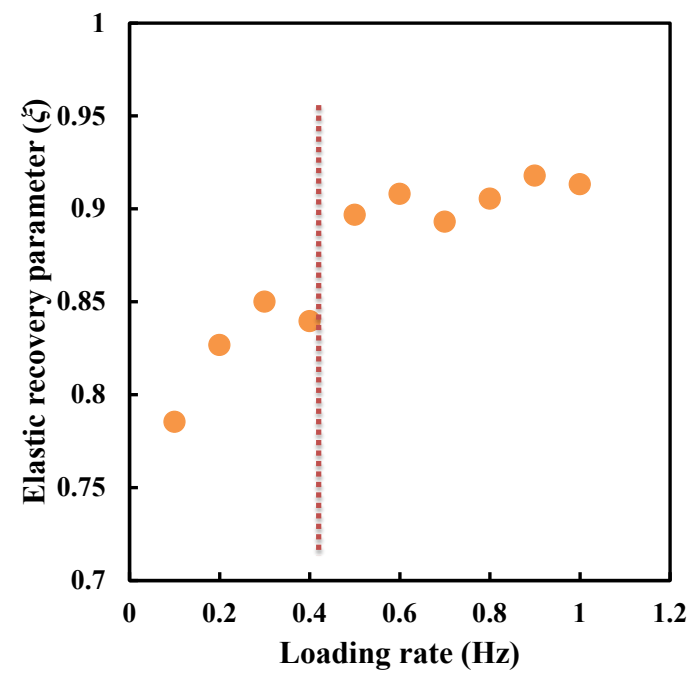

(a)

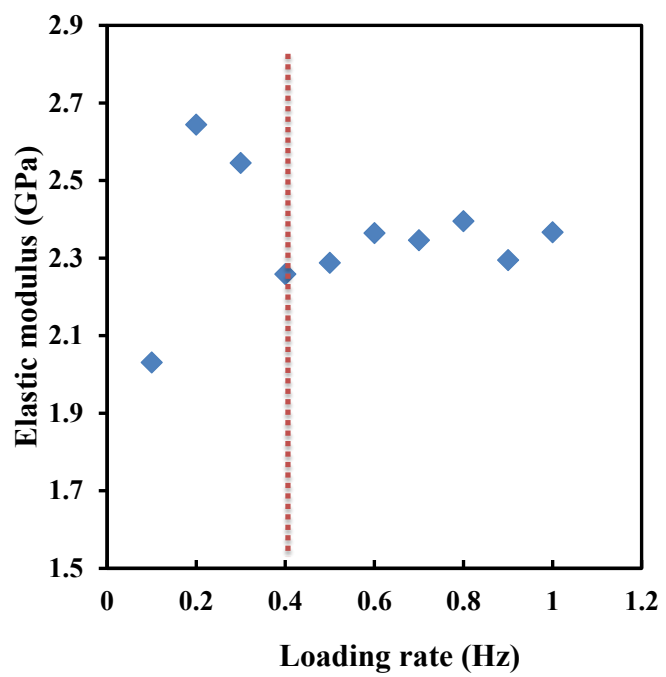

(b)

Figure 4.1 (a) Effect of loading rate on the elastic recovery parameter ( $\xi$ ). (b) Variation of modulus as a function of loading rate. The error bars show the standard deviations. Loading rate $>0.4 \mathrm{~Hz}$ results in higher $\xi$ and consistency in elastic property measurements. The red dashed line shows the optimal value after which loading rate is high enough to eliminate most of plastic and viscoelastic deformations.

\subsubsection{Effect of Maximum Indentation Load}

In nanoindentation studies, an important parameter that can determine the accuracy of measurements and scattering in the data is the maximum applied load. This load should be low enough so that the penetration on the surface is not larger than one-tenth of the thickness of the sample (to avoid the effect of the underlying substrate) (Miyake et al. 2006). On the other hand, the applied load should be large enough to decrease experimental uncertainties and measure the real elastic properties of the sample without the effect of surface roughness (Klapperich et al. 2001). Figure 4.2a and b demonstrate the variation of modulus (from the loading curve) at the surface of PVA film and the corresponding indentation imprints as the maximum indentation load was increased, respectively. The nanoindentation elastic modulus values reported in Figure 4.2a are average values of at least five indents and error bars at specific data points represent the standard deviation of the mean as measured through repetitive tests. It appeared that at indentation loads lower than $11 \mu \mathrm{N}$, the elastic modulus depended on the applied load, while indentation forces greater than $11 \mu N$ resulted in consistent elastic modulus values. This can be explained by the fact that by increasing the applied load, the penetration depth increases and as a result the effect of microscopic roughness and various microstructures vanishes. Therefore, for the subsequent nanoindentation tests the maximum load of $30 \mu N$ was selected. 

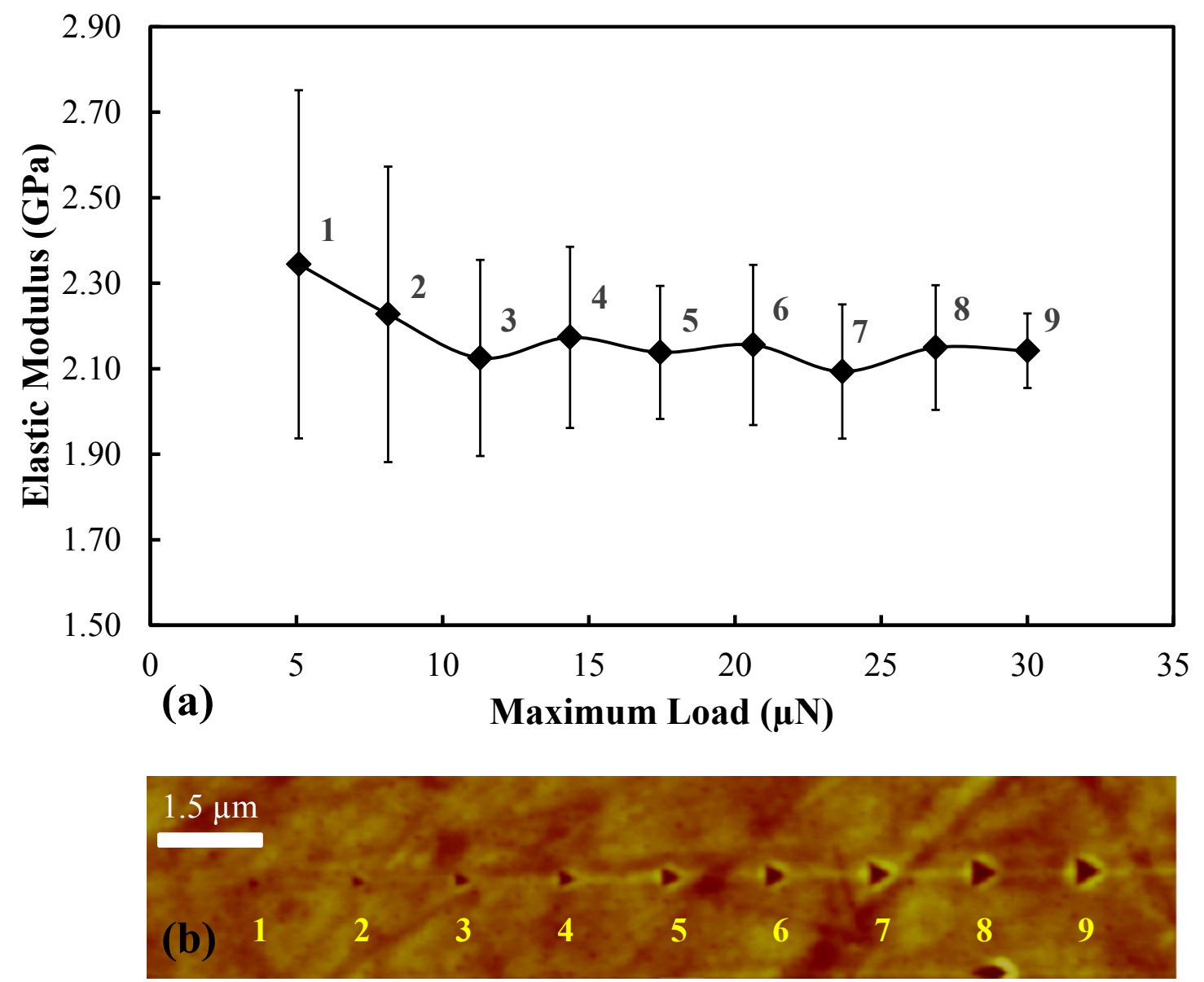

Figure 4.2 (a) Effect of maximum applied load on the resultant modulus values. (b) Corresponding indentation imprints shown by numbers in (a). At indentation loads lower than $11 \mu N$, elastic modulus varied with the applied load. On the other hand, when applied loads are greater than $11 \mu N$, consistent results were obtained.

\subsection{Nanomechanical Properties of PVA-PAA-CNC Membranes}

Figure 4.3 shows the AFM images of PVA membranes reinforced with CNCs before (Figure 4.3, a-e) and after nanoindentation experiments (Figure 4.3, f-j). In general, the average roughness values of the samples were less than $3 \mathrm{~nm}$. CNCs were easily detected on the topography images (shown by yellow arrows in Figure 4.3, c-e) and were mostly found in groups and aggregates rather than single whiskers. Such CNC agglomerations have been reported in several cases before (Choi and Simonsen 2006; Bodenson and Oksman 2007), and are believed to be due to CNCs' high tendency to form hydrogen bonds.

AFM images after nanoindentation showed no significant sign of pile-up or sink-in in the pure PVA and PVA 90-PAA 10-CNC 0 films, while pile-up (where free surface of material bulged out during indentation) was found at the indentation regions in composites that contained $\mathrm{CNC}$, and it increased with $\mathrm{CNC}$ content. This can be seen 
clearly in three dimensional AFM topography images shown in Figure 4.4. Increase in pile-up with increase in reinforcement content can be due to the restriction of movement imposed on polymer chains by high elastic modulus CNC crystals and has been observed in other nanocomposite systems (Nagy et al. 2006).
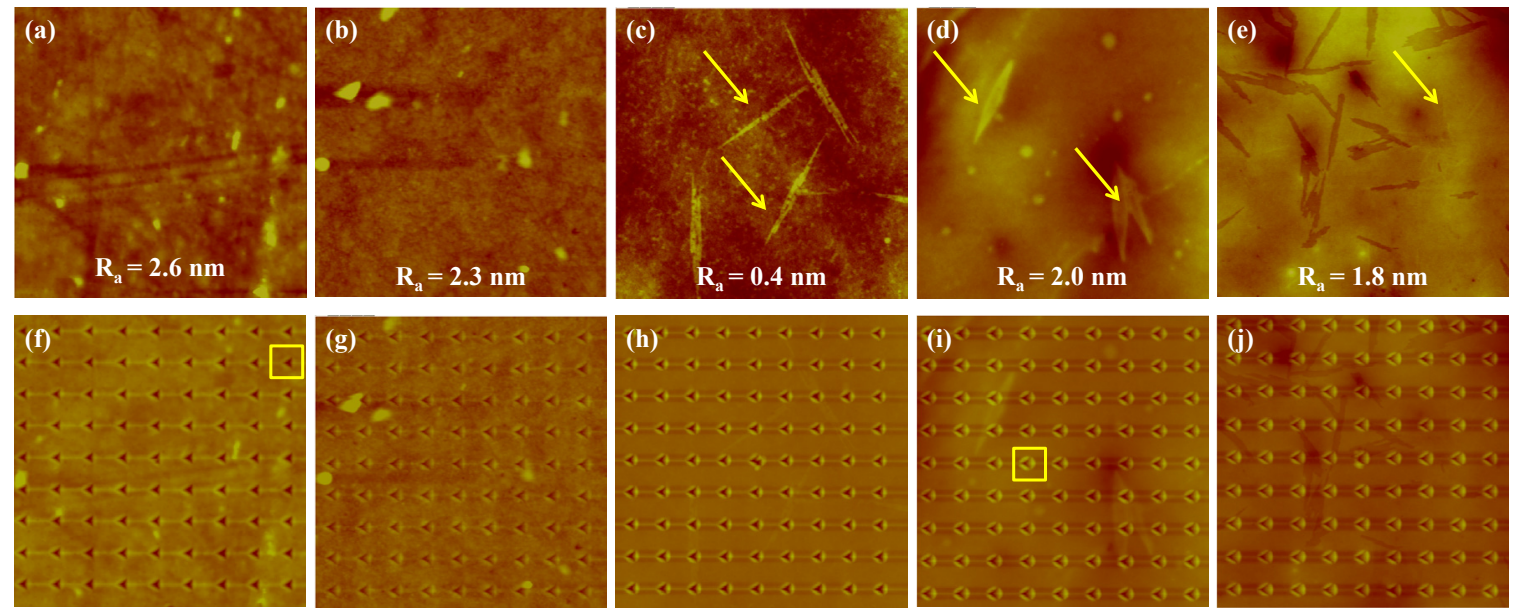

Figure 4.3 AFM topography images of $10 \times 10 \mu m^{2}$ area: (Top) before and (bottom) after nanoindentation. (a ,f) PVA 100-PAA 0-CNC 0; (b, g) PVA 90-PAA10-CNC 0; (c, h) PVA 85-PAA 0-CNC 15; (d, i) PVA 80-PAA 10-CNC 10; and (e, j) PVA 70-PAA 10CNC 20. CNCs are pointed with yellow arrows in (c), (d) and (e). Average roughness values $(\mathrm{Ra})$ are shown on height images before nanoindentation. Areas in yellow squares in (f) and (i) are shown in 3D in Figure 4.4.

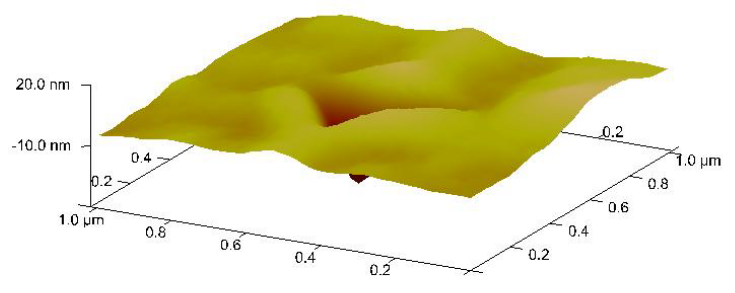

(a) PVOH 100- PAA 0-CNC 0

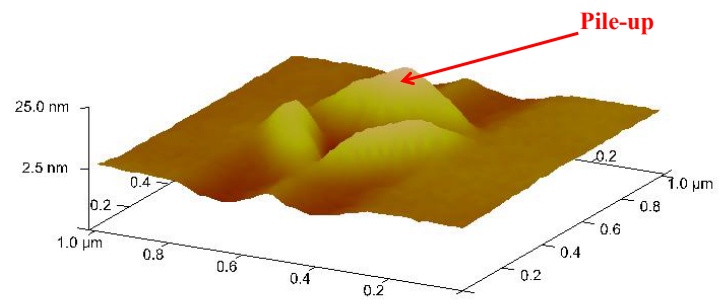

(b) PVOH 80- PAA 10- CNC 10

Figure 4.4 High magnification three dimensional AFM topography images of nanoindentation imprints at $1 \times 1 \mu \mathrm{m}^{2}$ areas shown with yellow squares in Figure 4.3. (a) PVA 100-PAA 0-CNC 0 with no significant pile-up and (b) PVA 80-PAA 10-CNC 10 with pile-up around the indentation imprint.

In nanoindentation studies using Oliver-Pharr method (Oliver and Pharr 1992), it has been shown that if the residual/maximum indentation ration $\left(i / h_{\max }\right)$ is higher than 0.7 , pile-up can cause an increase in the contact area and as a result overestimation of elastic modulus and hardness calculations up to 60\% (Bolshakov and Pharr 1998). On the other hand, Tranchida et al. (2007) have shown, for various polymer films (acstic polystyrene, polycarbonate and isotactic polypropylene) with different mechanical properties, that pile-up is not expected to cause failure in measuring the mechanical properties at low 
penetration depths. Although Oliver-Pharr method was not used here, $i / h_{\max }$ was kept bellow 0.7 at all times (Section 5.1), thus no significant effect of pile-up on the measurements was expected (Bolshakov and Pharr 1998; Tranchida et al. 2007).

Representative Force-Indentation depth $(F-h)$ curves in the five nanocomposite membranes that were discussed in Figure 4.3 are shown in Figure 4.5. With the exception of PVA 85-PAA 0 -CNC 15 film, the $F$ - $h$ curves appeared to be similar on all the samples and only two differences were observed. One is related to the slope of $F$ - $h$ curves, which indicates the differences in mechanical properties. Second, the maximum indentation depth was different, meaning that under the same maximum load, softer samples deform more than harder ones. In all the curves, pop-in and kick-back events were observed. Although the origin of these events is not clear, similar observations have been made for metals and they have been related to different mechanisms such as microfracture, sudden nucleation of dislocations, or sudden release of energy during loading and unloading (Asif et al. 2000 ; Miller et al. 2004; Gaillard et al. 2006).

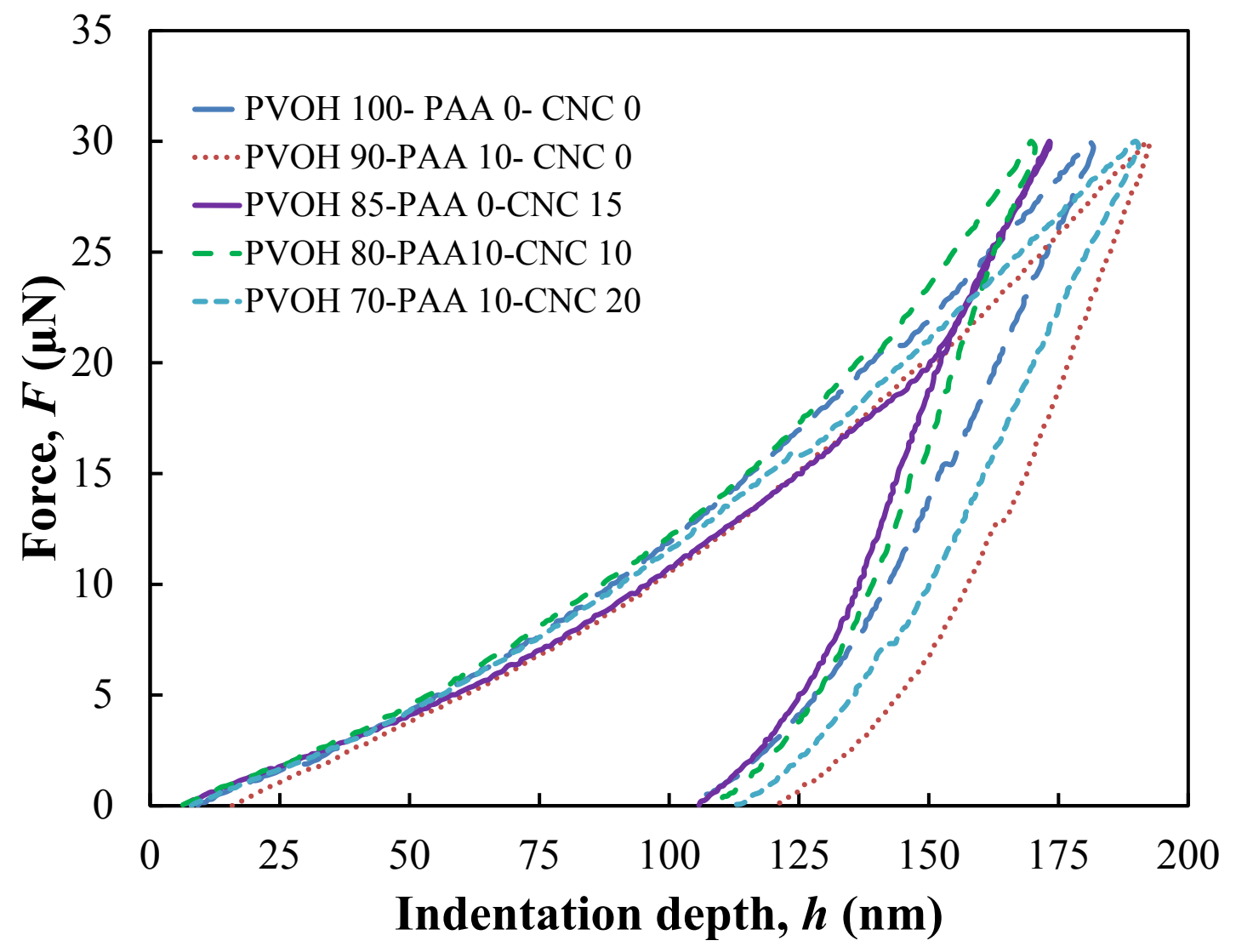

Figure 4.5 Representative indentation curves on pure PVA and four nanocomposite films with different $\mathrm{CNC}$ and PAA content. The difference in the slope of the curves shows the difference in the elastic moduli. Pop-in and kick-back points on the curves occur as a result of sudden energy releases during loading and unloading, respectively. 
Two representative $F$ - $h$ curves obtained from nanoindentations on PVA 80-PAA 10CNC 10 at two different locations, one at polymer matrix (black curve) and another on CNC (blue curve), are shown in Figure 4.6. There are a few small differences between these two curves. First, at the same maximum load, the slope of the $F-h$ curve is higher and less deformation is observed when the CNC is indented. Second, the unloading part of the indentation curve on polymer matrix indicates that an adhesion force of $1 \mu \mathrm{N}$ (shown by red arrow) has been detected by the AFM cantilever. These two observations can be related to the rigid crystalline structure of $\mathrm{CNC}$ and its high elastic modulus (Tanaka and Iwata 2006), as compared to the soft polymer matrix. It is also worth noting that, the difference between these two curves is not substantial and this comes from the fact that $\mathrm{CNC}$ is embedded in the polymer matrix and that the applied load is not very high. Because of high mechanical properties of $\mathrm{CNC}$, one expects that the polymer underneath the $\mathrm{CNC}$ contributes to the recorded deformation behavior. While only these two curves are presented here, the same trend was observed on different areas on the sample and on different membrane compositions.

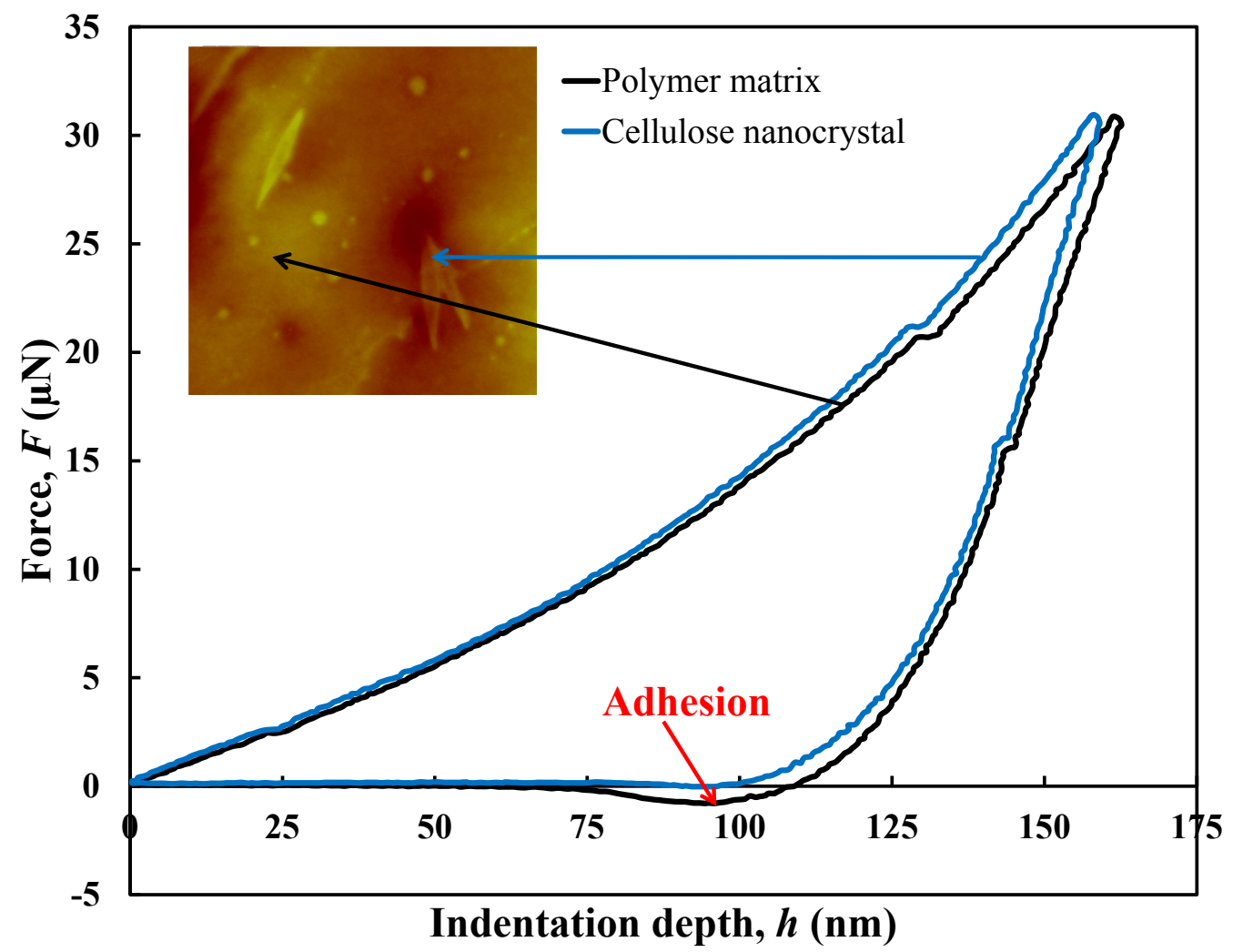

Figure 4.6 Representative indentation curves on PVA 80-PAA 10-CNC 10 at two different locations. The blue curve represents the indentation on a $\mathrm{CNC}$, while the indentation of the polymer matrix is shown by the black curve. The indentation locations are shown on the topography image by the arrows. There is a small difference between these two curves in terms of maximum penetration under the applied load, slope and adhesion, but these differences are not substantial. 


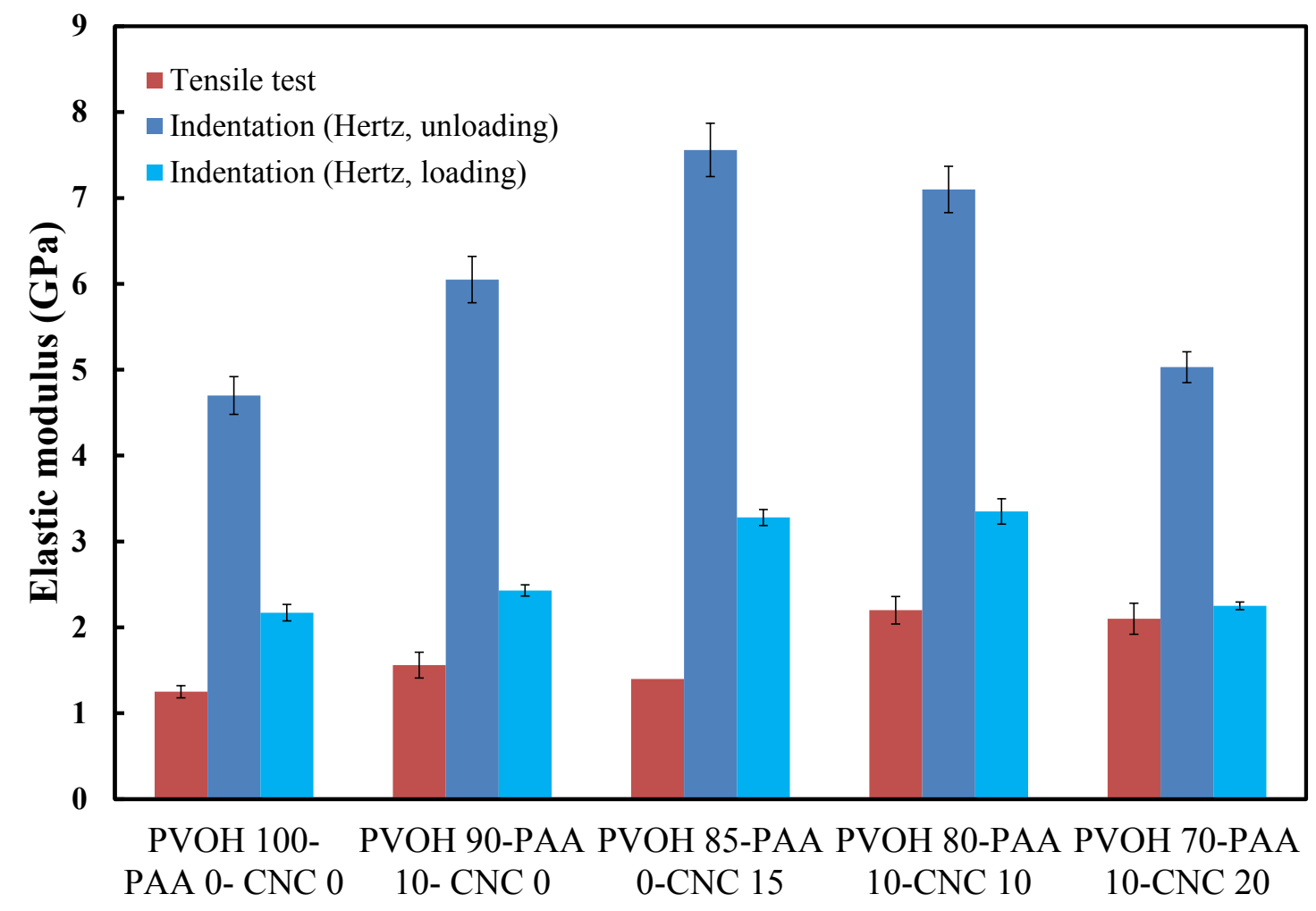

Figure 4.7 Comparison of the elastic modulus of nanocomposite films (PVA 100-PAA 0CNC 0, PVA 90-PAA 10-CNC 0, PVA 85-PAA 0-CNC 15, PVA 80-PAA 10-CNC 10, PVA 70-PAA 10-CNC 20) obtained from tensile test (red) and nanoindentation by fitting the Hertz model to loading (light blue) and unloading (dark blue) curves. Nanoindentation results from fitting the Hertz model on the loading curve resulted in values closer to bulk mechanical properties.

Next, the elastic moduli obtained from tensile test and nanoindentation tests were compared (Figure 4.7). The nanoindentation elastic modulus values reported in Figure 4.7 are average values of at least 81 indentation points and error bars at specific data points represent the standard deviation of the mean as measured through repetitive tests. The elastic moduli obtained from fitting the Hertz model to unloading and loading nanoindentation curves were greater than those obtained from tensile tests, with a factor of 1.07 to 2.34 for the latter (Figure 4.8). Such behavior has been reported for other polymer systems (VanLandingham et al. 2001; Zimmerman et al. 2005) and there could be multiple reasons behind this. On simple reason is that the available free volume (which is related to the elastic modulus of polymers) is less in nanoindentation tests. Tensile tests are performed on a much larger volume than nanoindentation, thus there are more defects such as microscopic cracks, which result in lower modulus values. Also, compression modulus is measured through nanoindentation and it is possible that it is larger than macroscopic values measured through tensile test. And finally, and most probably loading rates in tensile and nanoindentation tests are different and it is well 
known that the elastic modulus can be highly affected by the strain rate in many materials. It should be noted that 81 indentation tests were performed on each sample, which consisted of both polymer matrix and CNCs. The results reported in the manuscript are the average values, thus including the properties of the composite material. Additionally, although the AFM tip radius $(25 \mathrm{~nm})$ was smaller than the size of the crystals, the indentation depths were much larger $(200 \mathrm{~nm})$. At this indentation depth, the part of the AFM tip in contact with the sample has much larger radius than the AFM tip.

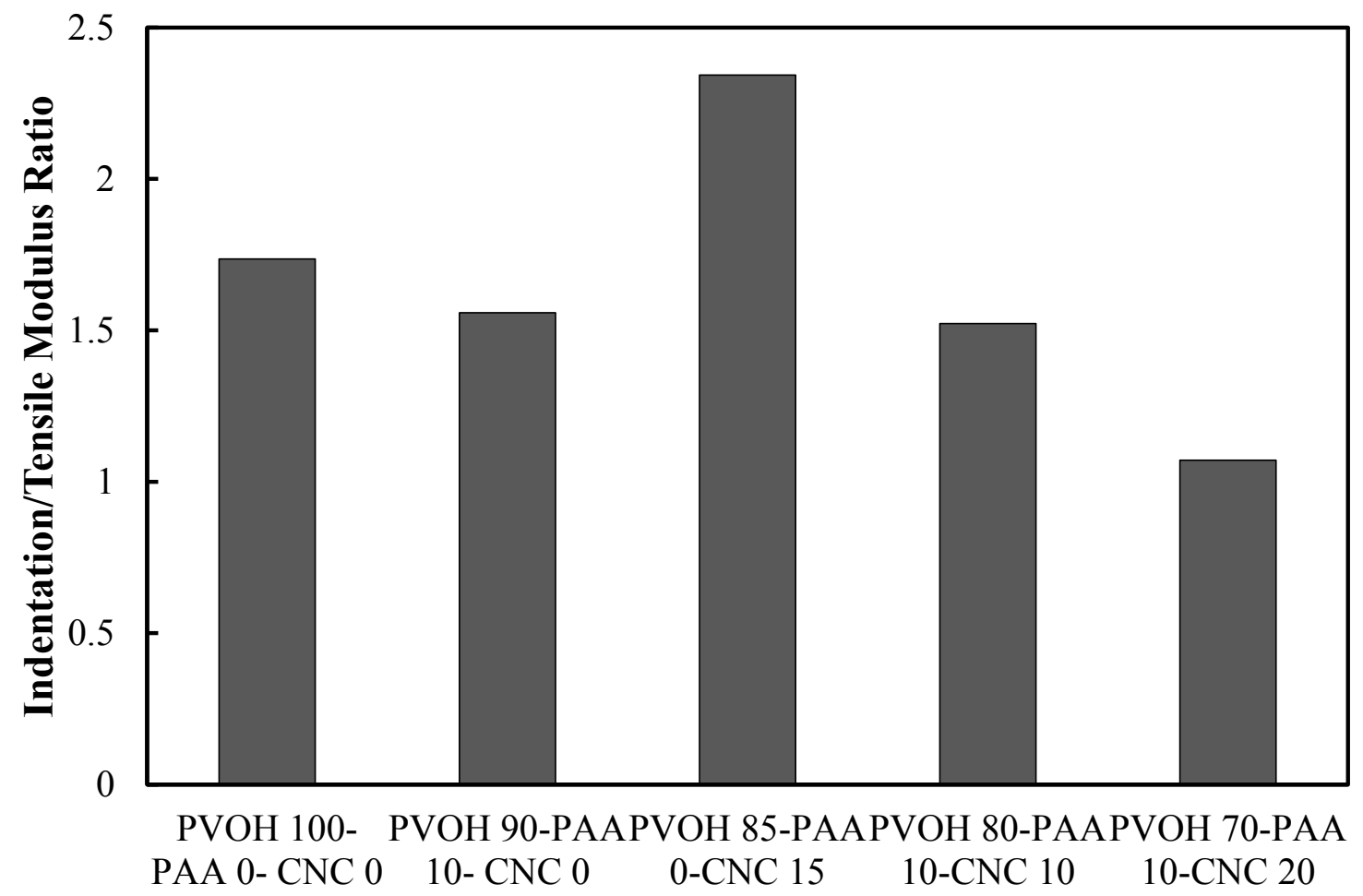

Figure 4.8 Indentation/tensile elastic modulus ratio in different PVA nanocomposites. Nanoindentation/tensile modulus ratio was larger than 1 for all samples. In the nanoindentation method, the elastic modulus was calculated by fitting the Hertz model to the loading curves.

Overall, the nanoindentation results obtained by fitting the Hertz model to the loading curves resulted in values closer to macro-scale tensile properties than the unloading curves. One explanation can be the assumptions made in the Hertz model, which consider fully elastic contact deformation and zero adhesion between the tip and the sample. These assumptions are applicable to the loading curve and less so to the unloading section, which may be partially plastic and may contain some adhesion forces (see Figure 4.6). Another reason can be unavoidable viscoelastic creep in the samples. Although creep was negligible in this study (high loading rate reduced the ability of the polymers to deform in a plastic or viscoelastic manner), it is a common property of polymers and may have increased the initial slope of unloading curves and as a result the calculated elastic moduli (VanLandingham et al. 2001). One should note that at the initial portion of the unloading 
curves, these curves are linear. As such, fitting the linear $F-h$ curve with a nonlinear Hertzian contact model of $F=E_{\text {red }} \sqrt[3]{h} \sqrt{R}$ may be the reason for some of the discrepancies between the elastic modulus extracted form the fitting of the unloading curves, when compared to the tensile tests. Therefore, according to the ASTM E 2546-07 (Standard practice for instrumented indentation testing), the upper $80 \%$ of the unloading curves should be used for fitting (linearly or power law) (Fischer-Cripps 2004).

In all the samples except for the PVA 85-PAA 0-CNC 15, nanoscale elastic moduli follow the same trend as the ones obtained from the macro-scale tensile tests. Macroscale tensile data indicates that the addition of CNC and PVA up to $10 \mathrm{wt} \%$ resulted in the highest elastic modulus $(2.2 \mathrm{GPa})$ in PVA membranes. When $\mathrm{CNC}$ is added to a polymer, above the percolating threshold, a firm three dimensional network is formed by the crystals (because of the intermolecular hydrogen bonds that form between them). This three dimensional network keeps the polymer chains in the matrix from deforming and increases the macro-scale elastic modulus (Favier et al. 1997). Tensile tests showed that PVA 85-PAA 0-CNC 15 had the lowest modulus value (1.4 GPa) in comparison to other composite films and previous studies have shown that the agglomeration of excess CNCs is responsible for the observed degradation in macro-scale properties (Choi and Simonsen 2006; Bodenson and Oksman 2007; Paralikar et al. 2008). The macro-scale results indicated that $\mathrm{CNCs}$ were most effective in improving the mechanical properties when PAA was present. With no PAA, CNCs with concentrations that exceed $10 \mathrm{wt} \%$ degraded the mechanical properties (PVA 85-PAA 0-CNC 15). On the other hand, when PAA was added to the PVA, and CNC content was increased to $20 w t . \%$ (PVA 70-PAA 10-CNC 20), macro-scale mechanical properties were slightly lower than the PVA 80PAA 10-CNC 10 case.

The only discrepancy between the nanoindentation results and the macro-scale tensile tests were observed for the PVA 85-PAA 0-CNC 15 sample. The macro-scale test showed that this sample had the lowest elastic modulus while the nanoindentation results gave completely opposite indication. This seems to be puzzling at the first sight but can be explained by the high degree of local heterogeneity of CNCs' distribution in the sample. Due to lack of PAA, the CNCs' dispersions in the PVA are quite heterogeneous. In fact, Figure 4.9 shows the AFM images obtained by scanning two different locations and confirms such high degree of inhomogeneity in this sample. Therefore, a local probing technique such as AFM will not be able to capture the true macro-scale properties in highly heterogeneous samples while macro-scale tests are less sensitive to such nanoscale spatial variations because of larger sampling scale. 


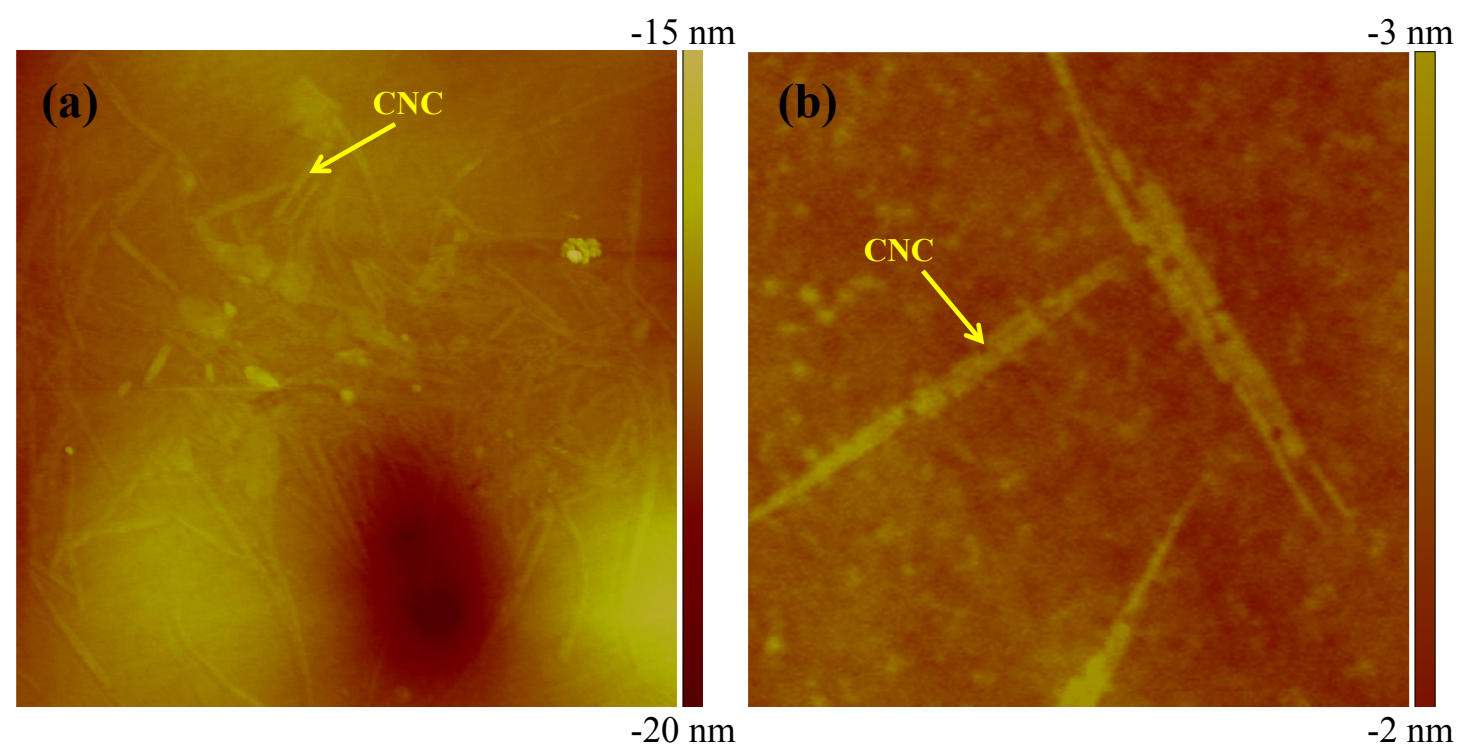

Figure 4.9 Topography images from two different location $(a, b)$ on the PVA 85-PAA 0CNC 15 sample, show high degree of inhomogeneity in the sample. Scan area is $5 \times 5 \mu \mathrm{m}^{2}$. Yellow arrows show CNCs embedded in PVOH.

\subsection{Conclusions}

The aim of this paper was to determine the nanomechanical properties of PVA-PAA$\mathrm{CNC}$ nanocomposite membranes and to compare them to those at the macro scale. For this purpose, tensile tests and nanoindentation using an AFM system were performed on composites with CNC and PAA contents of 0 to $20 \mathrm{wt}$. $\%$ and 0 to $10 \mathrm{wt}$. $\%$, respectively. It was shown that in order to satisfy the Hertz elastic model, a maximum load of $30 \mu \mathrm{N}$ should be used with $1 \mathrm{~Hz}$ loading rate.

Comparison of the mechanical properties verified that elastic moduli from nanoindentation followed the same trend as those obtained from tensile tests for all samples except PVA 85-PAA 0-CNC 15. Tensile tests showed that addition of CNC up to 20 wt. \% improved the elastic modulus of PVA, when PAA was present in the composition. Also, in the absence of PAA, agglomeration of CNCs resulted in nanocomposite membrane with lowest elastic modulus. On the other hand, nanoindentation tests showed that addition of only $15 \mathrm{wt} \% \mathrm{CNC}$ improved the elastic modulus of the composite similar to the case where $10 \mathrm{wt} \% \mathrm{CNC}$ and $10 \mathrm{wt} . \% \mathrm{PAA}$ was added to PVA. This discrepancy between macro and nano-scale mechanical properties comes from the high degree of local heterogeneity of $\mathrm{CNC}$ distribution in PVA 85-PAA 0-CNC 15 sample.

Because of the differences in loading conditions and the volume of the sample under experiment, in comparison to tensile tests, nanoindentation resulted in larger elastic moduli values. In the Hertz modeling framework, the incorporation of the loading curves resulted in closer values to tensile elastic moduli compared to the unloading data. It was found that the composites reinforced with cellulose nanocrystals had smaller indentation 
imprints and the pile-up effect increased with the increase of cellulose nanocrystal content.

\subsection{Acknowledgements}

The authors acknowledge the National Science Foundation (NSF) for the grants No. 0820884 and No. 11000806/1100572 from the Division of Materials Research (DMR). 


\section{Gradient of Nanomechanical Properties in the Interphase of Cellulose Nanocrystal Composites ${ }^{4}$}

\subsection{Introduction}

Poly(vinyl alcohol) (PVA) is a water soluble polymer. It is largely produced and has been extensively used as a membrane since it is a good barrier against oxygen and aromas and is resistant to permeation of solvents and oils. PVA barrier membranes do not have sufficient mechanical properties (Cadek et al. 2002; Liu et al. 2005; Cheng et al. 2007) and have poor stability at high moisture contents (Roohani et al. 2008). This happens because water molecules swell PVA and degrade its barrier functionality. Paralikar et al. (2008) have shown that the addition of $10 \mathrm{wt} \%$ cellulose nanocrystals (CNCs) to PVA and cross linking it using $10 w t$. \% poly (acrylic acid) (PAA) improves the overall mechanical properties of the composite. They showed that PAA acted as a cross-link agent and an ester linkage was formed between the carboxyl groups in PAA and hydroxyl groups in CNC and PVA, which resulted in a highly networked structure with improved properties.

One of the drawbacks of using CNCs is their high tendency to agglomerate due to the large number of hydroxyl groups on their surface (highly polar and hydrophilic). This makes dispersion of these crystals very difficult in polymer matrices, especially those that are non-polar or hydrophobic. In this case the properties of interfacial zone or interphase can play a major role in overall properties of the cellulose nanocrystal composite materials. This interphase represents a heterogeneous transition zone, which separates the bulk polymer from the CNC reinforcement. In general the size of interphase can vary between nanometers to micrometers (Drzal 1986). Interphase is formed due to local physical or chemical changes (cross linking, immobilization, crystallization of polymer, interdiffusion of atoms or molecules, etc.) that are dictated by the surface of the reinforcement. In the literature one can find several approaches and characterization techniques to identify the interphase properties. These include nuclear magnetic resonance spectroscopy (NMR) (Droste et al. 1971), Fourier transform infrared spectroscopy (FTIR) (Ishida and Koenig 1978), ion scattering spectroscopy (ISS) and secondary ion mass spectroscopy (SIMS) (Dibenedetto and Scola 1978), X-ray photoelectron spectroscopy (XPS) (Thomson and Dwight 1999), stereoscopic displacement analysis (Williams et al. 1990), and nanoindentation (Hodzic et al. 2000; Williams et al. 1990).

One of the limitations of the above techniques is their spatial resolution. Therefore scanning probe microscopy (SPM) techniques, and particularly atomic force microscopy

4 The material contained in this chapter has been accepted for publication in Composites Science and Technology. See Appendix C for proper documentations. 
(AFM) with resolution less than $0.1 \dot{A}$, can fill this gap. First attempts for characterization of the interphase using AFM were based on the contact and force modulation modes (Mai et al. 1998). Force modulation involves modulating the tip-sample interaction force as the AFM tip moves across the sample surface in contact mode. AFM has also been used as a nanoindenter (VanLandingham et al. 1997; Winter and Houston 1998) to characterize the mechanics of the interphase region. For this purpose either an indenter probe is attached to the end of a capacitive load sensor or a stainless steel cantilever with a diamond tip is used. Another approach for characterization of the interphase in AFM is based on phase imaging, which is similar to force modulation techniques but is performed in tapping mode (Downing et al. 2000). The phase image is generated simultaneously with the topographic image as a result of changes in the phase angle of the cantilever due to change in topography, tip-sample interaction, and experimental conditions (Raghavan et al. 2000). The sine phase image contains only the qualitative data; it does not provide a basis to accurately measure the properties of the interphase region.

To the authors' knowledge, no reports have documented the quantitative mechanical properties of the interphase in cellulose-composite systems. Here, the purpose was to study the mechanical properties of the interphase region in CNC-based nanocomposites. Peak force tapping mode (PF-TM) was used for obtaining quantitative nanomechanical (QNM) maps of the interphase in PVA-PAA-CNC composite films. This work demonstrates, for the first time, the direct measurements of the gradient in adhesion and elastic modulus in CNC-based nanocomposites at the nanoscale interphase. Lastly, the effect of CNC size was investigated on the interphase thickness.

\subsection{Materials and Methods}

Materials studied in this work were prepared as described in Paralikar et al. (2008). Briefly, CNCs were prepared by partial acid hydrolysis of cotton (Whatman \#1 filter paper, Clifton, NJ). Filter paper was ground and stirred with $65 \% \mathrm{H}_{2} \mathrm{SO}_{4}(v / v)$ for $50 \mathrm{~min}$, at $45{ }^{\circ} \mathrm{C}$. The cotton-to-acid ratio was 1:10 g.ml $\mathrm{l}^{-1}$. After the mixture was centrifuged five times, it was subjected to ultrasonication (Branson Sonifier, Danbury, CT) for $15 \mathrm{~min}$, in order to break agglomerations and evenly disperse $\mathrm{CNCs}$ in water. To remove the remaining salts, ultra filtration was done until the conductivity was $<10 \mu \mathrm{S} . \mathrm{cm}^{-1}$, (Ultrasette tangential flow UF device, pore size $=50 \mathrm{~nm}$, Pall Corp., Ann Harbor, MI). Finally, an aqueous suspension of $1 \% \mathrm{CNC}$ was obtained by concentrating the dispersion of CNCs in a Rotavaporizer R110 (Buchi, Flawil, Switzerland). Poly (vinyl alcohol), (99+\% hydrolyzed, $\left.\mathrm{M}_{\mathrm{w}}=89000-98000 \mathrm{~g} \cdot \mathrm{mol}^{-1}\right)$ was obtained from Sigma-Aldrich Inc. (St Louis, MO). Poly (acrylic acid), $\left(\mathrm{M}_{\mathrm{w}}=2000 \mathrm{~g} \cdot \mathrm{mol}^{-1}\right)$ was obtained from Aldrich Chemical Company Inc. (Milwaukee, WI).

Solutions of PVA and PAA were prepared by dissolving their powder in DI water and stirring in an oil bath for $30 \mathrm{~min}$, at $85^{\circ} \mathrm{C}$. Calculated volume of component solutions and $\mathrm{CNC}$ dispersions ( $80 w t . \%$ PVA, $10 w t . \%$ PAA, $10 w t . \% \mathrm{CNC}$ ) were then blended and sonicated for $25 \mathrm{~min}$ to disperse any remaining aggregates. Then mixtures were casted in a flat-bottom plastic dish and air dried for $48 \mathrm{hrs}$. The samples were heat treated at 170 ${ }^{\circ} \mathrm{C}$ for $45 \mathrm{~min}$. Finally, mixtures were casted in a flat-bottomed plastic dish and air dried 
for $48 \mathrm{~h}$. A sample of $80 \mathrm{wt} . \%$ PVA and $15 \mathrm{wt} . \% \mathrm{CNC}$ was also prepared to investigate the effect of existence of PAA on the properties of the interphase.

\subsection{QNM Analysis: PF-TM in AFM}

In peak force tapping mode (PF-TM) technique, using the main $\mathrm{Z}$ piezoelement, the vertical motion of the cantilever is oscillated below its resonant frequency. For each individual tap, nanoscale material property and peak force is collected by collecting one force curve at each pixel (Figure 5.1) (Pittenger et al. 2009). Each force-separation curve is then analyzed to generate material property maps with the same resolution as the height image. PF-TM relies on the maximum interaction force between the tip and the sample (peak force) as the feedback signal. By controlling the maximum force, smaller deformation is formed on the surface $(<3 \mathrm{~nm})$, which increases the imaging resolution and decreases the tip wear.

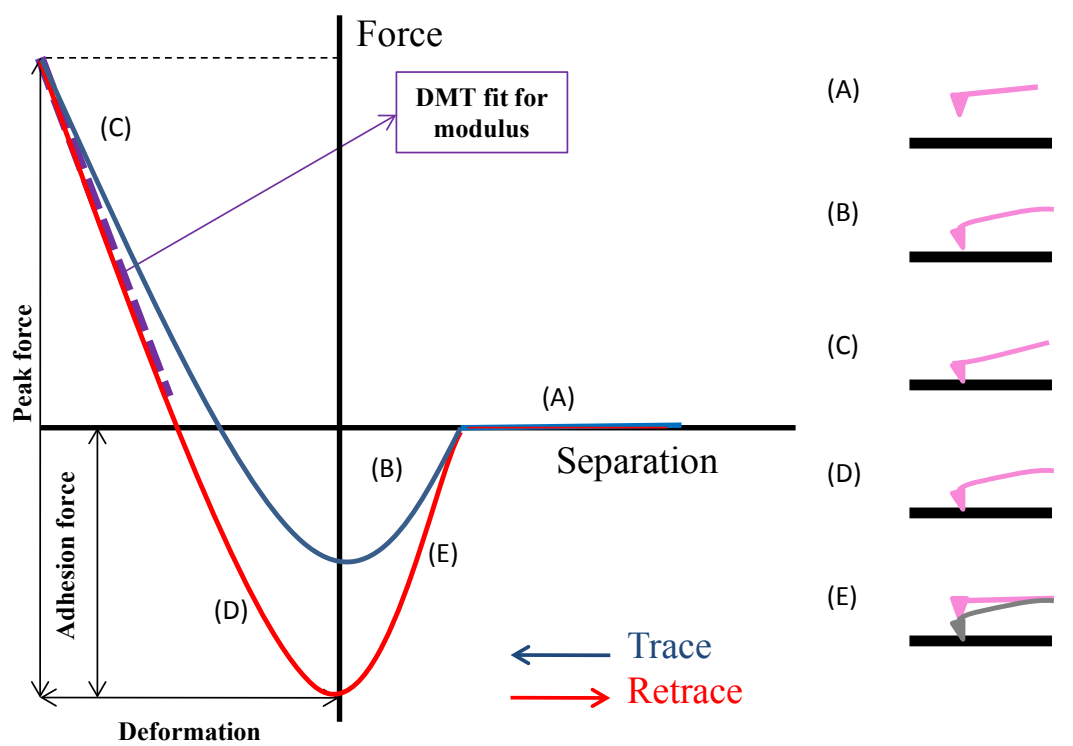

Figure 5.1 Left: Schematic of force-separation curve collected at each pixel. The blue curve (trace) represents the loading portion and the red curve (retrace) indicates the unloading part. Part of the unloading curve (bold dashed line) is used to calculate the Derjaguin-Muller-Toropov (DMT) elastic modulus (Derjaguin et al. 1975). The minimum force in the retrace curve is used for mapping the adhesion properties. Also, the peak force value is used for peak force tapping imaging in AFM. Right: tip-sample interactions during each tap. At (A) there is little or no force on the tip. As the piezo expands (separation decreases), the tip is pulled down (B) by attractive forces such as van de Waals, capillary or electrostatic, until it touches the surface (separation $=0$ ). Then the tip is pushed into the sample until the set point for the peak force is reached (C). The piezo then starts to pull up the tip and the force keeps decreasing (D) to a minimum value, where the tip comes off the surface. After this point forces affecting the tip keep decreasing as separation increases (E) and piezo returns to its initial position. 
During data acquisition the deflection of the cantilever ( $\Delta$ in $V$ ) and the position of the piezo $(z$ in $\mathrm{nm}$ ) are recorded at each tap (or pixel). In order to convert this data to force in $n N$ versus separation in $\mathrm{nm}$, the deflection sensitivity of the cantilever should be measured. This is the distance that the cantilever is really deflected for a measured change in the photo-detector voltage $\left(n m . V^{l}\right)$. Deflection sensitivity depends on the cantilever type and the laser path from the back of the cantilever to the detector. Deflection sensitivity converts the units of $\Delta$ to $\mathrm{nm}$. Force $(F)$ can then be easily calculated in $n N$, by multiplying the deflection of the cantilever by its spring constant, $k$ $(F=k \Delta)$. Separation is by definition the negative value of deformation and is calculated by adding the vertical piezo position $(z)$ to the cantilever deflection. From the force separation curve for each pixel, peak force, adhesion force, deformation, and elastic modulus values are sent to separate imaging channels. QNM imaging continues at usual imaging speed and material property maps are generated simultaneously with the height image.

On each force curve (Figure 5.1), peak force is the maximum force value and it is used as the feedback signal in PF-TM. Adhesion force is the minimum force value, which is affected by the interaction between the tip and the sample and increases with the tip end radius. Deformation is the difference of the separation from force equal to zero to the peak force. Lastly, the elastic modulus is calculated by fitting the Derjaguin-MullerToropov (DMT) model (Derjaguin et al. 1975) to the initial section of the retrace curve (bold dashed line in Figure 5.1):

$$
F=\frac{4}{3} E^{*} \sqrt{R d^{3}}+F_{a d h}
$$

where $F$ is the force on the tip, $R$ is the tip radius, $d$ is the separation, $F_{a d h}$ is the adhesion force and $E^{*}$ is the reduced elastic modulus. Knowing the Poisson's ratio of the sample $\left(v_{s}\right)$ and the tip $\left(v_{t i p}\right)$ and assuming infinite elastic modulus for the tip $\left(E_{t i p}\right)$, the elastic modulus for the sample $\left(E_{s}\right)$ can be calculated:

$$
E^{*}=\left(\frac{1-v_{s}^{2}}{E_{S}}+\frac{1-v_{t i p}^{2}}{E_{t i p}}\right)^{-1}
$$

\subsection{AFM Measurements}

Dimension(C Icon $(\mathrm{C}$ AFM was used for the force probing in this work. Silicon AFM probes with nominal spring constant of 20-80 N.m $\mathrm{m}^{-1}$ and tip radius of 5-12 $\mathrm{nm}$ were used. The deflection sensitivity of the cantilever was measured by acquiring a force curve on a hard sapphire surface. This was repeated at least at five positions to make sure the value was consistent $\left(104.75 \mathrm{~N} . \mathrm{m}^{-1}\right)$. The spring constant was acquired to be $24 \mathrm{~N} . \mathrm{m}^{-1}$, using the Sedar method (Ohler 2009). It is worth mentioning that such high spring constant cantilever was selected in order to reduce the amount of cantilever bending during AFM data acquisition.The tip radius was measured using the relative method. This briefly included scanning a standard sample (Poly Styrene) with a known elastic modulus, and changing the tip radius in Nanoscope software until the expected value $(2.7 \mathrm{GPa})$ was obtained in the elastic modulus channel. Tip radius for $2 \mathrm{~nm}$ deformation was equal to 5 
nm. Finally the Poisson's ratio value of 0.3 was used for three phases in the composite. These settings were kept constant for all the subsequent measurements.

The AFM images consisted of $512 \times 512$ pixels. Scanning rate was changed according to the scan size and was $<1 \mathrm{~Hz}$ at all times. The peak force was set such that the resultant average deformation in each scan line was not more than $3 \mathrm{~nm}$. Other scanning parameters such as integral and proportional gains were automatically set by the Nanoscope software.

\subsection{Elastic Modulus and Adhesion at the Interphase}

Figure 5.2 depicts the height and adhesion images acquired on the composite samples. These large scans $\left(50 \times 50 \mu^{2}\right)$ were acquired to find CNCs through the polymer matrix. Due to the fact that CNCs are embedded in the matrix, the height image (Figure 5.2a) is not able to detect the presence of CNCs. In most cases CNCs (shown by yellow arrows) had almost the same height as the surrounding polymer; therefore not much image contrast appeared in the height map. On the other hand, the difference in adhesion properties of CNCs and polymer matrix provided apparent contrast in the adhesion maps and made it possible to distinguish CNCs from neighboring polymer (Figure 5.2b). In general, throughout the sample, the adhesion of the tip to the polymer matrix $(36 \pm 7.2$ $n N)$ was higher compared to its adhesion to CNCs $(25.6 \pm 4.3 n N)$. The difference can be explained by the repulsive forces that can occur between the negative surface charges on the CNC surface (after sulfuric acid hydrolysis (Habibi et al. 2010)) and the thin oxide layer on the AFM tip.
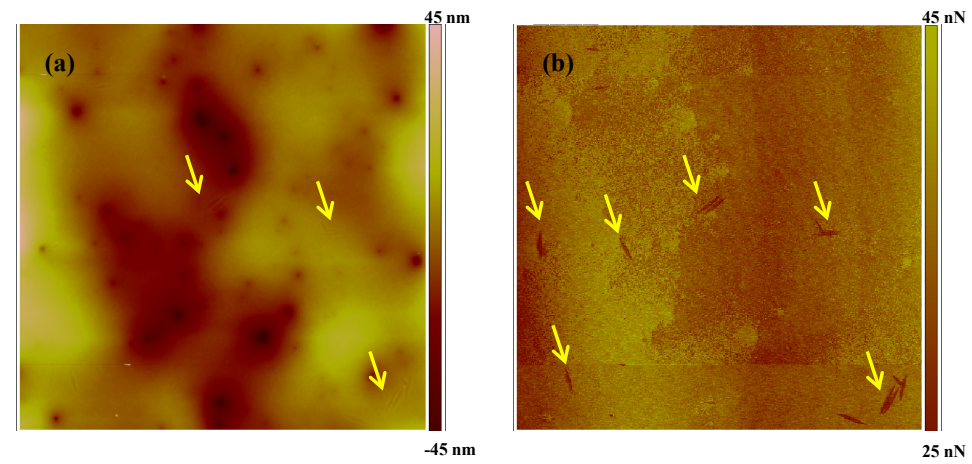

Figure 5.2 (a) Height and (b) adhesion maps of a $50 \times 50 \mu \mathrm{m}^{2}$ region on a PVA $80-\mathrm{CNC}$ 10 - PAA 10 sample. The arrows point to CNCs embedded in the polymer matrix. The presence of $\mathrm{CNCs}$ is easily distinguishable from the adhesion image.

Figures 5.3a shows the three-dimensional (3D) height image of a CNC aggregate, where the width gradually changes from one end to another. The corresponding adhesion and elastic modulus maps are shown in Figure 5.3b and c, respectively. The average variation of mechanical properties in the region, identified by the red box, can be seen in Figure 5.3d. The blue and orange curves in Figure 5.3d represent the adhesion and elastic modulus profiles, respectively. According to the elastic modulus profile, the detected mechanical properties are higher in the locations where CNCs exist. In contrast, the 
adhesion properties are lower in the locations of CNCs. This is consistent with the observation made in Figure 5.2b. The scattering of the data observed on the profiles in Figure $5.3 \mathrm{~d}$ can be due to the influence of surface roughness and small indentation depth made at each tap.

The elastic modulus of CNC $(12.8 \pm 3.1 \mathrm{GPa})$ was found to be higher than PVA-PAA matrix $(9.9 \pm 1.1 \mathrm{GPa})$. One should note that the $\mathrm{CNC}$ elastic constants reported here are the average transverse moduli $\left(\mathrm{E}_{\mathrm{T}}\right)$ and are close to the previously reported data (Lahiji et al. 2010; Nakamae et al. 1986; Tashiro and Kobayashi 1991). Tashiro and Kobayashi (1991) calculated the elastic modulus of CNC in two orthogonal directions perpendicular to the $\mathrm{CNC}$ axis $\left(T_{1}\right.$ and $\left.T_{2}\right)$ equal to $E_{T 1}=51$ and $E_{T 2}=57 \mathrm{GPa}$. Lahiji et al. (2010) used nanoindentation in AFM and measured the transverse elastic modulus of cotton CNCs varying between 18 to $50 \mathrm{GPa}$ at $1 \%$ relative humidity. Nakamae et al. (1986) used an Xray diffraction technique and estimated the theoretical transverse elastic modulus of PVA to be $\mathrm{E}_{\mathrm{T}}=6-9 \mathrm{GPa}$.

(a)

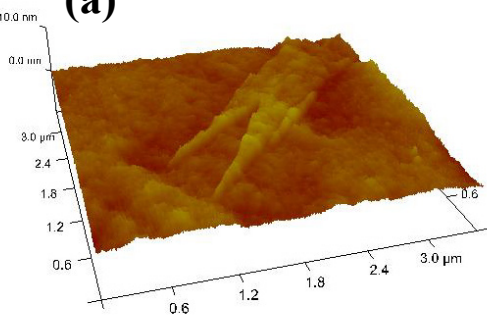

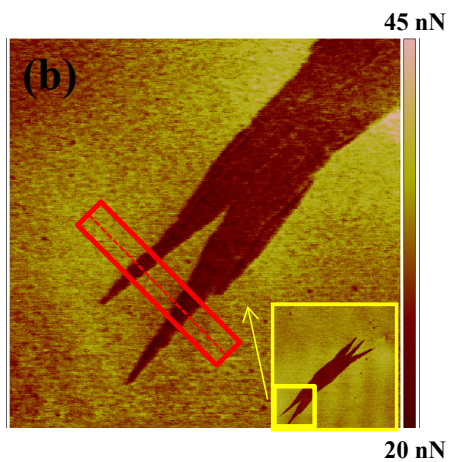

$16 \mathrm{GPa}$

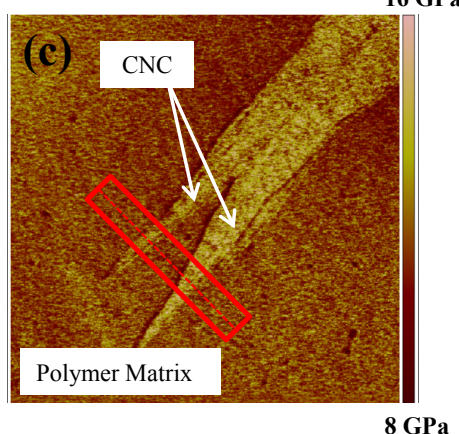

(d)

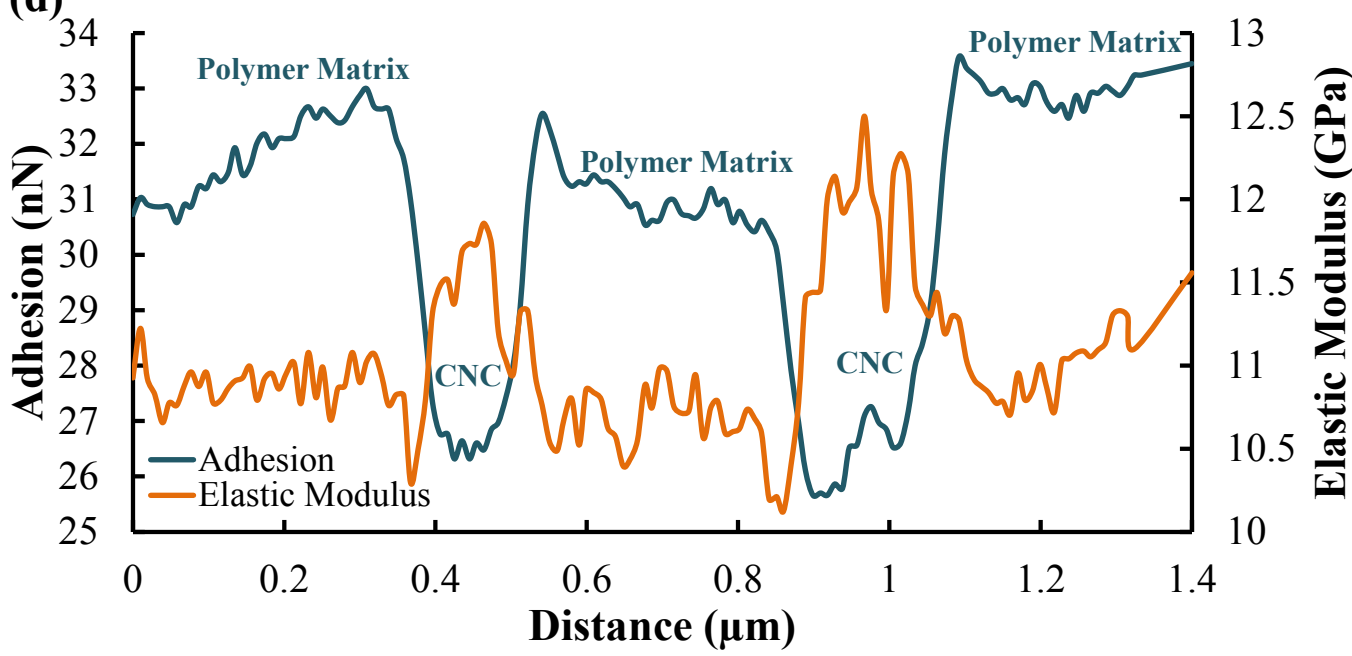

Figure 5.3 3.5 $\times 3.5 \mu \mathrm{m}^{2}$ (a) 3D height, (b) adhesion, (c) elastic modulus maps. Inset in (b) shows the scanning area (PVA 80- CNC 10- PAA 10). (d) The average adhesion and elastic modulus profiles of the area in the red boxes in (b) and (c). Polymer matrix and $\mathrm{CNC}$ regions are marked on the profile. Interphase region is distance that the overlapping in adhesion and modulus profiles is observed. 
Next, the variation in matrix properties as a function of distance from the surface of $\mathrm{CNC}$ was studied. As seen in Figure 5.3, the elastic modulus gradually changed from $\sim 12$ $G P a$ at the CNC interface to $\sim 10.5 \mathrm{GPa}$ at PVA-PAA interface. The distance over which the properties changed is called 'interphase' hereafter. The gradual change in nanomechanical properties of the interphase can be explained by gradient distribution of ester linkages (cross linking density) from the $\mathrm{CNC}$ interface to the polymer matrix. Figure 5.4a shows the schematic of a CNC embedded in a pure PVA matrix. Here, no PAA chains exist in the composite and only PAA molecules surround $\mathrm{CNC}$ via hydrogen bonds with its surface. Figure 5.4b-c show the schematics of a cellulose nanocrystal embedded in PVA-PAA polymer matrix before and after the cross-linking process. In Figure 5.4b (before ester links are formed), there is no covalent bond between PVA, PAA, and CNC. PVA molecules surround CNC and form hydrogen bonds with cellulose crystal as well as each other. On the other hand, not many PAA molecules are present close to the CNC surface. The surface energy between CNC and PVA is lower than the surface energy at CNC-PAA interface and this can be explained by great likelihood of formation of hydrogen bonds between $\mathrm{CNC}$ and PVA as opposed to CNC and PAA. Hyder et al. (2006) have reported that measuring the contact angle for pure PVA films is not possible because water swells the polymer in less than a minute. This shows the hydrophilic nature of PVA and, in other words, its high tendency to form hydrogen bonds. PAA is not very hydrophilic, and does not form hydrogen bonds. For this same reason polar components are usually added to PAA to act as hydrogen bond accepters and to improve the PAA's adhesive properties (Shojaie and Xiaoling 1997). After the composite is heated, ester links are formed between PVA-PAA, PVA-PAA-CNC or CNC-PAA (Figure 5.4c). Here, due to esterification, the number of hydroxyl groups on CNC surface is less than prior to cross-linking. Thus, hydrogen bonding still exists but is less between CNC and PVA. Formation of ester linkages results in the improvement of mechanical property and thermal stability of PVA membranes (Paralikar et al. 2008). Likewise, existence and density of these links in the vicinity of CNC can affect the properties of the interphase.

In order to investigate the effect of ester links that form due to the existence of PAA, the rate of change in adhesion at the interphase was calculated for two composite systems; one with PAA and cross linked (PVA 80- CNC 10- PAA 10, rectangular symbols in Figure 5.5) and one without PAA (PVA 85- CNC 15- PAA 0, triangle symbols in Figure 5.5). For this purpose, the average adhesion profiles were acquired at CNC sites in each sample. Slopes of these curves were calculated at the interphase regions (dashed lines on Figure 5.5). In order to rule out the dependency on CNC size, care was taken to select CNCs with similar diameters. It was found that the rate of change in adhesion at the interphase was $0.08 \pm 0.03 n N . n m^{-1}$ in PVA 85- CNC 15- PAA 0, while this rate was $0.12 \pm 0.02 n N . n m^{-1}$ in PVA 80 - CNC 10- PAA 10 samples. This is equivalent to $\sim 33 \%$ difference in the rate of change in adhesion in these two samples. This difference confirms that the existence of PAA has increased the gradient of mechanical properties of the interphase. 


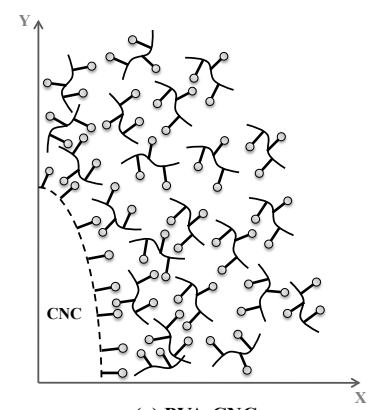

(a) PVA-CNC
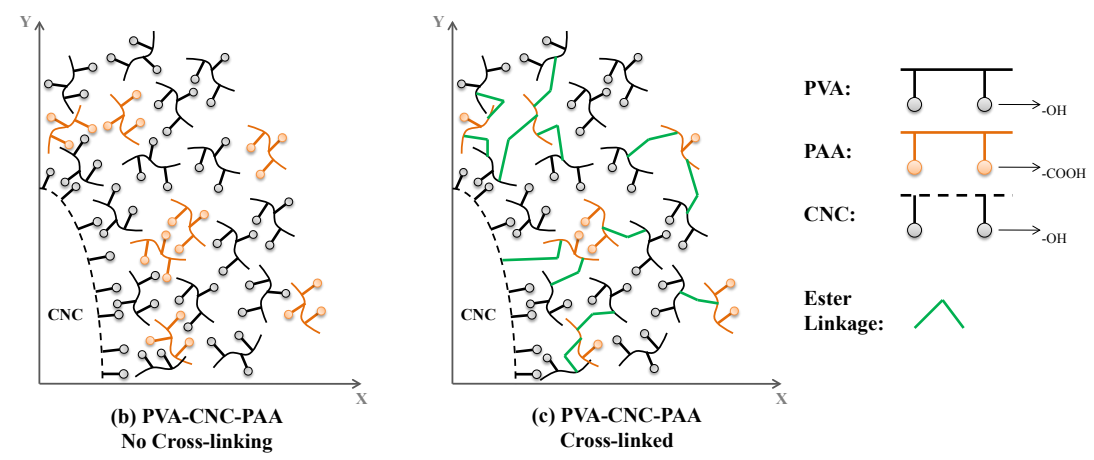

Figure 5.4 Structure models of PVA membranes containing (a) no PAA, (b) PAA and before cross-linking, and (c) PAA and after cross linking. In PVA-CNC membrane, only hydrogen bonds between $\mathrm{CNC}$ and PVA exist at the interphase. In the PVA-CNC-PAA case (before cross-linking), PVA molecules form hydrogen bonds with hydroxyl groups on the CNC surface. Some PAA molecules also exist in the vicinity of CNC. After crosslinking, ester bonds form and the population of these bonds decreases as the distance from $\mathrm{CNC}$ surface increases. (Figure is not to scale)

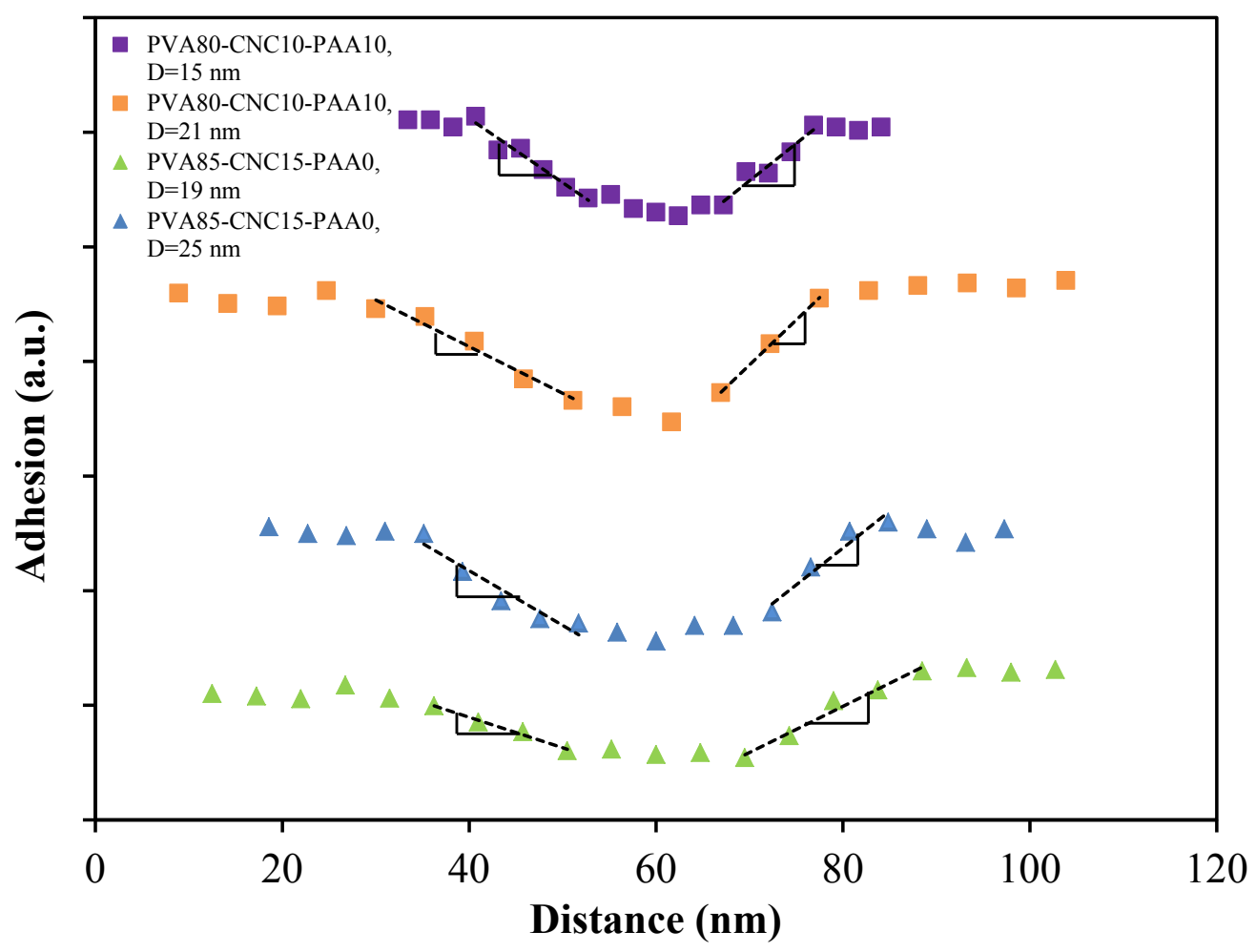

Figure 5.5 Average adhesion profiles (arbitrary unit, a.u.) in PVA 80- CNC 10- PAA 0 (square) and PVA 85- CNC 15- PAA 0 (triangle) composites. Dashed lines were used to measure the rate of change in adhesion at the interphase $\left(\mathrm{nN} \cdot \mathrm{nm}^{-1}\right)$.

Similar to PVA 80- CNC 10- PAA 10 membrane, the existence of gradient in mechanical properties in PVA 85- CNC 15- PAA 0 sample is expected. It was observed 
that the mechanical properties decreased from the CNC surface to the PVA matrix. This can be explained by the gradient in hydrogen bonding from the $\mathrm{CNC}$ surface towards the polymer matrix. As shown in Figure 5.4a, at the surface of CNC, CNC-CNC, PVA-PVA, and PVA-CNC hydrogen bonds coexist. The number of PVA-CNC hydrogen bonds decreases from the surface of CNC into the polymer matrix, where only PVA-PVA bonds are present (Figure 5.4a). The number and nature of these hydrogen bonds have been shown to have substantial effect on the mechanical properties in such molecular materials (Nakamae et al. 1986; Peijs et al. 1995; Tashiro and Kobayashi 1991; Tashiro and Tadokoro 1981).

\subsection{Interphase Thickness and CNC Diameter}

The ability to measure the thickness of interphase in nanocomposites allows one to study the relationship between the interphase size and the size of the reinforcement. Here, in order to study size dependency of interphase on the CNC size, the interphase thickness was measured on both sides for various $\mathrm{CNCs}$ in the composite system. Figure 5.6a depicts an individual $\mathrm{CNC}$ with diameter of $\sim 9 \mathrm{~nm}$, measured from the adhesion image (Figure 5.6b). The adhesion map shows that the mechanical properties of the CNC are fairly uniform along its axial direction. The thickness of interphase was between 11-13 $\mathrm{nm}$ on either sides of this crystal (Figure 5.6c). Using such profiles and the corresponding adhesion maps, the CNC diameters and interphase thickness on either sides of CNCs were recorded. The average interphase thickness was calculated using the size of the interphase on both sides.

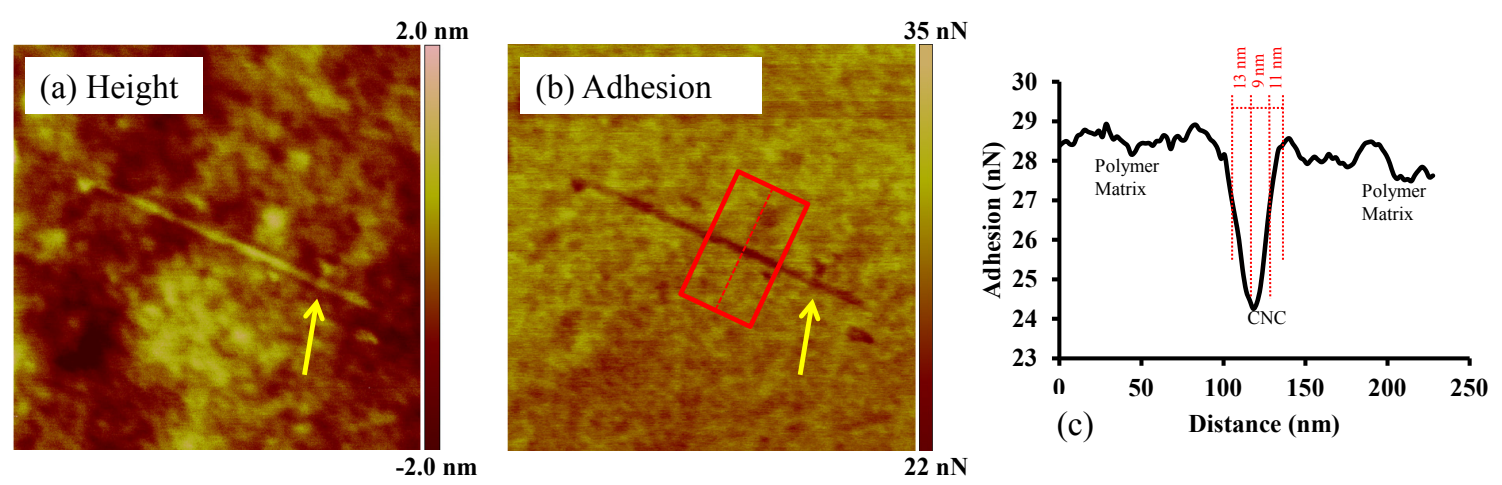

Figure 5.6 $1 \times 1 \mu \mathrm{m}^{2}$ height (a) and adhesion (b) maps on a single CNC. (c) Average adhesion profile of the area shown by red rectangle in adhesion map in (b). Using this profile, $\mathrm{CNC}$ diameter $(9 \mathrm{~nm})$ and average thickness of the interphase were measured.

Figure 5.7 depicts the variation in the average interphase thickness as a function of $\mathrm{CNC}$ size. As seen, the interphase thickness increased with the increase of CNC diameter. The reason for this behavior is not clear at this stage, but one explanation can be given based on the increase of surface area in nanocrystals. Depending on the size of the CNC, the thickness of the interphase varied between 4 to $35 \mathrm{~nm}$. For instance, taking two CNCs with similar aspect ratios (length/diameter $=50$ ) and different diameters of 10 and $30 \mathrm{~nm}$, the surface area, for the $\mathrm{CNC}$ with diameter equal to $30 \mathrm{~nm},\left(142713 \mathrm{~nm}^{2}\right)$ is $\sim$ nine times 
larger than that of the smaller CNC. This larger surface area can give rise to a thicker interphase (Liu and Brinson 2008; Long and Lequeux 2001).

To the authors' knowledge, this is the first nanoscale experimental effort to characterize the size of interphase in CNC-composite systems. Our data can be important to those interested in developing accurate models of interface in nanocomposite systems (Brown et al. 2008; Jancar et al. 2010; Li et al. 2011). Brown et al. (2008) studied the effect of nanoparticle diameter on the thickness of interphase by molecular dynamics. They found that the thickness of interphase remained constant independent of the inclusion size and volume fraction of reinforcement. In another work, Li et al. (2011) utilized a hierarchical multi-interphase model and showed that the volume fraction of the interphase is size-independent. They concluded that the effect of interphase thickness on the reinforcing efficiency of nanomaterials vanishes as length is increased above $40 \mathrm{~nm}$. The present investigation shows that the thickness of the interphase is not constant and should be taken into account in future theoretical frameworks for the interphase consideration and particularly in the CNC-polymer composite systems.

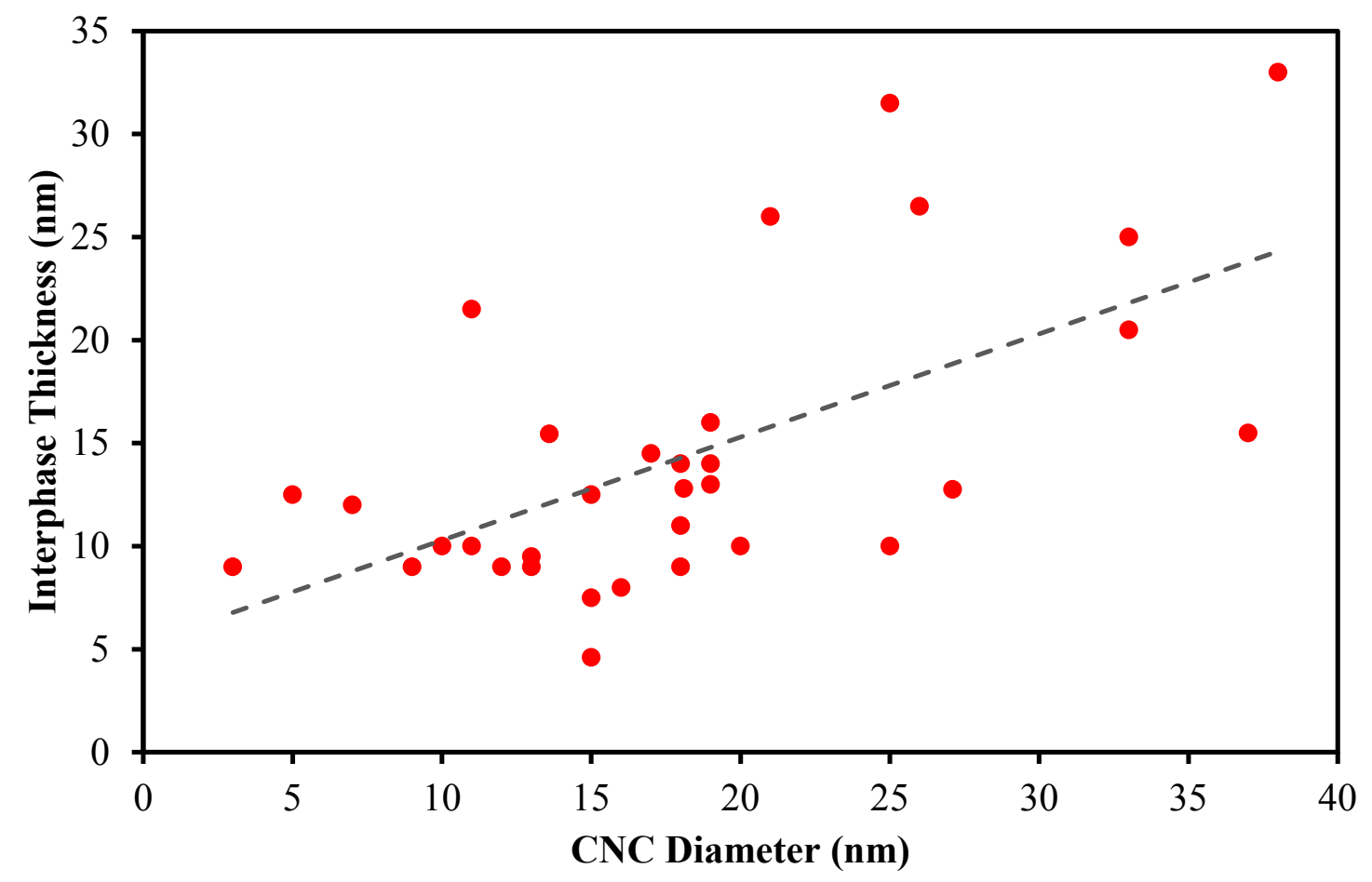

Figure 5.7 Change in interphase thickness with $\mathrm{CNC}$ diameter. Red circles show average interphase thickness of the CNCs. The trend line (dashed gray line) shows an increasing trend in the interphase thickness with the $\mathrm{CNC}$ diameter.

\subsection{Conclusions}

Nanomechanical properties of the interphase (the zone between CNC surface and polymer matrix) were quantitatively characterized in poly (vinyl alcohol) -poly (acrylic acid)-cellulose nanocrystal composites using peak force tapping mode in AFM. As 
opposed to topography images, nanomechanical and adhesion maps enabled the detection of CNCs embedded in the PVA-PAA polymer matrix. The interphase in the composites containing PAA exhibited a higher gradient in adhesive and mechanical properties in comparison to the samples with no PAA. From the polymer matrix to the CNC surface, the average elastic modulus increased from 9.9 to $12.8 \mathrm{GPa}$, and the adhesion to the AFM decreased from 36 to $25.6 \mathrm{nN}$, respectively. The observation of higher gradient in properties of the composites with PAA was related to the increase in the density of ester linkages from the polymer matrix to the $\mathrm{CNC}$ interface. It was also shown that the interphase thickness depended on the $\mathrm{CNC}$ size. Because larger CNCs have higher surface area, the influence of $\mathrm{CNC}$ penetrates deeper in the polymer and results in higher interphase thickness.

\subsection{Acknowledgements}

The authors acknowledge the National Science Foundation (NSF) for the grants No. 0820884 and No. 11000806/1100572 from Division of Materials Research (DMR). Also, thanks Dr. M. C. Frost for constructive discussions on the data. R.S.Y. also thanks the start up fund from Michigan Technological University. 


\section{Size Effects on the Nanomechanical Properties of Cellulose I Nanocrystals ${ }^{5}$}

\subsection{Introduction}

Cellulose is one of the most abundant materials on earth, forming the building block of plants, algae, and some animal structures. Cellulose is a linear polymer, which has several hydroxyl groups on its surface. Existence of these hydroxyl groups results in formation of strong hydrogen bonds between neighboring cellulose molecules that form microfibrils a few hundred nanometers in diameter and several micrometers in length. These fibrils consist of both crystalline and amorphous regions (Beck Candanedo et al. 2005; Bodenson et al. 2006) and can be divided into much smaller features called cellulose nanocrystals (CNCs) via different mechanical (homogenization, grinding, and micro-fluidization (Turbark et al. 1983; Taniguchi and Okamura 1998; Zimmermann et al. 2004)) or chemical treatments (most commonly sulfuric acid hydrolysis (Azizi Samir et al. 2005)).

Similar to other nanomaterials, CNCs have attractive properties such as a high surfaceto-volume ratio, high aspect ratio (length $(L)$ to diameter $(D)$ ratio) and low density $\left(1.566 \mathrm{~g} / \mathrm{cm}^{3}\right.$ (Azizi Samir et al. 2005)), that make them favorable for application in polymer composites. Consequently, there are numerous reports in literature that show the reinforcing characteristics of these crystals in polymers (Favier et al. 1995; Cao et al. 2007; Kvien et al. 2007; Lapa et al. 2007; Auad et al. 2008;). In composite materials, the properties of the interphase is dictated by transverse mechanical properties, size, and surface characteristics of the nano reinforcement (Long and Lequeux 2001; Liu and Brinson 2008). Thus, in order to better understand the role of $\mathrm{CNC}$ in polymer composites and increase its potential to be used in industrial scales, it is necessary to study the CNC transverse elastic modulus and its dependence on CNC size.

Although the axial (along the axis) elastic modulus of CNCs from different sources has been under investigation for many years (Meyer and Lotmar 1936; Sakurada et al. 1962; ; Sakurada et al. 1964; Jaswon et al. 1968; Tashiro and Kobayashi 1985; Kroon et al. 1986; Matsuo et al. 1990; Tashiro and Kobayashi 1991; Nishino et al. 1995; Guhados et al. 2005; Tanaka and Iwata 2006; Cheng and Wang 2008; Iwamoto et al. 2009), there are not many reports on the transverse elastic modulus (perpendicular to major axis, $E_{t}$ ) of these materials (Jaswon et al. 1968; Tashiro and Kobayashi 1991; Lahiji et al. 2010).

In 1968, using theoretical modeling of cellulose I, Jaswon et al.(1968) estimated values of 76 and 51 and $57 \mathrm{GPa}$ for axial and two perpendicular transverse directions, respectively. Similarly, in 1991, Tashiro and Kobayashi (1991) predicated elastic moduli

\footnotetext{
${ }^{5}$ The material contained in this chapter has been accepted for publication in Journal of Material Research, Focus Issue (February 2012). See Appendix C for proper documentations.
} 
of 167 and 11 and $50 \mathrm{GPa}$ for axial and two transverse elastic moduli of cellulose crystals, respectively. This elastic modulus anisotropy has been associated with the cellulose chain anisotropy in CNC structure. Just recently, the first experimental data on characterization of the transverse elastic moduli of CNCs using atomic force microscopy (AFM) was published (Lahiji et al. 2010). Lahiji et al. used AFM measurements and a physics-based model and calculated $E_{t}=18-50 \mathrm{GPa}$ for wood-derived CNCs. The molecular structure of a low density polymer such as cellulose is the major reason for the high elastic modulus observed in CNCs. There are multiple hydroxyl groups on the cellulose chain. These can make inter- and intra-molecular hydrogen bonds, which have a key role in the mechanics of this material (Meyer and Lotmar 1936; Lyons 1959; Treloar 1960; Mann and Roldan-Gonzalez 1962; Sakuradda et al. 1962).

In the current study, peak force tapping mode (PF-TM), a relatively new technique in atomic force microscope (AFM), was used to study the nanomechanical properties (AFM tip-CNC adhesion force) of single CNCs. The structural properties of crystals derived from two different sources (wood and cotton) were compared. Moreover, transverse elastic moduli of these CNCs were studied using nanoindentation module in AFM in combination with both analytical contact mechanics modeling and finite element analysis. Therefore, the effect of $\mathrm{CNC}$ source was investigated on their transverse elastic modulus as well as the accuracy of different approaches for calculation of $E_{t}$ could be examined by comparing the results with theoretically predicted values. Finally, the variation of adhesion forces and $E_{t}$ along the $\mathrm{CNC}$ axis and their dependency on $\mathrm{CNC}$ source and diameter were studied.

\subsection{Experimental Procedures}

\subsubsection{Materials}

CNCs were prepared by acid hydrolysis of cellulose as described by Beck-Candanedo et al. (2005). Filter paper (Whatman \#1, Clifton, NJ) and commercially available microcrystalline cellulose (MCC) were used as the source of cotton-CNCs and wood$\mathrm{CNCs}$, respectively. For this purpose cellulose source in powder was stirred with $65 \%$ $\mathrm{H}_{2} \mathrm{SO}_{4}(v / v)$ for $50 \mathrm{~min}$, at $45^{\circ} \mathrm{C}$. The cellulose-to-acid ratio was $1: 10 \mathrm{~g} / \mathrm{ml}$. After the mixture was centrifuged five times, it was subjected to ultrasonication (Branson Sonifier, Danbury, CT) for $15 \mathrm{~min}$, in order to break agglomerations and evenly disperse CNCs in water. To remove remaining salts, ultra filtration was done until the conductivity was $<10$ $\mu S / c$, (Ultrasette tangential flow UF device, pore size $=50 \mathrm{~nm}$, Pall Corp., Ann Harbor, MI). Finally, an aqueous suspension of $1 \% \mathrm{CNC}$ was obtained by concentrating the dispersion of CNCs in a Rotavaporizer R110 (Buchi, Flawil, Switzerland).

The samples used for AFM imaging were prepared by placing a few drops of CNC dilute solution onto a $1.5 \times 1.5 \mathrm{~cm}^{2}$ of freshly cleaved mica. In order to get a better dispersion of crystals on the mica surface, samples were rinsed with de-ionized water and blown dry before the droplets were dry. After rinsing and blow-drying, the CNCs remaining on the mica surface were strongly adhered to the mica. 


\subsubsection{AFM Measurements}

Bruker's Dimension (C) Icon $($ C AFM was used for imaging and nanoindentation tests in this work. The combined nanoindentation and AFM capabilities made it possible to find the sample of interest by imaging and then to perform the nanoindentation. AFM images were acquired using peak-force tapping mode (PF-TM) (Pittenger et al. 2009). In this technique, the cantilever is not resonated. Here, using the main $Z$ piezoelement, the vertical motion of the cantilever is oscillated below its resonant frequency and for each individual tap, nanoscale material property and peak force is collected (one force curve is collected for each pixel on the image). Each force-separation $(F-h)$ curve is then analyzed to generate material property maps with the same resolution as the height image. These include Derjaguin-Muller-Toporov (DMT) modulus (Derjaguin et al. 1975), adhesion, and deformation maps.

A silicon cantilever with a nominal spring constant of $20-80 \mathrm{~N} / \mathrm{m}$ and tip radius of 5$12 \mathrm{~nm}$ was used for both imaging and indentation studies. The deflection sensitivity of the cantilever was measured by acquiring a force curve on a hard sapphire surface. This was repeated at least five positions to make sure the value was consistent $(85.38 \mathrm{~N} / \mathrm{m})$. The spring constant was acquired equal to $24.7 \mathrm{~N} / \mathrm{m}$ using the Sedar method (Ohler 2009). The tip radius was measured using the relative method. This method involves scanning a standard sample (Poly Styrene), with a known elastic modulus, and altering the tip radius until the expected value $(2.7 \mathrm{GPa})$ was obtained in the DMT modulus channel (tip radius for $2 \mathrm{~nm}$ deformation was equal to $5 \mathrm{~nm}$ ). Finally, the Poisson's ratio value of 0.3 was used for all samples. These settings were kept constant for all the subsequent measurements.

AFM images consisted of $512 \times 512$ pixels. Scanning rate was changed according to the scan size, and was $<1 \mathrm{~Hz}$ at all times. The peak force was set such that the resultant average deformation in each scan line was not more than $2 \mathrm{~nm}$. Other scanning parameters, such as integral and proportional gains, were automatically set by the Nanoscope software.

Nanoindetation in AFM: A raw AFM force curve is a plot of the position of the piezo ( $z$ in $\mathrm{nm}$ ) that runs the AFM versus the output voltage of the photo-detector $(\Delta$, in $V$ ). In order to convert this to a more common force-displacement or force-indentation height $(F-h)$ curve, deflection sensitivity is used to convert the units of $\Delta$ to $n m$. Force $(F)$ is then calculated in $n N$, by multiplying the deflection of the cantilever by its spring constant, $k$ $(F=k \Delta)$. Indentation height ( $h$, value of deformation) is calculated by subtracting the cantilever deflection from the vertical piezo position $(z)$. For each sample (cotton- and wood-CNCs), at least ten measurements were made. Additionally, several measurements were conducted along the axis of each $\mathrm{CNC}$ to investigate the variation of $E_{t}$. Maximum indentation of $2 \mathrm{~nm}$ was applied at each point and the corresponding force was measured. Nanoindetation frequency (which is related to the loading rate) was $1 \mathrm{~Hz}$. 


\subsubsection{Calculation of CNC Transverse Elastic Modulus}

After $F$ - $h$ curves are acquired in AFM, they can be fitted by several models to estimate the transverse elastic modulus of CNCs $\left(E_{t}\right)$. Here, two different approaches namely analytical continuum contact mechanics modeling and finite element analysis (FEA) were used and the results were compared to theoretical estimated values for $E_{t}$.

Analytical Contact Mechanics: Various contact mechanics models exist for calculating the elastic modulus from AFM nanoindentation data. These include Hertz (Hertz 1882), Johnson-Kendall-Roberts (Johnson et al. 1971), and Derjaguin-Muller-Toporov (Derjaguin et al. 1975). Hertz model assumes that the contact is initialized at a single point and deformation in the indentation area is perfectly elastic. Moreover, little or no adhesion exists between the tip and the sample (in comparison to the applied load), and the spherical tip end and planar film with infinite thickness. The Hertz model has often been applied to the loading curves from AFM nanoindentation experiments by compressing a polymer material between AFM tip and planar substrates (Domke and Radmacher 1998; Tan et al. 2004)). Based on the Hertz model, contact radius (a) and sample deformation or indentation depth $(h)$ are:

$$
\begin{gathered}
a=\sqrt[3]{\frac{R F}{E_{\text {red }}}} \\
h=\frac{a^{2}}{R}
\end{gathered}
$$

where, $R, F$ and $E_{\text {red }}$ are tip radius, applied force, and reduced elastic modulus, respectively. Combining Equations 6.1 and 6.2 give Equation 6.3, which relates the indentation depth to the applied force:

$$
F=E_{r e d} \sqrt[3]{h} \sqrt{R}
$$

where $v_{s}$ and $v_{\text {tip }}$ are the Poisson's ratio of the sample and the tip respectively:

$$
E_{\text {red }}=\left(\frac{1-v_{s}^{2}}{E}+\frac{1-v_{t i p}^{2}}{E_{\text {tip }}}\right)^{-1}
$$

By assuming an infinite elastic modulus for the tip $\left(E_{t i p}\right), E_{\text {red }}$ is equal to the Young's modulus of the sample $(E)$ with a good approximation. In this work, the Hertz model (Equation 6.3) was applied to $F-h$ curves, and the resultant elastic moduli were compared to the elastic moduli obtained from FEA. Hertz model has been used in similar cases, where one-dimensional materials (such as multi walled carbon nanotubes (Palaci et al. 2005) and viral nanotubes (Zhao et al. 2008)) were placed on a substrate and indented by an AFM tip.

Application of the Hertz model is valid only if there is no adhesion between the tip and the samples. To satisfy this condition, relatively high spring constant cantilevers were selected, which helped to minimize the adhesion forces. Another important assumption in the Hertz model is that the contact radius $(a)$ should be small in comparison to the tip 
radius $(R)$. This assumption is satisfied due to the small indentation depths $(2 \mathrm{~nm})$ in the current study (Chizhik et al. 1998; Tsukruk et al. 1998).

It is worth mentioning that, in many cases use of Oliver-Pharr method has been proposed for analysis of nanoindentation data (Feng et al. 2006). Although this method is very useful, it has been shown by several researchers 43 that application of this method is not appropriate for polymer materials due to their viscoelastic.

Finite Element Analysis: The elastic modulus was also estimated by tuning the theoretical elastic modulus value in the FEA model to match the theoretical prediction with experimental data. A 3D quarter symmetry model containing approximately 13,000 elements was created in ANSYS (ANSYS Inc., Houston, TX). As shown in Figure 6.1, a $\mathrm{CNC}$ was modeled as a solid cylinder with a $7 \mathrm{~nm}$ diameter; the AFM tip was modeled as a rigid sphere with $20 \mathrm{~nm}$ radius; and the substrate was modeled as a rigid block with properties of mica. It was assumed that the CNC, AFM tip and substrate have isotropic mechanical properties. The AFM tip radius is larger than what was measured during calibration steps in order to account for the tip blunting.

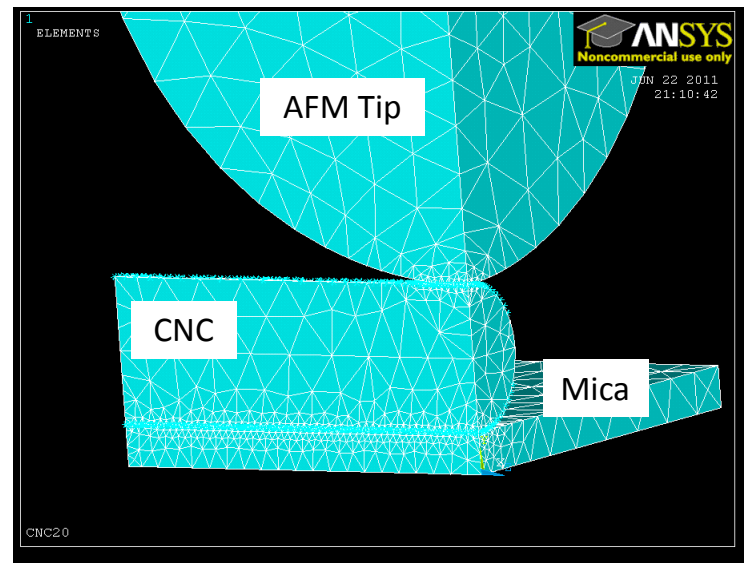

(a)

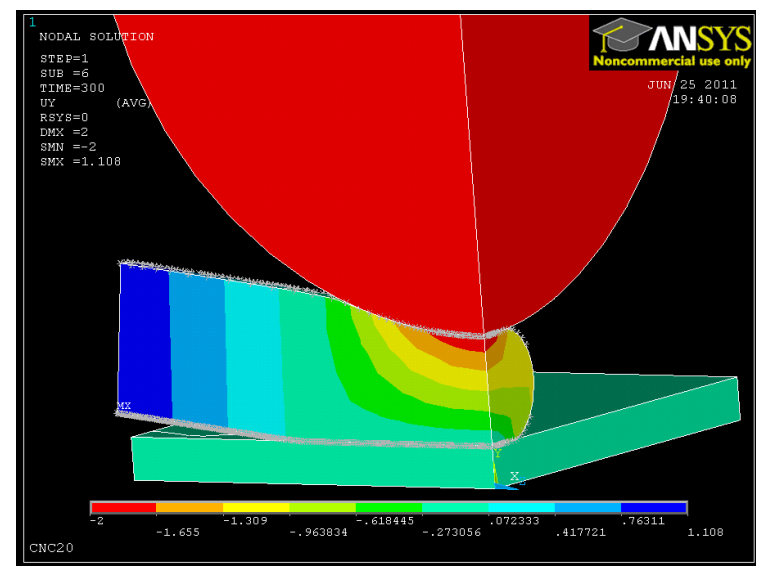

(b)

Figure 6.1 3D quarter symmetry model in FEA; (a) shows the elements and finer mesh at the contact regions at CNC-AFM tip and CNC-Mica interfaces, and (b) shows the deformation along the vertical axis at maximum indentation. The color contour in (b) represents the amount of deformation in the model, which varied from $-2 \mathrm{~nm}$ (red) to 1.1 $n m$ (dark blue).

Ten-node and 3D tetrahedral elements were selected for this model and the mesh was refined where CNC contacted the substrate or the AFM tip. Two contact pairs, one between $\mathrm{CNC}$ and the mica and one between $\mathrm{CNC}$ and the AFM tip, were defined. In order to generate a stable model, vertical displacements equal to indentation height, were applied to the nodes in the AFM tip. Additionally, all other degrees of freedom of the substrate were considered to be fixed. The deformation was applied to $\mathrm{CNC}$ by increasing the vertical displacement of the tip gradually until it reached the maximum value of -2 $\mathrm{nm}$. Figure $6.1 \mathrm{~b}$ represents the deformed shape of $\mathrm{CNC}$ and the amount of deformation in $\mathrm{CNC}$, AFM tip, and mica. After the model was solved, the force was plotted as a function 
of displacement (indentation height) at the contact area between CNC and the AFM tip. This was repeated multiple times, while all parameters were kept constant except for the elastic modulus of the CNC.

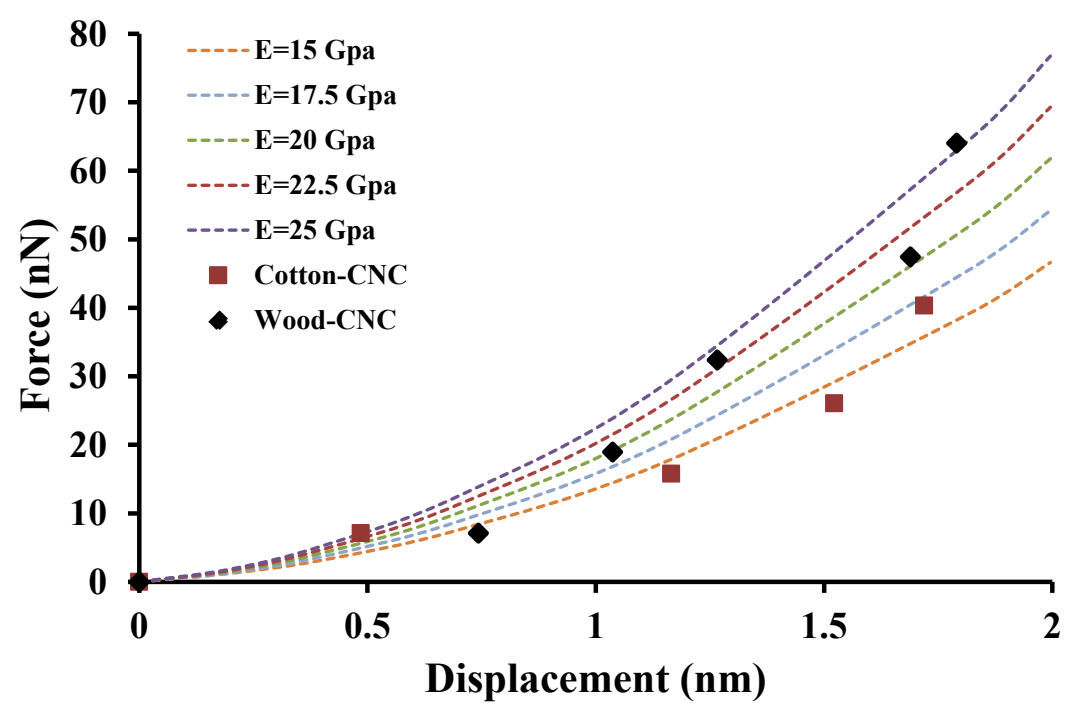

Figure 6.2 $F$ - $h$ curves on a wood-CNC with diameter equal to $3.1 \mathrm{~nm}$ (black diamonds) and cotton-CNC with diameter equal to $6.4 \mathrm{~nm}$ (red squares) along with fit curves with varying elastic moduli obtained from FEA.

Figure 6.2 shows two representative $F$ - $h$ curves on a wood-CNC (diameter equal to $3.1 \mathrm{~nm}$ ) and a cotton-CNC (diameter equal to $6.4 \mathrm{~nm}$ ). It also includes several curves, with varying elastic modulus, obtained from FEA. Using this approach, the theoretical curve that could fit the experimental data with the least error was obtained for each AFM $F-h$ curve. It is worth mentioning that the FEA model was solved with the assumption of perfect contact between $\mathrm{CNC}$ and the tip and at the central point on the CNC. For this reason, only $F$ - $h$ curves that were measured along the center of $\mathrm{CNCs}$ were selected for these calculations.

\subsection{Results and Discussions}

Figure 6.3a-d depicts the topography and adhesion maps of representative wood-CNC (Figure 6.3a-b) and cotton-CNC (Figure 6.3c-d) obtained using PF-TM. Graphs in Figure $6.3 \mathrm{e}$ show the change in CNCs' height along their axes. These graphs were acquired by drawing a profile line on the height images (as shown in Figure 6.3a and c). 


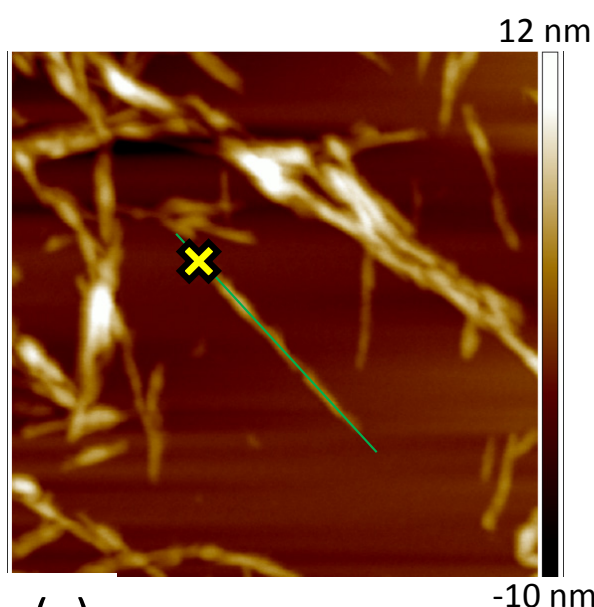

(a)

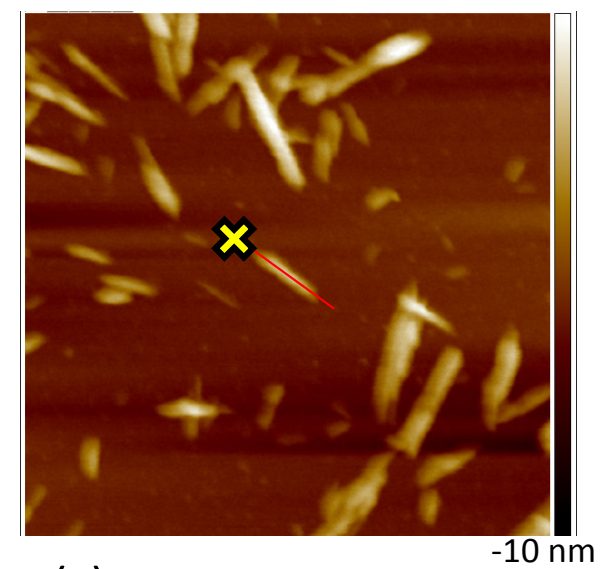

(c)

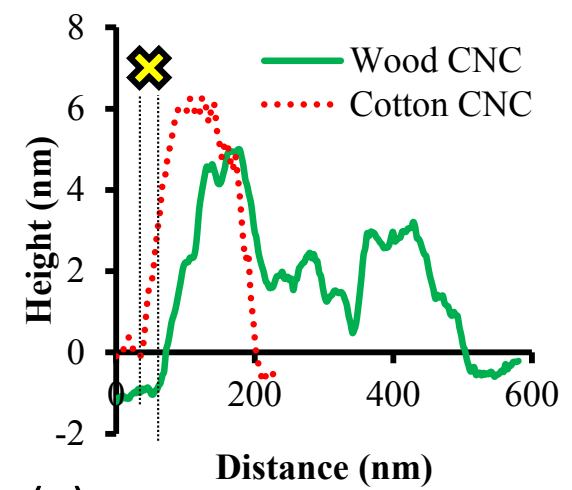

(e)

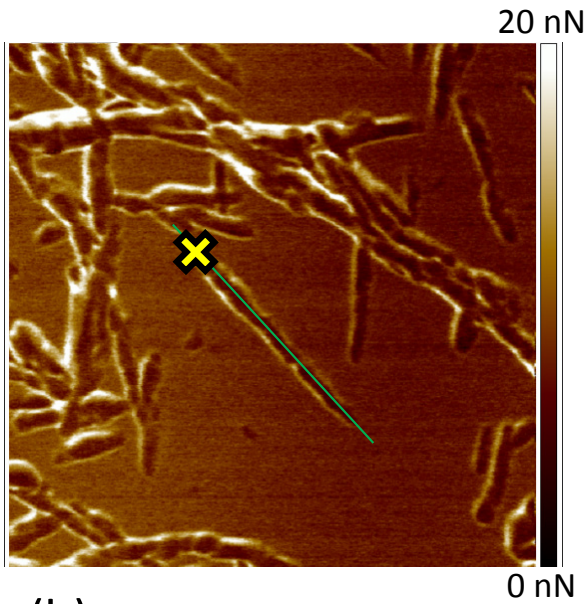

(b)

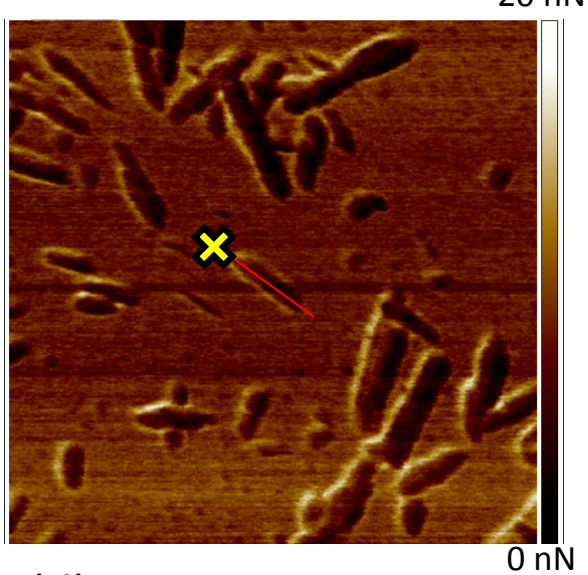

(d)

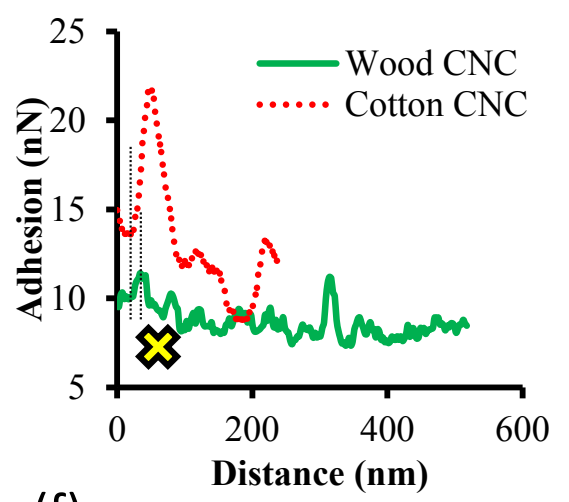

(f)

Figure 6.3 $1 \times 1 \mu m^{2}(\mathrm{a}, \mathrm{c})$ topography and (b, d) adhesion maps of (a, b) wood and (c, d) cotton CNCs. The topography (e) and adhesion (f) profiles were obtained along the lines shown on AFM maps. Dotted red lines and solid green lines correspond to cotton- and wood-CNCs, respectively. Yellow crosses show the direction of the profile lines. 
As summarized in Table 6.1, wood-CNCs were longer $(310 \pm 45 \mathrm{~nm})$ and had smaller diameter $(4.2 \pm 1.2 \mathrm{~nm})$ in comparison to the cotton-CNCs (length and diameter were 166 \pm 34 and $5.9 \pm 1 \mathrm{~nm}$, respectively). These dimensions correlate well with previously published reports (Habibi et al. 2010) and show that the acid hydrolysis of commercially available MCC resulted in higher aspect ratio cellulose nanocrystals. Usually, acid hydrolysis of native cellulose causes a reduction in the degree of polymerization to the so called level-off degree of polymerization (LODP) (Battista et al. 1956). LODP has been shown to correlate well with the periodic crystal sizes along cellulose chains and, consequently, the length of CNCs after acid hydrolysis (Yachi et al. 1983; Habibi et al. 2010). In addition, the diameter of a CNC along its axis was not constant and typically underwent a 2-3 $\mathrm{nm}$ change. This is also consistent with previously reported data (Azizi Samir et al. 2005; Bodenson et al. 2006).

Table 6.1

Summary of the AFM measurements on individual CNCs

\begin{tabular}{|c|c|c|c|c|c|c|}
\hline \multirow{2}{*}{$\begin{array}{l}\text { CNC } \\
\text { Type }\end{array}$} & \multirow{2}{*}{$\begin{array}{c}\text { Diameter } \\
\text { (nm) }\end{array}$} & \multirow{2}{*}{$\begin{array}{c}\text { Length } \\
\text { (nm) }\end{array}$} & \multirow{2}{*}{$\begin{array}{c}\text { Average } \\
\text { Aspect } \\
\text { Ratio } \\
\text { (nm) }\end{array}$} & \multirow{2}{*}{$\begin{array}{c}\text { Adhesion } \\
\text { (nN) }\end{array}$} & \multicolumn{2}{|c|}{$\begin{array}{c}\text { Transverse Elastic } \\
\text { Modulus } \\
\text { (GPa) }\end{array}$} \\
\hline & & & & & $\begin{array}{l}\text { Hertz } \\
\text { Model }\end{array}$ & $\begin{array}{c}\text { Finite } \\
\text { Element } \\
\text { Analysis }\end{array}$ \\
\hline Wood & $4.2 \pm 1.2$ & $310 \pm 45$ & 74 & $11.5 \pm 1.2$ & $4.9 \pm 1.3$ & $24.8 \pm 7.0$ \\
\hline Cotton & $5.9 \pm 1$ & $166 \pm 34$ & 28.7 & $9.2 \pm 0.8$ & $4.8 \pm 1.4$ & $17.7 \pm 5.0$ \\
\hline
\end{tabular}

The adhesion maps demonstrate the amount of adhesion force $(n N)$ between the AFM tip and the sample. For the measurements performed on cotton CNC samples, the adhesion was $9.2 \pm 0.8$ and $10.5 \pm 1.1 n N$ on $\mathrm{CNC}$ and mica, respectively. For the measurements performed on wood-CNC samples, the adhesion was $11.5 \pm 1.2$ and $13.3 \pm$ $2.1 n N$ on $\mathrm{CNC}$ and mica, respectively. Here, several mechanisms can result in the contrast between mica and $\mathrm{CNC}$ including meniscus, contact geometry, and surface energy effects, as well as nanomechanical properties. In the current study, since the experiments were performed at ambient relative humidity, the meniscus effect (caused by water condensing at the contact region) can have a relatively high effect on the adhesion forces between AFM tip and CNC or mica. On the other hand, the elastic modulus of mica (around $170 \mathrm{GPa}$ (McNeil and Grimsditch 1993)) is much higher than the expected transverse elastic moduli for CNCs (11-57 GPa (Jaswon et al. 1968; Tashiro and Kobayashi 1991)). As a result, the amount of deformation generated by the same AFM tip force will be much less on a mica surface compared to CNC. Thus, the AFM tipsample contact region is smaller on mica. Thus, less force will be needed to separate the tip from the surface. This means that, besides the differences in surface energy and contact geometry (Lahiji et al. 2010), the difference in hydrophilicity and mechanical properties of $\mathrm{CNC}$ and mica can result in the contrast observed in the adhesion images. 
In addition, the adhesion forces measured on cotton-CNC samples were higher compared to wood-CNCs (Figure 6.3f). Since both cotton and wood samples were prepared using similar acid hydrolysis procedures and AFM PF-TM experiments were conducted in similar conditions, among the mechanisms mentioned above, the difference in mechanical properties of these crystals along their axes and with each other appears to be the only reason for such observations. This will be discussed further in the subsequent paragraphs.

Table 6.1 also presents the $E_{t}$ calculations completed using two different methods (Hertz contact mechanics and finite element). In order to follow the assumptions as close as possible, only experimental data obtained on the center line of CNCs was used for curve fitting. One should keep in mind that relating $E_{t}$ measured in the current study to a specific crystallographic orientation in CNCs $\left(E_{t 1}\right.$ and $\left.E_{t 2}\right)$ is difficult because such orientation is not known when CNCs are placed on the mica surface. The fact that these orientations also depend on the shape of CNC after acid hydrolysis (that results in different planes exposed on the surface) makes this task even more challenging. When considering the values reported here, one should also remember that both Hertz and FEA models are for isotropic materials and were used to calculate an anisotropic material property here.

In average, the $E_{t}$ values calculated using the Hertz model were lower in comparison to the $E_{t}$ values calculated by the FEA method. The $E_{t}$ values calculated using FEA (24.8 \pm 7.0 and $17.7 \pm 5.0 \mathrm{GPa}$ for wood- and cotton-CNCs, respectively) were much closer to the previously reported experimental data (Lahiji et al. 2010) and theoretically estimated values (Jaswon et al. 1968; Tashiro and Kobayashi 1991). This is because the Hertz model is not accurate at low forces and when adhesion forces are present. In addition, as compared to FEA (spherical-rod contact geometry), the real contact geometry in AFM experiments is reduced to a simpler spherical-plane geometry in the Hertz model. Also, the effect of the substrate is taken into consideration in the FEA model while it is neglected in the Hertz model. All of these factors have a substantial effect on the $E_{t}$ calculations. For this reason, further analyses were conducted only on calculations based on AFM data and FEA models.

At this point it is worth mentioning the mechanical properties of $\mathrm{CNC}$ and mica are known to be anisotropic. AFM force-displacement curves in this study were obtained by applying a vertical force in direction of $\mathrm{CNC}$ diameter (transverse direction). As a result the AFM $F$ - $h$ curves have captured the natural properties of $\mathrm{CNCs}$ and include the anisotropicity. Next the FEA model was tuned to best fit this experimental data. Therefore, the values predicted by the FEA model are transverse elastic moduli of CNC samples, which as discussed above, match perfectly by previously reported values (experimental and theoretical). On the other hand, the elastic modulus of CNCs in axial direction (Meyer and Lotmar 1936; Sakurada et al. 1962; Sakurada et al. 1964; Jaswon et al. 1968; Tashiro and Kobayashi 1985; Kroon et al. 1986; Matsuo et al. 1990; Tashiro and Kobayashi 1991; Nishino et al. 1995; Guhados et al. 2005; Tanaka and Iwata 2006; Cheng and Wang 2008; Iwamoto et al. 2009; Lahiji et al. 2010) is different and much higher than the ones reported here. 
Note that the scatter in $E_{t}$ (shown by the standard deviations in Table 6.1) can have several reasons, including: (i) Indentation location uncertainty: Not all the times did the AFM tip land on the highest point on $\mathrm{CNC}$ axis, and this can have great influence on the $F-h$ curves obtained. (ii) Change of tip radius with its roughness: If the roughness of the tip changes (due to inelastic deformations of tip asperities that occur during indentation), the effective tip radius that forms the indentation changes, which can result in uncertainty in load measurements.

Another interesting observation is that CNCs obtained from acid hydrolysis of wood have higher elastic moduli compared to those obtained from cotton (Table 6.1). The reason for such observation can be explained by the difference in crystallinity of these CNCs. It has been shown that wood-based cellulose has a higher degree of crystallinity (77\%) compared to cotton cellulose (71\%) (Ioelovich et al. 2010). On the other hand, the dependence of elastic modulus on the degree of crystallinity has been modeled by considering the samples as a composite consisting of crystalline and amorphous domains. These crystals can be either in a parallel or series arrangement (Eichhorn and Young 2001; Harris 1999), and the elastic moduli ( $E_{\text {parallel }}$ and $\left.E_{\text {series }}\right)$ can be calculated based on equations listed below:

$$
\begin{gathered}
E_{\text {parallel }}=E_{c} V_{c}+E_{a}\left(1-V_{c}\right) \\
\frac{1}{E_{\text {series }}}=\frac{V_{c}}{E_{c}}+\frac{1-V_{c}}{E_{a}}
\end{gathered}
$$

where $E_{c}$ and $E_{a}$ are elastic moduli of the crystalline and amorphous regions and $V_{c}$ is the volume fraction of crystals in the material. Using either of the equations, it is clear that the elastic modulus increases with degree of crystallinity, thus it is expected that wood-CNCs have higher $E_{t}$ compared to cotton-CNCs.

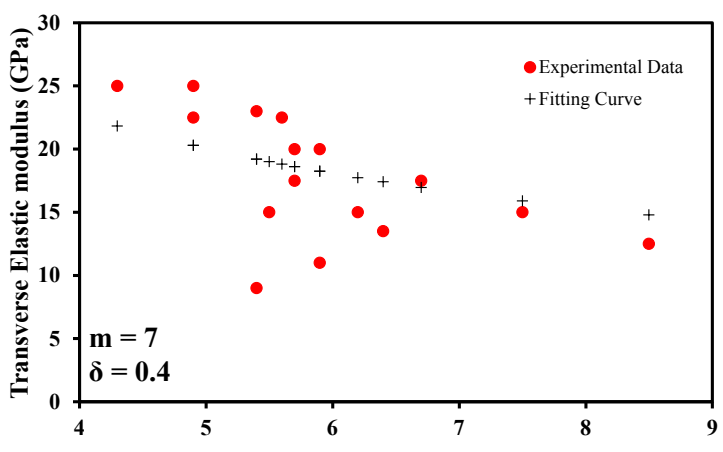

(a) Cotton-CNC

Diameter (nm)

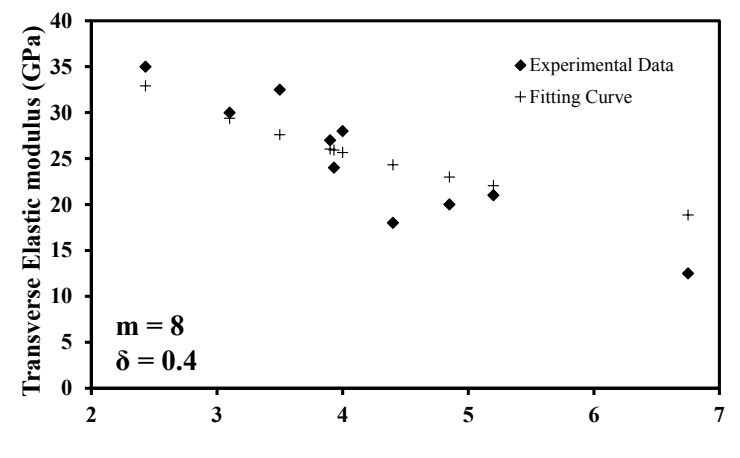

(b) Wood-CNC

Diameter (nm)

Figure 6.4 Change in $E_{t}$ (Calculated using FEA) with the CNC diameter (a) cotton-CNCs (red circles and black crosses show the experimental data and the fitted curve, respectively) and (b) wood-CNCs (black diamonds and black crosses show the experimental data and the fitted curve, respectively). The fitted curves clearly show that as the CNC diameter decreases, $E_{t}$ increases. $m$ and $\delta$ resulted from fitting are shown on the graphs. 
In order to study the size-scale effect on the mechanical properties of these crystals, the transverse elastic modulus was plotted against the diameter of various CNCs (Figure 6.4). Black diamonds and red circles in Figure 6.4 correspond to wood- and cottonCNCs, respectively. In general, wood-CNCs had higher transverse elastic moduli. More importantly, for both CNCs, the elastic modulus increases as the diameter decreases (size-scale effect). Similar behavior was observed on the adhesion force measurements.

To the best of authors' knowledge, size-scale effect on mechanical properties of CNCs has never been reported for cellulose nanocrystals. Several reasons can be behind such behavior in CNCs. Firstly, it can be correlated to the crystalline structures of CNCs and their relationship with the CNC diameter. Back in 1984, Atalla and VanderHart (Atalla and VanderHart 1984) identified two allomorphs of native cellulose $I_{(}\left(\mathrm{I}_{\alpha}\right.$ and $\left.\mathrm{I}_{\beta}\right)$ using nuclear magnetic resonance (NMR) spectroscopy. The major difference between these two structures is how the consecutive hydrogen-bonded planes are arranged on top of each other. While $\mathrm{I}_{\alpha}$ has one-chain triclinic unit cells, $\mathrm{I}_{\beta}$ consists of two-chain monoclinic unit cells. Both allomorphs are present in most kinds of natural cellulose, but $I_{\alpha}$ is dominant in bacterial and algae cellulose and $\mathrm{I}_{\beta}$ is dominant in higher plants such as cotton and wood (Roman and Winter 2006). One other difference between these two allomorphs is their chain stiffness. At the equilibrium $c$-spacing and when hydrogen bonding is present, it has been shown that the chain stiffness is always higher for $\mathrm{I}_{\alpha}(136-$ 149 GPa (Aabloo and French 1994; Nishiyama et al. 2003)) compared to $I_{\beta}$ (109-116 GPa (Finkenstadt and Millane 1998; Nishiyama et al. 2002)).

As the CNC diameter decreases, its surface-to-volume ratio increases. Molecular chains at the surface layers should, in principal, be at a higher energy level compared to those at the core of CNC. Thermodynamically, there will be more likelihood for a metastable $\mathrm{I}_{\alpha}$ crystalline phase to exist on the CNC surface. In fact, $\mathrm{I}_{\alpha}$ has higher potential energy and lower density compared to $I_{\beta}$ (Nishiyama et al. 2002; Kovalenko 2010). Dominance of $\mathrm{I}_{\alpha}$ allomorph has been previously shown on the surface of cellulose crystals (Baker et al. 2000; Malm et al. 2010). Thus, with decrease in diameter, $I_{\alpha} / I_{\beta}$ ratio can increase in $\mathrm{CNCs}$. Since $\mathrm{I}_{\alpha}$ has a higher elastic modulus compared to $\mathrm{I}_{\beta}$, smaller $\mathrm{CNCs}$ that have larger amount of $\mathrm{I}_{\alpha}$ are expected to have higher elastic moduli.

To further quantify this hypothesis, the experimental data was fitted to a core-shell model (Zheng et al. 2010) as shown in Figure 6.4. According to this model, CNC (with diameter $D$ ) is composed of a core and a shell (thickness of the shell is equal to $\delta$ ). The elastic modulus of the whole $\mathrm{CNC}(E)$ can be correlated to the elastic moduli of the core $\left(E_{c}\right)$ and the surface $\left(E_{s}\right)$ :

$$
E=E_{c}+E_{c}(m-1)\left[1-(1-0.5 s)^{4}\right]
$$

where $s$ is a geometrical parameter and for circular cross section is equal to $4 \delta / D$ and $m$ is the modulus ratio $\left(m=E_{s} / E_{c}\right.$ ). Here $m>1$ and $m<1$ correspond to a surface layer stiffer and softer than the core, respectively and $m=1$ indicates that the surface layer has the same properties as the core. In this study, $m$ was equal to 7 and 8 for cotton- and wood- CNCs, respectively (Figure 6.4). This strengthens the assumption of higher elastic 
modulus on the surface of CNCs and consequently existence of size scale effect in these materials.

Another reason for $\mathrm{CNCs}$ to have higher elastic moduli at smaller diameters can be related to the state of structural defects and size-dependent crystallinity in the material. It can be assumed that the smaller CNCs have a higher degree of crystallinity and therefore fewer molecular defects. The size and crystallinity variations, on the other hand, depend on the CNC preparation process (acid hydrolysis) (Bech-Candanedo et al. 2005). It has been shown that cellulose micro-fibers consist of a crystalline core, amorphous domains, and a paracrystalline surface (Figure 6.5a) (Ioelovich et al. 2010). The amorphous and defective regions in these fibers, which have faster kinematics (Bech-Candanedo et al. 2005), are mostly destroyed during strong acid hydrolysis (Figure 6.5a) (Battista 1975; Fleming et al. 2000; Habibi et al. 2008). During hydrolysis reactions, acids attack firstly the polycrystalline and then the crystalline core of cellulose (Figure 6.5b) (Habibi et al. 2010). Thus, longer reactions result in CNCs with higher crystallinity. This means that, when comparing $\mathrm{CNCs}$ with different sizes from a similar source, there is more crystallinity and fewer defects in smaller CNCs and this can explain the higher elastic moduli in smaller size CNCs.

The size-scale effect has been previously reported for polymeric nanomaterials using both experimental and theoretical approaches (Tan and Lim 2004; Shin et al. 2006; Curgul et al. 2007; Sun et al. 2008). Using AFM, the size scale has been related to the shearing of fibrils within nanofibers in poly L-lactic acid nanofibers (Tan and Lim 2004). Similarly, a three-point bending test in AFM has shown this effect in electro active polymer (EAP) nanofibers and was correlated it to the orientation of polymer molecules during electrospinning (Tan and Lim 2004). Surface elasticity and surface stresses have been used to explain this phenomenon as well. For instance, Curgul et al. (2007), using molecular dynamics (MD) simulations, showed that significant perturbed molecular confirmations occurred at the surface of polyethylene compared to its core. Sun et al. (2008) used a strain gradient theory to predict the size-scale effect in polycaprolactone nanofibers and polypyrrole nanotubes.

The size-scale effect in CNCs can have a substantial role in the engineering of the mechanical properties of functional CNC-based biomaterials. For instance, it has been observed that the osteoblast cells behavior can be regulated by the mechanical properties of the bone scaffolds (Khatiwala et al. 2006). It appears that the control over the size distribution of CNCs can be used to design more effective biomedical implants or tissue replacements. 


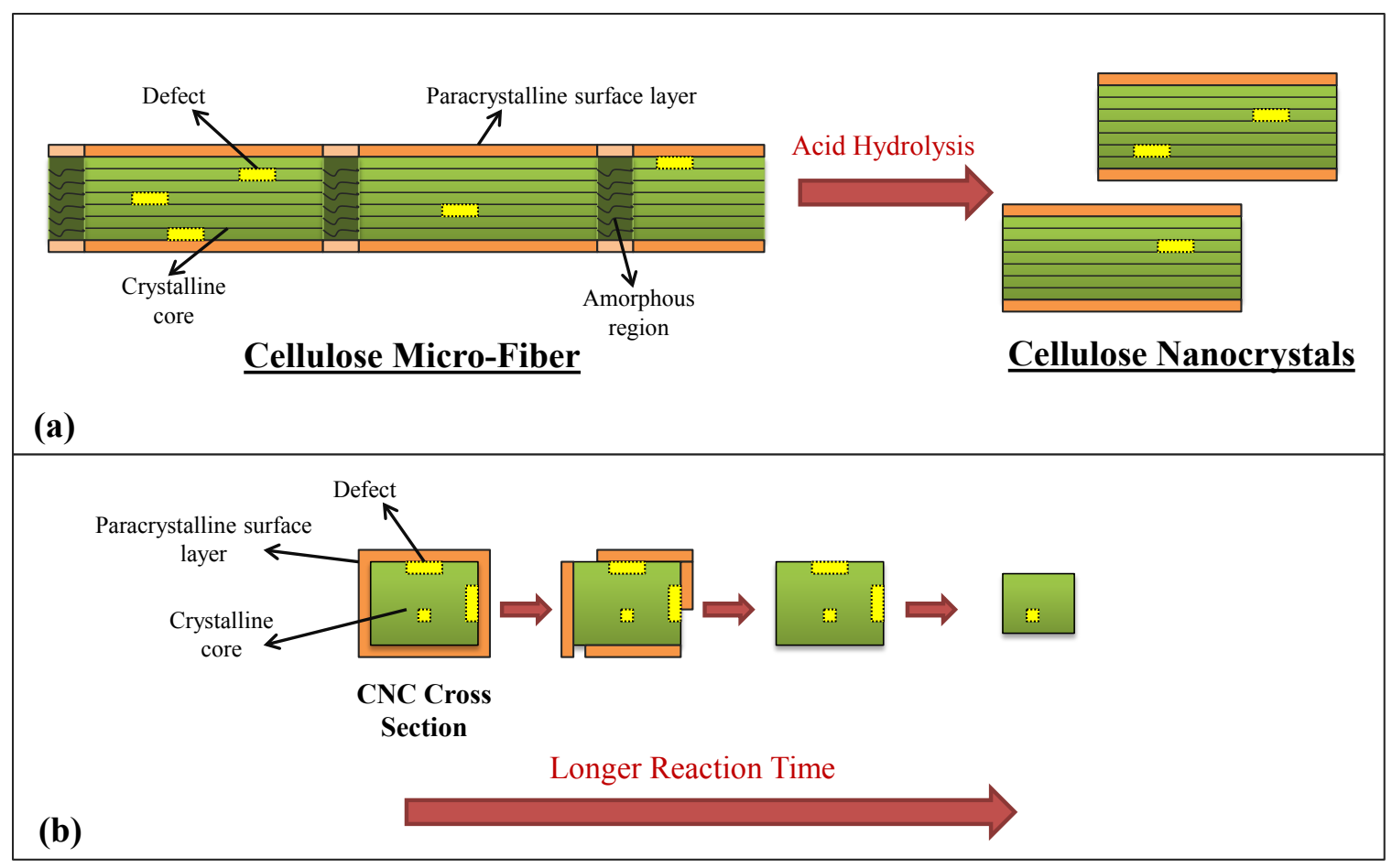

Figure 6.5 (a) Schematic of crystal structure of cellulose along the fiber. Light and dark green correspond to crystalline and amorphous areas in cellulose and orange represents the polycrystalline surface layer. Structural defects are shown by small yellow rectangles. During acid hydrolysis, amorphous regions are removed and cellulose nanocrystals are extracted. (b) Change in cross section of a CNC as the hydrolysis exposure increases. At longer reaction time, acid protons attack the polycrystalline surface and crystalline core of $\mathrm{CNC}$ and resultant crystals will have smaller diameter and fewer defects in their structure.

\subsection{Conclusions}

Acid hydrolysis was used to prepare cellulose nanocrystals (CNCs) from two different sources, namely wood and cotton. It was shown that wood-CNCs had higher length and smaller diameter compared to cotton-CNCs. Nanoindentation was combined with analytical contact mechanics modeling (Hertz model) and finite element analysis (FEA) to estimate the transverse elastic moduli $\left(E_{t}\right)$ of CNCs. It was also observed that the FEA modeling calculated the $E_{t}$ values more closely to the theoretically predictions. The $E_{t}$ for wood- and cotton- CNCs were estimated to be $24.8 \pm 7.0$ and $17.7 \pm 5.0 \mathrm{GPa}$, respectively. The difference in $E_{t}$ for $\mathrm{CNCs}$ from different sources was correlated to the difference between their degrees of crystallinity and diameters. More importantly, it was observed that $E_{t}$ increased when $\mathrm{CNC}$ diameter decreased. Such behavior was explained by the increase of crystallinity ratio and reduction in structural defects. 


\subsection{Acknowledgments}

The authors acknowledge the National Science Foundation (NSF) for the grant No. 0820884 from Division of Materials Research (DMR), and the grant No. 11000806/1100572 from DMR and CMMI divisions. 


\section{Effect of Surface chemistry on Mechanics of Cellulose Nanocrystals}

\subsection{Introduction}

The chemical properties of CNCs on the surface dictate their dispersion properties, the composite fabrication processes, the adhesion between the two phases, and the ultimate composite properties (Moon et al. 2011). Several reviews exist on the functionality and surface modification of cellulose based materials (Hubbe et al. 2008; Moon et al. 2011). In general the $\mathrm{CNC}$ surface modifications can be divided to: (i) reactions to form ionic groups at the cellulosic surfaces, (ii) creation of hydrophobic surfaces and (iii) surface modification by adsorption. Here two of the most commonly used techniques in the first group, where the native surface chemistry of CNCs are modified as a result of extraction methods or similar procedures to treat the surface of CNCs, will be discussed:

Sulfonation: Sulforic acid hydrolysis of cellulose is one of the most common techniques for extraction of CNCs. As shown in Figure 7.1, during this procedure, small amounts of sulfate ester groups are introduced on the crystal (Iwamoto et al. 2009), which results in partial sulfonation of cellulosic surfaces (Beck-Candanedo et al. 2005). These crystals will be called sulfated CNCs ( $\mathrm{S}-\mathrm{CNCs}$ ). This method provides for highly charged surfaces, that make dispersion of CNCs more stabilized (Moon et al. 2011).

Carboxylation: Using treatments that form carboxylic acid on $\mathrm{CNC}$ surfaces, results in more hydrophilic CNCs that disperse better in water (Wagberg et al. 2008). One very effective method for oxidation of CNC surfaces to create carboxyl groups, involves TEMPO-oxidiation (Saito et al. 2005; Iwamoto et al. 2009). During this procedure, C6 hydroxyl group on $\mathrm{CNC}$ changes to carboxylate groups (Figure 7.1). These crystals will be called carboxylated CNCs (C-CNCs). Existence of repulsive forces between the carboxylate groups on the surface makes dispersion of CNCs in water achievable by simple mechanical stirring or sonication.

Although, it has been shown that during these procedures only the surface properties of the crystals change and the internal structure remains intact (Saito et al. 2005), there is no comparison on the properties of CNCs from one source that have been treated differently. In this chapter, CNCs were prepared from cotton in two batches using sulfonation and carboxylation. Then the mechanical and structural properties of these crystals were characterized using atomic force microscopy (AFM) to see weather different surface treatments ulter the properties of the CNCs. 


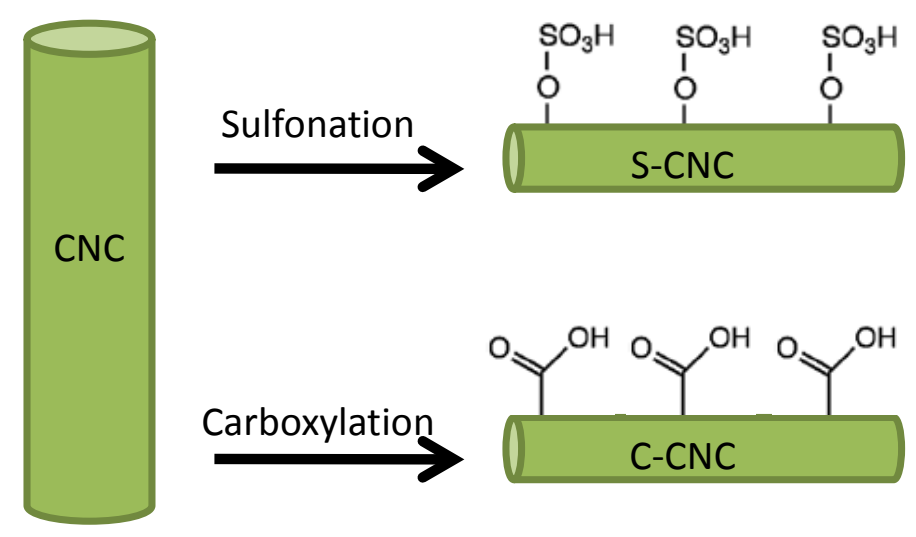

Figure 7.1 Two common syntheses of CNCs that result in different surface properties: suldonation brings sulfate esters (top) and carboxylation results in carboxylic groups (bottom) on the CNC surface.

\subsection{Experimental Procedures}

\subsubsection{Materials}

CNCs were prepared by acid hydrolysis of cellulose as described by Beck-Candanedo et al. (2005). Filter paper (Whatman \#1, Clifton, NJ) was used as the source of cottonCNCs. For this purpose cellulose source in powder was stirred with $65 \% \mathrm{H}_{2} \mathrm{SO}_{4}(v / v)$ for $50 \mathrm{~min}$, at $45{ }^{\circ} \mathrm{C}$. The cellulose-to-acid ratio was $1: 10 \mathrm{~g} / \mathrm{ml}$. After the mixture was centrifuged five times, it was subjected to ultrasonication (Branson Sonifier, Danbury, $\mathrm{CT}$ ) for $15 \mathrm{~min}$, in order to break agglomerations and evenly disperse CNCs in water. To remove remaining salts, ultra filtration was done until the conductivity was $<10 \mu S / c$, (Ultrasette tangential flow UF device, pore size $=50 \mathrm{~nm}$, Pall Corp., Ann Harbor, MI). Finally, an aqueous suspension of $1 \% \mathrm{CNC}$ was obtained by concentrating the dispersion of CNCs in a Rotavaporizer R110 (Buchi, Flawil, Switzerland). These will be called Sulfated (S)-CNCs.

After S-CNCs were prepared by sulfuric acid hydrolysis, a dispersion of $200 \mathrm{~mL}$ of $1 \% \mathrm{CNCs}$ was mixed with $2 \mathrm{~g}$ of $\mathrm{NaBr}$ and $0.2 \mathrm{~g}$ of TEMPO. Then the oxidation reaction was started with the addition of $10 \mathrm{~mL}$ of $\mathrm{NaClO}$ solution and $10 \mathrm{~mL} \mathrm{NaClO}$ solution was repeatedly added after 2 and $4 h$. Overall the reaction took $15 h$. By the addition of $1 N$ $\mathrm{NaOH}$ as necessary $\mathrm{pH}$ was maintained at 10 . Next $30 \mathrm{~mL}$ of ethanol was added to terminate the oxidation reaction. In order to remove unreacted reagents, the suspension was ultrafiltered thrice (Ultrasette tangential flow UF device, pore size $=50 \mathrm{~nm}$, Pall Corp., Ann Arbor, MI). Finally, a calculated amount of $1 \mathrm{M} \mathrm{HCl}$ was added to convert the carboxylates to free acid and the solution was again ultrafiltered until a permeate conductivity of $<5 \mathrm{~S} / \mathrm{cm}$ was obtained. These will be called Carboxylated C-CNCs.

The samples used for AFM imaging were prepared by placing a few drops of CNC dilute solution onto a $1.5 \times 1.5 \mathrm{~cm}^{2}$ of freshly cleaved mica. In order to get a better dispersion of crystals on the mica surface, samples were rinsed with de-ionized water and 
blown dry before the droplets were dry. After rinsing and blow-drying, the CNCs remaining on the mica surface were strongly adhered to the mica.

\subsubsection{Atomic Force Microscopy}

Similar approach to Chaptr 6 was taken here for imaging and characterization of the $\mathrm{CNC}$ mechanical properties. For experimental details and calculations please see sections 6.2.2 and 6.2.3.

\subsubsection{X-ray Diffraction (XRD)}

In order to measure the crystallinity percentage of the samples, a Scintag, Inc. XDS$200 \mathrm{Theta} /$ Theta powder X-ray diffractometer (XRD) with a copper tube was used. CNCs that were prepared as described above were freeze dried. Then the samples were scanned with the rate of $1 \mathrm{Deg} / \mathrm{min}$ from $2 \theta=5-100^{\circ}$ in steps of $0.02^{\circ}$. The crystallinity degree was taken as the ratio of the crystalline diffracted area to the total area by determination of the crystalline and amorphous peaks (Eichhorn and Young 2001).

\subsection{Results and Discussions}

Figure 7.2 shows the transverse elastic moduli of carboxylated and sulfated CNCs versus their diameter. The elastic moduli reported here were obtained by fitting experimental curves with finite element analysis results. In average, S-CNSs and CCNCs were $5.9 \pm 1$ and $3.6 \pm 1.2 \mathrm{~nm}$ in diameter and had $17.7 \pm 5.0$ and $15.1 \pm 4.3 \mathrm{GPa}$ transverse elastic moduli, respectively.

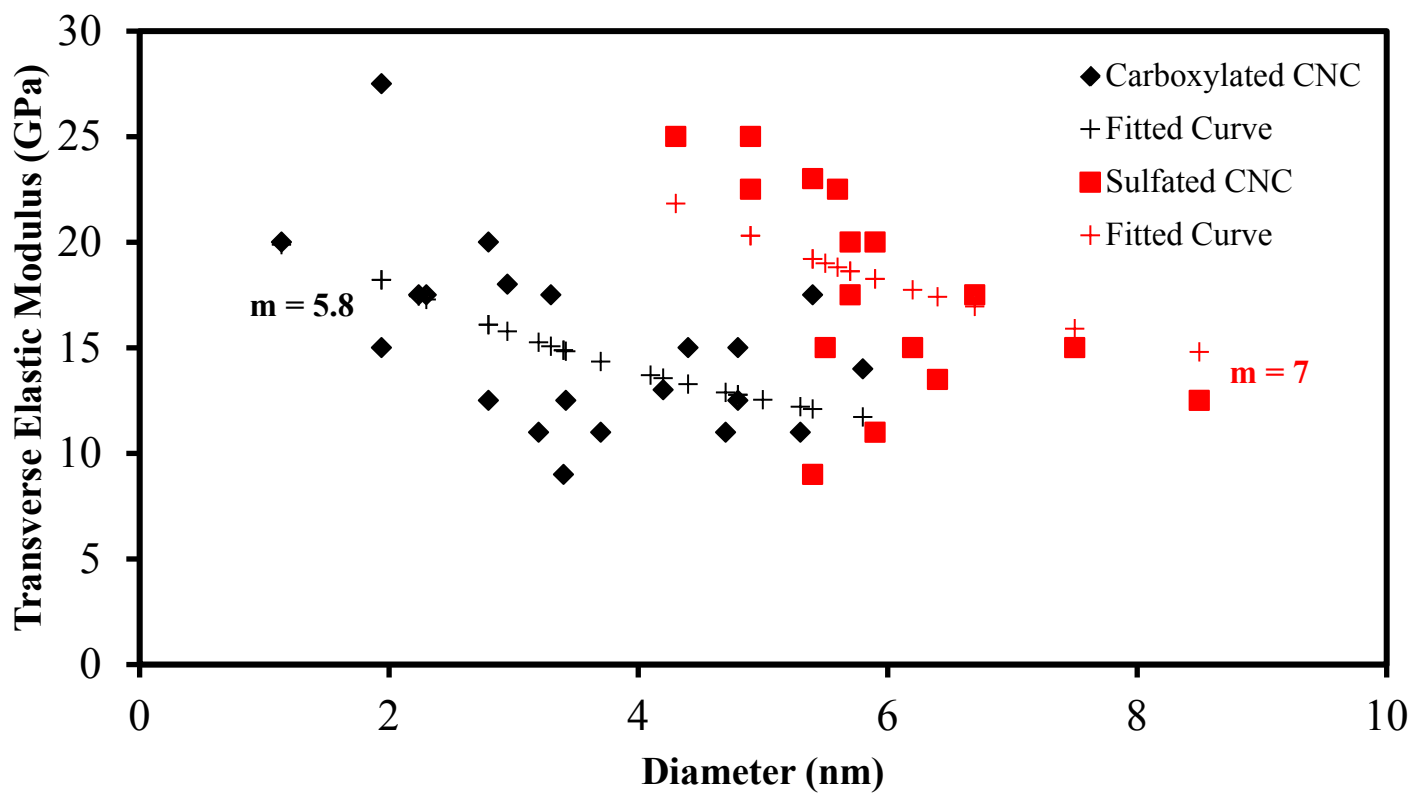

Figure 7.2 Change in $E_{t}$ (Calculated using FEA) with the CNC diameter. Diamonds and squares are the experimental data, and the crosses show the fitted curves, for carboxylated- and sulfated- CNCs. The fitted curves clearly show that as the CNC diameter decreases, $E_{t}$ increases. $m$ resulted from fitting is shown next to the curves. 
For both samples, as the CNC diameter decreased, the transverse elastic modulus increased (size-scale effect). Similar to calculations in Chapter 6, these curves were fitted with theoretical core shell model (Equation 6.7) that resulted in $m=7$ and $m=5.8$, for S$\mathrm{CNCs}$ and C-CNCs, respectively. Again, the fact that $m>1$ strengthens the assumption of higher elastic modulus on the surface of CNC. For further discussion on the size-scale effect in cellulose nanocrystals please see Chapter 6.

X-ray diffraction patterns obtained on S-CNCs and C-CNCs are presented in Figure 7.3. Cotton-CNCs show four different peaks at $2 \theta$ equal to $14.8,16.4,22.5$ and 34.4 that are ascribed to the crystallographic panes of 101, 101, 002, and 040 in cellulose I (Cao and Tan 2004; Wong et al. 2009). Compared to S-CNC, C-CNC had larger crystalline peaks. The crystallinity ratios of sulfated and carboxylated CNCs were found to be $66 \%$ and $70.1 \%$, respectively. These values are close to other XRD data previously reported on cellulose I (Bodenson et al. 2006; Li et al. 2009).

As discussed earlier, it is expected that cellulose crystals with smaller diameter have less defects in their crystalline structure, and as a result higher crystallinity ratio. Similar behavior is being observed here. Compared to S-CNCs (5.9 $\pm 1 \mathrm{~nm}$ in diameter), C-CNCs have smaller diameter $(3.6 \pm 1.2 \mathrm{~nm})$ and XRD showed that the crystallinity ratio in these samples were higher as well. C-CNCs were prepared from S-CNCs via further chemical treatments (see Section 7.2.1). It is expected that these chemical reactions, result in degradation of remaining amourphous regions in S-CNC, thus increase in crystallinity ratio.

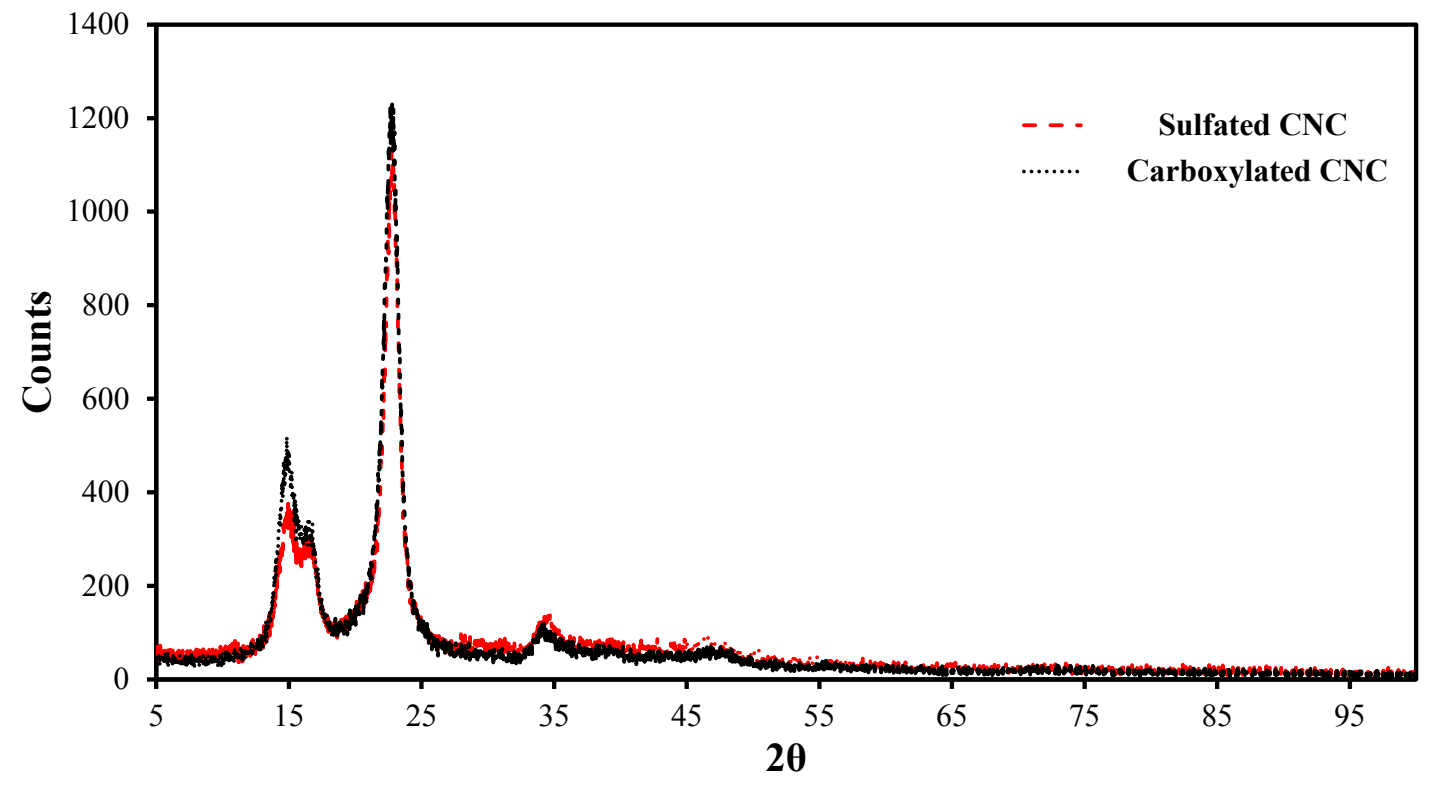

Figure 7.3 Powder diffraction patterns for sulfated (dashed red line) and carboxylated (dotted black line) CNCs. Compared to S-CNC, C-CNC had larger crystalline peaks. 


\subsection{Conclusions}

Carboxylated and sulfated CNCs were prepared from cotton and their mechanical and structural properties were compared. Carboxylation results in CNCs with smaller diameter and as a result, less defects and higher crystallinity ratio. Sulfated CNCs had higher transverse elastic moduli.

\subsection{Acknowledgments}

The authors thank Mr. E. Laitila at Materials Science Department who performed the XRD tests. 


\section{Conclusions}

In order for cellulose crystals to substitute petroleum based reinforcements used in industrial scales, the mechanical properties of cellulose crystals and their effect on the mechanics of the polymer composite need to be well-characterized. For this reason:

- SEM imaging and X-ray ultra microtomography (XuM) were used to study the relationship between the dispersion of cellulose microcrystals and tensile properties of PCL composites. 3D reconstructed volumes provided supplementary information and made explanation of mechanical behavior of the samples possible. The size, shape and distribution of CMC clusters, had high effects on the void formation and crack propagation rates in the materials and weak bonding between $\mathrm{CMC}$ and PCL resulted in reduction of UTS in all composite samples. On the other hand, $10 \mathrm{wt} \% \mathrm{CMC}$ improved the flow of polymer molecules under stress and improved the ductility and toughness of PCL.This implies that, in order to better understand and characterize the properties of composite systems the 2D information obtained from SEM and TEM imaging may not be sufficient and other approaches such as XuM need to be taken.

- Nanomechanical properties of cellulose nanocrystal composites were characterized at the surface (nanoindentation in AFM). These were compared with their macro tensile properties. Except for the composite with $15 w t . \% \mathrm{CNC}$, the elastic moduli from nanoindentation followed the same trend as those obtained from tensile tests. Tensile tests showed that addition of CNC up to $20 \mathrm{wt}$. \% improved the elastic modulus of PVA, when PAA was present in the composition. Also, in the absence of PAA, agglomeration of CNCs resulted in nanocomposite membrane with lowest elastic modulus. On the other hand, nanoindentation tests showed that addition of only $15 \mathrm{wt} \% \mathrm{CNC}$ improved the elastic modulus of the composite similar to the case where $10 \mathrm{wt} . \% \mathrm{CNC}$ and $10 \mathrm{wt} . \%$ PAA was added to PVA.

- Quantitative nanomechanical properties of the interphase (the zone between CNC surface and polymer matrix) were characterized in cellulose nanocrystal composites using peak force tapping mode in AFM. The properties at the interphase were correlated with the structural properties of the polymer matrix. It was shown that the thickness of the interphase was dependent on the diameter of cellulose nanocrystals and increased with increase in diameter of CNC. This was correlated to higher surface area and as a result more penetration of $\mathrm{CNC}$ influence in the polymer matrix for $\mathrm{CNCs}$ with larger diameter.

- Lastly, cellulose nanocrystals from wood and cotton were prepared and compared with respect to their structure and mechanics. Nanoindentation in AFM was used to measure the transverse elastic modulus of these crystals and two different approaches (contact mechanics modeling and finite element analysis) were used to analyze the data. FEA modeling calculated the $E_{t}$ values more closely to the theoretical predictions. It was shown that compared to cotton-CNCs, in average cellulose nanocrystals from wood had smaller diameter and higher elastic modulus. This was correlated to the difference between the crystallinity of these two materials. Additionally, we observed that for both cotton and wood-CNCs $E_{t}$ increased when $\mathrm{CNC}$ diameter decreased. This was also 
correlated to decrease of imperfections and increase of the crystallinity in CNCs with smaller diameter. 


\section{Future Works}

In current $\mathrm{PhD}$ project mechanical properties of cellulose nanocrystals and their polymer composites were studied. This area of research has great potentials and there are numerous directions that can be taken in the future in continuation of the current work. In this chapter a few of these ideas are presented.

\subsection{D Distribution of CNCs in Polymer Composites}

In the $3^{\text {rd }}$ chapter of this dissertation, direct correlation was made between $\mathrm{CMC}$ distribution and mechanical properties of PCL composites. Similar to CMCs, dispersion of CNCs in different polymer composites has great influence on mechanical properties of final material. On the other hand the size of these crystals and limited spatial resolution of XuM, specifically in 3D (not less than $200 \mathrm{~nm}$ ), makes it very difficult to characterize the distribution of CNCs using this method. In order to perform similar studies on cellulose nanocomposites, we propose application of a new method called serial block face imaging (Denk and Horstmann, 2004), which provides reconstructed volumes with resolution of a scanning electron microscope. This technique has been used for various biological materials (Briggman et al. 2011) and can be performed in environmental mode SEM to avoid charging of the surface. This techniques uses back scattered electron imaging in SEM. In order to provide sufficient contrast between CNCs and the polymer matrix, it is recommended to use cellulose nanocrystals that have been functionalized with gold nanoparticles (Mahmoud et al. 2009).

\subsection{Interphase Characterization}

In Chapter 5, nanomechanical properties of cotton CNC-PVA composites at the interphase were investigated and it was shown that the interphase thickness is a function of CNC diameter. Following this study:

1- The effect of polymer type (hydrophilic or hydrophobic (Heux et al. 2000; Grunert and Winter 2002; Gopalan Nair et al. 2003), CNC source (wood and agricultural byproducts (Beck-Candanedo et al. 2005), animal cellulose (Sturcova et al. 2005) and bacteria (Grunert and Winter 2002)), CNC surface properties (changed by physical methods (Heux et al. 2000) and/or chemical modifications (Gong et al. 2011)) and CNC volume ratio should be studied. In order to better understand the effect of each of these parameters on the interphase thickness as well as the ultimate mechanical properties, a series of well designed experiments should be performed by keeping all these parameters constant and changing only one at each set of experiments.

For instance, a set of experiments can be done to characterize the effect of CNC type on the interphase properties. For this purpose, a polymer matrix should be reinforced with similar contents of CNCs from different sources. Then the interphase should be characterized using the same methods used in Chapter 5. Does the interphase thickness change with diameter in composites reinforced with other kinds of CNC than cotton? What happens if CNCs from different sources, but with similar diameters are used? Do 
the interphase mechanical properties depend on the crystallinity ratio of the CNCs? These are some of several questions that can be answered.

2- Additionally a series of mechanical tests in macro scale is needed to make a correlation between the interphase thickness and the macro scale mechanical properties. The interphase thickness is dependent on the CNC size, thus in order for these experiments to be meaningful CNCs with more uniform size distribution are needed. It is recommended that before addition of CNCs to the polymer matrix, ultracentrifugation be used as explained in Chapter 2, to separate CNCs with different diameters.

3- A few studies exist on theoretical modeling of the interphase in nanocomposite systems (Brown et al. 2008; Jancar et al. 2010; Li et al. 2011). These models fail to predict the effect of the interphase thick on nanocpmposite properties. On the other hand, specifically in CNC composites, we showed that the interphase thickness is not constant. Thus, new theoretical models are needed to more accurately predict nanocomposite behaviors with consideration of the effect of the interphase thickness.

\subsection{CNC and CNC Composites for Biomedical Applications}

One of the major motivations in material science is to find or design biocompatible materials that can be used in biomedical application. Cellulose is known to be biocompatible and biodegradable (Miyamoto et al. 1989) and it has mechanical properties that can be tuned to be similar to soft and hard tissue. Thus, it can be used either by itself (pure CNCs) or as a reinforcing agent in various areas such as drug delivery, tissue engineering and medical implants.

1- Use of mechanically strong scaffolds in tissue engineering is essential in order to support cell grows and define the shape of the new growing tissue. An important factor in this method is to secure sufficient adhesion of cells to the scaffold. Bacterial cellulose has been shown to have great potentials in cartilage and bone tissue engineering (Svensson et al. 2005; Wan et al. 2006). Surprisingly, no reports exist on application of cellulose from other resources for these purposes. The compatibility and adhesion of osteoblast cells to cellulose nanocrystals from different sources should be investigated. This can be performed by preparation of CNCs from different sources, placing of a layer of CNC films on a mica substrate and growing cells on the films. AFM can then be used in tapping mode to image different substrates and to find if the cells adhere differently to CNCs from different sources.

2- Bionanocomposites combine biology, material science, and nanotechnology and form an interesting interdisciplinary area. There are several applications and different functional requirements for these materials in biomedicine. One of the areas that has been more investigated during past few years is production of bionanocomposites based on biomimetic approaches. Amongst various systems, hydroxyapetite (HAP)-polymer nanocomposites have been applied to bone repair and implantation (Hule and Pochan 2007). In particular, bacterial cellulose-HAP nanocpmposites have been greatly investigated (Wan et al. 2007; Hofman et al. 2008). Using the information obtained in 1, other $\mathrm{CNC}$ sources than bacteria can be selected. It would be interesting to see if the 
mechanical properties and as a result the applications of these composites can be tuned by changing the $\mathrm{CNC}$ source. 


\section{Reference List}

Abdelmouleh M, Boufi S, ben Salah A, Belgacem MN, Gandini A. 2002. Interaction of silane coupling agents with cellulose. Langmuir. 18: 3203-3208.

Aabloo A, French AD. 1994. Preliminary potential energy calculations of cellulose I $\alpha$ crystal structure. Macromolecular Theory and Simulations. 3:185.

Abdul Khalil HPS, Bhat AH, Yusra I. 2011. Green composites from sustainable cellulose nanofibers: A review. Carbohydrate Polymers. doi:10.1016/j.carbpol.2011.08.078.

Alemdar A, Sain M Biocomposites. 2007. From wheat straw nanofibers: Morphology, thermal and mechanical properties. Composites Science and Technology. 68:557-565.

Angles MN, Dufresne A. 2000. Plasticized starch/tunicin whiskers nanocomposites. 1. Structural analysis. Macromolecules. 33:8344-8353.

Angles MN, Dufrense A. 2001. Plasticized starch/tunicin whisker nanocomposite materials 2: Mechanical behavior. Macromolecules. 34:2921-2931.

Araki J, Wada M, Kuga S. 2001. Steric stabilization of a cellulose microcrystal suspension by poly(ethylene glycol) grafting. Langmuir. 17: 21-27.

Araki J, Wada M, Kuga S, Okano T. 1998. Flow properties ofmicrocrystalline cellulose suspension prepared by acid treatment of native cellulose. Colloids and Surfuces. 142: 75-82.

Arndt K, Richter A, Ludwig S, Zimmermann J, Kressker J, Kuckling D, Alder H. 1999. Poly(vinyl alcohol)/ poly(acrylic acid) hydrogels: FT-IR spectroscopic characterization of crosslinking reaction and work at transition point. Acta Polymers. 50:383-390.

Asif SAS, Wahl KJ, Colton RJ. 2000. The influence of oxide and adsorbates on the nanomechanical response of silicon surfaces. Journal of Materials Research. 15:546-553.

Atalla RH, VanderHart D. 1984. Native Cellulose: a composite of two distinct crystalline forms. Science. 223:283.

Auad ML, Contos V S, Nutt S, Aeanguren MI, Marcovich NE. 2008. Characterization of nanocellulose reinforced shape memory polyurethanes. Polymer International. 57:651659.

Azizi Samir M, Alloin F, Dufresne A. 2005. Review of recent research into cellulosic whiskers, their properties and their applications in nanocomposite field. Biomacromolecules. 6:612-626. 
Azizi Samir M, Alloin F, Sanchez J, Dufresne A. 2004-a. Cellulose nanocrystals reinforced poly(oxyethylene). Polymer. 45:4149-4157.

Azizi Samir M, Alloin F, Sanchez J, Kissi NE, Dufresne A. 2004-b. Preparation of cellulose whiskers reinforced nanocomposites from an organic medium suspension. Macromolecules. 37:1386-1393.

Baker AA, Helbert W, Sugiyama J, Miles MJ. 2000. New insight into cellulose structure by atomic force microscopy shows the $\mathrm{I}_{\alpha}$ crystals phase at near-atomic resolution. Biophysical Journal. 79:1139-1145.

Batista OA. 1975. Microcrystal Polymer Science. New York, New York: McGrawHill Book Company.

Battista OA, Coppick S, Howsmon JA, Morehead FF, Sisson WA. 1956. Level-off degree of polymerization. Industrial and Engineering Chemistry Research. 48:333-335.

Beake BD, Laggett GJ. 2002. Nanoindentation and Nano-scratch testing of Uniaxially and Biaxially Drawn Poly(ethylene terephthalate) Film. Polymer. 43: 319-327.

Beck-Candanedo S, Roman M, Gray D G. 2005. Effect of reaction conditions on the properties and behavior of wood cellulose nanocrystal suspensions. Biomacromolecules. 6:1048-1054.

Ben Mubarak A, Kaddami H, Magnin A, Belgacem MN, Dufresne A, Boufi S. 2011. Preparation of nanocomposite dispersions based on cellulose whiskers and acrylic copolymer by miniemulsion polymerization: effect of silane content. Polymer Engineering and Science. 51: 62-70.

Bischel MS, VanLandingham MR, Eduljee RF, Gillespie JW, Schultz JM. 2000. On the use of nanoscale indentation with the AFM in the identification of phases in blends of linear low density polyethylene and high density polyethylene. Journal of Materials Science. 35: 221-228.

Bodeson D, Mathew A, Oksman K. 2006. Optimization of the isolation of nanocrystals from microcrystalline cellulose by acid hydrolysis. Cellulose. 13:171-180.

Bodenson D, Oksman K. 2007. Dispersion and characterization of surfactant modified cellulose whiskers nanocomposites. Composite Interfaces. 14:617-630.

Bolshakov A, Pharr GM. 1998. Influences of pile-up on the measurement of mechanical properties by load and depth sensing indentation techniques. Journal of Material Research. 13:1049-1058.

Bonse U, Bush F. 1996. X-ray computed microtomography using synchrotron radiation. Progress in Biophysics and Molecular Biology. 65:133-169. 
Bourke S, Al-Khalili M, Briggs T, Michniak B, Kohn J, Poole-Warren L. 2003. A photo-crosslinked poly(vinyl alcohol) hydrogel growth factor release vehicle for wound healing applications. AAPS PharmSci. 5: Article 33.

Briggman KL, Helmstaedter M, Denk W. 2011. Wiring specificity in the directionselectivity circuit of retina. Nature. 471: 183-190.

Brown D, Marcadon V, Mele P, Alberola ND. 2008. Effect of filler particle size on the properties of model nanocomposites. Macromolecules. 41:1499-1511.

Brown EE, Laborie MG, 2007. Bioengineering bacterial cellulose/poly (ethylene oxide) nanocomposites. Biomacromolecules. 8:3074-3081.

Cadek M, Hedicke K, Blau WJ. 2002. Morphological and mechanical properties of carbon nanotube reinforced semicrystalline and amorphous polymer composites. Applied Physics Letters. 81: 5123-5125.

Cao Y, Tan H. 2004. Structural characterization of cellulose with enzymatic treatment. Journal of Molecular Structure. 705: 189-193.

Cao X, Dong H, Li CM. 2007. New nanocomposite materials reinforced with flax cellulose nanocrystals in waterborne polyurethane. Biomacromolecules. 8:899-904.

Cappella B, Dietler G. 1999. Force-distance curves by atomic force microscopy. Surface Science Reports. 34:1-104.

Cappella B, Kaliappan SK, Sturum H. 2005. Using AFM force-distance curves to study the glass-to-rubber transition of amorpjous polymers and their elastic-plastic properties as a function of temperature. Macromolecules. 38: 1874-1881.

Carotenuto GC, Her YS, Matijevic E. 1996. Preparation and characterization of nanocomposite thin films for optical devices. Industrial and Engineering Chemistry Research. 35:2929-2932.

Cheng Q, Wang S. 2008. A method for testing the elastic modulus of single cellulose fibrils via atomic force microscopy. Composites. 39:1838-1843.

Cheng Q, Wang S, Rials TG, Lee S. 2007. Physical and mechanical properties of polyvinyl alcohol and polypropylene composite materials reinforced with fibril aggregates isolated from regenerated cellulose fibers. Cellulose. 14: 592-602.

Cherian BM, Leao AL, de Souza SF, Costa KMM, de Olyveira GM, Kottaisamy M, Nagarajan ER, Thomas S. 2011. Cellulose nanocomposites with nanofibers isolated from pineapple leaf fibers for medical applications. Carbohydrate Polymers. 86: 1790-1798.

Chizhik SA, Huang Z, Gorbunov VV, Myshkin NK, Tsukruk VV. 1998. Micromechanical properties of elastic polymeric materials as probed by scanning force microscopy. Langmuir. 14:2606-2609. 
Choi Y, Simonsen J. 2006. Cellulose nanocrystal-filled carboxymethyle cellulose nanocomposites. Journal of Nanoscience and Nanotechnology. 6:633-639.

Cosslett VE, Nixon WC. 1951. Improved Resolution with the X-Ray Projection Microscope. Nature. 168:24-25.

Cosslett VE, Nixon WC. 1960. X-ray Microscopy. London: Cambridge university press.

Curgul S, VanVliet KJ, Rutledge GC. 2007. Molecular dynamics simulation of size dependent structural and thermal properties of polymer nanofibers. Macromolecules. 40:8483-8489.

Dahneke BE. 1983. Measurement of suspended particles by quasi-elastic light scattering. New York: Wiley.

Denk W, Horstmann H. 2004. Serial block-face scanning electron microscopy to reconstruct three dimensional tissue nanostructure. PLoS Biolology. 2:e329.

De Souza Lima MM and Borsali R. 2002. Static and dynamic light scattering from polyelectrolyte microcrystal cellulose. Langmuir. 18:992-996.

Derjaguin BV, Muller VM, Toropov Y. 1975. Effect of Contact Deformations on the Adhesion of Particles. Journal of Colloidal Interface Science. 53:314-326.

Dibenedetto AT, Scola DA. 1978. Characterization of S-glass-polymer interfaces using ion scattering spectroscopy and ion mass spectroscopy. Journal of Colloid and Interface Science. 64:480-500.

Dierick M, Vlassenbroeck J, Masschaele B, Cnudde V, van Hoorebeke L, Hillenbach A. 2005. High-speed neutron tomography of dynamic processes. Nuclear Instrumemnts and Methods in Physics Research A. 542:296-301.

Dimitriadis EK, Horkay F, Maresca J, Kachar B, Chadwick RS. 2002. Determination of elastic moduli of thin layers of soft material using the atomic force microscope. Biophysics Journal. 82:2798-2810.

Domke J, Radmacher M. 1998. Measuring the elastic properties of thin films with the atomic force microscope. Langmuir. 14:3320-3325.

Downing TD, Kumar R, Cross WM, Kjerengtroen L, Kellar JJ. 2000. Determining the interphase thickness and properties in polymer matrix composites using phase imaging atomic force microscopy and nanoindentation. Journal of Adhesion Science and Technology. 14:1801-1812. 32.

Drzal LT. 1986. The interphase in epoxy composites. Advance Polymer Science. 75:1- 
Droste DH, Dibenedetto AT, Stejskal EO. 1971. Multiple phases in filled polymers detected by nuclear spin relaxation studies. Journal of Polymer Science. 9:187-189.

Du B, Liu J, Zhang Q, He T. 2001. Experimental measurement of polyethylene chain modulus by scanning force microscopy. Polymer. 42: 5901-5907.

Dufresne A, Dupeyre D, Vignon MR. 2000. Cellulose microfibrils from potato tuber cells: processing and characterization of starch-cellulose microfibril composites. Journal of Applied Polymer Science. 76:2080-2092.

Eichhorn S J, Dufresne A, Aranguren M, Marcovich NE, Capadona JR, Rowan SJ, Weder C, Thielemans W, Roman M, Renneckar S, Gindl W, Veigel S, Keckes J, Yano H, Abe K, Nogi M, Nakagaito AN, Mangalam A, Simonsen J, Benight AS, Bismarck A, Berglund LA, Peijs T. 2010. Review: current international research into cellulose nanofibres and nanocomposites. Journal of Materials Science. 45:1-33.

Eichhorn SJ, Sirichaisit J, Young RJ. 2001a. Deformation mechanisms in cellulose fibers, paper and wood. Journal of Materials Science. 36:3129-3135.

Eichhorn SJ, Young RJ. 2001. The Young's Modulus of a microcrystalline cellulose. Cellulose. 8:197

Eichhorn SJ, Young RJ, Yeh WY. 2001b. Deformation processes in regenerated cellulose fibers. Textile Research Journal. 71:121-129.

Elazzouzi-Hafraoui S, Nishiyama Y, Pataux J, Heux L, Dubreuil F, Rochas C. 2008. The shape and size distribution on crystalline nanoparticles prepared by acid hydrolysis of native cellulose. Biomacromolecules. 9:57-65.

Favier V, Chanzy H, Cavaille JY. 1995. Polymer nanocomposites reinforced by cellulose whiskers. Macromolecules. 28:6365-6367.

Favier V, Ganova GR, Shrivastava SC, Cavaille JY. 1997. Mechanical percolation in cellulose whisker nanocomposites. Polymer Engineering and Science. 37:1732-1739.

Feldkamp LA, Davis LC, Kress JW. 1984. Practical cone-beam algorithm. Journal of Optical Society of America. 1:612-619.

Feng G, Yoon Y, Lee CJ. 2006. A study of the mechanical properties of nonowires using nanoindentation. Journal of Applied Physics. 99:074304.

Fischer-Cripps AC. 2004. Nanoindentation. New York: Springer Verlag.

Finch C. 1992. Polyvinyl Alcohol Developments. New York: John Wiley \& Sons.

Finkenstadt VL, Millane RP. 1998. Crystal structure of Valonia cellulose I $\beta$. Macromolecules. 31:7776-7783. 
Fleming K, Gray D, Prasannan S, Matthews S. 2000. Cellulose crystallites: A new and robust liquid crystalline medium for the measurement of residual dipolar couplings. Journal of the American Chemical Society. 122:5224-5225.

Gaillard Y, Tromas C, Woirgard. 2006. Quantitative analysis of dislocation pile-ups nucleated during nanoindentation in MgO. Acta Materialia. 54:1409-1417.

Gao SL, Mader E. 2002. Characterization of interphase nanoscale property variations in glass fibre reinforced polypropylene and epoxy resin composites. Composites, Part A. 33:559-576.

Gardner DJ, Oporto JS, Mills R, Azizi Samir A. 2008. Adhesion and Surface Issues in Cellulose and Nanocellulose. Journal of Adhesion Science and Technology. 22: 545-567.

George J, Sreekala MS, Thomas S. 2001. A review on interface modification and characterization of natural fiber reinforced plastic composites. Polymer Engineering and Science. 41:1471-1485.

Giannelis EP. 1996. Polymer-layered silicate nanocomposites. Advanced Materials. 8:29-35.

Glasser WG. 2004. Prospects for future applications of cellulose acetate. Macromolecular Symposia. 208:371-394.

Goffin AL, Raquez JM, Duquesne E, Siqueira G, Habibi Y, Dusfresne A, Dubois Ph. 2011. Poly (caprolactone) based nanocomposites reinforced by surface grafted cellulose nanowhiskers via extrusion processing: morphology, rheology and thermo-mechanical properties. Polymer. 52: 1532-1538.

Goldstein J, Newbury D, Joy D, Lyman C, Echlin P, Lifshin E, Sawyer L, Michael J. 2003. Scanning Electron Microscopy and X-ray Microanalysis. New York, New York: Springer Science + Buisiness Media.

Gong G, Pyo J, Mathew AP, Oksman K. 2011. Tensile behavior, morphology and viscoelastic analysis of cellulose nanofibers-reinforced (CNF) polyvinyl acetate (OVAc). Composites. 42: 1275-1282.

Gopalan Nair K, Dufresne A, Gandini A, Belgacem MN. 2003. Crab shell chitin whiskers reinforced natural rubber nanocomposites 3.Effect of chemical modification of chitin whiskers. Biomacromolecules. 4:1835-1832.

Grunert M, Winter WT. 2002. Nanocomposites of cellulose acetate butyrate reinforced with cellulose nanocrystals. Journal of Polymers and the Environment. 10:27-30.

Guhados G, Wan W, Hutter JL. 2005. Measurement of single bacterial cellulose fibers using atomic force microscopy. Langmuir. 21:6642-6646.

Habibi Y, Dufresne A. 2008. Highly filled bionanocomposites from functionalized polysaccharide nanocrystals. Biomacromolecules. 9:1974-1980. 
Habibi Y, Goffin AL, Schiltz N, Duquesne E, Dubois P, Dufresne A. 2008. Bionanocomposites based on poly(E-caprolactone)-grafted cellulose nanocrystals by ring-opening polymerization. Journal of Material Chemistry. 18:5002-5010.

Habibi Y, Lucia LA, Rojas OJ. 2010. Cellulose Nanocrystals: Chemistry, SelfAssembly, and Applications. Chemical Reviews. 110:3479-3500.

Hajji P, Cavaille JY, Favier V, Gauthier C, Vigier G. 1996. Tensile behavior of nanocomposites from latex and cellulose whiskers. Polymer Composites. 17:612-619.

Hamad W. 2006. On the development and applications of cellulosic nanofibrillar and nanocrystalline materials. The Canadian Journal of Chemical Engineering. 84:513-519.

Harris B. 1999. Engineering composite materials. London: IOM Communications Ltd.

Hashin Z. 1990. Thermoelastic properties of fiber composites with imperfect interface. Mechanics of Materials. 8: 333-348.

Helbert W, Cavaille JY, Dufresne A. 1996. Thermoplasric nanocomposites filled with wheat straw cellulose whiskers. Part I: processing and mechanical behavior. Polymer Composites. 17:604-611.

Hertz HJ. 1881. On the contact of rigid elastic solids and hardness. Journal of Reine Angew Math. 92:156-171.

Heux L, Chauve G, Bonnini C. 2000. Nanoflocculating and chiral-nematic selfordering of cellulose microcrystals suspensions in nonpolar solvents. Langmuir. 16:82108212.

Hodzic A, Stachurski ZH, Kim JK. 2000. Nanoindentation of polymer-glass interfaces Part I. Experimental and mechanical analysis. Polymer. 41:6895-6905.

Hofmann I, Haas D, Eckert A, Ruf H, Firgo H, Muller FA, Greil P. 2008. Mechanical properties of cellulose-apetite composite fibers for biomedical applications. Advances in Applied Ceramics. 107: 293-297.

Hon DSN. 1994. Cellulose: a random walk through its historical path. Cellulose. 1:125 .

Hongmei W, Chen Z, Fang P et al. 2007.Synthesis, characterization and optical properties of hybridized CdS-PVA nanocomposites. Materials Chemistry and Physics. 106:443-446.

Hsieh YC, Yano H, Nogi M, Eichhorn SJ. 2008. An estimation of the Young's modulus of bacterial cellulose filaments. Cellulose. 15:507-513.

Huang M, Liu L, Zhang G, Yuan G, Fang Y. 2006. Preparation of chitosan derivative with polyethylene glycol side chains for porous structure without specific processing technique. International Journal of Biological Macromolecules. 38: 191-196. 
Hubbe MA, Rojas OJ, Lucia LA, Sain M. 2008. Cellulose Nanocomposites: A review. BioResources. 3: 929-980.

Hule RA, Pochan DJ. 2007. Polymer nanocomposites for biomedical applications. MRS Bulletin. 32: 354-358.

Hyder MN, Huang RYM, Chen P. 2006. Journal of Membrane Science. Correlation of physicochemical characteristics with pervaporation performance of poly(vinyl alcohol) membranes. 283:281-290.

Ioelovich M, Leykin A, Fogovsky O. 2010. Study of cellulose paracrystallinity. Bioresources. 5:1393-1407.

Iwamoto S, Kai W, Isogai A, Iwata T. 2009. Elastic modulus of single cellulose microfibrils from tunicate measured by atomic force microscopy. Biomacromolecules. $10: 2571-2576$.

Ishida H, Koenig JL. 1978. Fourier-transform infrared spectroscopic study of silane coupling agent-porous silica interface. Journal of Colloid and Interface Science. 64:555565.

Jancar J, Douglas JF, Starr FW, Kumar SK, Gassagnau P, Lesser AJ, Strenstein SS, Buehler MJ. 2010. Current issuesin research on structure-property relationships in polymer nanocomposites. Polymer. 51:3321-3343.

Jaswon A, Gillis PP, Mark RE. 1968. The elastic constants of crystalline native cellulose. Proceedings of the Royal Society of London. 306:389-412.

Jee A, Lee M. 2010. Comparative analysis on the nanoindentation of polymers using atomic force microscopy. Polymer Testing. 29:95-99.

Jennings B, Parslow K. 1988. K particle size measurement: The equivalent spherical diameter. Proceedings of the Royal Society of London. 419:137-149.

Johnson KL, Kendall K, Roberts AD. 1971. Surface energy and the contact of elastic solids. Proceedings of the Royal Society A. 324: 301-313.

Karapanagiotis I, Evans DF, Gerberich WW. 2002. Dynamics of the Leveling Process of Nanoindentation Induced Defects on Thin Polystyrene Films. Polymer. 43:1343-1348.

Khatiwala CB, Peyton SR, Putnam AJ. 2006. Intrinsic mechanical properties of the extracellular matrix affect the behavior of pre-osteoblastic MC3T3-E1 cells. American Journal of Physiology, Cell Physiology. 290:1640-1650.

Klapperich C, Komvopoulos K, Pruitt L. 2001. Nanomechanical properties of polymers determined from nanoindentation experiments. Transaction of ASME. 123:624631. 
Kojima Y, Usuki A, Kawasumi M, Okada A, Kurauchi T, Kamigaito O. 1993. Onepot synthesis of nylon-6 clay hybrid. Journal of Polymer Science. 31:1755-1758.

Kojimia Y, Usuki A, Okada A. 1997. Synthesis and properties of polyimide-clay hybrid films. Journal of Polymer Science. 35: 2289-2294.

Kong $\mathrm{K}$ and Eichhorn SJ. 2005. The influence of hydrogen bonding on the deformation micromechanics of cellulose fibers. Journal of Macromolecular Science. 44:1123-1136.

Koo CM, Kim MJ, Choi MH, Kim SO, Chung IJ. 2003. Mechanical and rheological properties of the maleated polypropylene-layered silicate nanocomposites with different morphology. Journal of Applied Polymer Science. 88:1526-1535.

Kovalenko VI. 2010. Crystalline Cellulose: Structure and hydrogen bonds. Russian Chemical Reviews. 79:231.

Kroon LMJ, Kroon L, Northolt MG. 1986. Chain modulus and intramolecular hydrogen bonding in native and regenerated cellulose fibers. Polymer Communications. 27:290-292.

Kumeta K, Nagashima I, Matsui S, Mizoguchi K. 2003. Crosslinking reaction of poly(vinyl alcohol) with poly(acrylic acid) by heat treatment: Effect of neutralization of PAA. Journal of Applied Polymer Science. 90:2420-2427

Kvien I, Sugiyama J, Votrubec M, Oksman K. 2007. Characterization of starch based nanocomposites. Journal of Materials Science. 42:8163-8171.

Kvien I, Tanem BS, Oksman K. 2005. Characterization of cellulose whiskers and their nanocomposites by atomic force and electron microscopy. Biomacromolecules. 6:31603165

Lahiji RR, Xu X, Reifenberger R, Roman A, Rudie A, Moon RJ. 2010. Atomic Force Microscopy Characterization of Cellulose Nanocrystals. Langmuir. 26:4480-4488.

Laiarinandrasana L, Morgeneyer TF, Proudhon H, Regrain C. 2010. Damage of semicrystalline polyamide 6 assessed by 3D x-ray tomography: from microstructure evolution to constitutive modeling. Journal of Polymer Science B. 48:1516-1525.

Lapa VLC, Suarez JCM, Visconte LLY, Nunes RCR. 2007. Fracture behavior of nitrile rubber-cellulose II nanocomposites. Journal of Material Science. 42:9934-9939.

Lee S, Wang S, Pharr GM, Xu H. 2007. Evaluation of interphase properties in a cellulose fiber reinforced polypropylene composites by nanoindentation and finite element analysis. Composites. 38:1517-1524 .

Li R, Fei J, Cai Y, Li Y, Feng J, Yao J. 2009.Cellulose whiskers extracted from mulberry: A novel biomass production. Carbohydrate Polymers. 76: 94-99. 
Li Y, Waas AM, Arruda EM. 2011. A closed-form, hierarchical, multi-interphase model for composites-derivation, verification and application to nanocomposites. Journal of the Mechanics and Physics of Solids. 59:43-63.

Lima MMS, Borsali R. Rodlike cellulose microcrystals: structure, properties, and applications. 2004. Macromolecular Rapid Communications. 25: 771-787.

Liu H, Brinson CL. 2008. Reinforcing efficiency of nanoparticles: A simple comparison for polymer nanocomposites. Composites Science and Technology. 68:15021512.

Liu L, Barber AH, Nuriel S, Wanger HD. 2005. Mechanical properties of functionalized single-walled carbon nanotunes/poly(vinyl alcohol) nanocomposites. Advanced functional Materials. 15: 975-980.

Ljungberg N, Cavaille JY, Heux L. 2006. Nanocomposites of isotactic polypropylene reinforced with rod-like cellulose whiskers. Polymer. 47:6285-6292.

Long D, Lequeux F. 2001. Heterogeneous dynamics at the glass transition in van der Waals liquids, in the bulk and in thin films. The European Physical Journal, E. 4:371387.

Lyons WJ. 1959. Theoretical value of the dynamic stretch modulus of cellulose. Journal of Applied Physics. 30:796-797.

Lu Y, Weng L, Cao X. 2006. Morphological, thermal and mechanical properties of ramie crystallites-reinforced plasticized starch bio composites. Carbohydrate Polymers. 63:198-204.

Mahmoud KA, Male KB, Hrapovic S, Luong HT. 2009. Cellulose nanocrystal/golf nanoparticle composites as a matrix for enzyme immobilization. Applied Materials and Interfaces. 1: 1383-1386.

Malm E, Bulone V, Wickholm K, Larsson PT, Iversen T. 2010. The surface structure of well-ordered native cellulose fibrils in contact with water. Carbohydrate Research. 345:97-100.

Mann J, Roldan-Gonzalez L. 1962. X-Ray measurements of the elastic modulus of cellulose crystals. Polymer. 3:549-553.

Marcovich NE, Auad ML, Bellesi NE, Nutt SR, Aranguren MI. 2006. Cellulose micro/nanocrystals reinforced polyurethane. Journal of Materials Research. 21:870-881.

Matsumura H, Glasser WG. 2000. Cellulose nanocomposites, II: Studies by atomic force microscopy. Journal of Applied Polymer Science. 78:2254-2261.

Matsuo M, Sawatari C, Iwai Y, Ozaki F. 1990. Effect of orientation distribution and crystallinity on the measurements by X-ray diffraction of the crystal lattice moduli of cellulose I and II. Macromolecules. 23:3266-3275. 
Maurer H, Schubert SI, Bachle F, Clauss S, Gsell D, Dual J, Niemz P. 2006. A simple anisotropy correction procedure for acoustic wood tomography. Holzforschung. 60:567573.

Mayo SC, Miller PR, Wilkins SW, Davis TJ, Gao D, Gureyev TE. 2002. Quantitative $\mathrm{X}$-ray projection microscopy: phase-contrast and multi spectral imaging. Journal of Microscopy. 207:79-96.

Mayo SC, Davis TJ, Gureyev TE, Miller PR, Paganin D, Pogany A. 2003. X-ray phase contrast microscopy and microtomography. Optic Express. 11:2289-2302.

McNeil LE, Grimsditch M. 1993. Elastic moduli of muscovite mica. Journal of Physics: Condensed Matter. 5:1681.

Mele P, Marceau S, Brown D, De Pudyt Y, Alberola ND. 2002. Reinforcement effects in fractal-structure-filled rubber. Polymer. 43:5577-5586.

Meyer KH, Lotmar W. 1936. Sur l'élasticité de la cellulose. (Sur la constitution de la partie cristallisée de la cellulose IV). Helvetica Chimica Acta. 19:68.

Mai K, Mader E, Muhle M. 1998. Interphase characterization in composites with new non-destructive methods. Composites, Part A. 29:1111-1119.

Mi Y, Zhang X, Zhou S, Cheng J, Liu F, Zhu H, Dong X, Jiao Z. 2007. Morphological and mechanical properties of bile salt modified multi-walled carbon nanotube/poly(vinyl alcohol) nanocomposites. Composites. 38(9):2041-2046.

Miller RE, Shilkrot LE, Curtin WA. 2004. A coupled atomistic and discrete dislocation plasticity simulation of nanoindentation into single crystal thin films. Acta Materialia. 52:271-284.

Miyake K, Satomi N, Sasaki S. 2006. Elastic modulus of polystyrene film for near surface to bulk measured by nanoindentation using atomic force microscopy. Applied physics Letters. 89:031925-1-3.

Miyamoto T, Takahashi S, Ito H, Inagaki H. 1989. Tissue biocompatibility of cellulose and its derivatives. Journal of Biomedical Materials Research. 23:125-133.

Mohanty AK, Khan MA, Sahoo S, and Hinrichsen G. 2000. Effect of chemical modification on the performance of biodegradable jute yarn-biopol (R) composites. Journal of Materials Science. 35: 2589-2595.

Mohanty AK, Misra M, Drzal LT. 2001. Surface modifications of natural fibers and performance of the resulting biocomposites: An overview. Composote Interfaces. 8:313343.

Moon RJ, Martini A, Nairn J, Simonsen J, Youngblood J. 2011. Cellulose nanomaterials review: structure, properties and nanocomposites. Chemical Society Reviews. 40: 3941-3994. 
Myers GR, Paganin DM, Gureyev TE, Mayo SC. 2008. Phase contrast tomography of single material objects from few projections. Optics Express. 16:908-919.

Nagy PM, Aranyi D, Horvath P, Potschke P, Pegel S, Kalman E. 2006. Nanoindentation investigation of carbon nanotube-polymer composites. Internet Electronic Journal of Molecular Design. 5:135-143.

Nakagaito AN, Iwamoto S, Yano H. 2005. Bacterial cellulose: the ultimate nanoscalar cellulose morphology for production of high strength composites. Applied Physics. 80:93-97.

Nakamae K, Nishino T, Hata K, T Matsumoto. 1986. Temperature dependence of the elastic modulus of crystalline regions of polyethylene in the direction perpendicular to the chain axis. Kobunshi Ronbunshu. 42:881-888.

Nishino T, Takano K, Nakamae K. 1995. Elastic modulus of the crystalline regions of cellulose polymorphs. Journal of Polymer Science. 33:1647-1651.

Nishiyama Y, Langan P, Chanzy H. 2002. Crystal structure and hydrogen-bonding system in cellulose I $\beta$ from synchrotron X-ray and neutron fiber diffraction. Journal of American Chemical Society. 124: 9074-9082.

Nishiyama Y, Sugiyama J, Chanzy H, Langan P. 2003. Crystal structure and hydrogen bonding system in cellulose I $\alpha$ from synchrotron $\mathrm{X}$-ray and neutron fiber diffraction. Journal of American Chemical Society. 125: 14300-14306.

Nixon WC. 1955. High resolution X-ray projection microscopy. Proceedings of the Royal Society of London. Series A, Mathematical and Physical Sciences. 232:475-485.

Noorani S, Simonsen J, Atre S. 2007. Nanoenabled microtechnology: Polysulsone nanocomposites incorporating cellulose nanocrystals. Cellulose. 14:577-584.

Ohler B. 2009. Practical advice on determination of cantilever spring constants. Bruker Application Note.

Oksman K, Mathew AP, Bondeson D, Kvien I. 2006. Manufacturing process of cellulose whiskers/polylactide acid nanocomposites. Composites Science and Technology. 66:2776-2784.

Oksman K, Sain M. 2006. Cellulose Nanocomposites: Processing, characterization and properties. American Chemical Society.

Oliver WC, Pharr GM. 1992. An improved technique for determining hardness and elastic modulus using load and displacement sending indentation experiments. Journal of Materials Research. 7:1564-1583.

Orts W J, Shey J, Imam SH, Glenn GM, Guttman ME, Revol J. 2005. Application of cellulose microfibrils on polymer nanocomposites. Journal of Polymers and the Environment. 13:301-306. 
Oyne ML, Cook RF. 2003. Load-sidplacement behavior during sharp indentation of viscus-elastic-plastic materials. Journal of Materials Research. 18:139-150.

Palaci I, Fedrigo S, Brune H, Klinke C, Chen M, Riedo E. 2005. Radial elasticity of multi-walled carbon nanotubes. Physical Review Letters. 94:175502.

Paralikar SA, Simonsen J, Lombardi J. 2008. Poly (vinyl alcohol)/cellulose nanocrystal barrier membranes. Journal of Membrane Science. 320:248-258.

Pecora R. 1985. Dynamic light scattering: applications of photon correlation spectroscopy. New York: Plenum Press.

Peijs T, van Vught RJM Govaert LE. 1995. Mechanical properties of poly(vinyl alcohol) fibers and composites. Composites. 26:83-90.

Peng Z, Xue LX, Li SD. 2005. Dynamic mechanical analysis of polyvinylalcohol/silica nanocomposite. Synthetic Metals. 152(n1-3): 25-28.

Petersoon L, Kvien I, Oksman K. 2007. Structure and thermal properties of poly(lactic acid)/cellulose whiskers nanocomposite materials. Composites Science and Technology. 67:2535-2544.

Pittenger B, Erina N, Chanmin S. 2009. Quantitative mechanical mapping at nanoscale with peak force QNM. Bruker Application Notes.

Qua EH, Hornsby PR. 2011. Preparation and characterization of nanocellulose reinforced polyamide-6. Plastics, Rubber and Composites. 40: 300-306.

Raghavan D, Gu X, Nguyen T, VanLandingham M, Karim A. 2000. Macromolecules. Mapping polymer heterogeneity using atomic force microscopy phase imaging and nanoscale indentation. 33:2573-2583.

Ranby BG. 1951. The colloidal properties of cellulose micelles. Discussions of the Faraday Society. 11:158-164.

Roman M, Winter WT. 2006. Cellulose nanocrystals; from discovery to application. TAPPI International conference on Nanotechnology. Atlanta, Georgia.

Roohani M, Habibi Y, Belgacem NM, Ebrahim G, Naghi Karimi A, Dufresne A. 2008. Cellulose Whiskers reninforced polyvinyl alcohol copolymers nanocomposites. Euoropean Polymer Journal. 44: 2489-2498.

Russ JC, Dehoff RT. 2000. Practical Stereology. New York, New York: Kluwer Academic/Plenum Publishers.

Rusli R, Eichhorn SJ. 2008. Determination of the stiffness of cellulose nanowhiskers and the fiber-matrix interface in a nanocomposite using Raman spectroscopy. Applied Physics Letters. 93:033111. 
Rusli R, Shanmuhanathan K, Rowan SJ, Weder C, Eichhorn SJ. 2011. Stress transfer in cellulose nanowhiskers composites-influence of whisker aspect ratio and surface charge. Biomacromolecules. 12: 1263-1369.

Saito T, Satoshi K, Nishiyama Y, Isogai A. 2007. Cellulose nanofibers prepared by TEMPO-mediated oxidation of native cellulose. Biomacromoleculs. 8:2485-2491.

Saito T, Shibata I, Isogai A, Suguri N, Sumikawa N. 2005. Distribution of carboxylate groups introduced into cotton linters by TEMP-mediated oxidation. Carbohydrate Polymers. 81:414-419.

Sakurada I, Ito T, Nakamae K. 1964. Elastic moduli of polymer crystals for the chain axial direction. Macromolecular Chemistry and Physics. 75:1-10.

Sakurada I, Nukushina Y, Ito T. 1962. Experimental determination of the elastic modulus of the crystalline regions in oriented polymers. Journal of Polymer Science. 57:651-660.

Schmidt H, Krug H, Kasemann R, Tiefensee F. 1991. Development of optical waveguides by sol-gel techniques for laser patterning Proceedings of SPIE. The International Society for Optical Engineering. 1590:36-43.

Schmedlen R, Masters K, West J. 2002. Photocrosslinkable polyvinyl alcohol hydrogels that can be modified with cell adhesion peptides for the use in tissue engineering. Biomaterials. 23:4325-4332.

Shibata M, Oyamada S, Kobayashi S, Yaginuma D. 2004. Mechanical composites and biodegragability of green composites based on biodegradable polyesters and lyocell fabric. Journal of Applied Polymer Science. 92:3857-3863

Shin MK, Kim SI, Kim SJ. 2006. Size-dependent elastic modulus of single electroactive polymer nanofibers. Applied Physics Letters. 89:231929.

Shojaie A, Xiaoling Li. 1997. Mechanicsm of buccal mucoadhesion of novel copolymers of acrylic acid and polyethylene glycol monimethylether monomethacrylate. Journal of Controlled Release. 47:151-161.

Singha AS, Kaith BS, Khanna AJ. 2011.Synthesis and characterization of cannabis indica fiber reinforced composites. BioResources. 6: 2101-2117.

Siqueira G, Bras J, Dufresne A. 2009. Cellulose whiskers vs. microfibrils: influence of the nature of the nanoparticle and its surface functionalization on the thermal and mechanical properties of nanocomposites. Biomacromolecules. 10:425-432.

Sneddon IN. 1965. The relation between load and penetration in the axisymmetric boussinwsq problem for a punch of arbitrary profile. International Journal of Engineering Science. 3: 47-57. 
Song J, Tranchida D, Vancso GJ. 2008. Contact mechanics of UV/Ozone-treated PDMS by AFM and JKR testing: Mechanical performance from nano- to micrometer length scales. Macromolecules. 41:6757-6762.

Sturcova A, Davies GR, Eichhorn SJ. 2005. Elastic modulus and stress-transfer properties of tunicate cellulose whiskers. Biomacromolecules. 6:1055-1061

Sun L, Han RPS, Wang J, Lim CT. 2008. Modeling the size-dependent elastic properties of polymeric nanofibers. Nanotechnology. 19:455906.

Szymanska-Chargot M, Cybulska J, Zdunek A. 2011. Sensing the structural differences in cellulose from apple and bacterial cell wall materials by Raman and FT-IR spectroscopy. Sensors. 11: 5543-5560.

Tan EPS, Lim CT. Physical properties of single polymeric nanofibers. 2004. Applied Physics Letters. 84:1603-1606.

Tan S, Sherman Jr. RL, Ford WT. 2004. Nanoscale compression of polymer microspheres by atomic force microscopy. Langmuir. 20:7015-7020.

Tanaka F, Iwata T. 2006. Estimation of the elastic modulus of cellulose crystal by molecular mechanics simulation. Cellulose. 13:509-517.

Taniguchi T, Okamura K. 1998. New films produced from microfibrillated natural fibres. Polymer International. 47:291-294.

Tashiro K, Kobayashi M. 1985. Calculation of crystallite modulus of native cellulose. Polymer Bulletin. 14: 213-218.

Tashiro K, Kobayashi M. 1991. Theoretical evaluation of three-dimensional elastic constants of native and regenerated cellulose: role of hydrogen bonds. Polymer. 32:15161526.

Tashiro K, Tadokoro H. 1981. Calculation of three-dimensional elastic constants of polymwe crystals 3. $\alpha$ and $\gamma$ forms of Nylon 6. Macromolecules. 14:781-785.

Terech P, Chazeau L, Cavaille JY. 1999. A small-angle scattering study of cellulose whiskers in aqueous suspensions. Macromolecules. 32:1872-1875.

Thomason JL, Dwight DW. 1999. The use of XPS for characterization of glass fibre coatings. Composites, Part A. 30:1401-1413.

Tokoh C, Takabe K, Fujita M, Saiki H. 1998. CP/MAS C-13 NMR and electron diffraction Study of bacterial cellulose structure affected by cell wall polysaccharides. Cellulose. 5: 249-261.

Tranchida D, Piccarolo S. 2005. Relating morphology to nanoscale mechanical properties: from crystalline to mesomorphic iPP. Polymer. 46: 4032-4040. 
Tranchida D, Piccarolo S, Joachim L, Alexeev A. 2007. Mechanical characterization of polymers on a nanometer scale through nanoindentation. A study of pile-up and viscoelasticity. Macromolecules. 40:1259-1267.

Tranchida D, Piccarolo S, Soliman M. 2006. Nanoscale mechanical characterization of polymers by AFM: Critical approach to elastic characterization. Macromolecules. 39:4547-4556.

Treloar LRG. 1960. Calculation of elastic moduli of polymer crystals: III. Cellulose. Polymers. 1:290-303.

Tserki V, Matzinos P, Kokkou S, Panayiotou C. 2005. Novel biodegradable composites based on treated lignocellulosic waste flour as filler. Part I. Surface chemical modification and characterization of waste flour. Composites. 36: 965-974.

Tsukruk VV, Huang A, Chizhik SA, Gorbunov VV. 1998. Probing of micromechanical properties of compliant polymeric mareials. Journal of Materials Science. 33:4905-4909.

Turbark A, Snyder F, Sandberg K. 1983. Microfibrillated cellulose, a new cellulose product: Properties, uses and commercial potential. Journal of Applied Polymer Science. 37:815-827.

Uskokovic PS, Tang CY, Tsui CP, Ignjatovic N, Uskokovic DP. 2007. Micromechanical properties of hydroxyapetite/poly-L-lactide biocomposite using nanoindentation and modulus mapping. Journal of European Ceramic Society. 27: 15591564.

Vaia RA, Ishii H, Giannelis EP. 1996. Synthesis and properties of 2D nanostructures by direct intercalation of polymer melts in layered silicates. Chemistry of Materials. 5:1694-1696.

Vaia RA, Liu WD. 2002. X-ray powder diffraction of polymer/layered silicate nanocomposites: model and practice. Journal of Polymer Science, Polymer Physics. 40:1590-1600.

Vanlandingham MR, Villarrubia JS, Guthrie WF, Meyers GF. 2001. Nanoindentation of polymers: An overview. Macromolecular Symposia. 167:15-43.

VanLandingham MR, McKnight SH, Palmese GR, Elings JR, Huang X, Bogetti TA, Edulhee RF, Gillespie JW. 1997. Nanoscale indentation of polymer systems using the atomic force microscope. Journal of Adhesion. 64:31-59.

Wagberg L, Decher G, Norgren M, Lindstrom T, Ankerfors M, Axnas K. 2008. The built-up of polyelectrolyte multilayers of microfibrillated cellulose and cationic polyelectrolytes. Langmuir. 24: 784-795. 
Wan W, Campbell G, Zhang Z, Hui A, Boughner D. 2002. Optimizing the tensile properties of poly(vinyl alcohol) hydrogel for the construction of a bioprosthetic heart valve stent. Journal of Biomedical Material Research. 63:854-86

Wan YZ, Hong SRJ, Huang Y, Zhu Y, Wang YL, Jiang HJ. 2006. Synthesis and characterization of hydroxyapetite-bacterial cellulose nanocomposites. Composites Science and Technology. 66: 1825-1832.

Wan YZ, Huang Y, Yuan CD, Raman S, Zhu Y, Jiang HJ, He F, Gao C. 2007. Biomimetic synthesis of hydroxyapetite/bacterial cellulose nanocomposites for biomedical applications. Materials Science and Engineering. 27: 855-864.

Wang Y, Cao X, Zhang L. 2006. Effect of cellulose whiskers on properties of soy protein thermoplastics. Macromolecular Bioscience. 6:524-531.

Wang B, Sain M. 2007. Isolation of nanofibers from soybean source and their reinforcing capability on synthetic polymers. Composites Science and Technology. 67:2521-2527.

Wang B, Sain M. 2007. Dispersion of soybean stock-based nanofiber in a plastic matrix. Polymer International. 56:538-546.

Weiss P, Nihouannen D Le, Rau C, Pilet P, Aguado E, Gaitheir O, Jean A, Daculsi G. 2005. Synchrotron and non synchrotron X-ray microtomography three-dimesional representation of bone in-growth in calcium phosphate biomaterials. European Cells and Materials. 9:48-49.

Williams JG, Donnellan ME, James MR, Morris WL. 1990. Properties of the interphase in organic matrix composites. Material Science and Engineering. 126:305-312.

Winter RM, Houston JE. 1998. Interphase mechanical properties in epoxy-glass Fiber composites as measured by interfacial microscopy. Proceedings of the Society for Experimental Mechanics. Spring Conference on Experimental and Applied Mechanics. 355-358.

Wong S, Kasapis S, Tan YM. 2009. Bacterial and plant cellulose modification using ultrasound irradiation. Carbohydrate Polymers. 77: 280-287.

Wung CJ, Yang Y, Prasat PN, Karasz FE. 1991. Poly(p-phenylene vinylene)-silica composite. A novel sol-gel processed non-linear optical material for optical waveguides. Polymer. 32:605-608.

Yachi T, Hayashi J, Takai M, Shimizu Y. 1983. Supermolecular structure of cellulose: stepwise decrease in LODP and particle size of cellulose hydrolyzed after chemical treatment. Journal of Applied polymer Science, Applied Polymer Symposium. 37:325343.

Yang S, Zhang Y. 2004.Analysis of nanoindetation creep for polymeric materials. Journal of Applied Physics. 95:3655-3666. 
Yongshang L, Weng L, Cao X. 2005. Morphological, thermal and mechanical properties of ramie crystallites-reinforced plasticized starch biocomposites. Carbohydrate Polymers. 63:198-204.

Zhang CY, Zhang YW, Zeng KY, Shen L. 2005. Nanoindetation of polymers with sharp indenter. Journal of Materials Research. 20:1597-1605.

Zhao Y, Ge Z, Fang J. 2008. Elastic modulus of viral nanotubes. Physical Review, E. 78:031914.

Zheng X, Cao Y, li B, Feng Z, Wang G. 2010. Surface effects in various bendingbased test methods for measuring the elastic properties of nonowires. Nanotechnology. 21:205702.

Zimmermann T, Pöhler E, Geiger T. 2004. Cellulose fibrils for polymer reinforcement. Advanced Engineering Materials. 6:754-761.

Zimmermann T, Pohler E, Schwaller P. 2005.Mechanical and morphological properties of cellulose fibril reinforced nanocomposites. Advanced Engineering Materials. 7:1156-1161.

Zugenmaier P. 2001. Conformation and packing of various crystalline cellulose fibers. Progress in Polymer Science. 26:1341-1417. 


\section{Appendices}

\section{Appendix A: Atomic Force Microscopy ${ }^{6}$}

\section{A.1 Definition}

The atomic force microscope (AFM) has emerged as a useful tool for studying surface topography and forces (Binnig et al., 1986). AFM is able to image the force and topography in every kind of surface (insulator and conductive) and in every kind of environment (in air and in liquid), with high force $(1 \mathrm{pN})$, lateral $(25 \mathrm{~nm})$ and vertical $(0.1$ A) resolution. A unique feature of the system is the capability to correlate the force measurements with topography data. AFM operates by movement of a cantilever that deflects when interacting with the sample surface. The cantilever scans the surface by means of a piezoactuator. The cantilever deflection will be measured to reproduce the sample topography. The most common method to detect cantilever deflections is the optical lever method. Here a laser beam is focused on the back side of the cantilever, then the reflected beam is detectedusing a position sensor (Figure 11.1).

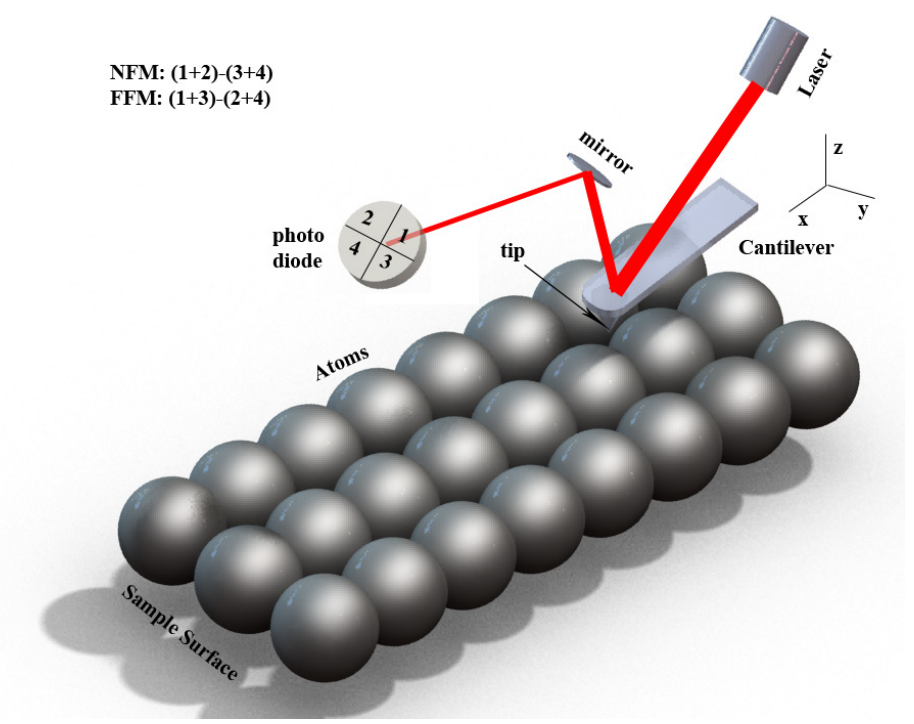

Figure 11.1 Schematic representation of an AFM. The cantilever-tip system is deflected by the surface topography of the sample. Cantilever deflections are detected with a laseroptical set-up. The four-segment photodiode detects normal forces (normal force microscopy (NFM)) and frictional forces (FFM) affecting the tip. In most AFM systems the sample rests on a piezotube scanner (not shown in this figure) which allows a scanning motion in $x$ - and $y$-directions as well as movement in $z$-direction (Reprinted from Surface Science, 491 (3), KD Jandt, Atomic force microscopy of biomaterilas surfaces and interfaces, 303-332, 2001 with permission from Elsivier) .

6 The material presented in this chapter has been submitted for publication in Encyclopedia of Tribology. See Appendix C for proper documentations. 


\section{A.2 Scientific Fundementals}

AFM typically operates in three distinct modes: (a) contact mode, (b) non-contact mode, and (c) tapping mode. In the contact mode, the tip is in continues contact with the surface of the sample, while in the tapping mode, the tip oscillates at a tip amplitude of several tens of nanometers. The frequencies of oscillation in air is $50-500 \mathrm{kHz}$ and in the fluids approximately $10 \mathrm{kHz}$. In the non-contact the cantilever tip oscillates about 50-150 $A$ above the sample surface to detect the surface forces acting between the tip and the sample.

The interaction forces between the tip and sample in all of the three open-loop modes can be distinctly identified on a force-displacement curve as shown in Figure 11.2. In short distances above the sample surface, the van der Waals forces are dominant and the nature of force interactive. By decreasing this distance, the attractive force increases until the AFM tip contacts the surface. At this position the attractive force is maximum. The nature of this force transforms to repulsive as the distance becomes even smaller. The repulsive force is due to the interaction of electron clouds on the AFM tip and the sample surface.

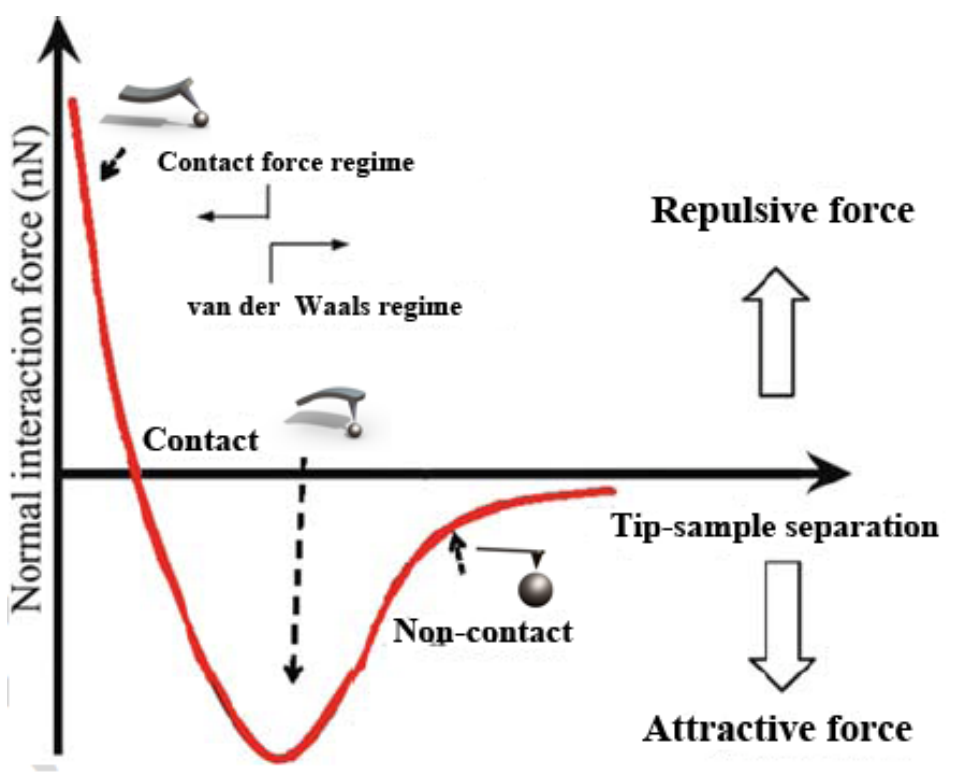

Figure 11.2 Interatomic force variation versus the distance between AFM tip and sample.

\section{A.3 Mechanical Characterization of Surfaces in AFM}

In addition to angstrom resolution imaging capacity, due to its very fine force sensing capabilities and ultra small tip radius, AFM has also been used as a powerful tool for characterizing the mechanical properties of various surfaces. Burnham and Colton (Burnham and Colton 1986) were among the first who proposed a method to use an AFM like instrument for directly measuring nanomechanical properties of materials. A 
common approach is based on the phase imaging in the tapping mode. Phase imaging is a qualitative technique, and extracting precise quantitative mechanical data from these images is impractical because the resonant behavior of the cantilever also acts as a filter.

Many other techniques have been developed to overcome this incompetence and they mostly include acquiring the force on the tip versus its vertical position at a specific point on the sample. In order to generate a map of the properties across a larger sample, force volume imaging was developed (Radmacher et al. 1994), which acquires force curves at each pixel in an image. Because force volume imaging is very slow, pulsed force mode (Rosa-Zeiser et al. 1997) was developed which is faster and uses a relatively fast sinusoidal ramping. Other techniques including HarmoniX (Pittenger 2009) and most recently PeakForce tapping (Pittenger et al. 2009) have been developed toward the direction of fully quantitative characterization of surface mechanics.

\section{A.3.1 Fundamentals of AFM Force Measurements}

A main application of AFM is to measure the surfaces forces. In this technique, the vertical position of the piezo $\left(Z_{p}\right)$ and the deflection of the cantilever $\left(Z_{c}\right)$ are recorded as the tip approaches and retracts the sample. The parameter $Z_{c}$ is mostly measured by optical lever technique, where a beam of laser is focused on the end of the cantilever (where the tip is), and the position of the reflected beam is monitored by a position sensitive detector (Meyer 1988). When a normal force $(F)$ is applied the cantilever bends and the position of reflected beam changes on the detector. The deflection of a cantilever $(\mathrm{E}=$ elastic modulus) with rectangular cross section of thickness $(t)$, width $(w)$ and length $(L)$ is given by $Z_{c}=\frac{4 F L^{3}}{E W t^{3}}$. Knowing the spring constant of the cantilever $\left(k_{c}\right)$, the force is calculated by $F=k_{c} Z_{c}$. In Figure 11.3, the distance $(D)$ is simply the total of the position of the piezo and the cantilever deflection $\left(D=Z_{c} Z_{p}\right)$.
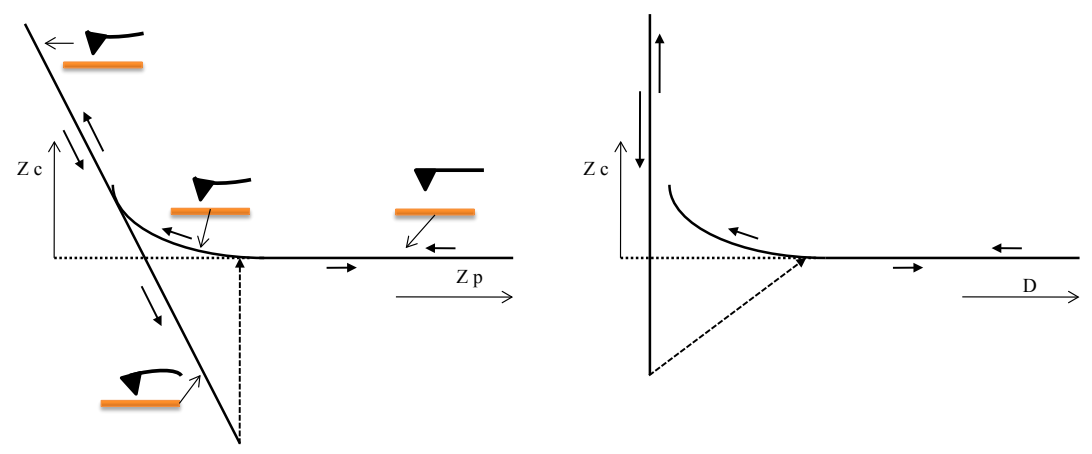

Figure 11.3 Schematic of a common Left: cantilever deflection versus piezo height and Right: corresponding cantilever deflection versus distance.

\section{A.3.2 Cantilevers}

Cantilevers are one of the key elements in mechanical measurements by AFMs. Properties of cantilevers are largely responsible for their performances and can affect the measurements greatly. Some of the criteria for selection of appropriate cantilever for 
mechanical testing include (i) the mechanical properties of the cantilevers. Mechanics of the cantilever is defined by its spring constant $K_{c}$ and resonance frequency $v_{0}$ (Butt 2005). For a rectangular cantilever ( $\rho$ is the density): $K=\frac{F}{Z_{c}}=\frac{E w t^{3}}{4 L^{3}}$ and $v_{0}=0.1615 \frac{t}{L^{2}} \sqrt{\frac{E}{\rho}}$. Appropriate cantilever should have high sensitivity in $Z_{c}$ direction, which is achieved by low spring constants and it should have high resonance frequency so that external vibrations and noises are less transmitted to the cantilever and fast scans are achievable; (ii) High lateral stability to avoid twisting during the scans; (iii) the surface properties of cantilevers. The selection of highly reflective surfaces increases the signal detected at the position sensitive detector; (iv) Tip geometry and particularly the radius of the tip.

\section{A.3.3 The Contact Regime}

The contact part of the force curves, both in approach and retraction, contains information on the elastic properties of the sample (e.g. Young's modulus). There are several theories which describe the elastic deformation of the sample under a defined load $(F)$, these theories vary in their description of the contact radius $(a)$ or the deformation $(\delta)$ due to their different assumptions made for the adhesion forces in the system. Three of most commonly used theories for a spherical particle on a plane surface are summarized in Table 11.6 (Hertz (1882), Johnson-Kendall-Roberts (JKR) (Johnson et al. 1971), Derjaguin-Muller-Toporov (DMT) (Derjaguin et al. 1975).

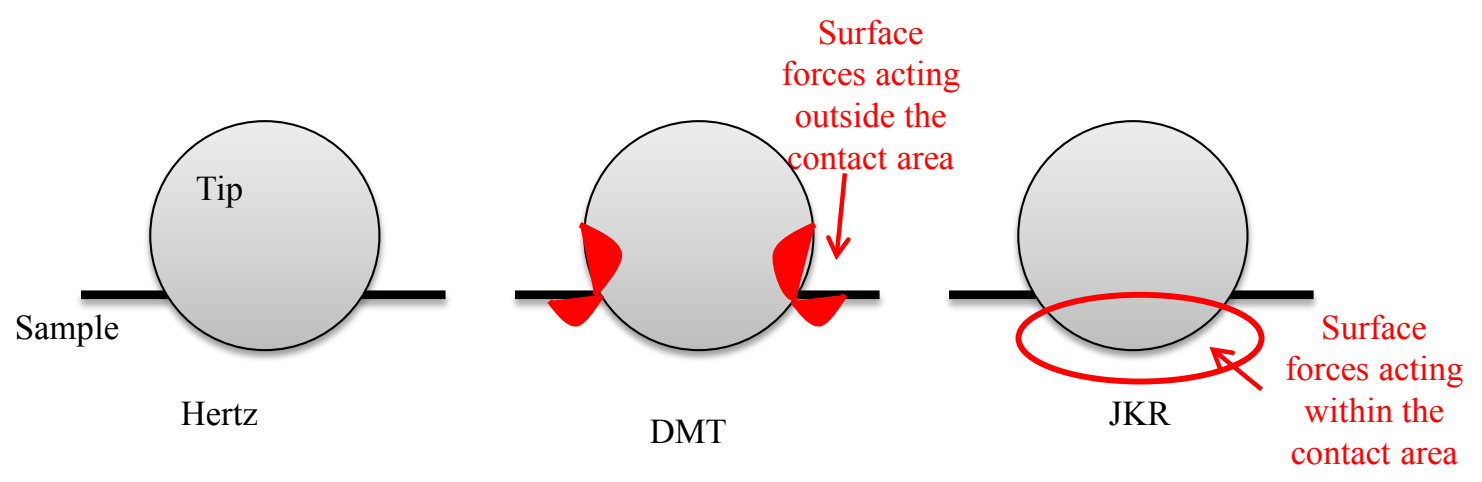

Figure 11.4 Schematics of Hertz, JKR and DMT models for the surface forces at the contact regime (rigid tip and compliant substrate).

In DMT model, the main assumption is the presence of long-range attractive forces present outside of the contact area (shown in red color in Figure 11.4), whereas Hertz consider no surface and adhesion force and JKR only assumes short-range attractive forces local at the contact area. Typically DMT works the best for small radius tips and rigid materials with low surface energy. JKR model is a better fit for compliant and high surface energy materials. Hertz model is simpler and could be applied for cases where negligible surface forces exist. 
Table 11.1

Hertz, JKR and DMT models for the contact regime. (contact radius $=a$, sample deformation $=\delta$, adhesion force $=F_{a d}$, tip radius $=R$, Adhesion work per unit area $=W$, applied force $=F$, reduced Young's modulus $=\mathrm{E}_{\text {red }}$ )

\begin{tabular}{|c|c|c|c|}
\hline & Hertz & DMT & JKR \\
\hline Assumptions & $\begin{array}{l}\text {-No surface } \\
\text { forces } \\
\text {-No adhesion }\end{array}$ & $\begin{array}{l}\text {-Model geometry } \\
\text { as in Hetrz model } \\
\text {-Long range } \\
\text { attractive forces } \\
\text { present outside the } \\
\text { contact region }\end{array}$ & $\begin{array}{l}\text {-Geometry } \mathrm{f} \text { contact area is } \\
\text { deformable } \\
\text {-Short range attractive forces } \\
\text { local at the contact area }\end{array}$ \\
\hline $\begin{array}{l}\text { Best } \\
\text { describes }\end{array}$ & $\begin{array}{l}\text {-Negligible } \\
\text { surface forces } \\
\text {-High applied } \\
\text { loads }\end{array}$ & $\begin{array}{l}- \text {-Small tips } \\
\text {-Rigid and low } \\
\text { surface energy } \\
\text { materials }\end{array}$ & $\begin{array}{l}\text {-large tips } \\
\text {-Compliant and high surface } \\
\text { energy materials }\end{array}$ \\
\hline Limitations & $\begin{array}{l}\text { Low loads and } \\
\text { the presence of } \\
\text { adhesion }\end{array}$ & $\begin{array}{l}\text { Underestimates the } \\
\text { contact area }\end{array}$ & Underestimates the loading \\
\hline$a$ & $\sqrt[3]{\frac{R F}{E_{\text {red }}}}$ & $\sqrt[3]{\frac{R(F+2 \pi R W)}{E_{\text {red }}}}$ & $\sqrt[3]{\frac{R\left(F+3 \pi R W F+(3 \pi R W)^{2}\right.}{E_{\text {red }}}}$ \\
\hline$\delta$ & $\begin{array}{l}\frac{a^{2}}{R} \\
=\left(\frac{F^{2}}{R E_{r e d}^{2}}\right)^{1 / 3}\end{array}$ & $\begin{array}{l}\frac{a^{2}}{R} \\
=\frac{(F+2 \pi R W)^{2 / 3}}{\sqrt[3]{R E_{r e d}^{2}}}\end{array}$ & $\frac{a^{2}}{R}=\frac{2}{3} \sqrt{\frac{6 \pi W a}{E_{\text {red }}}}$ \\
\hline$F_{a d}$ & 0 & $2 \pi \mathrm{RW}$ & $3 \pi \mathrm{RW} / 2$ \\
\hline
\end{tabular}

\section{A.4 Key Applications in Surface Mechanics}

The force sensing of surfaces has opened up tremendous development in understanding the details of surface science and adhesion. Several examples are given below:

Measurement of fundamental forces: electrostatic, capillary and van der Wals force measurements (Butt 1991), measurements in liquids (Ducker et al. 1992), quantized adhesion (Hoh et al. 1992), colloidal forces (Ducker et al. 1991), friction (Overney et al. 1992)

Biological applications: binding forces (Dammer et al. 1995), biomechanical measurements (Wojcikiewicz et al. 2004), single molecule experiments (Hugel et al. 2001)

Material science applications: Nanoscale mechanics (Hues et al. 1993), polymer and organic films (Mizes et al. 1991; Overney et al. 1994), clusters and single crystals (Hues 
et al. 1994), indentation and plastic deformation (Sumomogi et al. 1994; Bhushan and Koinkar 1994)

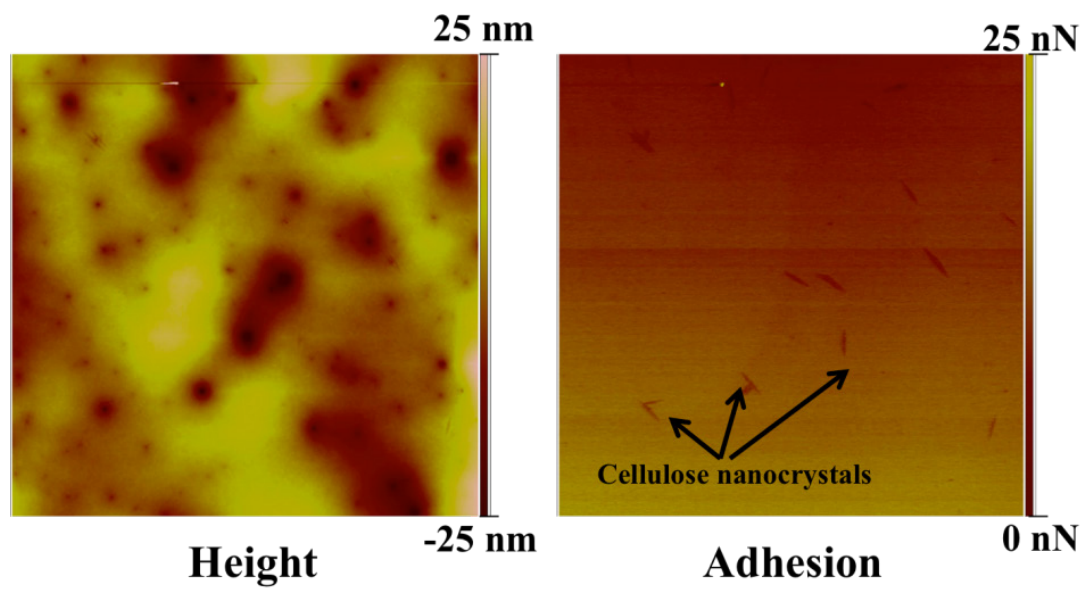

Figure 11.5 The height image and adhesion maps of a cellulose nanocomposite are shown. The nanocrystals are embeded in the polymer matrix and are not detectable in the height image. However, the adhesion map obtained by peak force AFM mode detects the presence of nanocrystals marked by arrows. The nanocrystals have lower adhesion force to the AFM canteliver in compasirson to the polymer matrix. The adhesion map is quantified for values up to $25 n N$.

\section{A.5 Electrical Characteriztion in AFM}

Characterization of local properties of surfaces materials at micro-, nano- and atomic scales can be performed using several techniques including electric force microscopy (EFM), scanning capacitance microscopy (SCM), Kelvin probe force microscopy (KPFM), and piezoresponse force microscopy (PFM) are some of the SPM techniques which were developed for characterization of local electric properties of materials. In this section, some of main SPM techniques that are used for characterization of local electric properties of materials are reviewed. Because of space limitation, review of all electric characterization techniques is not possible here. However, some of the relevant references are mentioned in each section.

\section{A.5.1 Electric Force Microscopy (EFM)}

In this technique the conductive AFM tip scans the surface and electrostatic forces will be mapped across the surface of the sample (Martin et al. 1988). The electric field induced by trapped charges or applying an electric voltage between tip and sample can be measured. Electric force microscopy can be operated in three different operation modes: amplitude detection, phase detection and phase modulation.

A schematic picture of the EFM setup is shown in Figure 11.6. The simplest EFM setup consists of a metal-coated cantilever which goes near the sample surface and measuring the mechanical resonance frequency of the cantilever as a function of the voltage applied between the sample and cantilever. Equations of motion for a voltage-driven cantilever are as follows: 


$$
\begin{gathered}
u=u_{0} \\
z=z_{0}+A \\
A=A_{0}(\omega) \exp (i(\omega t-\theta)) \\
g=0
\end{gathered}
$$

where $u, z$, and $g$ show position of piezo, tip, and sample, respectively. In equations (11.4-7) $A_{0}$ and $w$ are the amplitude and oscillation frequency of the tip, and $g$ is a phase angle.

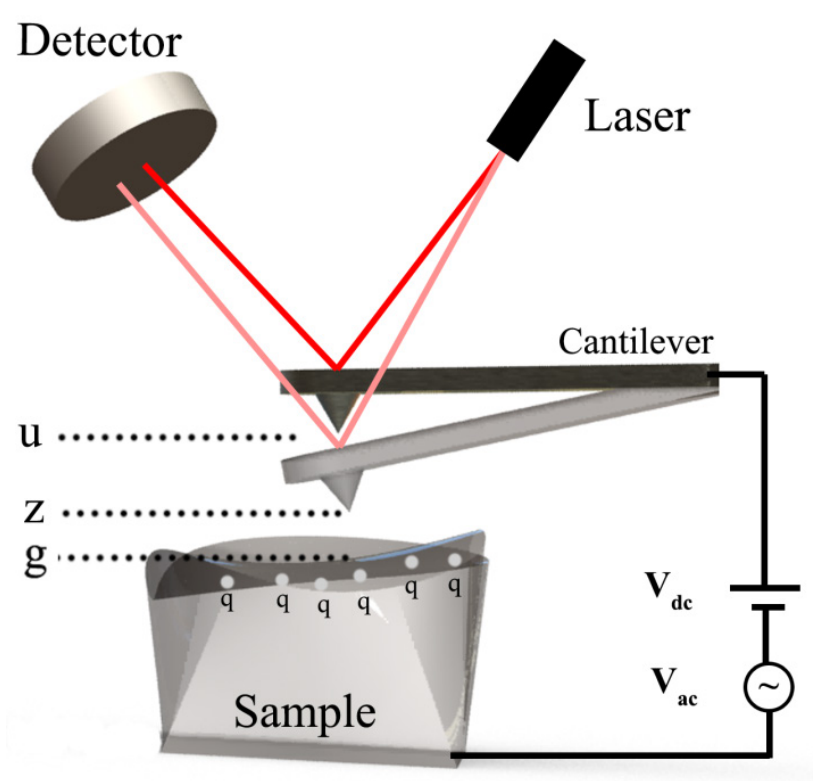

Figure 11.6 A schematic of electric force microscopy (EFM) is shown. A variable and a constant voltage source are connected in series between the tip and the sample. Deflection of the cantilever will be measured by laser beam and a position sensitive detector (PSD). Electric field due to the presence of charges, $q$, can be detected by change in the amplitude or oscillation phase of the cantilever.

The electric field which is due to charges on the sample applies an external force to the cantilever. Gradient of applied external force changes the resonance frequency of the cantilever which will be measured using a feedback control system. Attractive gradient equivalents to additional spring in tension attached to the tip which reduces the cantilever resonance frequency. However, repulsive gradient equivalents to additional spring in compression attached to the tip which increases cantilever's resonance frequency.

It can be shown that vibration amplitude of cantilever at effective vibration frequency, $\omega=\omega_{0}^{\prime}$ is given by:

$$
A_{r}\left(\omega_{0}^{\prime}\right)=A Q \sqrt{K / K^{\prime}}
$$


where $Q, k$ and $k$ are quality factor, stiffness and effective stiffness of cantilever, respectively. Derivative of the applied electric force can be calculated directly using the following relation:

$$
\frac{\partial F}{\partial z}=2 K \frac{\delta \omega}{\omega_{0}}
$$

where $\delta \omega$ is the shift in the frequency of oscillation of cantilever. Variation in force derivative changes the amplitude of vibration. Working at the steepest slope of resonance curve, the variation in amplitude of oscillation can be calculated as follows:

$$
\delta A=\frac{2}{3 \sqrt{3}} \frac{Q}{K} A_{r} \delta\left(F_{d c}^{\prime}+F_{\Omega}^{\prime}+F_{2 \Omega}^{\prime}\right)
$$

where $F_{\mathrm{dc}}, F_{\Omega}, F_{2 \Omega}$ are the force derivative due to constant voltage source and alternating voltage source at $\omega$ and $2 \omega$ frequencies, respectively. Force derivatives depend on the capacitance between the top and sample, magnitude of constant and oscillating voltages, and sample's surface charge. Details of derivation of these equations can be found in (Sarid et al. 1994).

A,5.2 Scanning capacitance microscopy (SCM) and Kelvin Probe Force Microscopy (KPFM)

Scanning capacitance microscopy is a non-contact mode AFM technique. A schematic picture of SCM is shown in Figure 11.7. In this technique a conductive tip is brought in close proximity of the surface, about $20 \mathrm{~nm}$ above the surface, and scans the sample (Matey and Blanc. 1985). An alternating voltage is applied between the tip and the sample which induces changes in capacitance. This technique has a wide range of applications such as dopant-profiling, failure analysis of semiconductor devices, imaging of device operation, and visualization of radiation effects.

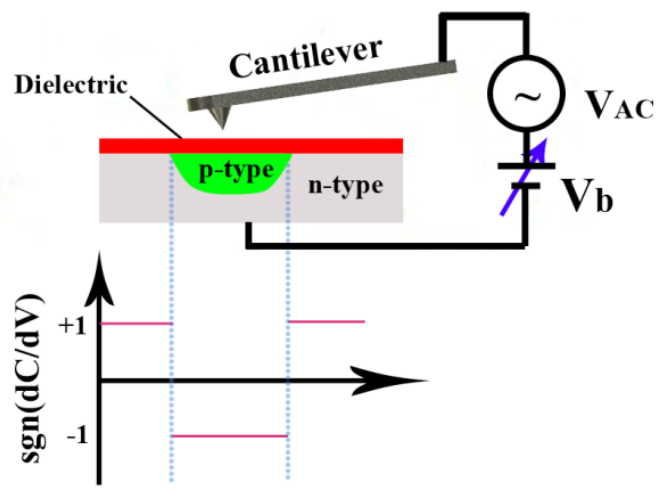

Figure 11.7 A schematic of scanning capacitance microscope (SCM) is shown. Type of the dopant can be determined by the sign of $(d C / d V)$, which is positive for n-typed dopant and negative for p-typed dopant. Dopant concentration is inversely related to $d C / d V$, i.e. higher concentration results in smaller $d C / d V$, and vice versa. 
In the SCM technique, the force which is applied to cantilever tip due to the applied electric voltage is (Paul 2001):

$$
F=\frac{1}{2} d C / d z V^{2}
$$

The applied voltage consists of two terms, constant dc voltage and variable ac voltage, $V=V_{\mathrm{dc}}+V_{\mathrm{ac}} \cdot \sin (\omega t)$. Substituting $V$ in equation (11.11), and decomposing the force based on the applied frequency:

$$
\begin{gathered}
F_{d c}=\frac{1}{2} \frac{d C}{d z}\left[V_{d c}^{2}+\frac{1}{2} V_{a c}^{2}\right] \\
F_{\omega}=\frac{d C}{d z} V_{d c} V_{a c} \sin (\Omega \mathrm{t}) \\
F_{2 \omega}=-\frac{1}{4} \frac{d C}{d z} V_{a c}^{2} \cos (2 \omega t)
\end{gathered}
$$

For SCM the lock-in amplifier monitors the signal with $2 \omega$ frequency which is used for calculating the capacitance between sample and tip. The constant voltage $V_{\mathrm{dc}}$ consists of three terms as follows:

$$
V_{d c}=V_{b}+V_{c p}+V_{\text {induced }}
$$

which $V_{\mathrm{b}}, V_{\mathrm{cp}}$, and $V_{\text {induced }}$ representing bias voltage of the constant voltage source, contact potential and externally induced voltage, respectively. If a closed loop controller changes $V_{\mathrm{b}}$ such that $F_{\omega}=0$, then the surface potential can be mapped. This technique is known as Kelvin probe force microscopy (KPFM).

\section{A.5.3 Piezoresponse Force Microscopy (PFM)}

PFM works based on the inverse piezoelctric effect. Inverse piezoelectric response of the material refers to the phenomena in which an applied electric field induces mechanical strain in the piezoelectric material. If the applied electric field is parallel to the material polarization, the material expands, while it contracts if the electric field is anti-parallel.

A schematic figure of PFM is shown in Figure 11.8. In PFM an oscillating electric potential will be applied to a conductive AFM tip that is contact with the sample surface and the deflection of the cantilever will be monitored using a lock-in amplifier. For electric fields parallel to material's polarization the cantilever oscillates in phase with the applied electric field, and out of phase for anti-parallel polarization domains. One should note that the alternative voltage has a frequency far from resonance frequency of the cantilever (less than one-third of cantilever's natural frequency) to avoid driving the cantilever to its mechanical oscillation (Gruverman 2003). The phase difference between oscillation of cantilever and alternative voltage shows the difference in the angle of polarization vector and electric field. 

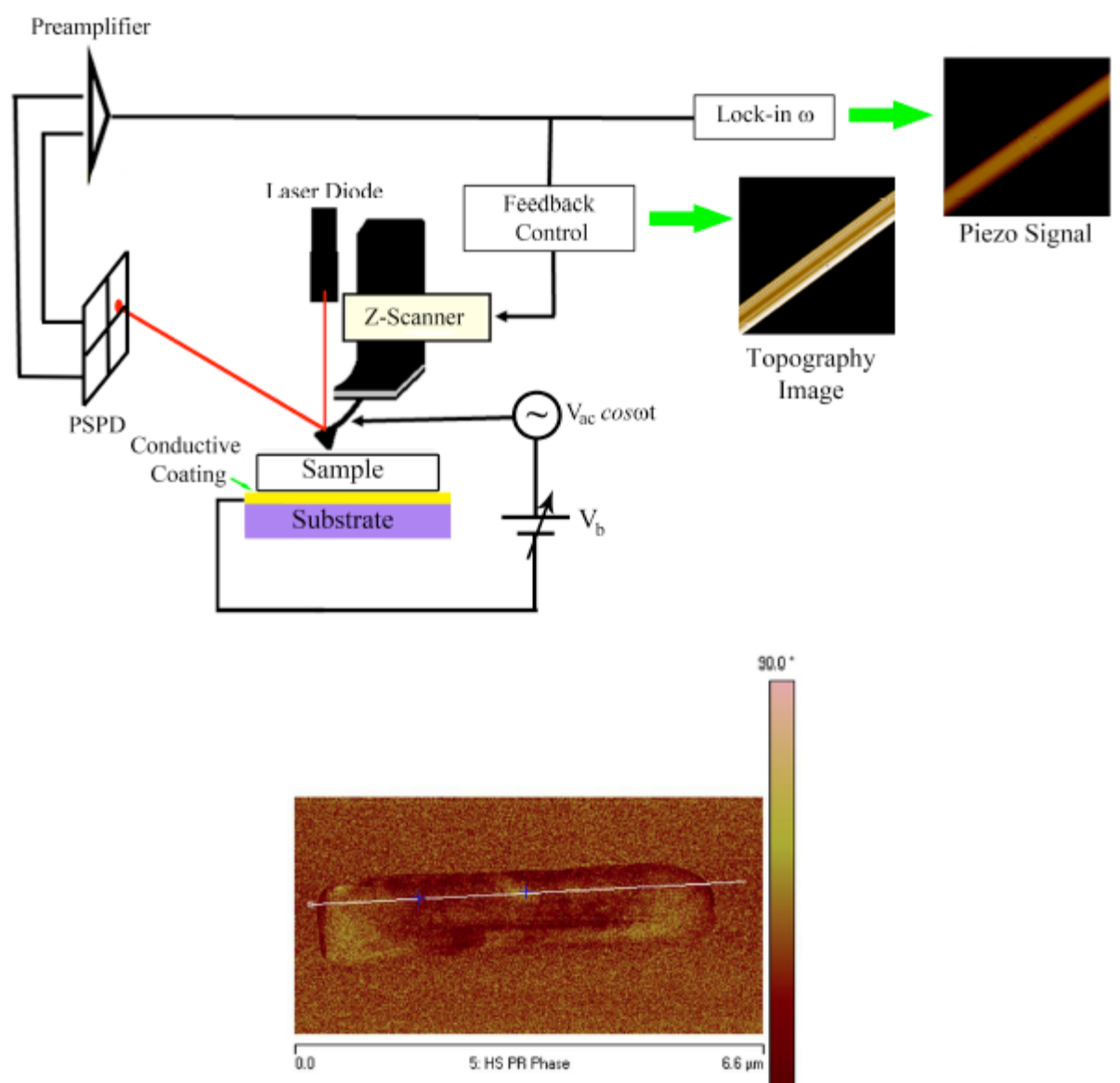

(a)

Figure 11.8 (Top) Schematic diagram of the PFM is shown. There is an alternative voltage source (Vac cos $\omega \mathrm{t}$ ) between the conductive AFM tip and conductive substrate. Deflection of the cantilever will be monitored by position sensitive photo detector (PSPD). The feedback control on z-height gives the topography image while the lock-in amplifier provides piezo-response image. (Down) Phase image of piezorsponse of a $\mathrm{ZnO}$ nanobelt shows domains along the axis of nanobelt.

There are two approaches for exciting the piezoelectric sample, local excitation and integral excitation. In the local excitation approach the piezoelectric sample is excited locally by applying an alternative voltage between the tip and bottom electrode. Local excitation enables the monitoring of the local piezoelectric response of the sample, but the electric field in this case is highly non-uniform. In the integral excitation approach a top electrode will be coated on the sample. In this case the electric field that is formed in the material would be homogenous which allows quantitative measurements of the piezoelectric response of the sample. 
In PFM the piezoelectric material vibrates to the applied modulating electric voltage, $V=V_{a c} \cdot \cos (\omega t)$ with a vibration amplitude, $Z_{0}$, same frequency and phase difference $\varphi$. Therefore, it can be shown as:

$$
\Delta Z=Z_{0} \cos (\omega t+\phi)
$$

For piezoelectric domains parallel to the applied electric field, $\varphi=0$, and for anti-parallel domains, $\varphi=180^{\circ}$. The measured piezoelectric signal is sensitive to the applied electric signal. For small forces electrostatic interactions between the cantilever and bottom electrode of the sample is not negligible. However, for large interactions the contribution of electric force is negligible compared to the piezoelectric force (Kalinin and Bonnell 2002).

Deflection of cantilever has a contribution from capacitive force between the cantilever and bottom electrode in addition to the piezoeresponse of the sample. The capacitive force applied can be calculated as (Kholkin 2007):

$$
F_{\text {cap }}=\frac{1}{2} V^{2} \frac{d C}{d z}
$$

where $V$ is the applied voltage, and $C$ is the capacitance of AFM tip/sample/bottom electrode capacitor. Capacitance of the system can be written as the sum of capacitance of cantilever, $C_{\text {cant }}$, and capacitance of the tip, $C_{\text {tip. }}$. Capacitance of the cantilever can be estimated by relation (Prume 2002):

$$
\frac{d C_{c a n t}}{d z}=\frac{\varepsilon_{0} d_{c}}{\tan \alpha}\left(\frac{1}{h_{c}}-\frac{1}{L_{c} \tan \alpha+h_{c}}\right)
$$

where $d_{\mathrm{c}}, L_{\mathrm{c}}, h_{\mathrm{c}}$, and $a$ are width, length of the cantilever, the gap and angle between the cantilever and the sample surface, respectively. There is also an attractive Coulomb force between the charged ferroelectric sample and the tip. This force can be estimated using the equation proposed by (Hong et al. 1998):

$$
F_{\text {coul }}=\frac{\sigma C V}{2 \varepsilon_{0}}
$$

where $\sigma$ is the surface-bound charge density and $V$ is the voltage applied to the sample.

\section{A.6 Key Applications of Electrical AFM}

Conductive-AFM methods are widely used for the characterization of inorganic semiconductors and electrical properties of organic materials has been demonstrated. The examples include local electrical characteristics of composite materials. For instance, a blend of two semiconducting polymers exhibited clear differences in the local I-V characteristics allowing the distinction of various phases (Alexeev et al. 2006). Electrical characteristics of AFM can also be used for the analysis of contact resistance and the correlation with surface wear (Bhushan et al. 2008). Piezoelectric property characterization of thin films is another application for CAFM. The magnitude of the effective longitudinal piezoelectric constant $\left(d_{33}\right)$ of $\mathrm{ZnO}$ thin films (Christman 1998) and other thin films have been measured. 


\section{A.7 References}

Alexeev A, Loos J, Koetse MM. 2006. Nanoscale electrical characterization of semiconducting polymer blends by conductive atomic force microscopy (C-AFM). Ultramicroscopy. 106: 191-199

Binnig G, Quate CE, Gerber Ch. 1986. Atomic force microscope. Physics Review Letters. 56: 930

Bhushan B, Koinkar VN. 1994. Nanoindentation hardness measurements using atomic force microscopy. Applied Physics letters. 64: 1653

Bhushan B, Palacio M, Kinzig B. 2008. AFM-based nanotribological and electrical characterization of ultrathin wear-resistant ionic liquid films. Journal of Colloid and Interface Science. 317: 275-287

Burnham NA, Colton RJ. 1989. Measuring the nanomechanical properties and surface forces of m,aterials using an atomic force microscope. Journal of Vacuum Science \& Technology. 7: 2906

Butt HJ. 1991. Measuring electrostatic, van der Waals, and hydration forces in electrolyte solutions with an atomic force microscope. Biophysical Journal. 60: 1438

Butt HJ, Cappella B, Kappel M. 2005.Force measurements with the atomic force microscope technique, interpretation and applications. Surface Science Reports. 59:1

Christman JA, Woolcott Jr. RR, Kingon AI, Nemanich RJ. 1998. Piezoelectric measurements with atomic force microscopy. Applied Physics Letters. 73:3851-3853

Dammer U, Popescu O, Wagner P, Anselmetti D, Guntherodt HJ, Misevic GN. 1995. Binding strength between cell adhesion proteoglycans measured by atomic force microscopy. Science. 267: 1173

Derjaguin BV, Muller VM, Toporov YP. 1975. Effect of contact deformations on adhesion. Journal of Colloid and Interface Science. 53: 314

Ducker W, Senden T, Pashley R. 1991. Direct measurement of colloidal forces using an atomic force microscope. Nature. 353: 239

Ducker W, Senden T, Pashley R. 1992. Measurement of forces in liquids using a force microscope, Langmuir. 8: 1831

Gruverman A, Auciello O, Tokumoto H. 2003. Imaging and control of domain structures in ferroelectric thin films via scanning force microscopy. Annual Review of Materials Science. 28: 101-123.

Hertz H. 1882. Uber die Beruhrung fester elasticher Korper. Journal of Reine Angew Math. 92: 156 
Hoh JH, Cleveland JP, Prater CB, Revel JP, Hansma PK. 1992. Quantized adhesion detected with the atomic force microscope. Journal of the American Chemical Society. 114: 4917

Hong JW. 1998. Detection and control of ferroelectric domains by an electrostatic force microscope, Journal of Vacuum Science \& Technology B: Microelectronics and Nanometer Structures. 16: 2942-2946.

Hues SM, Colton RJ, Meyer E, Guntherodt HJ. 1993. Scanning probe microscopy of thin films. MRS Bulletin. 18: 41

Hues SM, Draper CF, Colton RJ. 1994. Measurement of nanomechanical properties of metals using the atomic force microscope. Journal of Vacuum Science \& Technology B: Microelectronics and Nanometer Structures. 12: 2211

Jandt KD. 2001. Atomic force microscopy of surfaces and interfaces. Surface Science. 491: 303

Kholkin A, Roelfs A, Kalinin S, Gruverman A. 2007. Review of Ferroelectric Domain imaging by piezoresponse force microscopy. Scanning Probe Microscopy. 173-214.

Kalinin SV, Bonnell DA. 2002. Imaging mechanism of piezoresponse force microscopy of ferroelectric surfaces. Physical Review B 65(12): 125408.

Martin Y, Abraham DW, Wickramasinghe HK. 1988. High-resolution capacitance measurement and potentiometry by force microscopy. Applied Physics Letters. 52(13): 1103-1105

Matey JR, Blanc J. 1985. Scanning capacitance microscopy. Journal of Applied Physics. 57(5): 1437-1444.

Paul G. 2001. Electrostatic force microscopy: principles and some applications to semiconductors. Nanotechnology. 12: 485

Prume K, Roelofs A, Schmitz T, Reichenberg B, Tiedke S, Waser R. 2002. Compensation of the Parasitic Capacitance of a Scanning Force Microscope Cantilever Used for Measurements on Ferroelectric Capacitors of Submicron Size by Means of Finite Element Simulations. Japanese Journal of Applied Physics. 41: 7198

Sarid D. 1994. Scanning force microscopy with applications to electric, magnetic, and atomic forces. Revised edition. Oxford: Oxford University Press. 


\section{Appendix B: Copyright Permissions}

\section{B.1 Permission for Figures Used in Chapter 1}

Figure 1.5 Permission

Rightslink Printable License

Page 1 of 3

SPRINGER LICENSE

TERMS AND CONDITIONS

Oct 26, 2011

\begin{tabular}{|c|c|}
\hline \multicolumn{2}{|c|}{$\begin{array}{l}\text { This is a License Agreement between Anahita Pakzad ("You") and Springer ("Spring } \\
\text { provided by Copyright Clearance Center ("CCC"). The license consists of your order } \\
\text { the terms and conditions provided by Springer, and the payment terms and conditions } \\
\text { All payments must be made in full to cCc. For payment instructions, please see } \\
\text { information listed at the bottom of this form. }\end{array}$} \\
\hline License Number & 2776771009356 \\
\hline License date & Oct 26,2011 \\
\hline Licensed content publisher & Springer \\
\hline Licensed content publication & Applied Physics A \\
\hline Licensed content title & $\begin{array}{l}\text { Bacterial cellulose: the ultimate nano-scalar cellulose morphology } \\
\text { for the production of high-strength composites }\end{array}$ \\
\hline Licensed content author & A.N. Nakagaito \\
\hline Licensed content date & Jan 1, 2005 \\
\hline Volume number & 80 \\
\hline Issue number & 1 \\
\hline Type of Use & Thesis/Dissertation \\
\hline Portion & Figures \\
\hline $\begin{array}{l}\text { Author of this Springer } \\
\text { article }\end{array}$ & No \\
\hline \multicolumn{2}{|l|}{ Order reference number } \\
\hline $\begin{array}{l}\text { Title of your thesis / } \\
\text { dissertation }\end{array}$ & $\begin{array}{l}\text { Nanomechanics of Cellulose Crystals and Cellulose-Based Polymer } \\
\text { Composites }\end{array}$ \\
\hline
\end{tabular}


equal to the number that you identified in the licensing process.

This License includes use in an electronic form, provided it is password protected or on the university's intranet, destined to microfilming by UMI and University repository. For any other electronic use, please contact Springer at (permissions.dordrecht@springer.com or permissions.heidelberg@springer.com)

The material can only be used for the purpose of defending your thesis, and with a maximum of 100 extra copies in paper.

Although Springer holds copyright to the material and is entitled to negotiate on rights, this license is only valid, provided permission is also obtained from the (co) author (address is given with the article/chapter) and provided it concerns original material which does not carry references to other sources (if material in question appears with credit to another source, authorization from that source is required as well). Permission free of charge on this occasion does not prejudice any rights we might have to charge for reproduction of our copyrighted material in the future.

Altering/Modifying Material: Not Permitted

However figures and illustrations may be altered minimally to serve your work. Any other abbreviations, additions, deletions and/or any other alterations shall be made only with prior written authorization of the author(s) and/or Springer Science + Business Media. (Please contact Springer at permissions.dordrecht@springer.com or permissions.heidelberg@springer.com)

Reservation of Rights

Springer Science + Business Media reserves all rights not specifically granted in the combination of (i) the license details provided by you and accepted in the course of this licensing transaction, (ii) these terms and conditions and (iii) CCC's Billing and Payment terms and conditions.

Copyright Notice:

Please include the following copyright citation referencing the publication in which the material was originally published. Where wording is within brackets, please include verbatim. "With kind permission from Springer Science+Business Media: <book/journal title, chapter/article title, volume, year of publication, page, name(s) of author(s), figure number (s), and any original (first) copyright notice displayed with material>."

Warranties: Springer Science + Business Media makes no representations or warranties with respect to the licensed material.

Indemnity 
to any other person without Springer Science + Business Media's written permission.

No Amendment Except in Writing

This license may not be amended except in a writing signed by both parties (or, in the case of Springer Science + Business Media, by CCC on Springer Science + Business Media's behalf).

Objection to Contrary Terms

Springer Science + Business Media hereby objects to any terms contained in any purchase order, acknowledgment, check endorsement or other writing prepared by you, which terms are inconsistent with these terms and conditions or CCC's Billing and Payment terms and conditions. These terms and conditions, together with CCC's Billing and Payment terms and conditions (which are incorporated herein), comprise the entire agreement between you and Springer Science + Business Media (and CCC)

concerning this licensing transaction. In the event of any conflict between your obligations established by these terms and conditions and those established by CCC's Billing and Payment terms and conditions, these terms and conditions shall control.

Jurisdiction

All disputes that may arise in connection with this present License, or the breach thereof, shall be settled exclusively by the country's law in which the work was originally published.

Other terms and conditions:

$\mathrm{v} 1.2$

If you would like to pay for this license now, please remit this license along with your payment made payable to "COPYRIGHT CLEARANCE CENTER" otherwise you will be invoiced within 48 hours of the license date. Payment should be in the form of a check or money order referencing your account number and this invoice number RLNK500653310.

Once you receive your invoice for this order, you may pay your invoice by credit card. Please follow instructions provided at that time.

Make

Payment

To:

Copyright

Clearance

Center

Dept 001

P.O. Box 843006

Boston, MA 02284-3006

For suggestions or comments regarding this order, contact RightsLink Customer Support: customercare@copyright.com or +1-877-622-5543 (toll free in the US) or +1- 
Figure 1.6 and 1.7 Permissions

Rightslink ${ }^{\circledR}$ by Copyright Clearance Center

Page 1 of 1

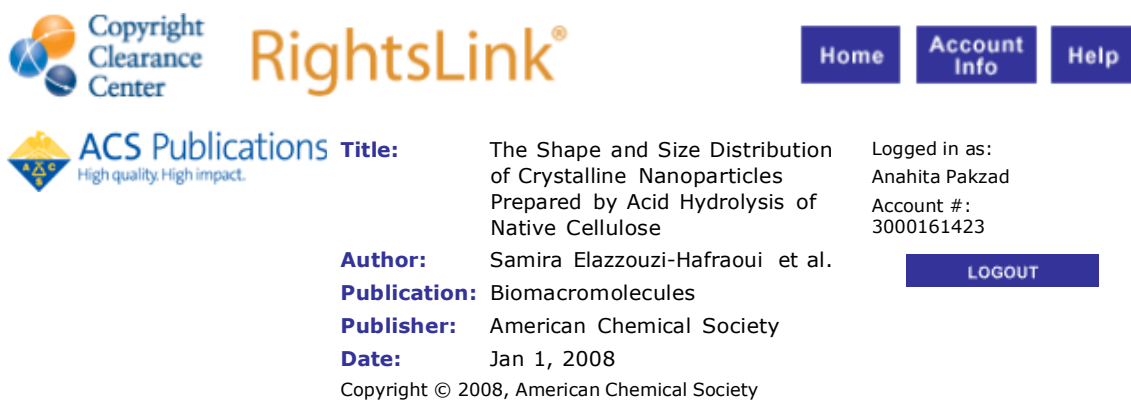

PERMISSION/LICENSE IS GRANTED FOR YOUR ORDER AT NO CHARGE

This type of permission/license, instead of the standard Terms \& Conditions, is sent to you because no fee is being charged for your order. Please note the following:

- Permission is granted for your request in both print and electronic formats.

- If figures and/or tables were requested, they may be adapted or used in part.

- Please print this page for your records and send a copy of it to your publisher/graduate school.

- Appropriate credit for the requested material should be given as follows: "Reprinted (adapted) with permission from (COMPLETE REFERENCE CITATION). Copyright (YEAR) American Chemical Society." Insert appropriate information in place of the capitalized words.

- One-time permission is granted only for the use specified in your request. No additional uses are granted (such as derivative works or other editions). For any other uses, please submit a new request.

Copyright (C) 2011 Copyright Clearance Center, Inc. All Rights Reserved. Privacy statement.

Comments? We would like to hear from you. E-mail us at customercare@copyright.com 


\section{Figure 1.12 Permission}

Rightslink Printable License

ELSEVIER LICENSE

TERMS AND CONDITIONS

Oct 27, 2011

This is a License Agreement between Anahita Pakzad ("You") and Elsevier ("Elsevier") provided by Copyright Clearance Center ("CCC"). The license consists of your order details, the terms and conditions provided by Elsevier, and the payment terms and conditions.

\begin{tabular}{|c|c|}
\hline Supplier & $\begin{array}{l}\text { Elsevier Limited } \\
\text { The Boulevard,Langford Lane } \\
\text { Kidlington,Oxford,OX5 1GB,UK }\end{array}$ \\
\hline $\begin{array}{l}\text { Registered Company } \\
\text { Number }\end{array}$ & 1982084 \\
\hline Customer name & Anahita Pakzad \\
\hline Customer address & Michigan Technological University \\
\hline & Houghton, MI 49931 \\
\hline License number & 2777350716535 \\
\hline License date & Oct 27,2011 \\
\hline Licensed content publisher & Elsevier \\
\hline Licensed content publication & Composites Science and Technology \\
\hline Licensed content title & $\begin{array}{l}\text { Isolation of nanofibers from soybean source and their reinforcing } \\
\text { capability on synthetic polymers }\end{array}$ \\
\hline Licensed content author & Bei Wang,Mohini Sain \\
\hline Licensed content date & September 2007 \\
\hline $\begin{array}{l}\text { Licensed content volume } \\
\text { number }\end{array}$ & 67 \\
\hline $\begin{array}{l}\text { Licensed content issue } \\
\text { number }\end{array}$ & $11-12$ \\
\hline Number of pages & 7 \\
\hline Start Page & 2521 \\
\hline End Page & 2527 \\
\hline Type of Use & reuse in a thesis/dissertation \\
\hline $\begin{array}{l}\text { Intended publisher of new } \\
\text { work }\end{array}$ & other \\
\hline
\end{tabular}




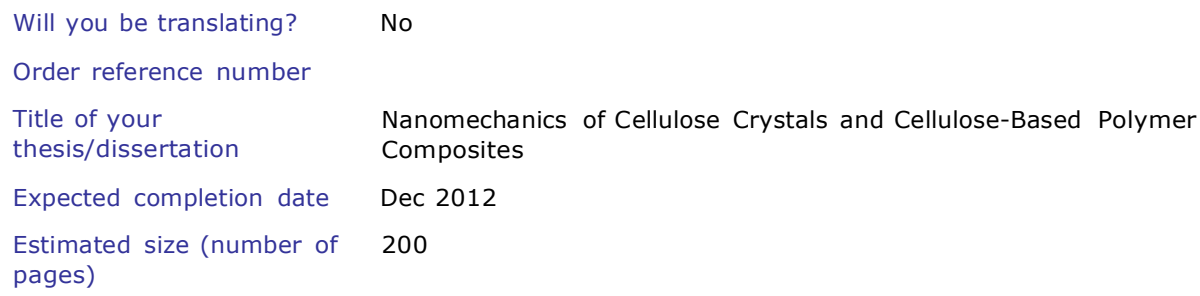

1. The publisher for this copyrighted material is Elsevier. By clicking "accept" in connection with completing this licensing transaction, you agree that the following terms and conditions apply to this transaction (along with the Billing and Payment terms and conditions established by Copyright Clearance Center, Inc. ("CCC"), at the time that you opened your Rightslink account and that are available at any time at http://myaccount.copyright.com).

\section{GEN \\ ERA \\ L \\ TER \\ MS}

2. Elsevier hereby grants you permission to reproduce the aforementioned material subject to the terms and conditions indicated.

3. Acknowledgement: If any part of the material to be used (for example, figures) has appeared in our publication with credit or acknowledgement to another source, permission must also be sought from that source. If such permission is not obtained then that material may not be included in your publication/copies. Suitable acknowledgement to the source must be made, either as a footnote or in a reference list at the end of your publication, as follows:

"Reprinted from Publication title, Vol /edition number, Author(s), Title of article / title of chapter, Pages No., Copyright (Year), with permission from Elsevier [OR APPLICABLE SOCIETY COPYRIGHT OWNER]." Also Lancet special credit - "Reprinted from The Lancet, Vol. number, Author(s), Title of article, Pages No., Copyright (Year), with permission from Elsevier."

4. Reproduction of this material is confined to the purpose and/or media for which permission is hereby given.

5. Altering/Modifying Material: Not Permitted. However figures and illustrations may be altered/adapted minimally to serve your work. Any other abbreviations, additions, deletions and/or any other alterations shall be made only with prior written authorization of Elsevier Ltd. (Please contact Elsevier at permissions@elsevier.com) 
6. If the permission fee for the requested use of our material is waived in this instance, please be advised that your future requests for Elsevier materials may attract a fee.

7. Reservation of Rights: Publisher reserves all rights not specifically granted in the combination of (i) the license details provided by you and accepted in the course of this licensing transaction, (ii) these terms and conditions and (iii) CCC's Billing and Payment terms and conditions.

8. License Contingent Upon Payment: While you may exercise the rights licensed immediately upon issuance of the license at the end of the licensing process for the transaction, provided that you have disclosed complete and accurate details of your proposed use, no license is finally effective unless and until full payment is received from you (either by publisher or by CCC) as provided in CCC's Billing and Payment terms and conditions. If full payment is not received on a timely basis, then any license preliminarily granted shall be deemed automatically revoked and shall be void as if never granted. Further, in the event that you breach any of these terms and conditions or any of CCC's Billing and Payment terms and conditions, the license is automatically revoked and shall be void as if never granted. Use of materials as described in a revoked license, as well as any use of the materials beyond the scope of an unrevoked license, may constitute copyright infringement and publisher reserves the right to take any and all action to protect its copyright in the materials.

9. Warranties: Publisher makes no representations or warranties with respect to the licensed material.

10. Indemnity: You hereby indemnify and agree to hold harmless publisher and CCC, and their respective officers, directors, employees and agents, from and against any and all claims arising out of your use of the licensed material other than as specifically authorized pursuant to this license.

11. No Transfer of License: This license is personal to you and may not be sublicensed, assigned, or transferred by you to any other person without publisher's written permission.

12. No Amendment Except in Writing: This license may not be amended except in a writing signed by both parties (or, in the case of publisher, by $\mathrm{CCC}$ on publisher's behalf).

13. Objection to Contrary Terms: Publisher hereby objects to any terms contained in any purchase order, acknowledgment, check endorsement or other writing prepared by you, which terms are inconsistent with these terms and conditions or CCC's Billing and Payment terms and conditions. These terms and conditions, together with CCC's Billing and Payment terms and 
or Copyright Clearance Center be responsible or liable for any costs, expenses or damage incurred by you as a result of a denial of your permission request, other than a refund of the amount(s) paid by you to Elsevier and/or Copyright Clearance Center for denied permissions.

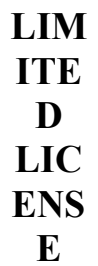

The following terms and conditions apply only to specific license types:

15. Translation: This permission is granted for non-exclusive world English rights only unless your license was granted for translation rights. If you licensed translation rights you may only translate this content into the languages you requested. A professional translator must perform all translations and reproduce the content word for word preserving the integrity of the article. If this license is to re-use 1 or 2 figures then permission is granted for non-exclusive world rights in all languages.

16. Website: The following terms and conditions apply to electronic reserve and author websites:

Electronic reserve: If licensed material is to be posted to website, the web site is to be password-protected and made available only to bona fide students registered on a relevant course if:

This license was made in connection with a course, This permission is granted for 1 year only. You may obtain a license for future website posting, All content posted to the web site must maintain the copyright information line on the bottom of each image,

A hyper-text must be included to the Homepage of the journal from which you are licensing at http://www.sciencedirect.com/science/journal/xxxxx or the Elsevier homepage for books at http://www.elsevier.com, and Central Storage: This license does not include permission for a scanned version of the material to be stored in a central repository such as that provided by Heron/XanEdu.

17. Author website for journals with the following additional clauses:

All content posted to the web site must maintain the copyright information line on the bottom of each image, and he permission granted is limited to the personal version of your paper. You are not allowed to download and post the published electronic version of your article (whether PDF or 
18. Author website for books with the following additional clauses:

Authors are permitted to place a brief summary of their work online only.

A hyper-text must be included to the Elsevier homepage at http://www.elsevier.com

All content posted to the web site must maintain the copyright

information line on the bottom of each image

You are not allowed to download and post the published electronic version of your chapter,

nor may you scan the printed edition to create an electronic version.

Central Storage: This license does not include permission for a

scanned version of the material to be stored in a central repository

such as that provided by Heron/XanEdu.

19. Website (regular and for author): A hyper-text must be included to the Homepage of the journal from which you are licensing at

http://www.sciencedirect.com/science/journal/xxxxx. or for books to the Elsevier homepage

at http://www.elsevier.com

20. Thesis/Dissertation: If your license is for use in a thesis/dissertation your thesis may be submitted to your institution in either print or electronic form. Should your thesis be published commercially, please reapply for permission. These requirements include permission for the Library and Archives of Canada to supply single copies, on demand, of the complete thesis and include permission for UMI to supply single copies, on demand, of the complete thesis. Should your thesis be published commercially, please reapply for permission.

\section{Other Conditions:}

v1.6

If you would like to pay for this license now, please remit this license along with your payment made payable to "COPYRIGHT CLEARANCE CENTER" otherwise you will be invoiced within 48 hours of the license date. Payment should be in the form of a check or money order referencing your account number and this invoice number RLNK500654076.

Once you receive your invoice for this order, you may pay your invoice by credit card. Please follow instructions provided at that time.

Make

Payment

To:

Copyright

Clearance 
Figure 1.13 Permission

Rightslink ${ }^{\circledR}$ by Copyright Clearance Center

Page 1 of 1

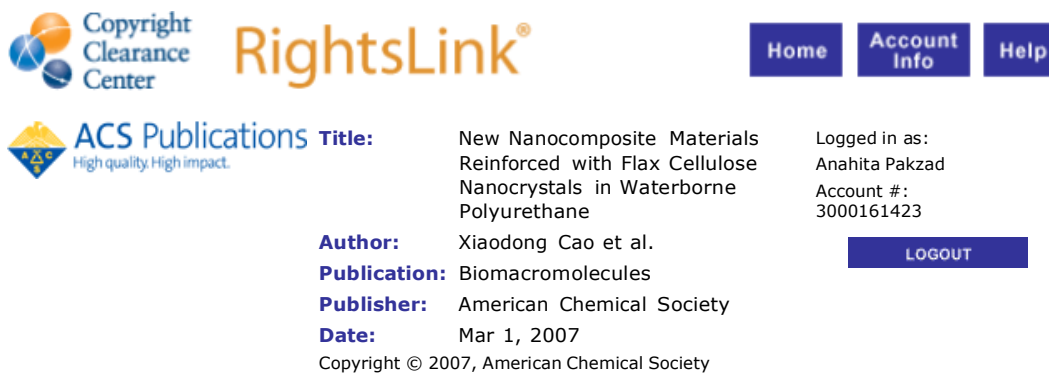

PERMISSION/LICENSE IS GRANTED FOR YOUR ORDER AT NO CHARGE

This type of permission/license, instead of the standard Terms \& Conditions, is sent to you because no fee is being charged for your order. Please note the following:

- Permission is granted for your request in both print and electronic formats.

- If figures and/or tables were requested, they may be adapted or used in part.

- Please print this page for your records and send a copy of it to your publisher/graduate school.

- Appropriate credit for the requested material should be given as follows: "Reprinted (adapted) with permission from (COMPLETE REFERENCE CITATION). Copyright (YEAR) American Chemical Society." Insert appropriate information in place of the capitalized words.

- One-time permission is granted only for the use specified in your request. No additional uses are granted (such as derivative works or other editions). For any other uses, please submit a new request.

Copyright (c) 2011 Copyright Clearance Center, Inc. All Rights Reserved. Privacy statement. Comments? We would like to hear from you. E-mail us at customercare@copyright.com 
Figure 1.14 Permission

Rightslink ${ }^{\circledR}$ by Copyright Clearance Center

Page 1 of 1

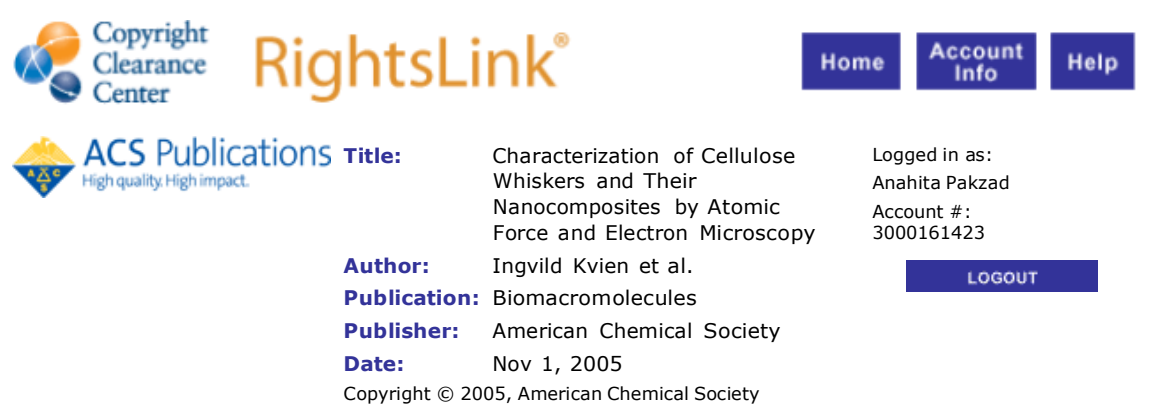

PERMISSION/LICENSE IS GRANTED FOR YOUR ORDER AT NO CHARGE

This type of permission/license, instead of the standard Terms \& Conditions, is sent to you because no fee is being charged for your order. Please note the following:

- Permission is granted for your request in both print and electronic formats.

- If figures and/or tables were requested, they may be adapted or used in part.

- Please print this page for your records and send a copy of it to your publisher/graduate school.

- Appropriate credit for the requested material should be given as follows: "Reprinted (adapted) with permission from (COMPLETE REFERENCE CITATION). Copyright (YEAR) American Chemical Society." Insert appropriate information in place of the capitalized words.

- One-time permission is granted only for the use specified in your request. No additional uses are granted (such as derivative works or other editions). For any other uses, please submit a new request.

\section{BACK CLOSE WINDOW}

Copyright @ 2011 Copyright Clearance Center, Inc. All Rights Reserved. Privacy statement. Comments? We would like to hear from you. E-mail us at customercare@copyright.com 


\section{B.2 Chapter 3 Permission}

This is a License Agreement between Anahita Pakzad ("You") and John Wiley and Sons ("John Wiley and Sons") provided by Copyright Clearance Center ("CCC"). The license consists of your order details, the terms and conditions provided by John Wiley and Sons, and the payment terms and conditions.

All payments must be made in full to CCC. For payment instructions, please see information listed at the bottom of this form.

\begin{tabular}{ll} 
License Number & 2740380845223 \\
License date & Sep 01, 2011 \\
Licensed content publisher & John Wiley and Sons \\
Licensed content publication & Journal of Microscopy \\
Licensed content title & $\begin{array}{l}\text { Revealing the 3D internal stru} \\
\text { microcomposites using X-ray }\end{array}$ \\
Licensed content author & A. PAKZAD,N. PARIKH,P.A. \\
Licensed content date & Jul 1, 2011 \\
Start page & 77 \\
End page & 85 \\
Type of use & Dissertation/Thesis \\
Requestor type & Author of this Wiley article \\
Format & Print and electronic \\
Portion & Full article \\
Will you be translating? & No \\
Order reference number & \\
Total & 0.00 USD \\
Terms and Conditions & \\
\hline
\end{tabular}

TERMS AND CONDITIONS

This copyrighted material is owned by or exclusively licensed to John Wiley \& Sons, Inc. or one of its group companies (each a "Wiley Company") or a society for whom a Wiley Company has exclusive publishing rights in relation to a particular journal (collectively WILEY"). By clicking "accept" in connection with completing this licensing transaction, you agree that the following terms and conditions apply to this transaction (along with the billing and payment terms and conditions established by the Copyright Clearance Center Inc., ("CCC's Billing and Payment terms and conditions"), at the time that you opened your Rightslink account (these are available at any time at http://myaccount.copyright.com)

Terms and Conditions

1. The materials you have 
2. You are hereby granted a personal, non-exclusive, non-sublicensable, nontransferable, worldwide, limited license to reproduce the Materials for the purpose specified in the licensing process. This license is for a one-time use only with a maximum distribution equal to the number that you identified in the licensing process. Any form of republication granted by this licence must be completed within two years of the date of the grant of this licence (although copies prepared before may be distributed thereafter). The Materials shall not be used in any other manner or for any other purpose. Permission is granted subject to an appropriate acknowledgement given to the author, title of the material/book/journal and the publisher. You shall also duplicate the copyright notice that appears in the Wiley publication in your use of the Material.

Permission is also granted on the understanding that nowhere in the text is a previously published source acknowledged for all or part of this Material. Any third party material is expressly excluded from this permission.

3. With respect to the Materials, all rights are reserved. Except as expressly granted by the terms of the license, no part of the Materials may be copied, modified, adapted (except for minor reformatting required by the new Publication), translated, reproduced, transferred or distributed, in any form or by any means, and no derivative works may be made based on the Materials without the prior permission of the respective copyright owner. You may not alter, remove or suppress in any manner any copyright, trademark or other notices displayed by the Materials. You may not license, rent, sell, loan, lease, pledge, offer as security, transfer or assign the Materials, or any of the rights granted to you hereunder to any other person.

4. The Materials and all of the intellectual property rights therein shall at all times remain the exclusive property of John Wiley \& Sons Inc or one of its related companies (WILEY) or their respective licensors, and your interest therein is only that of having possession of and the right to reproduce the Materials pursuant to Section 2 herein during the continuance of this Agreement. You agree that you own no right, title or interest in or to the Materials or any of the intellectual property rights therein. You shall have no rights hereunder other than the license as provided for above in Section 2. No right, license or interest to any trademark, trade name, service mark or other branding ("Marks") of WILEY or its licensors is granted hereunder, and you agree that you shall not assert any such right, license or interest with respect thereto.

5. NEITHER WILEY NOR ITS LICENSORS MAKES ANY WARRANTY OR REPRESENTATION OF ANY KIND TO YOU OR ANY THIRD PARTY, EXPRESS, IMPLIED OR STATUTORY, WITH RESPECT TO THE MATERIALS OR THE ACCURACY OF ANY INFORMATION CONTAINED IN THE MATERIALS, INCLUDING, WITHOUT LIMITATION, ANY IMPLIED WARRANTY OF MERCHANTABILITY, ACCURACY, SATISFACTORY QUALITY, FITNESS FOR A PARTICULAR PURPOSE, USABILITY, INTEGRATION OR NON-INFRINGEMENT AND ALL SUCH WARRANTIES ARE HEREBY EXCLUDED BY WILEY AND ITS LICENSORS AND WAIVED BY YOU.

6. WILEY shall have the right to terminate this Agreement immediately upon breach of this Agreement by you.

7. You shall indemnify, defend and hold harmless WILEY, its Licensors and their respective directors, officers, agents and employees, from and against any actual or threatened claims, demands, causes of action or proceedings arising from any breach of this Agreement by you. 
thereby.

10. The failure of either party to enforce any term or condition of this Agreement shall not constitute a waiver of either party's right to enforce each and every term and condition of this Agreement. No breach under this agreement shall be deemed waived or excused by either party unless such waiver or consent is in writing signed by the party granting such waiver or consent. The waiver by or consent of a party to a breach of any provision of this Agreement shall not operate or be construed as a waiver of or consent to any other or subsequent breach by such other party.

11. This Agreement may not be assigned (including by operation of law or otherwise) by you without WILEY's prior written consent.

12. Any fee required for this permission shall be non-refundable after thirty (30) days from receipt.

13. These terms and conditions together with CCC's Billing and Payment terms and conditions (which are incorporated herein) form the entire agreement between you and WILEY concerning this licensing transaction and (in the absence of fraud) supersedes all prior agreements and representations of the parties, oral or written. This Agreement may not be amended except in writing signed by both parties. This Agreement shall be binding upon and inure to the benefit of the parties' successors, legal representatives, and authorized assigns.

14. In the event of any conflict between your obligations established by these terms and conditions and those established by CCC's Billing and Payment terms and conditions, these terms and conditions shall prevail.

15. WILEY expressly reserves all rights not specifically granted in the combination of (i) the license details provided by you and accepted in the course of this licensing transaction, (ii) these terms and conditions and (iii) CCC's Billing and Payment terms and conditions.

16. This Agreement will be void if the Type of Use, Format, Circulation, or Requestor Type was misrepresented during the licensing process.

17. This Agreement shall be governed by and construed in accordance with the laws of the State of New York, USA, without regards to such state's conflict of law rules. Any legal action, suit or proceeding arising out of or relating to these Terms and Conditions or the breach thereof shall be instituted in a court of competent jurisdiction in New York County in the State of New York in the United States of America and each party hereby consents and submits to the personal jurisdiction of such court, waives any objection to venue in such court and consents to service of process by registered or certified mail, return receipt requested, at the last known address of such party.

\section{Wiley Open Access Terms and Conditions}

All research articles published in Wiley Open Access journals are fully open access: immediately freely available to read, download and share. Articles are published under the terms of the Creative Commons Attribution Non Commercial License. which permits use, distribution and reproduction in any medium, provided the original work is properly cited and is not used for commercial purposes. The license is subject to the Wiley Open Access terms and conditions:

Wiley Open Access articles are protected by copyright and are posted to repositories and 
and data-mine the content subject to the following conditions:

- The authors' moral rights are not compromised. These rights include the right of "paternity" (also known as "attribution" - the right for the author to be identified as such) and "integrity" (the right for the author not to have the work altered in such a way that the author's reputation or integrity may be impugned).

- Where content in the article is identified as belonging to a third party, it is the obligation of the user to ensure that any reuse complies with the copyright policies of the owner of that content.

- If article content is copied, downloaded or otherwise reused for non-commercial research and education purposes, a link to the appropriate bibliographic citation (authors, journal, article title, volume, issue, page numbers, DOI and the link to the definitive published version on Wiley Online Library) should be maintained. Copyright notices and disclaimers must not be deleted.

- Any translations, for which a prior translation agreement with Wiley has not been agreed, must prominently display the statement: "This is an unofficial translation of an article that appeared in a Wiley publication. The publisher has not endorsed this translation."

\section{Use by commercial "for-profit" organisations}

Use of Wiley Open Access articles for commercial, promotional, or marketing purposes requires further explicit permission from Wiley and will be subject to a fee. Commercial purposes include:

- Copying or downloading of articles, or linking to such articles for further redistribution, sale or licensing;

- Copying, downloading or posting by a site or service that incorporates advertising with such content;

- The inclusion or incorporation of article content in other works or services (other than normal quotations with an appropriate citation) that is then available for sale or licensing, for a fee (for example, a compilation produced for marketing purposes, inclusion in a sales pack)

- Use of article content (other than normal quotations with appropriate citation) by forprofit organisations for promotional purposes

- Linking to article content in e-mails redistributed for promotional, marketing or educational purposes;

- Use for the purposes of monetary reward by means of sale, resale, licence, loan, transfer or other form of commercial exploitation such as marketing products

- Print reprints of Wiley Open Access articles can be purchased from:

corporatesales@wiley.com

Other Terms and Conditions:

BY CLICKING ON THE "I AGREE..." BOX, YOU ACKNOWLEDGE THAT YOU

HAVE READ AND FULLY UNDERSTAND EACH OF THE SECTIONS OF AND

PROVISIONS SET FORTH IN THIS AGREEMENT AND THAT YOU ARE IN AGREEMENT WITH AND ARE WILLING TO ACCEPT ALL OF YOUR OBLIGATIONS AS SET FORTH IN THIS AGREEMENT.

v1.7

If you would like to pay for this license now, please remit this license along with your 
payment made payable to "COPYRIGHT CLEARANCE CENTER" otherwise you will be invoiced within 48 hours of the license date. Payment should be in the form of a check or money order referencing your account number and this invoice number

RLNK11046254.

Once you receive your invoice for this order, you may pay your invoice by credit card. Please follow instructions provided at that time.

Make Payment To:

Copyright Clearance Center

Dept 001

P.O. Box 843006

Boston, MA 02284-3006

For suggestions or comments regarding this order, contact RightsLink Customer Support: customercare@copyright.com or +1-877-622-5543 (toll free in the US) or +1978-646-2777.

Gratis licenses (referencing $\$ 0$ in the Total field) are free. Please retain this printable license for your reference. No payment is required. 


\section{B.3 Chapter 4 Permission}

$11 / 23 / 11$

Help 'Assignment of copyright form - IOP Publishing Limiled'

\section{Assignment of copyright form - IOP Publishing Limited}

1. IOP Publishing Limited ("IOP") agrees to publlsh:

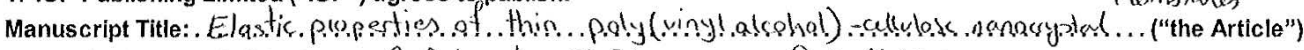

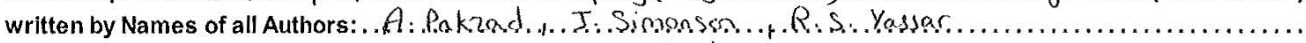

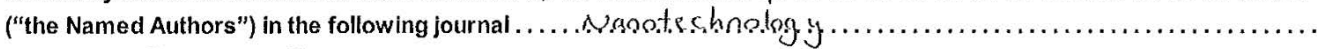
............ ("the Journal")

2. Transfer of Copyright Agreement

2.1 On acceptance for publication the undersigned author(s) ("Author") of the Article assigns exclusively to IOP worldwide copyright in the Aticle for the full term and for all media and formats in all material published as part of the Article, which expression includes but is not limited to the text, abstract, tables, figures, graphs, video abstracts and other multimedia content but excludes any other item referred to as supplementary material.

2.2 If any of the Named Authors are Government employees, on acceptance for publication the Author shall grant IOP a royalty free exclusive licence for the full term of copyright for all media and formats to do in relation to the Article all acts restricted by copyright worldwide.

2.3 On acceptance for publication the Author shall grant IOP a royalty free non-exclusive licence for the full term of copyright for all media and formats to do in relation to any supplementary material deemed to be part of the Article all acts restricted by copyright worldwide.

3. Author Rights

3.1 IOP grants the Named Authors the rights specified in 3.2 and 3.3. Al such rights must be exercised for non-commercial purposes, if possible should display citation information and IOP's copyright notice, and for electronic use best efforts must be made to include a link to the on-line abstract in the Journal. Exercise of the rights in 3.3 additionallymust not use the final published IOP format but the Named Author's own format (which may include amendments made following peer review).

3.2 The rights are:

3.2.1 To make copies of the Article (all or part) for teaching purposes;

3.2.2 To include the Articie (all or part) in a research thesis or dissertation;

3.2.3 To make oral presentation of the Article (all or part) and to include a summary and/or highlights of it in papers

distributed at such presentations or in conference proceedings; and

3.2.4 All proprietary rights other than copyright.

3.3 The additional rights are to:

3.3.1 Use the Article (all or part) without modification in personal compilations or publications of a Named Author's own works (provided not created by third party publisher);

3.3.2 Include the Article (all or part) on a Named Author's own personal web site;

3.3.3 Inciude the Article (all or part) on web sites of the Institution (including its repository) where a Named Author worked when research for the Article was carried out; and

3.3.4 No sooner than 12 months after publication to include the Article (all or part) on third party web sites including e-print servers, but not on other publisher's web sites.

\section{Signature}

In signing this Agreement the Author represents and warrants that the Article is the original work of the Named Authors, it has not been published previously in any form (other than as permitted under clause 3.2.2 which fact has been notified to IOP Publishing Ltd in writing), all Named Authors have participated sufficiently in the conception and writing of the Article, 
have received the final version of the Article, agree to its submission and take responsibility for it, and submission has been approved as necessary by the authorities at the establishment where the research was carried out.

The Author warrants that he/she signs this Agreement as authorised agent for all Named Authors and has the full power to enter into this Agreement and to make the grants it contains, that the Article has not been and will not be submitted to another publisher prior to withdrawal or rejection, it does not infringe any third party rights, it contains nothing libellous or unlawful, all factual statements are to the best of the Author's knowledge true or based on valid research conducted according to accepted norms, and all required permissions have been obtained in writing.

All Named Authors assert their moral rights.

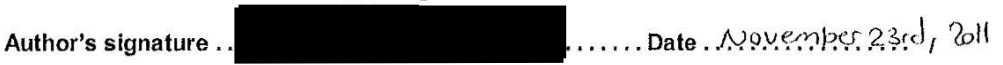

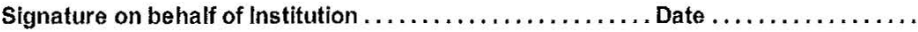

Note that if the Article was prepared as part of a Named Author's duties for their institution this Agreement must be signed by their institution as Author. Further, if there are several Named Authors it should be signed by one author in a representative capacity. If all Named Authors are employees of the US Government and the Article was prepared as part of their duties the Article is not eligible for copyright and one of the Named Authors should sign below to confirm this fact. If at least one of the Named Authors is not a US Government employee the above should be slgned.

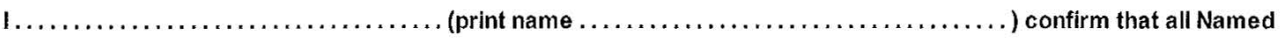
Authors are employees of the US Government and the Article was prepared as part of their dutles and consequently the Article is not eligible for copyright.

Please return AL $L$ of this form as soon as possible to IOP Publishing Ltd, Temple Circus, Temple Way, BRISTOL BS1 6BE, UK.

\section{Close this window}




\section{B.4 Chapter 5 Permission}

\section{RE: Permission request (Ref. No.: CSTE-D-11-}

00625R1)

From: M.Ravey@elsevier.com

To: apakzad@mtu.edu

Attachments: ole0.bmp (18.9KB)

\section{$\mathbf{x}$}

Dear Ms Pakzad,

We hereby grant you permission to reprint the material detailed below at no charge in your thes is subject to the following conditions:

1. If any part of the material to be used (for example, figures) has appeared in our publication with credit or acknowle dgement to another source, permission must also be sought from that source. If such permission is not obtained then that material may not be included in your publication/copies.

2. Suitable acknowledgment to the source must be made, either as a footnote or in a reference list at the end of your publication, as follows:

'This article was published in Publication title, Vol number, Author(s), Title of article, Page Nos, Copyright Elsevier (or appropriate Society name) (Year)."

3. Your thesis may be submitted to your in stitution in either print or electronic form.

4. Reproduction of this material is confined to the purpose for which permission is hereby given.

5. This permission is granted for non-exclusive world Englis h rights only. For other languages please reapply separately for each one required. Permission excludes use in an electronic form. Should you have a specific electronic project in mind please reapply for permission.

6. This includes permission for UMI to supply single copies, on demand, of the complete thesis. Should your thesis be published commercially, please reap ply for permission.

Kind regards

Michelle

Michelle Ravey :: Rights Associate:: Global Rights :: ELSEVIER

Currently working from home. Please contact me via:

E: m.ravey2@elsevier.com

From: Anahita Pakzad [mailto:apakzad@mtu.edu]

Sent: 17 November 2011 16:52

To: Rights and Permissions (ELS)

Cc: Reza Shahbazian Yassar

Subject: Permission request (Ref. No.: CSTE-D-11-00625R1) 
Dear Madam/Sir,

I am a PhD student at Michigan Technological University and I am including my article entitled "G adient of Nanomechanical Properties in the Interphase of Cellulose Nanocrystal Composites", that has been recently accepted for publication in Composites Science and Technology (please see bellow), as part of my PhD dissertation.

MTU dissertations are not used for commercial purposes. I would be very thankful if you provide me with the appropriate copyright permissions.

Thank you in advance for your time and consideration.

With best regards,

AP.

Amahita Pakad

PhD Candidate

Mechanical Engineening-Engine ering Mecharics

Michigan Technological University

Houghton, MI 4993 ?

..... Forw ardad Message ......

From: "Try-Wai Chos" <Chou Q edel.edus

To: reza $Q$ mts ade

Sent: Tuesday, November 15, 2011 11:24:36 AM GMT -05:00 US Canada Eastern

Subject: Your Submission

Ms. Ref. No: CSTE.D.11-00625R

Title: Gradient of Nancmechanical Properties in the Interghase of Cellulose Nanocrystal Composites

Composites Science and Technology

Dear Df. Yassar:

I am pleased to inform you that the above-referenced paper has been accepted for publication in Composites $\$$ cience and Technology

You will receive the galley proofs from Elsevier Science Ltd. in due course.

Thank you for choosing to publish your paper in Composites Science and Technology.

Sincerely yours,

Tsu-Wei Chov, PhD

Editor-in-Chief

Elsevier Limited. Registered Office: The Boulevard, Langford Lane, Kidlington, Oxford, OXS 1GB, United Kingdom, Registration No. 1982084 (England and Wales). 


\section{B.5 Chapter 6 Permission}

\section{E: Permission Request}

Monday, November 21, 2011 10:18:13 AM

From: LWolf@mrs.org

To: apakzad@mtu.edu

Dear Dr. Pakzad,

Because this is from your own work, our policy is that as an author of this article you have the right to use this material without our permission, remembering to cite the source. Please also keep this in mind for your future work that is published in the JMR .

\section{Lorraine}

JMR Editorial office

-----Original Message-----

From: Linda Baker

Sent: Monday, November 21, 2011 8:04 AM

To: Ellen Kracht; Lorraine Wolf

Subject: FW: Permission Request

This person apparently already contacted Cambridge, but since I have now knowledge of permissions or requests, can either one of you help him. -lb

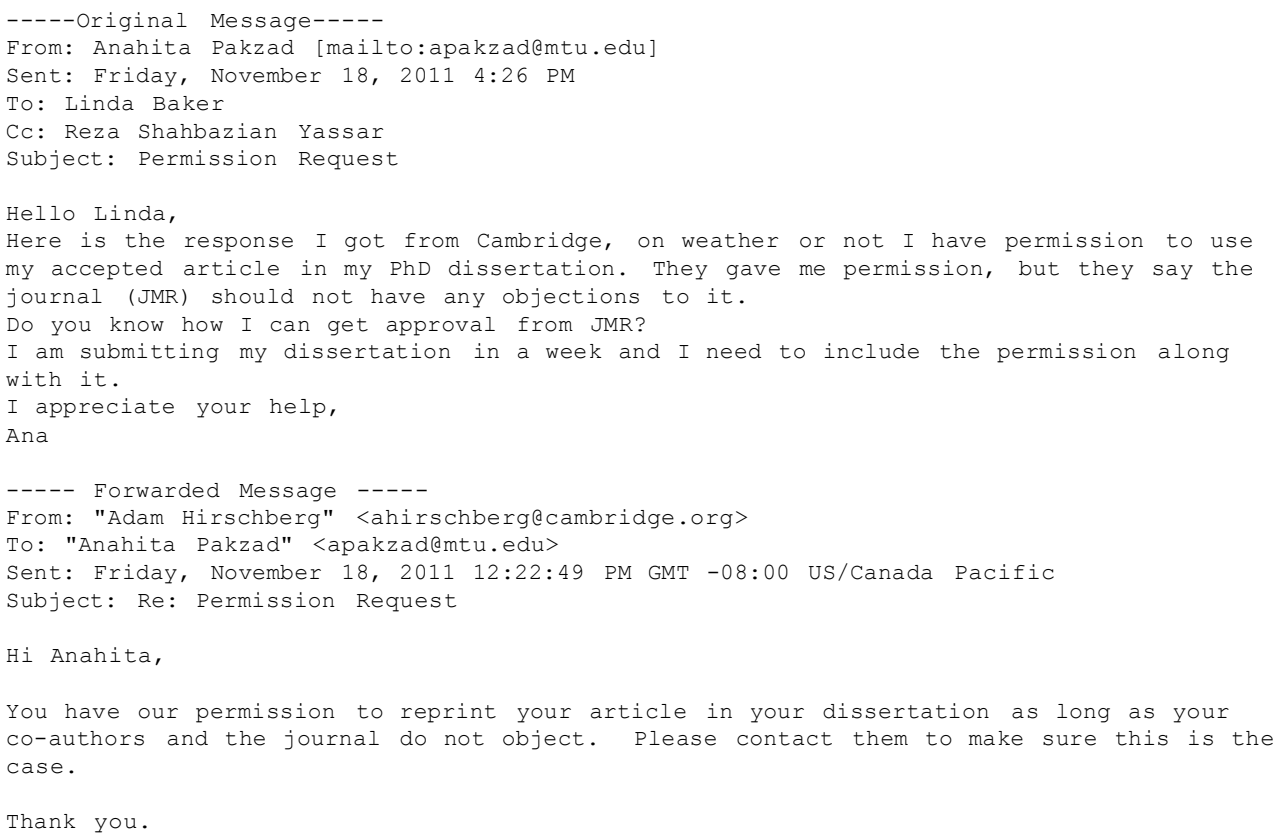




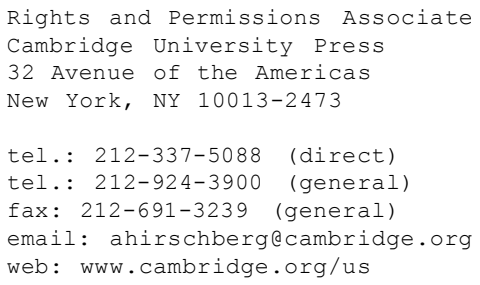

Yes, I am the first author.

Thanks for your help,

Anahita

----- Original Message -----

From: "Adam Hirschberg" <ahirschbergecambridge.org>

To: "Anahita Pakzad" <apakzademtu.edu>

Sent: Wednesday, November 16, 2011 12:02:21 PM GMT -08:00 US/Canada Pacific

Subject: Re: Permission Request

Are you one of the authors of "observation of size-scale effects on the nanomechanical properties of cellulose I nanocrystals?"

Adam

$\bar{A} \overline{d a m ~ H i r s c h b e r g}$

Rights and Permissions Associate

Cambridge University Press

32 Avenue of the Americas

New York, NY 10013-2473

tel.: 212-337-5088 (direct)

tel.: 212-924-3900 (general)

fax: 212-691-3239 (general)

email: ahirschbergecambridge.org

web: www.cambridge.org/us

From: Anahita Pakzad <apakzademtu.edu>

To: Adam Hirschberg <ahirschberg@cambridge.org>

CC: Reza Shahbazian Yassar <reza@mtu.edu>

Date: 11/16/2011 02:58 PM

Subject: Re: Permission Request

Hello Adam,

Our manuscript has been accepted for publication in the Focus issue of Journal of

Materials Research (JMR), February 2012.

Please let me know if you need more information.

Kind regards,

Anahita 
----- Original Message -----

From: "Adam Hirschberg" <ahirschbergecambridge.org>

To: "Permissions request" <apakzademtu.edu>

Sent: Wednesday, November 16, 2011 10:34:30 AM GMT -08:00 US/Canada Pacific Subject: Re: Permission Request

Dear Anahita,

Which Cambridge journal are you interested in?

Adam

Adam Hirschberg

Rights and Permissions Associate

Cambridge University Press

32 Avenue of the Americas

New York, NY 10013-2473

tel.: 212-337-5088 (direct)

tel.: 212-924-3900 (general)

fax: 212-691-3239 (general)

email: ahirschberg@cambridge.org

web: www.cambridge.org/us

From: Permissions request <apakzademtu.edu>

To: Web Email <ahirschbergecambridge.org>

Date: 11/11/2011 11:20 AM

Subject: Permission Request

date $=$ November 11 th, 2011

name = Anahita Pakzad

address1 = Michigan Technological University

address $2=1400$ Townsend Dr

address $3=$

town $=$ Houghton

postcode $=$ MI

country = USA

email = apakzademtu.edu

$f a x=925-463-0204$

tel $=906-370-6263$

title = journal, observation of size-scale effects on the nanomechanical properties of cellulose I nanocrystals pubyear $=2012$ book = editor bookauthor = Katerina E.

Aifantis, Reza Shahbazian-Yassar, Seung Min Han isbn = journalvol = Focus Issue, Feb 2012 details = full article will be used as a chapter in my PhD dissertation (both electronic and print) request = reprint language = by = author authorname = Anahita Pakzad workingtitle $=$ PhD dissertation title: Nanomechanics of Cellulose Crystals and Cellulose-Based Polymer Composites publisher = market $=$ world other $=$ no commercial use format $=$ print extract $=20 \%$ printrun $=$ pubdate $=2012$ sellprice $=$ no commercial use

Anahita Pakzad

PhD Candidate

Mechanical Engineering-Engineering Mechanics Michigan Technological University

Houghton, MI 49931 


\section{B.6 Appendix A and Chapter 1 Permissions}

WG: Copyright permission request

Wednesday, October 26, 2011 9:19:35 AM

From: Permissions.Heidelberg@springer.com

To: apakzad@mtu.edu

Sender: Alice.Essenpreis@springer.com

Dear Mr. Pakzad,

Thank you for your e-mail.

With reference to your request to reprint in your thesis material on which Springer

Science and Business Media control the copyright, permission is granted, free of charge, for the use indicated in your enquiry. Licenses are for one-time use only with a maximum distribution equal to the number that you identified in the licensing process.

This License includes use in an electronic form, provided it is password protected or on the university's intranet, destined to microfilming by UMI and University repository. For any other electronic use, please contact Springer at (permissions.heidelberg@springer.com)

The material can only be used for the purpose of defending your thesis, and with a maximum of 100 extra copies in paper.

Although Springer holds copyright to the material and is entitled to negotiate on rights, this license is only valid, provided permission is also obtained from the (co) author (address is given with the article) and provided it concerns original material which does not carry references to other sources (if material in question appears with credit to another source, authorization from that source is required as well). Permission free of charge on this occasion does not prejudice any rights we might have to charge for reproduction of our copyrighted material in the future.

Springer Science + Business Media reserves all rights not specifically granted in the combination of (i) the license details provided by you and accepted in the course of this licensing transaction, (ii) these terms and conditions and (iii) CCC's Billing and Payment terms and conditions.

Please include the following copyright citation referencing the publication in which the material was originally published. Where wording is within brackets, please include verbatim.

"With kind permission from Springer Science+Business Media: <book title, chapter title, volume, year of publication, page, name(s) of author(s), figure number(s), and any original (first) copyright notice displayed with material>."

This license is personal to you and may not be sublicensed, assigned, or transferred by you to any other person without Springer Science + Business Media's written permission. For future journal permission requests you are able to acquire permission via RightsLink: 
Kind regards,

Ālice Essenpreis

Springer

Rights and Permissions

$\bar{T}$ iergartenstrasse 17 | 69121 Heidelberg GERMANY

FAX: +4962214878223

permissions.Heidelberg@springer.com

www.springer.com/rights

Von: Duine, Maaike, Springer SBM NL Im Auftrag von Permissions Europe/NL

Gesendet: Mittwoch, 26. Oktober 2011 15:02

An: Permissions Heidelberg, Springer DE

Betreff: FW: Copyright permission request

From: Anahita Pakzad [mailto:apakzad@mtu.edu]

Sent: Monday, October 17, 2011 3:05 AM

To: Permissions Europe/NL

Cc: Reza Shahbazian Yassar

Subject: Copyright permission request

Dear Madam/Sir,

I am a PhD student at Michigan Technological University and I am including works listed bellow as part of my PhD dissertation:

(1) A. Pakzad, R.S. Yassar, "Mechanics of Cellulose Nanocrystals and their Polymer Composites" in: New Frontiers of Nanocomposite Materials: Novel Principles and Techniques, ed. A. Oechsner and A. Shokuhfar, Springer LLC, 1 ${ }^{\text {st }}$ Edition, (2011) (doi: 10.1007/8611_2010_38) (ISSB: 978-3-642-14696-1)

(2) R.S. Yassar, A. Pakzad, K. Momeni, "Principals of Atomic Force Microscopy" in: Encyclopedia of Tribology, ed. J. Wang and Y.-W. Chung, Springer LLC, submitted for publication (2011)

MTU dissertations are not used for commercial purposes. I would be very thankful if you provide me with the appropriate copyright permissions.

Thank you in advance for your time and consideration.

With best regards,

AP.

$--$

Anahita Pakzad

PhD Candidate

Mechanical Engineering-Engineering Mechanics

Michigan Technological University 


\section{B.7 Permission for Figure 11.1}

Rightslink Printable License
ELSEVIER LICENSE
TERMS AND CONDITIONS

Dec 15, 2011

This is a License Agreement between Anahita Pakzad ("You") and Elsevier ("Elsevier") provided by Copyright Clearance Center ("CCC"). The license consists of your order details, the terms and conditions provided by Elsevier, and the payment terms and conditions.

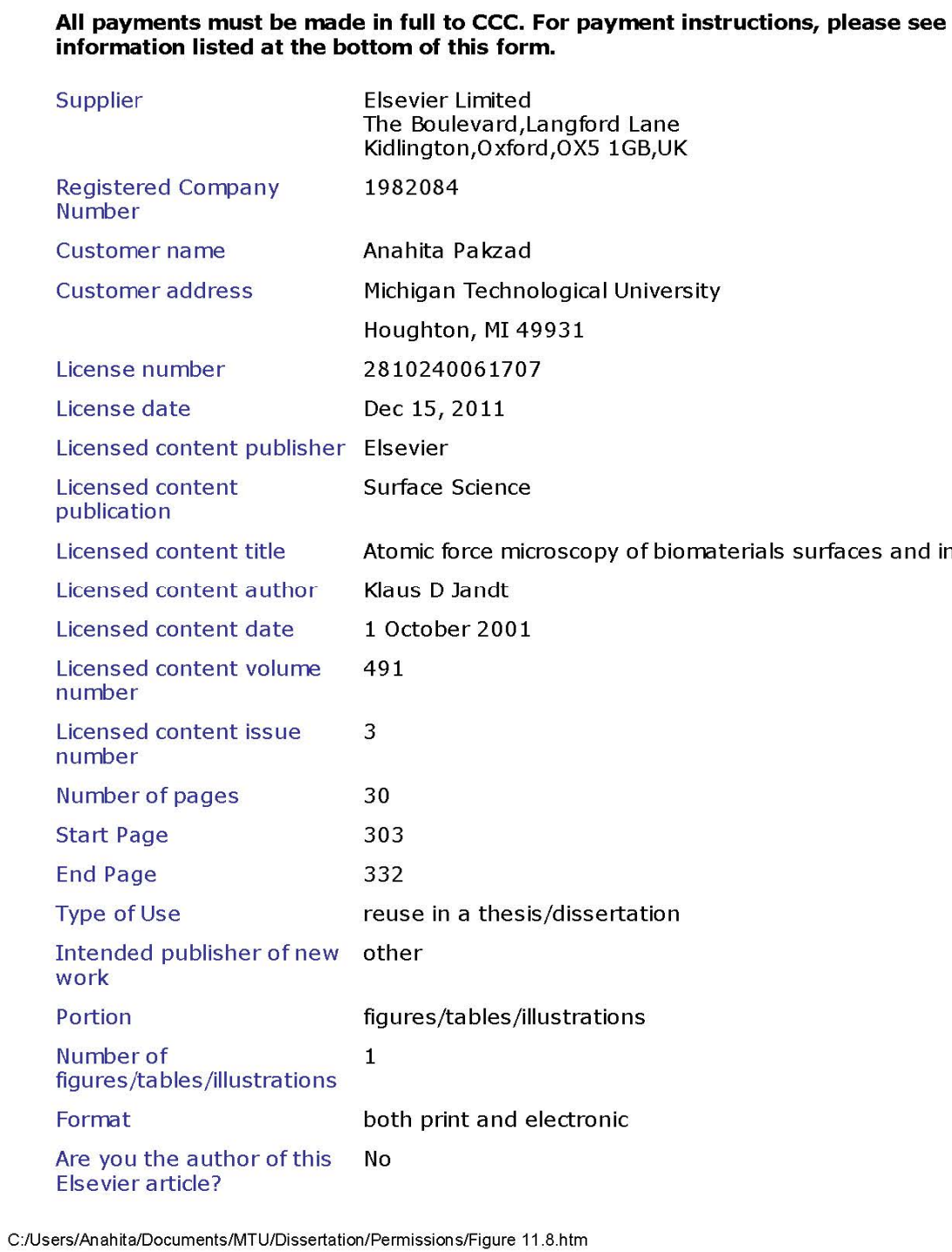




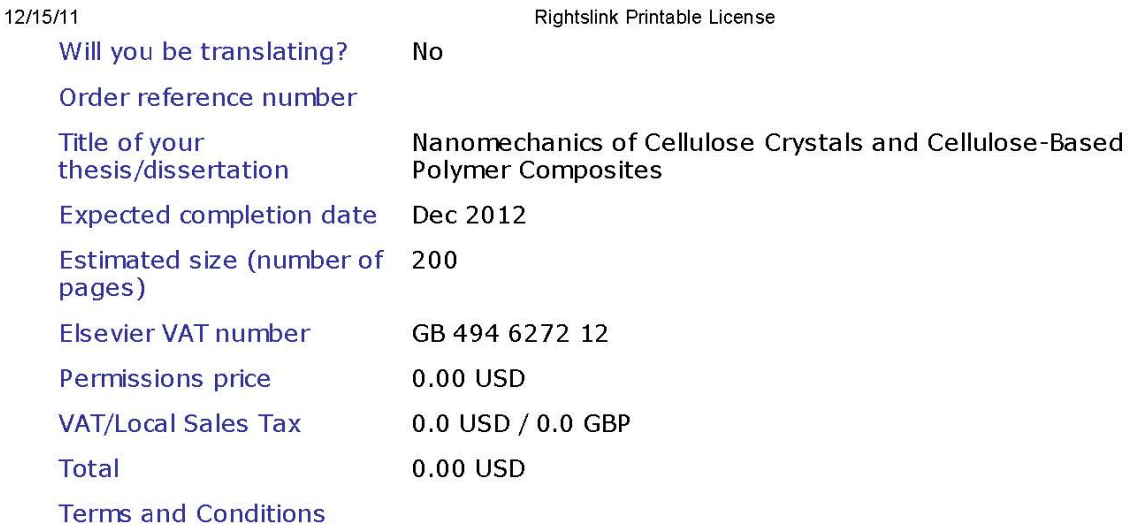

Terms and Conditions

\section{INTRODUCTION}

1. The publisher for this copyrighted material is Elsevier. By clicking "accept" in connection with completing this licensing transaction, you agree that the following terms and conditions apply to this transaction (along with the Billing and Payment terms and conditions established by Copyright Clearance Center, Inc. ("CCC"), at the time that you opened your Rightslink account and that are available at any time at http:/myaccount.copyright.com)

\section{GENERAL TERMS}

2. Elsevier hereby grants you permission to reproduce the aforementioned material subject to the terms and conditions indicated.

3. Acknowledgement: If any part of the material to be used (for example, figures) has appeared in our publication with credit or acknowledgement to another source, permission must also be sought from that source. If such permission is not obtained then that material may not be included in your publication/copies. Suitable acknowledgement to the source must be made, either as a footnote or in a reference list at the end of your publication, as follows:

"Reprinted from Publication title, Vol /edition number, Author(s), Title of article / title of chapter, Pages No., Copyright (Year), with permission from Elsevier [OR APPLICABLE SOCIETY COPYRIGHT OWNER]." Also Lancet special credit - "Reprinted from The Lancet, Vol. number, Author(s), Title of article, Pages No., Copyright (Year), with permission from Elsevier."

4. Reproduction of this material is confined to the purpose and/or media for which permission is hereby given.

5. Altering/Modifying Material: Not Permitted. However figures and illustrations may be altered/adapted minimally to serve your work. Any other abbreviations, additions, deletions and/or any other alterations shall be made only with prior written authorization of Elsevier Ltd. (Please contact Elsevier at permissions@elsevier.com)

6. If the permission fee for the requested use of our material is waived in this instance, please be advised that your future requests for Elsevier materials may attract a fee. 
7. Reservation of Rights: Publisher reserves all rights not specifically granted in the combination of (i) the license details provided by you and accepted in the course of this licensing transaction, (ii) these terms and conditions and (iii) CCC's Billing and Payment terms and conditions.

8. License Contingent Upon Payment: While you may exercise the rights licensed immediately upon issuance of the license at the end of the licensing process for the transaction, provided that you have disclosed complete and accurate details of your proposed use, no license is finally effective unless and until full payment is received from you (either by publisher or by CCC) as provided in CCC's Billing and Payment terms and conditions. If full payment is not received on a timely basis, then any license preliminarily granted shall be deemed automatically revoked and shall be void as if never granted. Further, in the event that you breach any of these terms and conditions or any of CCC's Billing and Payment terms and conditions, the license is automatically revoked and shall be void as if never granted. Use of materials as described in a revoked license, as well as any use of the materials beyond the scope of an unrevoked license, may constitute copyright infringement and publisher reserves the right to take any and all action to protect its copyright in the materials.

9. Warranties: Publisher makes no representations or warranties with respect to the licensed material.

10. Indemnity: You hereby indemnify and agree to hold harmless publisher and $\mathrm{CCC}$, and their respective officers, directors, employees and agents, from and against any and all claims arising out of your use of the licensed material other than as specifically authorized pursuant to this license.

11. No Transfer of License: This license is personal to you and may not be sublicensed, assigned, or transferred by you to any other person without publisher's written permission.

12. No Amendment Except in Writing: This license may not be amended except in a writing signed by both parties (or, in the case of publisher, by $\mathrm{CCC}$ on publisher's behalf).

13. Objection to Contrary Terms: Publisher hereby objects to any terms contained in any purchase order, acknowledgment, check endorsement or other writing prepared by you, which terms are inconsistent with these terms and conditions or CCC's Billing and Payment terms and conditions.

These terms and conditions, together with CCC's Billing and Payment terms and conditions (which are incorporated herein), comprise the entire agreement between you and publisher (and CCC) concerning this licensing transaction. In the event of any conflict between your obligations established by these terms and conditions and those established by CCC's Billing and Payment terms and conditions, these terms and conditions shall control.

14. Revocation: Elsevier or Copyright Clearance Center may deny the permissions described in this License at their sole discretion, for any reason or no reason, with a full refund payable to you. Notice of such denial will be made using the contact information provided by you. Failure to receive such notice will not alter or invalidate the denial. In no event will Elsevier or Copyright Clearance Center be responsible or liable for any costs, expenses or damage incurred by you as a result of a denial of your permission request, other than a refind of the amount(s) paid by you to Elsevier and/or Copyright Clearance Center for denied permissions.

\section{LIMITED LICENSE}


The following terms and conditions apply only to specific license types:

15. Translation: This permission is granted for non-exclusive world English rights only unless your license was granted for translation rights. If you licensed translation rights you may only translate this content into the languages you requested. A professional translator must perform all translations and reproduce the content word for word preserving the integrity of the article. If this license is to re-use 1 or 2 figures then permission is granted for non-exclusive world rights in all languages.

16. Website: The following terms and conditions apply to electronic reserve and author websites: Electronic reserve: If licensed material is to be posted to website, the web site is to be password-protected and made available only to bona fide students registered on a relevant course if:

This license was made in connection with a course,

This permission is granted for 1 year only. You may obtain a license for future website posting, All content posted to the web site must maintain the copyright information line on the bottom of each image,

A hyper-text must be inchided to the I Iomepage of the journal from which you are licensing at http:/www.sciencedirect.com/science/journal/xxxxx or the Elsevier homepage for books at http://www.elsevier.com , and

Central Storage: This license does not include permission for a scanned version of the material to be stored in a central repository such as that provided by Heron/XanEdu.

17. Author website for journals with the following additional clauses:

All content posted to the web site must maintain the copyright information line on the bottom of each image, and

he permission granted is limited to the personal version of your paper. You are not allowed to download and post the published electronic version of your article (whether PDF or HTML, proof or final version), nor may you scan the printed edition to create an electronic version,

A hyper-text must be inchuded to the Homepage of the journal from which you are licensing at http://www.sciencedirect.com/science/journal/xxxxx , As part of our normal production process, you will receive an e-mail notice when your article appears on Elsevier's online service ScienceDirect (www.sciencedirect.com). That e-mail will include the article's Digital Object Identifier (DOI). This number provides the electronic link to the published article and should be included in the posting of your personal version. We ask that you wait until you receive this e-mail and have the DOI to do any posting.

Central Storage: This license does not include permission for a scanned version of the material to be stored in a central repository such as that provided by Heron/XanEdu.

18. Author website for books with the following additional clauses:

Authors are permitted to place a brief summary of their work online only.

A hyper-text must be included to the Elsevier homepage at http://www.elsevier.com

All content posted to the web site must maintain the copyright information line on the bottom of each image

You are not allowed to download and post the published electronic version of your chapter, nor

C:/Users/Anahita/Documents/MTU/Dissertation/Permissions/Figure 11.8.htm 
may you scan the printed edition to create an electronic version.

Central Storage: This license does not include permission for a scanned version of the material to be stored in a central repository such as that provided by Heron/XanEdu.

19. Website (regular and for author): A hyper-text must be included to the Homepage of the journal from which you are licensing at $\mathrm{http} / / \mathrm{www}$.sciencedirect.com $/ \mathrm{science} / \mathrm{joumal} / \mathrm{xxxxx}$. or for books to the Elsevier homepage at http://www.elsevier.com

20. Thesis/Dissertation: If your license is for use in a thesis/dissertation your thesis may be submitted to your institution in either print or electronic form. Should your thesis be published commercially, please reapply for permission. These requirements include permission for the Library and Archives of Canada to supply single copies, on demand, of the complete thesis and include permission for UMI to supply single copies, on demand, of the complete thesis. Should your thesis be published commercially, please reapply for permission.

\section{Other Conditions:}

\section{v1.6}

If you would like to pay for this license now, please remit this license along with your payment made payable to "COPYRIGHT CLEARANCE CENTER" otherwise you will be invoiced within 48 hours of the license date. Payment should be in the form of a check or money order referencing your account number and this invoice number RLNK500684539. Once you receive your invoice for this order, you may pay your invoice by credit card.

Please follow instructions provided at that time.

Make Payment To:

Copyright Clearance Center

Dept 001

P.O. Box 843006

Boston, MA 02284-3006

For suggestions or comments regarding this order, contact RightsLink Customer Support: customercare@copyright.com or +1-877-622-5543 (toll free in the US) or +1-978-6462777.

Gratis licenses (referencing $\$ 0$ in the Total field) are free. Please retain this printable license for your reference. No payment is required. 